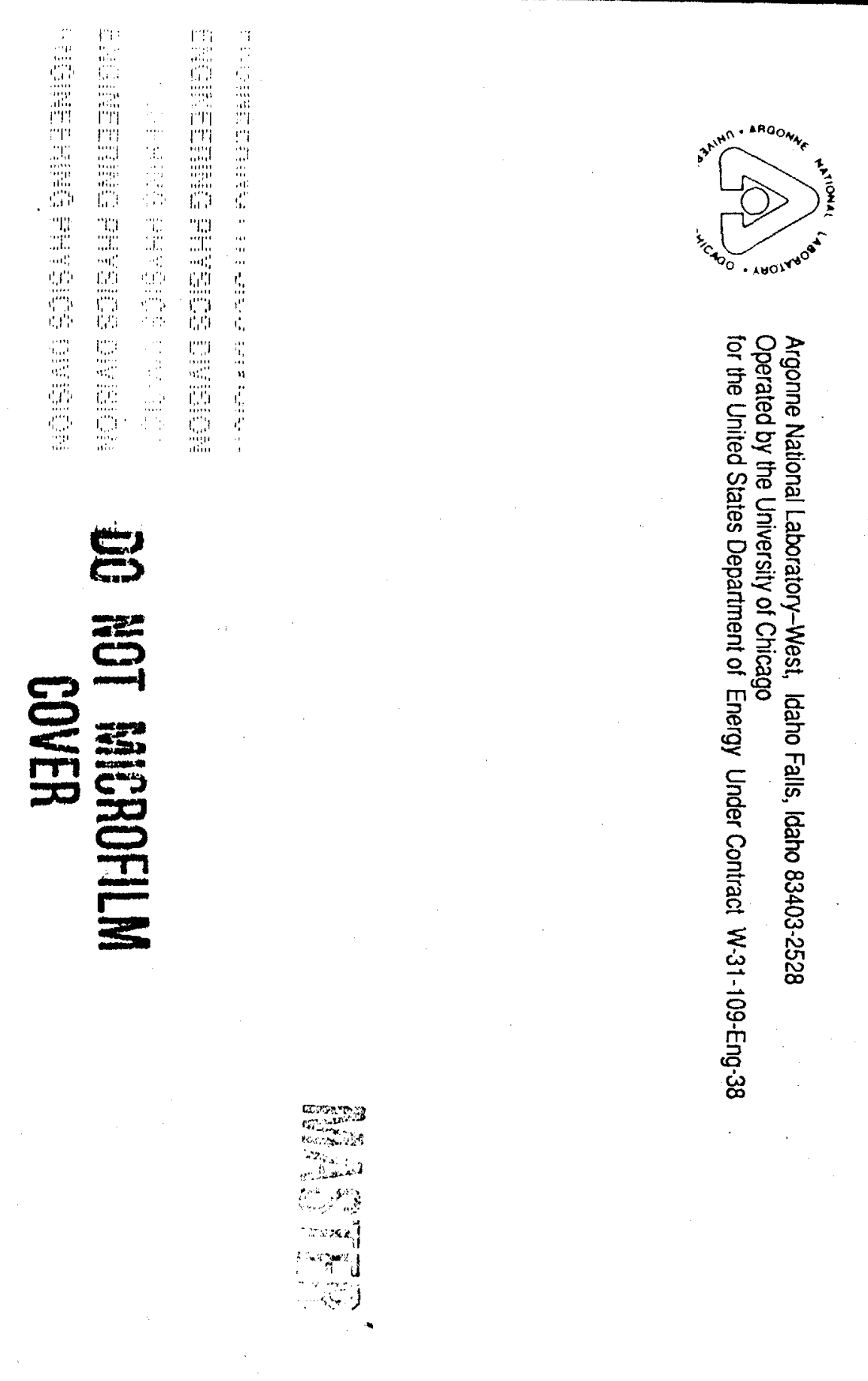

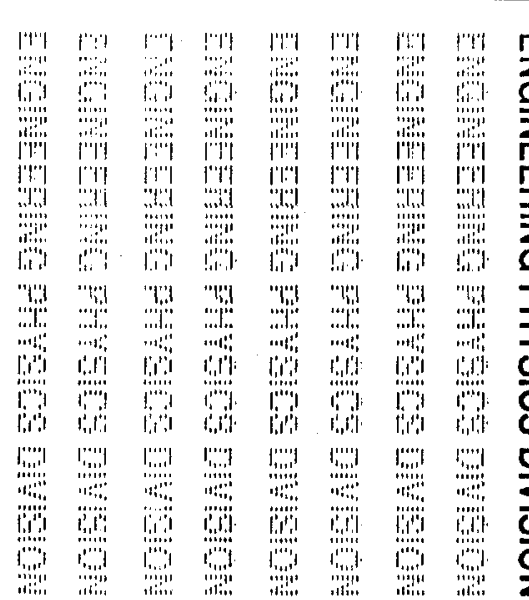

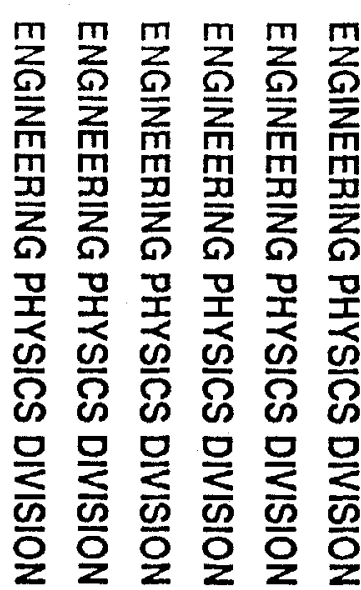

2
1
1
0
1
1
0

asti

紫

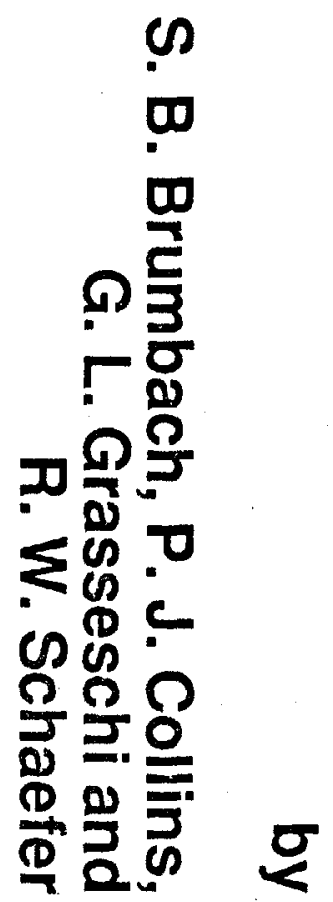

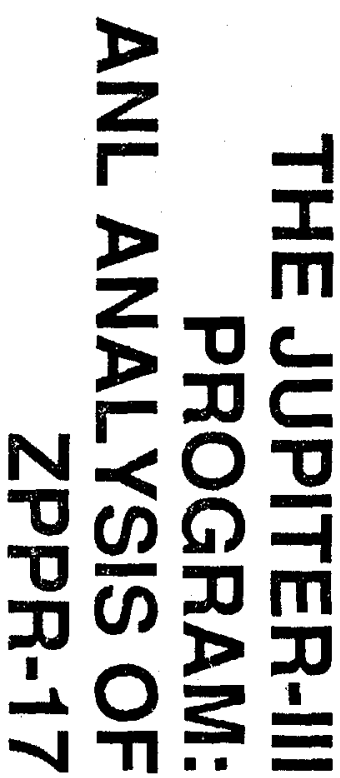

2
$⿱$
1
0
0
1
0
0 
Argonne National Laboratory, with facilities in the states of Illinois and Idaho, is owned by the United States government, and operated by The University of Chicago under the provisions of a contract with the Department of Energy.

\section{DISCLAIMER}

This report was prepared as an account of work sponsored by an agency of the United States Government. Neither the United States Government nor any agency thereof, nor any of their employees, makes any warranty, express or implied, or assumes any legal liability or responsibility for the accuracy, completeness, or usefulness of any information, apparatus, product, or process disclosed, or represents that its use would not infringe privately owned rights. Reference herein to any specific commercial product, process, or service by trade name, trademark manufacturer, or otherwise, does not necessarily constitute or imply its endorsement, recommendation, or favoring by the United States Government or any agency thereof. The views and opinions of authors expressed herein do not necessarily state or reflect those of the United States Government or any agency thereof. 


\title{
THE JUPITER-III PROGRAM: ANL ANALYSIS OF ZPPR-17
}

by

\author{
S. B. Brumbach, P. J. Collins, G. L. Grasseschi and R. W. Schaefer
}

\author{
Engineering Physics Division \\ Argonne National Laboratory - West \\ P.O. Box 2528 \\ Idaho Falls, ID 83403-2528
}

\begin{abstract}
DISCLAIMER
This report was prepared as an account of work sponsored by an agency of the United States Government. Neither the United States Government nor any agency thereof, nor any of their employees, makes any warranty, express or implied, or assumes any legal liability or responsibility for the accuracy, completeness, or usefulness of any information, apparatus, product, or process disclosed, or represents that its use would not infringe privately owned rights. Reference herein to any specific commercial product, process, or service by trade name, trademark, manufacturer, or otherwise does not necessarily constitute or imply its endorsement, recommendation, or favoring by the United States Government or any agency thereof. The views and opinions of authors expressed herein do not necessarily state or reflect those of the United States Government or any agency thereof.
\end{abstract}




\title{
THE JUPITER-III PROGRAM: ANL ANALYSIS OF ZPPR-17
}

by

S. B. Brumbach, P. J. Collins, G. L. Grasseschi and R. W. Schaefer

\begin{abstract}
The ZPPR-17 assembly was part of the JUPITER-III cooperative program between the U.S. DOE and PNC of Japan. The assembly was designed to study the neutronic behavior of a large, axially heterogeneous, liquid-metal-cooled reactor. The unique feature of the assembly was an internal blanket region in the axial center of the core extending two-thirds of the core radius. Assembly variants with 25 control rod positions and with 13 halfinserted control rods were built. This report describes in detail the results of measurements and analyses of ZPPR-17. The measurements emphasized reaction rate distributions, and gamma dose measurements were included. Additional measurements were control worth, sodium void worth, and reactivity coefficients associated with small material motions in assembly expansion and bowing.
\end{abstract}




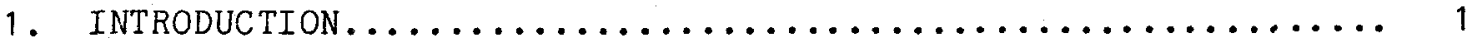

2. DESCRIPTION OF THE ASSEMBLIES................... 4

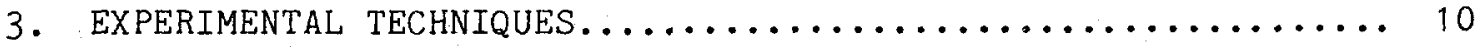

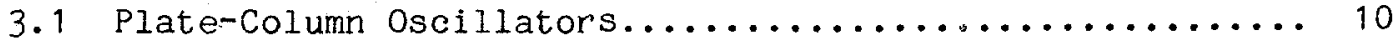

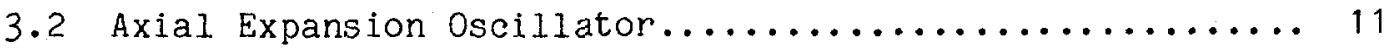

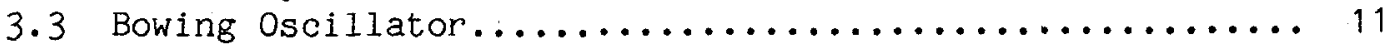

3.4 Gamma Ray Dose Measurements.................... 12

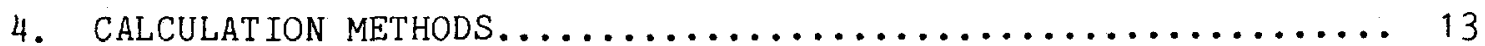

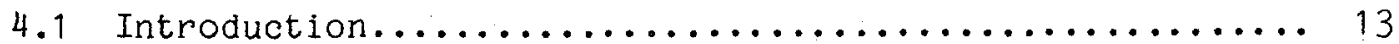

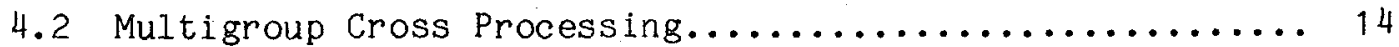

4.3 Improved Cross Sections for ZPPR $17 \ldots \ldots \ldots \ldots \ldots \ldots \ldots \ldots \ldots$

4.4 Reactor Models............................... 19

5. Calculated Eigenvalues and EFFEctive DELAyed........... 48 NEUTRON FRACTIONS

5.1 Experimental Reactivities and Nodal Diffusion Results.... 48

5.2 Results for Other Calculation Models............... 49

5.3 Summary of Eigenvalue Analysis................. 50

5.4 Delayed Neutron Fractions.................... 50

6. ANALYSIS OF REACTION RATE MEASUREMENTS ................ 61

6.1 Description of the Measurements................. 61

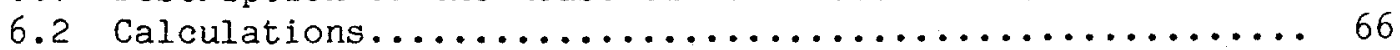

6.3 Reaction Rate Distributions in $2 \mathrm{PPR}-17 \mathrm{~A} \ldots \ldots \ldots \ldots \ldots \ldots 68$

6.4 Reaction Rate Distributions in $\mathrm{ZPPR}-17 \mathrm{~B} \ldots \ldots \ldots \ldots \ldots \ldots . \ldots 79$

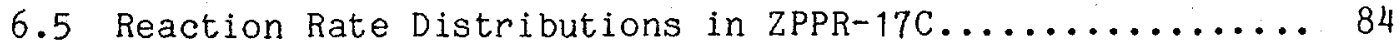

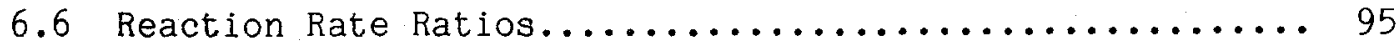

6.7 Comparison of Fission Rate Analys is in

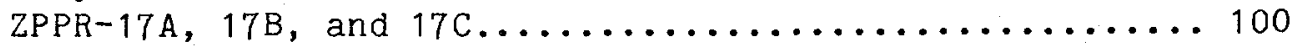

7. ANALYSis OF GaMma hEATING MEASUREMENTS In ZPPR -17 A........ 107

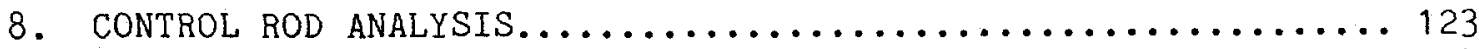

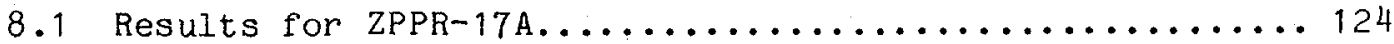

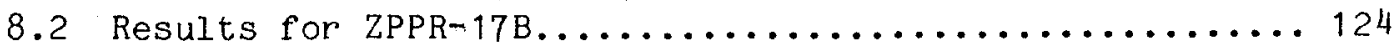

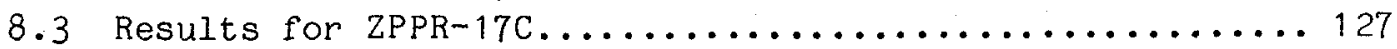

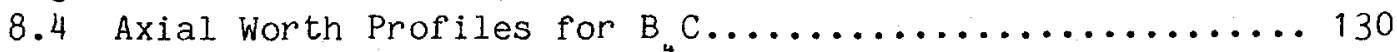

8.5 Additional Calculations for $\mathrm{ZPPR}-17 \mathrm{~B} \ldots \ldots \ldots \ldots \ldots \ldots \ldots \ldots \ldots \ldots$

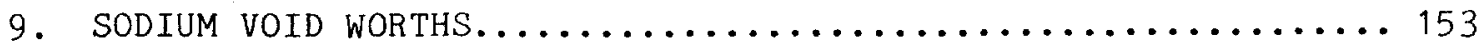

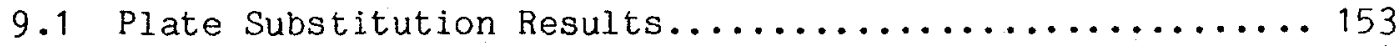

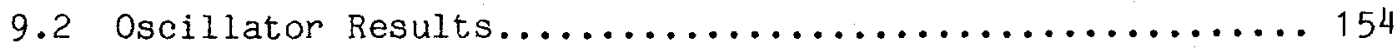




\section{DISCLAIMER}

Portions of this document may be illegible in electronic image products. Images are produced from the best available original document. 
10. CALCULATIONS OF BOWING, EXPANSION AND SMALL-SAMPLE WORTH..... 160 EXPERIMENTS IN ZPPR $-17 \mathrm{~A}$

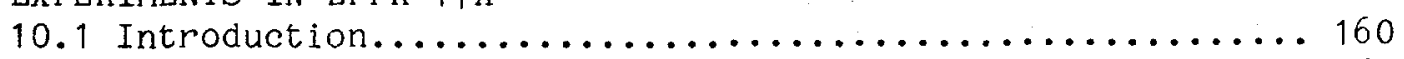

10.2 RTO and ATO Smali-Sample Worth Experiments........... 161

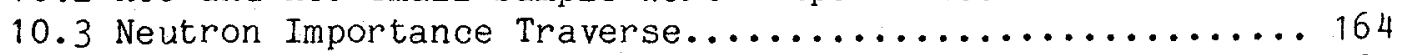

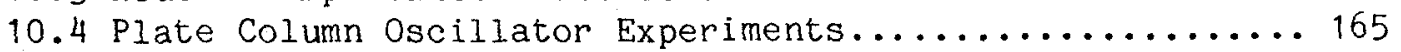

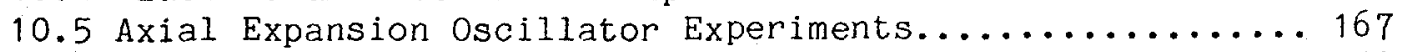

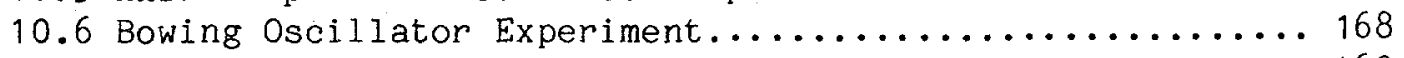

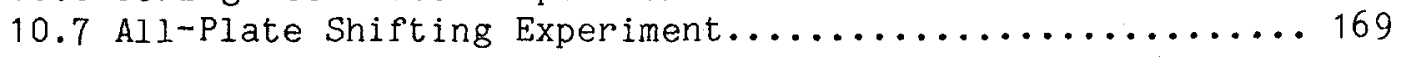

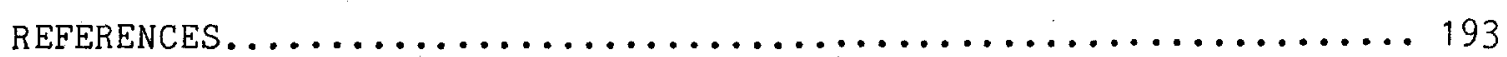




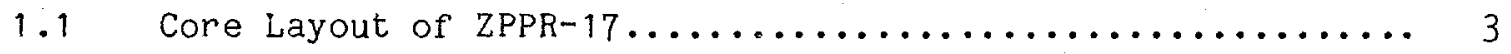

2. 1 Loading Pattern for Single-Fuel-Column Drawers........... 6 without Internal Blanket

2.2 Loading Pattern for Double-Fuel-Column Drawers........... 7 without Internal Blanket

2.3 Loading Pattern for Single-Fuel-Column Drawers.......... 8 with Internal Blanket Segment in ZPPR 17

2.4 Loading Pattern for Double-Fuel-Column Drawers........... 9 with Internal Blanket Segment in ZPPR-17

4.1 Slab Model used to Collapse Cross Sections for ZPPR-17A.... 43

4.2 The xyz Calculation Model for ZPPR 17 A................ 44

4.3 The xyz Calculational Model for ZPPR-17B............. 45

4.4 The xyz Calculational Model for ZPPR-17C (half one)...... 46

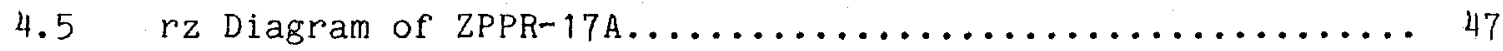

6.1 Foil Locations in $2 P P R-17 A$ in the $x y$ Plane........... 63

6,2 Foil Locations in ZPPR-17A in the $x z$ Plane............ 64

6.3 Foil Locations in $\mathrm{ZPPR}-17 \mathrm{~B}$ and $17 \mathrm{C}$ in the $\mathrm{xy}$ Plane.......65

6.4 Radial ${ }^{239} \mathrm{Pu}$ Fission Distribution at $5 \mathrm{~cm}$ from $\ldots \ldots \ldots \ldots 75$ the Midplane

6.5 Radial ${ }^{238} \mathrm{U}$ Fission Distribution at $5 \mathrm{~cm}$ from........... 76 the Midplane

6.6 Radial ${ }^{239} \mathrm{Pu}$ from Distribution at $18 \mathrm{~cm}$ from........... 77 the Midplane

6.7 Radial ${ }^{238} \mathrm{U}$ Fission Distribution at $18 \mathrm{~cm}$ from.......... 78 the Midplane

6.8 Measured Axial Distributions for Fission in ${ }^{23} \mathrm{~s} . \ldots . \ldots . . .92$ Adjacent to the Central Control Position in ZPPR-17B and in $\mathrm{ZPPR}-17 \mathrm{C}$

6.9 Measured Axial Distributions for Fission in ${ }^{235} \mathrm{U}$ Adjacent... 93 to an Outer Control Position in ZPPR -17B and in ZPPR-17C 
LIST OF FIGURES (contd)

Page

6.10 Measured ${ }^{235} \mathrm{U}$ Eission Distributions at Six Axial Heights.... 94 along the $x$-axis in ZPPR-17C

7.1 RZ Plot of $\mathrm{ZPPR}-17$ and TLD Locations................. 119

7.2 Measured and Calculated Doses in a Double-Fuel-Column Cell.. 120

7.3 Measured and Calculated Total Dose Rates along the x-axis... 121 of $\mathrm{ZPPR}-17 \mathrm{~A}$

7.4 Measured and Calculated Axial Distributions of Total...... 122 Dose Rates in ZPPR-17A

8.1 Control Positions used in ZPPR-17A Measurements.......... 147

8.2 Loading Pattern for Close-packed Pin Control Rod.......... 148

8.3 Worth Profile for Mockup Central Control Rod in ZPPR-17B.... 149

8.4 Comparison of Axial Worth Profiles of $B_{4} C$ in $2 P P R-17 B \ldots \ldots 150$

8.5 Measured Axial Worth Profile for $\mathrm{B}_{4} \mathrm{C}$ in $149-49 / 249-49 \ldots \ldots 151$ in $\mathrm{ZPPR}-17 \mathrm{C}$

8.6 Measured Axial Worth for $\mathrm{B}_{4} \mathrm{C}$ in $165-49 / 265-49$ in ZPPR-17C... 152

9.1 Locations of Sodium Void Worth Measurements in ZPPR-17A.... 157

9.2 Reactivity versus Sodium Column Displacement for......... 158 Various Locations in ZPPR-17A

9.3 Calculated and Measured Sodium Void Worths as a Function.... 159 of Axial Position in ZPPR-17A

10.1 Locations of Plate Shifting Zone and Oscillators......... 186 in $\mathrm{ZPPR}-17 \mathrm{~A}$

10.2 Radial Traverse for PU-30 Sample in ZPPR-17A......... 187

10.3 Radial Traverse for DU-6 Sample in ZPPR-17A........... 188

10.4 First Axial Traverse (ATO-1) for PU-30 Sample in ZPPR-17A... 189

10.5 First Axial Traverse (ATO-1) for B-1 Sample in ZPPR-17A.... 190

10.6 First Axial Traverse (ATO-1) for U-6 Sample in ZPPR-17A.... 191

10.7 Neutron Importance Radial Traverse in ZPPR $17 \mathrm{~A} \ldots \ldots \ldots \ldots . \ldots 192$ 
2.1 Characteristics of the $2 P P R-17$ Reference $\ldots \ldots \ldots \ldots \ldots \ldots \ldots$ Critical Configurations

4.1 Energy Structure of the Cross Section Sets used for....... 21 ZPPR-17A Analysis

4.2 Effect of New Cross Sections on ZPPR-17A Eigenvalue....... 22

4.3 Axial Reaction Rate Traverses with 0ld and New........... 23 Cross Sections

4.4 Sensitivity of ${ }^{239} \mathrm{Pu}$ Fission in the Inner Blanket........ 24 to Cross Section Type

4.5 ZPPR Assembly 17A: Atom Densities by Zone............. 25

4.6 ZPPR Assembly 17B: Atom Densities by Zone............ 30

4.7 ZPPR Assembly 17C: Atom Densities by Zone............ 35

5.1 Experimental and Calculated k-effective $\ldots \ldots \ldots \ldots \ldots \ldots \ldots 2$ Results for ZPPR-17A

5.2 Experimental and Calculated k-effective.............. 53 Results for ZPPR-17B

5.3 Experimental and Calculated k-effective.............. 54 Results for ZPPR-17C

5.4 k-effective Calculations for Several Models of ZPPR-17A.... 55

5.5 k-effective Calculations for Several Models of ZPPR-17B.... 56

5.6 k-effective Calculations for Several Models of ZPPR-17C.... 57

5.7 Modelling Effects Calculated for ZPPR-17A........... 58

5.8 ZPPR -17 Fuel Loadings and Criticality Predictions......... 59

5.9 Delayed Neutron Parameters for ZPPR-17A............. 60

5.10 Delayed Neutron Parameters for ZPPR-17B............. 60

5.11 Delayed Neutron Parameters for ZPPR-17C............. 60

6.1 ZPPR-17A: Radial Reaction Rate Distributions along....... 70 the $X$-axis at $z=5 \mathrm{~cm}$ 
6.2 ZPPR-17A: Summary of Radial Reaction Rate Distribution.... 71 C/E Values

6.3 ZPPR-17A: Axial Reaction Rate Distributions in......... 72 Matrix 148-50 (Core Center)

6.4 ZPPR-17A: Axial Reaction Rate Distributions in.......... 73 Matrix 148-66 (Outer Core)

6.5 ZPPR-17A: Axial Reaction Rate Distributions in ........ 74 Matrix 148-70 (Outer Core)

6.6 ZPPR-17B: Summary of Radial Reaction Rate ........... 80 Distribution Analyses

6.7 ZPPR-17B: Axial Reaction Rate Distributions in ........ 81 Matrix 148-51 (Near Core Center)

6.8 ZPPR-17B: Axial Reaction Rate Distributions in......... 82 Matrix 138-62 (next to CRP)

6.9 ZPPR-17B: Axial Reaction Rate Distributions in......... 83 matrix 148-70 (Outer Core)

6.10 ZPPR-17C: Summary of Radial Reaction Rate............ 85 Distribution Analysis

6.11 ZPPR-17C: Axial Reaction Rate Distributions in......... 86 Matrix 148-51 (near Core Center)

6.12 ZPPR-17B: Axial Reaction Rate Distributions in......... 87 Matrix 248-51 (near Core Center)

6.13 ZPPR-17C: Axial Reaction Rate Distributions in......... 88 Matrix 138-62 (next to CRP)

6.14 ZPPR-17C: Axial Reaction Rate Distributions in......... 89 Matrix 238-62 (next to $C R$ )

6.15 ZPPR-17C: Axial Reaction Rate Distributions in..........990 Matrix 148-70 (Outer Core)

6.16 ZPPR-17C: Axial Reaction Rate Distributions in.......... 91 Matrix 248-70 (Outer Core)

6.17 ZPPR-17A: Summary of Reaction Rate Ratio Analysis........ 96

6.18 ZPPR-17B: Summary of Reaction Rate Ratio Analysis........ 97 
6.19 ZPPR 17C: Summary of Reaction Rate Ratio Analysis........ 98

6.20 Reaction Rate Ratio C/E Results for ZPPR-17A, 17B and 17C... 99

6.21 Comparison of Radial ${ }^{235} \mathrm{U}$ Fission Rate $\mathrm{C} / \mathrm{E}$ Values......... 102 in $\mathrm{ZPPR}-17 \mathrm{~A}, 17 \mathrm{~B}$, and $17 \mathrm{C}$

6.22 Comparison of $\mathrm{C} / \mathrm{E}$ Values for ${ }^{2{ }^{3 y} \mathrm{Pu}}$ Fission Axial ......... 103 Traverses Near the Center of ZPPR-17A, 17B, and 17C

6.23 Comparison of $\mathrm{C} / \mathrm{E}$ Values for ${ }^{23 \mathrm{~s}} \mathrm{U}$ Fission Axial .......... 104 Traverses Near the Center of ZPPR $17 \mathrm{~A}, 17 \mathrm{~B}$, and $17 \mathrm{C}$

6.24 Comparison of $\mathrm{C} / \mathrm{E}$ Values for ${ }^{235} \mathrm{U}$ Fission Axial ......... 105 Traverses Near the Edge of the Inner Blanket in ZPPR-17A, $17 \mathrm{~B}$, and $17 \mathrm{C}$

6.25 Comparison of $\mathrm{C} / \mathrm{E}$ Values for ${ }^{235} \mathrm{U}$ Fission Axial.......... 106 Traverses in 1,248-70 (Outer Core) in ZPPR-17A, $17 \mathrm{~B}$, and $17 \mathrm{C}$

7.1 Summary of Cell Averaging Factors.................. 110

7.2 Broad Group Structures for Neutron and Gamma Cross....... 111 Section Library

7.3 Neutron and Gamma Response Functions for TLDs.......... 112

7.4 Mass Absorption Coefficients for Stainless Steel......... 113

7.5 Measured and Calculated Dose Rates along the $\mathrm{X}$-axis....... 114 at 3 in. from the Midplane

7.6 Measured and Calculated Dose Rates along the X-axis....... 115 at 9 in. from the Midplane

7.7 Measured and Calculated Dose Rates in the Axial......... 116 Direction in Matrix 149-53

7.8 Measured and Calculated Dose Rates in the Axial......... 117 Direction in Matrix 149-60

7.9 Measured and Calculated Dose Rates in the Axial......... 118 Direction in Matrix 149-70

8.1 Recommended Control and CRP worths in ZPPR-17A and...... 133 Comparison with Calculation

8.2 Worths of Control Rod Banks in $Z^{2}$ PPR-17B............. 134 
8.3 Worths of Rod Bank Combinations in ZPPR-17B........... 135

8.4 Worths of Rod Banks with Missing Rods in ZPPR $-17 B \ldots \ldots \ldots \ldots 136$

8.5 Control Rod Interaction Effects in ZPPR $-17 B \ldots \ldots \ldots \ldots \ldots \ldots 137$

8.6 Worth of Single Rods Withdrawn from Rod Banks in ZPPR-17B... 138

8.7 Calculated Control Rod worths in ZPPR-17B using Nodal...... 139 Diffusion and Nodal Transport Models

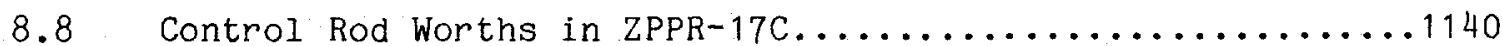

8.9 Control Rod Interaction Effects in ZPPR $-17 \mathrm{C} \ldots \ldots \ldots \ldots \ldots \ldots 141$

8.10 Comparison of Control Rod Worths in ZPPR -17C using....... 142 Different Calculation Models

8.11 Comparison of Control Rod Worths in ZPPR-17B and ZPPR-17C... 143

8.12 Calculations of Control Rod Worths in ZPPR-17B using...... 144 21 Group Data

8.13 Calculations of Control Rod worths in ZPPR-17B with....... 145 the TRITAC Code

8.14 Calculations of Control Rod Banks in ZPPR 17 B by ........ 146 Different Methods

9.1 Step Reactivity Worths for Sodium Voiding in ZPPR-17A..... 156

9.2 Sodium Void Results for Inner Core Zones in ZPPR-17A...... 157

10.1 Description of ZPPR-17A Oscillator Measurements......... 171

10.2 PU-30, RTO Worth Traverse......................... 172

10.3 DU -6 , RTO Worth Traverse....................... 173

10.4 PU-30, ATO-1 Worth Traverse...................... 174

10.5 B-1, ATO-1 Worth Traverse........................ 175

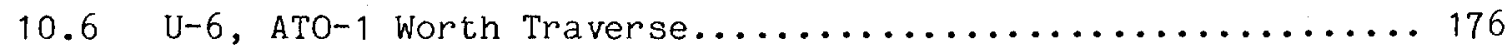

10.7 Difference Between RTO and ATO C/Es at Similar Positions.... 177

10.8 Region-Average C/Es from ZPPR-17A RTO and ATO Traverses.... 178 


\section{LIST OF TABLES (contd)}

Page

10.9 Neutron Importance Radial Traverse................. 179

10.10 Clad Pu U + SS Column Worth Traverse, PCO $3 \ldots \ldots \ldots \ldots \ldots \ldots$

$10.11 \mathrm{U}_{3} \mathrm{O}_{8}$ Column Worth Traverse, $\mathrm{PCO}-4 \ldots \ldots \ldots \ldots \ldots \ldots \ldots \ldots \ldots$

10.12 Comparison of C/Es from PCO vs. RTO and ATO Experiments.... 182

10.13 Representative Core Region C/Es from PCO Experiments....... 183

10.14 Axial Expansion Oscillator Results.................... 184

10.15 Bowing Oscillator C/E Adjustments from YZ Model......... 185 Calculations 


\section{INTRODUCTION}

ZPPR-17 was the first of two assemblies in the JUPITER-III Cooperative program between the US DOE and PNC of Japan. The ZPPR-17 assembly was designed to study the neutronic behavior of a large, axially heterogeneous, liquid-metal-cooled reactor (LMR). The ZPPR-17 composition represented mixed plutonium- and depleted uranium-oxide fuel with sodium coolant and stainless steel structural material. The unique feature of the assembly was the presence of an internal blanket region in the axial center of the core extending two-thirds of the core radius.

There were three phases in the ZPPR-17 program, each represented by an assembly configuration, designated $A, B$, and $C$. The phase A conf iguration was a physics benchmark containing neither mockup control rods (CRs) nor control rod positions (CRPS). Phase B contained $25 \mathrm{CRPs}$. In phase C, 13 of the CRPs contained half-inserted CRs. The core layout, including CRP locations, is given in Fig. 1.1 .

This report describes in detail the results of the ANL analyses of the three phases of ZPPR-17. The measurements were conducted between January and July, 1987. Assembly descriptions, measurement results, and many analysis results have been presented in the ZPPR Progress Report series. ${ }^{1-5}$ Other reports describe results from earlier JUPITER programs. JUPITER-I results for conventional cores are in references 6 and 7 and JUPITER-II results for radially heterogeneous cores are in reference 8 . This report was compiled primarily for discussions at the JUPITER Analysis Meeting to be held at ANL-West between September 13 and 15, 1988.

The experimental program was designed to emphasize those parameters most sensitive to the presence of the internal blanket. Consequently, power distributions were measured in unusual detail with numerous axial distributions and radial distributions at different axial heights. Detailed gamma dose distributions were also measured. As CRPs and later CRs were added, their effects on power distribution were measured by repeating the foil and TLD irradiations made in ZPPR $17 \mathrm{~A}$. 
Control rod worths were also an important part of the program. The control rod worth measurements in ZPPR-17A were a series of studies of rods of varying size and boron enrichment and the worth of CRP banks. In 17B, 20 control rod measurements were made including rod banks, combinations of rod banks and banks with missing rods. In $17 \mathrm{C}$, the worths of secondary control rods were measured relative to a core with the primary rods half inserted. Measurements of axial worth profiles of $\mathrm{B}_{4} \mathrm{C}$ rods were made in $17 \mathrm{~B}$ and $17 \mathrm{C}$.

Sodium void worths were measured at several radial locations, with emphasis on the axial worth profile for sodium. In addition to traditional zone voiding methods, a new device called the plate-column oscillator was used in the void-worth experiments.

Additional experiments discussed here concerned reactivity coefficients associated with small material motions in assembly expansion and bowing. New experimental methods and devices were employed.

Calculations were made with ENDF/B-V.2 cross section data. Previous JUPITER analysis has been with ENDE/B-IV. Cross sections were processed for cell heterogeneity with both the commonly used "asymptotic cell" models and with a "buckling recycle" technique. Most reactor calculations were made with xyz-geometry models in either 21 or 6 energy groups with diffusion theory. A number of calculations were also made with transport methods in xyz geometry.

The fundamental to first (azimuthal) eigenvalue separation for $2 P P R-17 B$ was calculated to be $4.0 \% \Delta k$. This is somewhat less than the $5.3 \%$ for the similar-size conventional core $2 P P R-9$, but greater than the $2.7 \%$ for the radially heterogeneous $Z P P R-13 A$. Although $Z P P R-17$ was an axially heterogeneous core, the eigenvalue separation for the first axial harmonic was about $20 \% \Delta k$. Consistent with these modest eigenvalue separations, kinetics experiments in ZPPR-17 showed only minor decoupling effects similar to those seen earlier in ZPPR-9. 


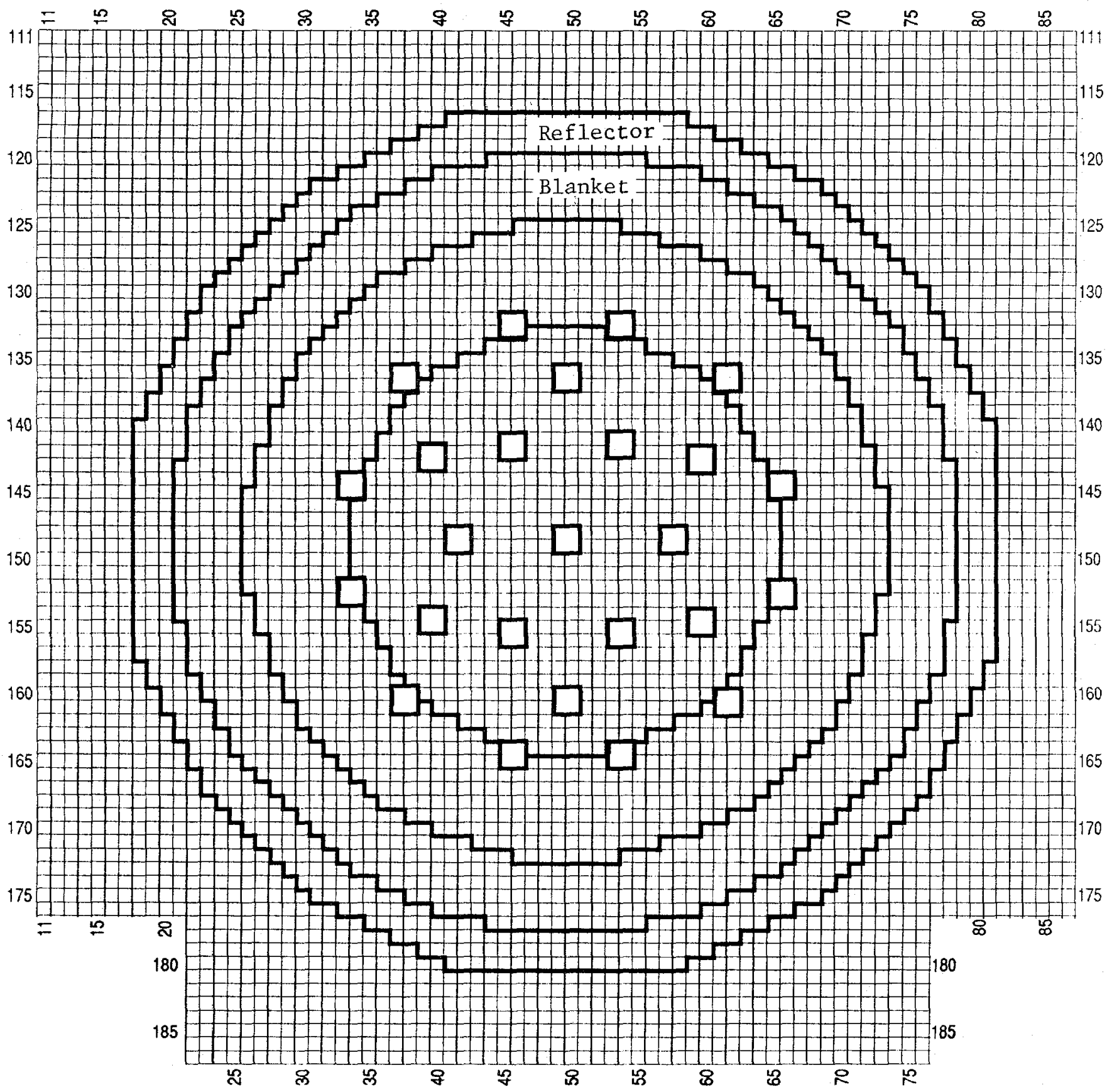

Fig. 1.1 Core Layout of ZPPR-17. 


\section{DESCRIPTION OF THE ASSEMBLIES}

The ZPPR-17 assemblies had distinct regions of internal blanket, core, outer radial blanket, axial blanket and radial and axial reflectors. There were two basic types of core cells; those containing either one or two columns of fuel. Reactivity was adjusted by changing the ratio of singleto double-fuel-column drawers. Both core cell types had versions with and without internal blanket regions. The internal blanket occupied the first $152 \mathrm{~mm}$ of the drawer. The radial extent of the internal blanket was about $870 \mathrm{~mm}$. For convenience, that radial portion of the core containing internal blanket is called "inner core" and that portion without internal blanket is called "outer core." The ratio of single to double-fuel-column drawers was essentially constant throughout the core, simulating a single fuel enrichment.

The pattern of single- and double-fuel-column drawers was essentially the same in the inner and outer core regions resulting in a uniform fuel enrichment throughout the core. The single-fuel-column cell without internal blanket is shown in Fig. 2.1 and the double-fuel-column cell is shown in Fig. 2.2. The corresponding cells with internal blanket segments are shown in Figs. 2.3 and 2.4 Only the standard ZPPR Pu-U-Mo alloy fuel was used. The core volume including internal blanket was 5518 liters. The volume of the internal blanket was 741 liters.

Detailed descriptions of $Z \mathrm{PPP}-17 \mathrm{~A}$ were reported in reference 1; $Z P P R-17 B$ in reference 2 and $Z P P R-17 C$ in reference 3 . A summary of the salient features of the three assembly variants is given in Table 2.1. 
TABLE 2.1 Characteristics of the ZPPR-17 Reference Critical Configurations

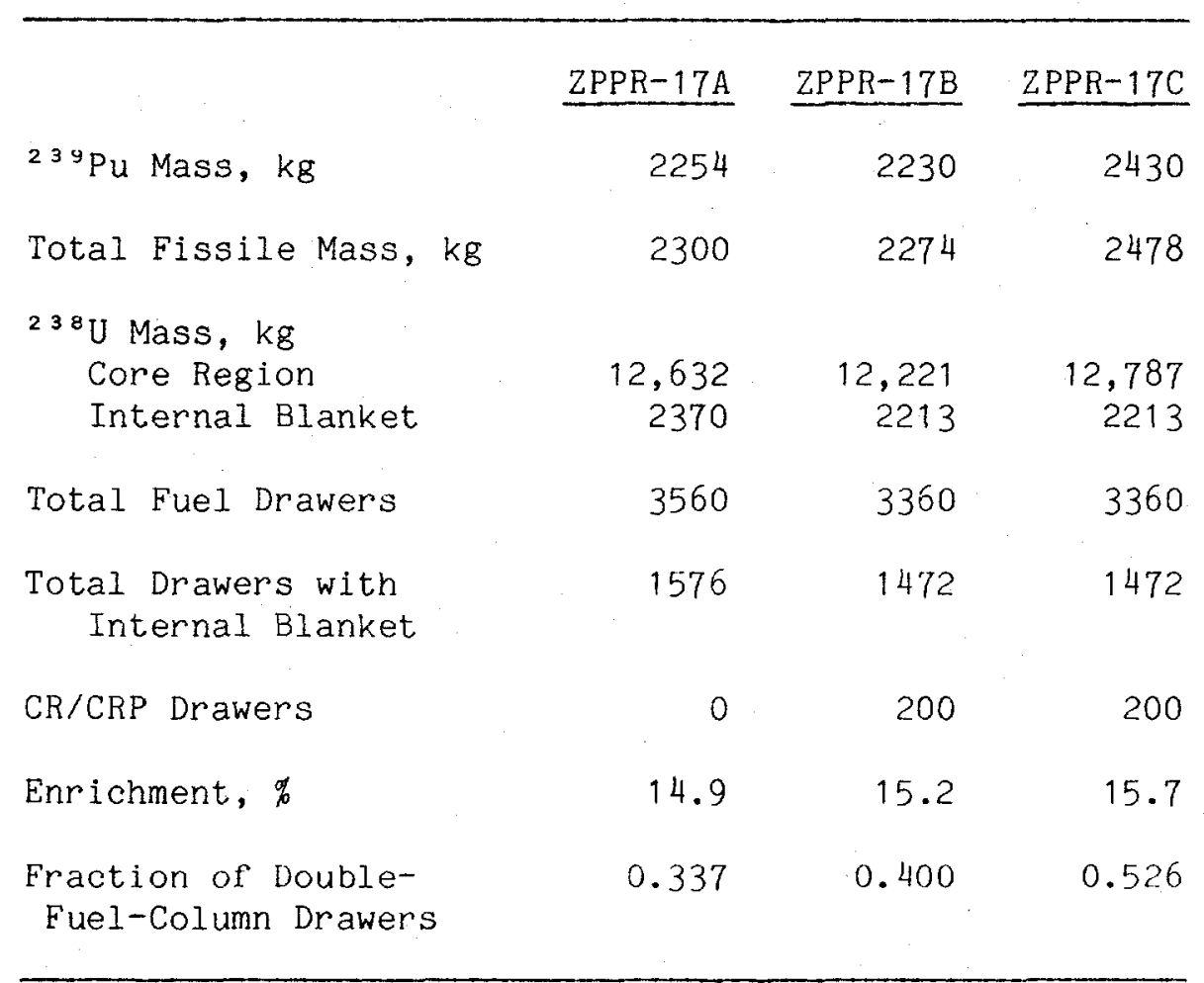




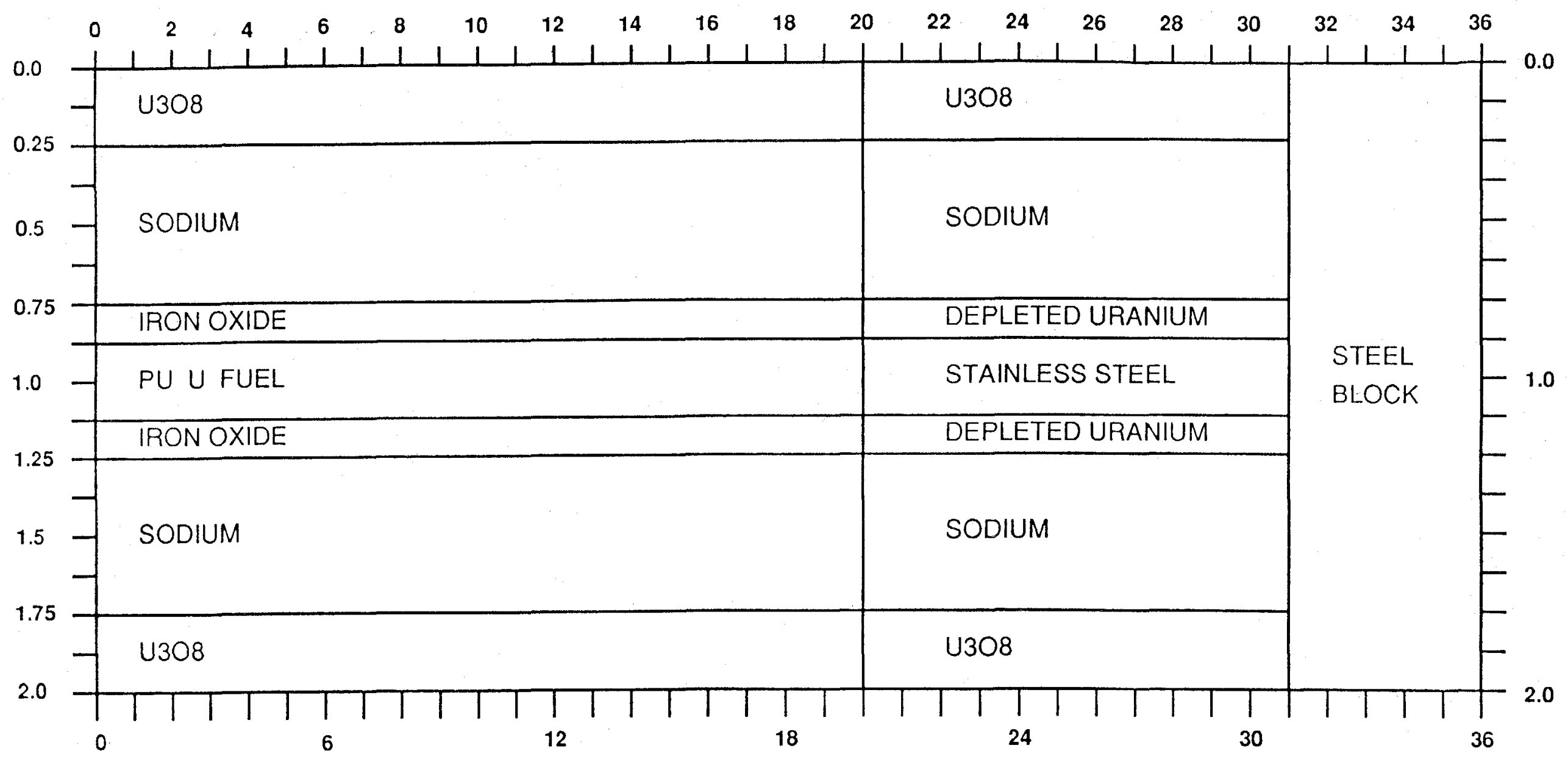

Fig. 2.1 Loading Pattern for Single-Fuel-Column Drawers without Internal Blanket. 


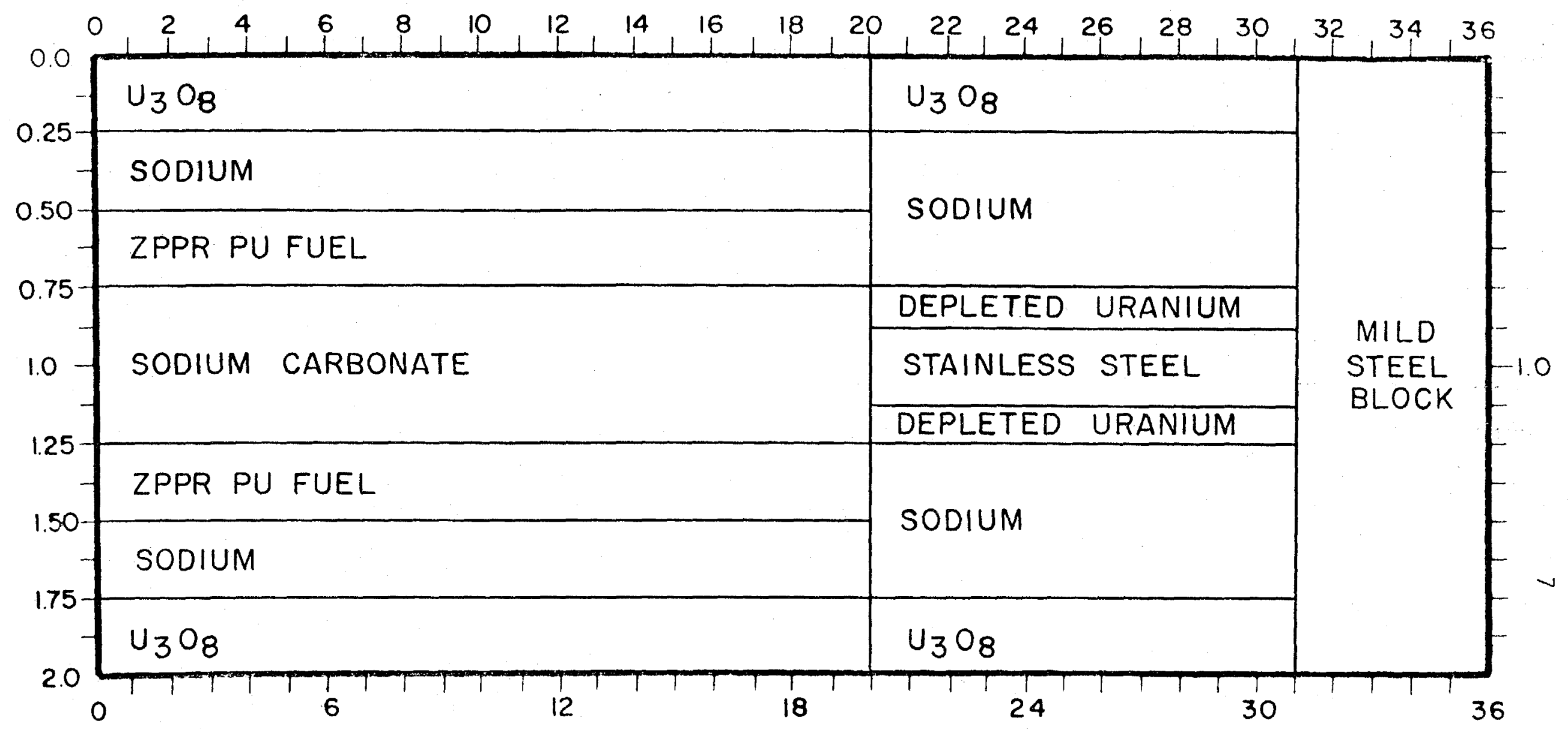

Fig. 2.2 Loading Pattern for Double-Fuel-Column Drawers without Interna1 Blanket. 


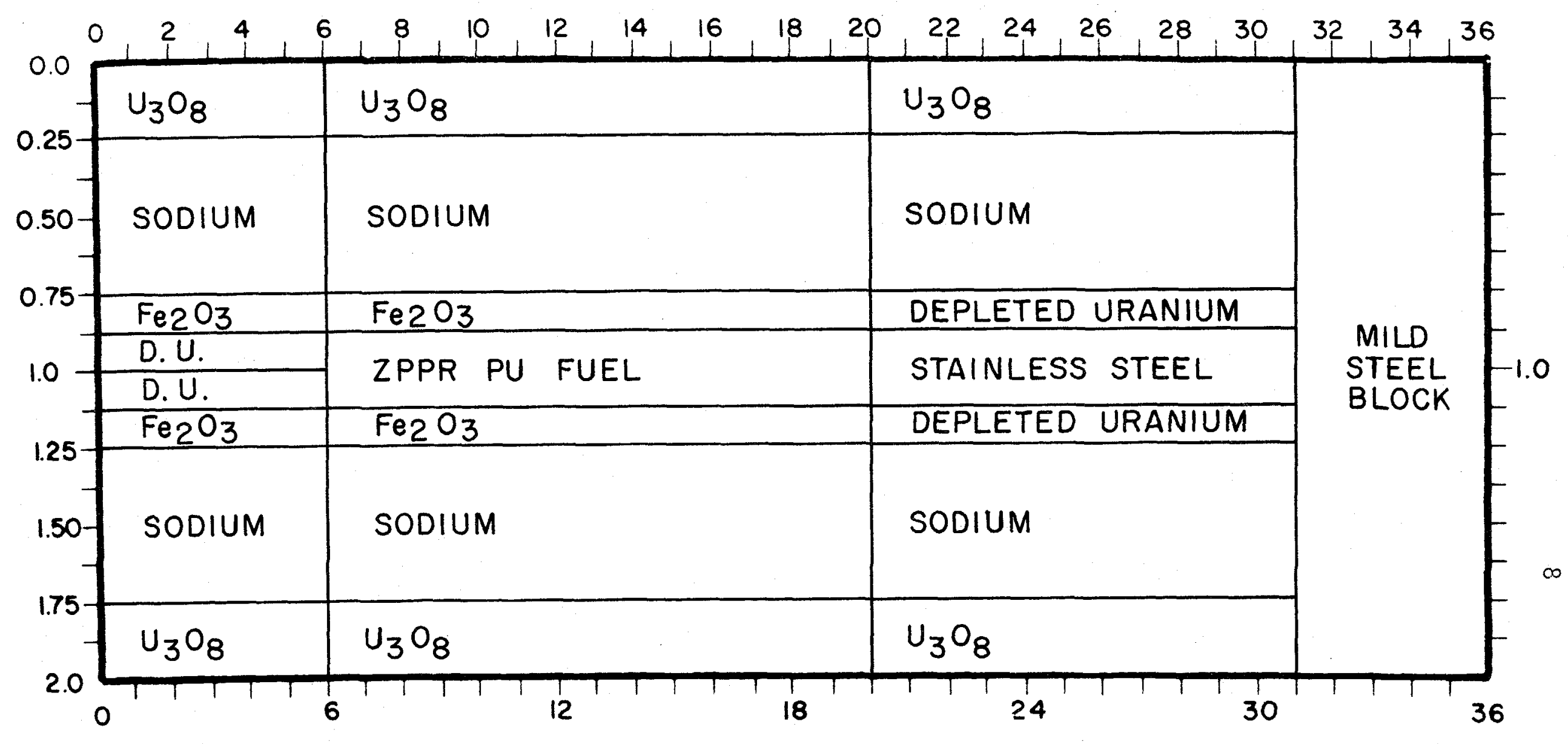

Fig. 2.3. Loading Pattern for Single-Fuel-Column Drawers with Internal Blanket Segment in ZPPR-17. 


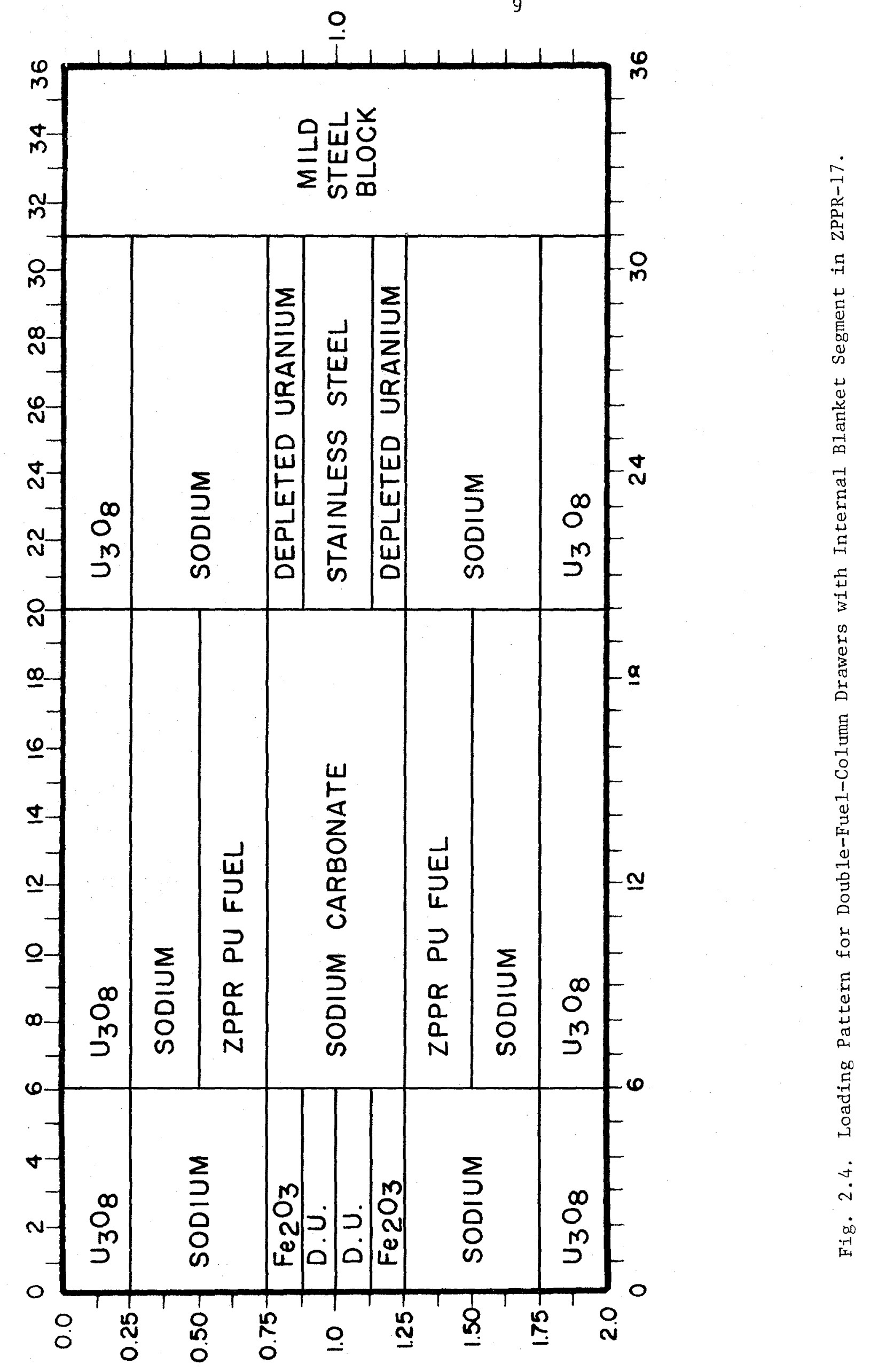




\section{EXPERIMENTAL TECHNIQUES}

Most of the experimental methods used in ZPPR-17 were the same as those described for the JUPITER-II program. ${ }^{8}$ The descriptions provided in reference 8 are applicable for $k$-effective, reaction rates, control rod worths, sodium void worths and small sample worths using the axial and radial tube oscillators. A minor modification was made to the way uncertainties are evaluated for reactivities produced by the LSFIT path of the McCRUNCH code. This modification was described in reference 4. Also, for the foil reaction rate measurements, the gamma ray counting system was recalibrated in irradiations in $2 P P R-17$.

Some new experimental techniques were developed at ZPPR since the JUPITER-II program. These new techniques have been aimed at evaluating the reactivity coefficients associated with material movements such as radial and axial expansion and assembly bowing. In addition, a new device called the plate-column oseillator drawer was developed to measure the worth of various core components. These new devices are described below. A brief description of the gamma ray dose measurements is also provided.

\subsection{Plate-Column Oscillators}

The plate-column oscillator is a new device developed at ZPPR which moves a column of plates axially within a drawer to provide material worth information for reactor constituents. In ZPPR-17, these devices were used to measure worths of fuel, $\mathrm{U}_{3} \mathrm{O}_{8}, \mathrm{Fe}_{2} \mathrm{O}_{3}$ and sodium.

The oscillators are made from two normal $920 \mathrm{~mm}-1$ ong drawers welded together at their ends to form a single $1.84 \mathrm{~m}$-long drawer. The front of this drawer can accommodate up to $660 \mathrm{~mm}$ of normal core and axial blanket material. The back of the drawer contains a small computercontrolled electric motor, a position encoder, and a coupling to a drive mechanism which is in turn attached to a column of material in the front of the drawer. This system allows the column to be oscillated up to $660 \mathrm{~mm}$ axially within the drawer. To assure free movement of the column, one $3.18 \mathrm{~mm}$-wide column of material is normally omitted from the drawer loading 
and a tray is introduced as a guide for the moving column. The tray is made from a $0.127 \mathrm{~mm}$-thick, $50 \mathrm{~mm}$ wide strip of stainless steel. Plates in the moving column are normally held together with mylar tape. Further details of the plate column oscillators used in ZPPR-17 are provided in reference 3 .

\subsection{Axial Expansion Oscillator}

The axial expansion oscillator is a device which simulates uniform axial expansion by creating a few discrete gaps between segments of a special drawer. The device can fit in almost any matrix tube. In the ZPPR17 measurements, three $6.35 \mathrm{~mm}$-wide gaps were created in the core region of the drawer. The axial distances from the matrix interface to the points where the drawer segments separate were $152 \mathrm{~mm}, 305 \mathrm{~mm}$ and $457 \mathrm{~mm}$. The width of the gaps was limited by attaching the drawer segments together with mylar tape. The gaps were created by pulling on the back end of the drawer while the front end was anchored. Axial motion was provided by a doubleacting air cylinder with a connecting rod that attaches to the back of the last drawer segment. A linear potentiometer measured the total expansion.

Statistical uncertainties of the axial expansion reactivities derived in ZPPR-17 were less than $1 \%$. However, measurements in previous cores indicated an uncertainty of about $3 \%$ based on reproducibility in repeat measurements.

\subsection{Bowing Oscillator}

The bowing oscillator is a new device which simulates fuel assembly bowing by shifting the core section of one drawer about $1.4 \mathrm{~mm}$ within the matrix tube. Air driven cams under the four corners of the moveable drawer segment push the drawer up into the cooling air gap. Motion is measured by linear potentiometers in three of the drawer corners. Uncertainties of the bowing reactivity measurements made in ZPPR-17 were $3.5 \%$. More detailed descriptions of the device and the ZPPR-17 experiments are given in reference 3 . 


\subsection{Gamma Ray Dose Measurements}

Gamma ray doses were measured in ZPPR-17 as a function of axial and radial location using stainless-steel-encapsulated thermoluminescent dosimeters (TLDS). The LiF TLDs were $1 \times 1 \times 6 \mathrm{~mm}$ and were placed in holes in stainless steel cylinders. The TLDs record neutron capture in the LiF as well as gamma dose in the steel cylinders. Calculation and experiment are compared for this "total dose". Using the calculated fluxes and the neutron sensitivities of the TLDs gives estimates of the neutron contribution to the TLD excitation of $18 \%$ in the core and $22 \%$ in the blanket.

Measured gamma dose varies significantly with the specific TLD location in a drawer. In-cell variations were calculated in $2 P P R-17$ and the results used to provide "cell averaging factors" to convert the dose at a specific TLD location to an average dose over the cell. Variations through a cell were dependent upon neighboring cell-types and multi-drawer cell models were used to obtain the most appropriate factors. Cell factors varied between 0.96 and 1.10 . Several in-cell measurements were also made which verified the accuracy of the calculated distributions.

Uncertainties in relative dose measurements are about $2 \%$ and an additional $2 \%$ uncertainty is due to calibration. Calibration of the TLDs in their stainless steel sleeves is made with a ${ }^{60}$ Co source. 


\section{CALCULATION METHODS}

\subsection{Introduction}

Calculations for ZPPR-17 used ENDF/B-V.2 nuclear data. The cross sections were processed through the $\mathrm{MC}^{2}$ and SDX codes, using methods consistent with those used for recent analysis of ZPPR and for benchmark cores, to produce a 21 group cross sections library for reactor calculations.

Two cross section libraries were produced for ZPPR-17. The first used asymptotic cell processing as described in Section 4.2. An improved cross section library used group-dependent bucklings in the cell calculations with a method analagous to that used for analysis of ZPPR-13 (buckling-recycle). This method and comparisons with analysis using asymptotic cell processing are described in section 4.3 .

The improved cross sections were used for analysis of reaction rates, small sample worths and related data. Calculations of control rod worths and sodium void reactivity used the original asymptotic-cell data since the analysis was quite advanced (in a temporal sense) when the newer cross sections became available.

The reference calculation method for ZPPR 17 used an xyz model with nodal diffusion and nodal transport solutions. The models used detailed compositions for each drawer master (with the exception of asymmetric fission chamber and thermocouple drawers) and were set up automatically from the ZPPR database. Calculations were also made using finite-difference diffusion in $x y z$ and $r z$ geometry, using the TWODANT transport code and using the TRITAC xyz transport code from Osaka University. Details of the calculation models are given in Section 4.4 .

Because of the large number of control rod measurements and the additional calculations required to obtain detector efficiencies and effective source rates, for $Z P P R-17 A$ and $-17 B$ these calculations were made in 6 group condensations of the 21 group library. Group collapse was made 
using xyz models for each series. Details are given in the control rod sections of this report.

\subsection{Multigroup Cross Section Processing}

Calculations of the ZPPR cell heterogeneities used a 230 group intermediate library with the SDX code and collapse of the cell-average data to 21 groups was done in a one-dimensional diffusion model. The energy structure of the cross section libraries is shown in Table 4.1 .

The 230 group library was collapsed from a single spectrum calculated with the $\mathrm{MC}^{2}$-II code in 2082 groups. This used a homogeneous composition derived for a mixture of single- and double-fuel column drawers in the proportion 65:35, and employed a buckling search to critical $\left(B^{2}=14.52 \mathrm{M}^{-2}\right)$. Input parameters chosen were consistent $\mathrm{P} 1$ spectrum calculation, "screened" diluent isotopes ( $\mathrm{Na}, \mathrm{Fe}, \mathrm{Cr}, \mathrm{Ni}, \mathrm{Mn}, \mathrm{Mo}$ ) 20 resolved resonances tested for overlap treatment and thermal group cross sections included from the dataset THERM.

The cell calculations with SDX used standard modelling in onedimension, preserving mid-cell densities and thicknesses for heavy metal plates. Following treatment of resonance shielding and flux fine structure, the actual homogeneous compositions were used in the final flux calculations for group collapse. The calculations were made for infinite arrays of cells in each case. The following cells were processed:

\footnotetext{
(i) Single-column fuel - normal cell (SCFN), voided cell (SCFV)

(ii) Double-column fuel - normal cell (DCFN), voided cell (DCFV)

(iii) Internal blanket - normal cell (IBKN), voided cell (IBKV)

(iv) Axial blanket - normal cell (ABKN), voided cell (ABKV)

(v) Radial blanket - normal cell (RBKN)

(vi) Single-column fuel cell with boron blade (PSR)
}

The PSR cell was used to obtain $\mathrm{B}_{4} \mathrm{C}$ data averaged over a drawer to model shim rods inserted for reaction rate measurements. The fuel cells were processed with a buckling search to critical and the blanket cells were 
processed with a zero buckling. Axial reflectors were treated as homogeneous regions, designated ARI for the $5 \mathrm{in.}$. iron block in the drawers and ARS for the 6 in. steel block loaded in the matrix.

Collapse of the cross sections from 230 groups to 21 groups was done with a slab model of the inner core region shown in Fig. 4.1. The arrangement of cells in the inner core region, SCFN, DCFN, SCFN, was chosen to simplify the collapse procedure within the SEF1D Module of the SDX code. Subsequent reactor calculations used cross sections collapsed in zone 2 for all single-fuel-column drawers and cross sections collapsed in zone 3 for all double-fuel-column drawers. Cross sections for sodium voided cells were collapsed in the same model but with cross sections IBKV, SCFV, etc., used in the internal blanket, core and axial blanket - i.e. in completely voided spectra. Cross sections for the radial blanket and radial reflector were also collapsed in the same model, with these cells replacing the axial blanket and reflector.

Anisotropic diffusion coefficient modifiers were generated by the Benoist method for the cell types listed above, including voided cells for the internal blanket, fuel drawers and the axial blanket. These cell models used plate compositions averaged over the lattice pitch rather than mid-cell compositions.

\subsection{Improved Cross Sections for ZPPR-17}

The effort to generate a new set of broad group microscopic cross sections for $Z P P R-17$ was stimulated by disappointing $C / E$ results for a number of experiments. One problem was a shift in reaction rate $\mathrm{C} / \mathrm{E}$ between core and blanket and even between single-fuel-column (SFC) and double-fuel-column (DFC) drawers within the core. Similarly, there was a mismatch between core and blanket small-sample worth C/Es for some traverses.

Some theoretical considerations suggested that improved cross sections might help remedy the $\mathrm{C} / \mathrm{E}$ problems. The original cross sections accounted for the close proximity of different cell types only at the stage 
of collapsing from 230 to 21 energy groups; in the resonance calculations and the cell homogenization the cells were treated as asymptotic. In the case of blanket cells the zero buckling eigenvalue approach used for the homogenization leads to a substantially distorted in-cell flux distribution. In addition, the particular models used for two blanket cells resulted in excessive ${ }^{2{ }^{3} \mathrm{U}}$ resonance self shielding by equivalence theory.

For the new cross sections the buckling recycle approach was used. This is the same method as used for the radially heterogeneous assembly ZPPR-13. (The available alternative, one-dimensional multidrawer cell models, is considerably more difficult to implement and, because of the many combinations of drawer types in multiple dimensions, probably no more accurate.) For each cell type the group-dependent bucklings, averaged over all occurences of that cell in ZPPR-17A, were extracted from a DIF3D XYZ geometry, 21 group, nodal diffusion theory assembly calculation.

With cell interaction accounted for by bucklings, each cell type was processed separately with the SDX code. The bucklings entered into the SDX calculations of resonance self shielding, cell homogenization and energy collapse from 230 to 21 groups.

Additional steps were taken to improve the cross sections. A 230 group base library was created for the SDX blanket cell calculations. This was done using the internal blanket composition and bucklings in the $\mathrm{MC}^{2}-2$ code. (The original base library was still considered appropriate for the core cells.) Also the blanket cell models were redefined to minimize the equivalence theory resonance self shielding errors. In addition a heterogeneous resonance self shielding treatment was used for sodium in the core and blanket cells. (Originally it was homogeneous.)

Cell average, plate and detector cross sections were saved for each of the cells. The cell average cross sections have the same names as their counterparts in the original set. The first two characters of the name identify the isotope and the next two identify the cell. For example, cell average ${ }^{238} \mathrm{U}$ in the internal blanket is called U8IB. Detector cross section names have $H$ as a fifth character (e.g., U8IBH) and plate cross 
section names have a number identifying the plate as a fifth character (e.g., U8IB2). Cell average cross sections are used with homogenized cell models. Plate cross sections are used for sodium and heavy metal isotopes in plate-by-plate cell models. Detector cross sections are used for the remaining isotopes in plate cell models, and also are often the best choice available for small-sample worths and foil reaction rates.

For the ${ }^{23 y} \mathrm{Pu}$ small sample and activation foils in blanket and reflector regions, detector cross sections are not a good choice. This is because resonance self shielding in the foil and sample are important there, while the $\mathrm{Pu}$ detector cross sections have no self shielding there. Accordingly, appropriately shielded cross sections were produced for $\mathrm{Pu}$ activation foils (named P9IBR) and for the reactivity sample PU-30. The activation foil cross sections were created using a slab foil, with the correct mean chord length, surrounded with a large region of homogeneous inner blanket and imposing the inner blanket bucklings.

There were two attempts to generate cross sections for PU-30. The first followed the same procedure used for the activation foils, yielding cross sections for ${ }^{239} \mathrm{Pu},{ }^{240} \mathrm{Pu}$ and ${ }^{241} \mathrm{Pu}$ whose names end in IBW. When used with a broad group sample size correction factor (from the SARCASM code) they gave worths that appear to be overshielded. To overcome this, cross sections were generated that have all the size effects built into them; they are the cell average cross sections from a cell that had PU-30 represented as an infinite cylinder at the center, surrounded by a large annulus of homogeneous blanket and with the inner blanket bucklings imposed (names end in IC).

Boundary region cross sections also were created for some cells near region interfaces. Group-dependent bucklings were extracted for each of the following cases: a SFC cell next to the inner blanket, a DFC cell next to the inner blanket, an inner blanket cell next to the core and an inner blanket cell not near the core. SDX was then rerun for these cells with the boundary bucklings. For these cases characters three through 5 of the cross section name are used to identify the cell. For the cases cited above the characters are SFN, DFF, IBE and IBI, respectively. 
The effect of the new cross sections on eigenvalue is shown in Table 4.2 for three models of ZPPR-17A. The first model includes only the central $3 \times 3$ array of drawers, with reflective boundary conditions at $X$ and $Y$ boundaries. This is an economical testbed. The other two models use a full assembly representation, one with diffusion theory and the other with transport theory. With all three models it is seen that the new region average cross sections lower the eigenvalue by a little more than $0.1 \%$. When the boundary region cross sections are also included in the first model, the effect is cut in half. The boundary cross sections are cumbersome to use in full assembly models, and their impact does not appear to be large enough to be worth the effort. In any case the cross section effect on eigenvalue is small.

Axial reaction rate traverses were computed for the three cross section options with the first model. The results are given in Table 4.3 . Except for ${ }^{239} \mathrm{Pu}$ fission (F9) there are modest changes in the inner blanket and almost no change elsewhere. For Fg there is a large change in the inner blanket, which is due to the resonance shielding treatment discussed earlier. This is illustrated further by Table 4.4, which shows the sensitivity to the reaction cross section type used in the inner blanket.

Using the nodal transport model of the full assembly, reaction rate $\mathrm{C} / \mathrm{ES}$ were computed with both the old and the new region average cross sections. Generally the C/Es improved with the new cross sections, but not as much as had been hoped. The difference between SFC and DFC C/ES was reduced from $0.9 \%$ to $0.2 \%$ for $\mathrm{F} 9$, reduced from $2.4 \%$ to $1.8 \%$ for ${ }^{238} \mathrm{U}$ fission and increased from $2.8 \%$ to $3.6 \%$ for ${ }^{238} \mathrm{U}$ capture. There were similar changes in the $\mathrm{C} / \mathrm{E}$ difference between the inner blanket and the core.

Small sample worth traverses also were computed with both cross section sets. Generally the worths changed one to two percent. Pu-30 worth in the blankets and reflectors changed much more because the sample cross sections in the new set were used.

In summary, the new cross sections generally have removed only a fraction of the $\mathrm{C} / \mathrm{E}$ problems. One conjecture as to why the improvements 
were not greater has to do with neutron leakage between different cells. Although the region-averaged, group-dependent bucklings should produce a realistic collapsing spectrum and, at least for the blanket, an improved in-cell flux distribution, they do not force preservation of leakage between unlike celis. As currently implemented, the multidrawer cell option in the SDX code does not do this either. This is an area that probably warrents some investigation.

\subsection{Reactor Models}

The reference calculation model for ZPPR-17 assumes 1/8-core symmetry (one-quarter $x y$, one-half $z$ ). The model follows the reactor loading in detail with the compositions of the principal drawer masters included individually. Loadings of fission chamber and thermocouple drawers were not represented since these were not loaded symmetrically in the reactor. These drawers were assigned compositions of the appropriate standard drawers. The model was constructed automatically from the ZPPR database through the NIPPER code.

Figures 4.2 to 4.4 show the xy planes of the calculation model. The entries in each matrix position are the individual drawer master numbers. Axial composition slices were chosen at $153.16 \mathrm{~mm}$ (internal blanket boundary), at $508.76 \mathrm{~mm}$ (core/axial blanket interface), at $788.16 \mathrm{~mm}$ (axial blanket/reflector interface), at $918.21 \mathrm{~mm}$ (iron block reflector to back of drawer), at at $1070.61 \mathrm{~mm}$ (stainless steel reflector). The zone boundaries are shown in an $\mathrm{rz}$ diagram of ZPPR-17A fig. 4.5 .

Average zone compositions, in units of $10^{24}$ atoms $\mathrm{cm}^{-3}$, are given in Tables 4.5 to 4.7 for the axial slices defined above. These compositions are derived for a weighted average of all the individual drawer masters in a zone (excluding fission chamber and thermocouple drawers). The fuel region above the internal blanket has been labelled inner core, although as can be seen, the compositions are nominally the same as those in the "outer core" region. Compositions for the mixtures of single and double-fuel-column drawers are also included in the table. The compositions are given for the axial slices described above, labelled in inches for simplicity. The 
composition data in Tables 4.5 to 4.7 are suitable for use in rz models but, as noted above, the reference xyz models use individual drawer compositions.

Cross sections in 6 groups were collapsed using zone spectra from the 21 group xyz calculations. Benoist diffusion coefficients were also collapsed for the same zones using a $D \phi$ weighting. 
TABLE 4.1 Energy Structure of the Cross Section

Sets used for ZPPR-17A Analysis

\begin{tabular}{|c|c|c|c|c|}
\hline Energy Boundary ${ }^{a}$ & $\begin{array}{c}\text { 6-Group } \\
\text { Number }\end{array}$ & $\begin{array}{l}21-\text { Group } \\
\text { Number }\end{array}$ & $\begin{array}{l}\text { 230-Group } \\
\text { Number }\end{array}$ & $\begin{array}{l}\text { 2082-Group } \\
\text { Number }\end{array}$ \\
\hline $14.191 \mathrm{MeV}$ & & & & \\
\hline 1. 6.065 & & 1 & 36 & 102 \\
\hline 3.679 & & 2 & 49 & 162 \\
\hline 2.231 & & 3 & 66 & 222 \\
\hline 4. 1.353 & 1 & 4 & 80 & 282 \\
\hline 5. $820.9 \mathrm{keV}$ & & 5 & 93 & 342 \\
\hline 6.497 .9 & & 6 & 105 & 402 \\
\hline 7.302 .0 & & 7 & 116 & 462 \\
\hline 8. 183.2 & & 8 & 128 & 522 \\
\hline 9. 111.1 & & 9 & 140 & 582 \\
\hline 10. 67.38 & 2 & 10 & 144 & 642 \\
\hline 11. 40.87 & & 11 & 150 & 702 \\
\hline 12. 24.79 & & 12 & 157 & 762 \\
\hline 3. 15.03 & & 13 & 164 & 822 \\
\hline 4. 9.119 & 3 & 14 & 168 & 882 \\
\hline 5.531 & & 15 & 172 & 942 \\
\hline 3.355 & & 16 & 178 & 1002 \\
\hline 17. 2.035 & & 17 & 185 & 1062 \\
\hline 18. $\quad 1.234$ & & 18 & 191 & 1122 \\
\hline $19.748 .5 \mathrm{eV}$ & & & 197 & 1182 \\
\hline 20.454 .0 & 4 & 19 & 199 & 1242 \\
\hline 21. 275.4 & & & 201 & 1302 \\
\hline 22. 167.0 & & & 203 & 1362 \\
\hline 23. 101.3 & & & 205 & 1422 \\
\hline 24. 61.44 & 5 & 20 & 207 & 1482 \\
\hline 25. 37.27 & & & 209 & 1542 \\
\hline 26. 22.60 & & & 211 & 1602 \\
\hline 27.5 .04 & & & 217 & 1782 \\
\hline 28. Thermal & 6 & 21 & 230 & 2082 \\
\hline
\end{tabular}

${ }^{a}$ The 28 energy boundaries are those of the cross section used in previous JUPITER analysis.

${ }^{b}$ The $M C^{2}-$ II library used 2082 groups with lethargy width of $1 / 120$. The SDX intermediate library had 230 groups with a variable lethargy width tailored to treat the narrow resonances in structural materials. 


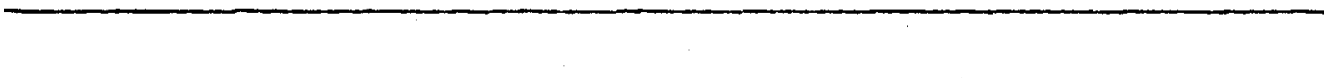

$\mathrm{k}$

Nodal Diffusion XYZ Model of Central $3 \times 3$ Drawers

Old cross sections

0.956980

New region avg. cross sections

0.955319

$>-.0017$

New region avg. and boundary cross sections

0.956084

$>+.0008$

Nodal Diffusion XYZ Model of Full Assembly, with Benoist, no PSRs

Old cross sections

0.987060

New region avg. cross sections

0.985825

Nodal Transport XYZ Model of Full Assembly, with Benoist, with PSRs

Old cross sections

0.990930

New region avg. cross sections

0.989749 
TABLE 4.3 Axial Reaction Rate Traverses with Old and New Cross Sections ${ }^{a}$

\begin{tabular}{|c|c|c|c|c|c|c|c|c|c|c|}
\hline \multirow[b]{3}{*}{ Cross } & \multirow[b]{3}{*}{ Sections: } & \multicolumn{9}{|c|}{ Normalized Microscopic Reaction Rates ${ }^{b}$} \\
\hline & & \multicolumn{3}{|c|}{${ }^{238} \mathrm{U}$ Fission } & \multicolumn{3}{|c|}{${ }^{2{ }^{8} \mathrm{U} \text { Capture }}$} & \multicolumn{3}{|c|}{${ }^{239} \mathrm{Pu}$ Fission } \\
\hline & & Old & $\begin{array}{l}\text { Avg. } \\
\text { Only }\end{array}$ & $\begin{array}{l}\text { Avg. } \\
\text { and } \\
\text { Bdry. }\end{array}$ & Old & $\begin{array}{l}\text { Avg. } \\
\text { Only }\end{array}$ & $\begin{array}{l}\text { Avg. } \\
\text { and } \\
\text { Bdry. }\end{array}$ & 01d & $\begin{array}{l}\text { Avg. } \\
\text { Only }\end{array}$ & $\begin{array}{l}\text { Avg. } \\
\text { and } \\
\text { Bdry. }\end{array}$ \\
\hline 1 & IBK & 3.00 & 2.88 & 2.89 & 3.00 & 2.99 & 2.95 & 3.00 & 3.15 & 3.19 \\
\hline 2 & IBK & 4.88 & 4.70 & 4.70 & 2.92 & 2.91 & 2.94 & 2.97 & 3.09 & 3.11 \\
\hline 3 & SFC & 8.25 & 8.25 & 8.30 & 2.86 & 2.89 & 2.90 & 2.81 & 2.81 & 2.83 \\
\hline 4 & SFC & 10.66 & 10.68 & 10.66 & 2.75 & 2.77 & 2.77 & 2.89 & 2.88 & 2.88 \\
\hline 5 & SFC & 10.07 & 10.07 & 10.05 & 2.51 & 2.52 & 2.52 & 2.67 & 2.67 & 2.67 \\
\hline 6 & SFC & 7.13 & 7.13 & 7.13 & 2.22 & 2.23 & 2.23 & 2.24 & 2.24 & 2.24 \\
\hline 7 & ABK & 2.84 & 2.75 & 2.75 & 1.84 & 1.86 & 1.86 & & & \\
\hline 8 & $A B K$ & 0.65 & 0.63 & 0.63 & 1.28 & 1.28 & 1.28 & & & \\
\hline
\end{tabular}

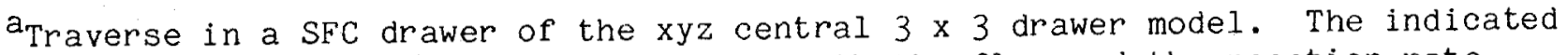
cross sections were used for computing both the flux and the reaction rate.

$b_{\text {Each }}$ reaction type is normalized such that the value at $Z$ mesh 1 obtained using the old cross sections is 3.00 . 
TABLE 4.4 Sensitivity of ${ }^{23 y} \mathrm{Pu}$ Fission in the Inner Blanket to Cross Section Type

\begin{tabular}{|c|c|c|}
\hline & Cross Section used for Reaction Rate ${ }^{a}$ & Normalized \\
\hline Name & Type & $2^{2{ }^{9}} \mathrm{Pu}(\mathrm{n}, \mathrm{f})$ \\
\hline P9IBR & $\begin{array}{l}\text { Activation foil in homogeneous IBKT with avg. } \mathrm{B}_{\mathrm{g}}^{2} \\
\text { (most appropriate available) }\end{array}$ & 1.000 \\
\hline $\mathrm{P9CO}$ & $\begin{array}{l}\text { "Detector" in homogeneous average core } \\
\text { (used in earlier reaction rate work) }\end{array}$ & 0.946 \\
\hline P9IBW & Pu-30 equivalent slab in homog. IBKT with avg. $\mathrm{B}_{\mathrm{g}}^{2}$ & 0.855 \\
\hline P9IC & $\begin{array}{l}\text { Cell avg., cell with } \mathrm{Pu}-30 \text { cylinder in homog. } \\
\text { blanket, with avg. } \mathrm{B}_{\mathrm{g}}^{2}\end{array}$ & 0.876 \\
\hline P9IBH & Detector in het. IBKT with avg. $\mathrm{B}_{\mathrm{g}}^{2}$ & 1.070 \\
\hline P9IBIH & Detector in het. IBKT with interior $\mathrm{B}_{\mathrm{g}}^{2}$ & 1.079 \\
\hline P9IBEH & Detector in het. IBKT with edge $\mathrm{B}_{\mathrm{g}}^{2}$ & 1.052 \\
\hline P9DFH & Detector in het. DFC cell with avg. DFC $\mathrm{B}_{\mathrm{g}}^{2}$ & 0.939 \\
\hline P9SFH & Detector in het. $S F C$ cell with avg. $S F C B_{g}^{2}$ & 0.970 \\
\hline P9SF & SFC cell avg. with avg. SFC $\mathrm{B}_{\mathrm{g}}^{2}$ & 0.861 \\
\hline P9SF2 & Fuel plate in het. SFC cell with avg. SFC $B_{g}^{2}$ & 0.898 \\
\hline
\end{tabular}

${ }^{a}$ Same flux used for all cases.

Resolved resonance range is 1 to $301 \mathrm{eV}$

Unresolved resonance range is $301 \mathrm{eV}$ to $25 \mathrm{keV}$

Contribution to P9IBR $\mathrm{f}^{49}$ at $\mathrm{z}=0$ to $7.6 \mathrm{~cm}$

$0 \mathrm{eV}$ to $61 \mathrm{eV}($ GP. 21$) \quad 4.1 \%$

$61 \mathrm{eV}$ to $454 \mathrm{eV}($ GP.20) $18.9 \%$

$454 \mathrm{eV}$ to $24.8 \mathrm{keV}$ (GPS. 13-19) $35.7 \%$

$58.7 \%$ 
TABLE 4.5 ZPPR Assembly 17A: Atom Densities by Zone

\begin{tabular}{|c|c|c|c|c|c|c|}
\hline Isotope & $\begin{array}{c}\text { Central } \\
\text { Blanket } \\
\text { SFC } \\
0-6 \\
\end{array}$ & $\begin{array}{c}\text { Central } \\
\text { Blanket } \\
\text { DFC } \\
0-6 \\
\end{array}$ & $\begin{array}{c}\text { Inner Core } \\
\text { SFC } \\
6-20 \\
\end{array}$ & $\begin{array}{c}\text { Inner Core } \\
\text { DFC } \\
6-20 \\
\end{array}$ & $\begin{array}{c}\text { Axial } \\
\text { Blanket } \\
20-31 \\
\end{array}$ & $\begin{array}{l}\text { Reflector } \\
\text { Iron } \mathrm{Bl} \text { ock } \\
\quad 31-36 \\
\end{array}$ \\
\hline C & 0.0000333 & 0.0000331 & 0.0000331 & 0.0022784 & 0.0000532 & 0.0005889 \\
\hline 0 & 0.0136987 & 0.0136964 & 0.0137650 & 0.0155776 & 0.0088214 & -- \\
\hline $\mathrm{Na}$ & 0.0092061 & 0.0091685 & 0.0092879 & 0.0087252 & 0.0092111 & -- \\
\hline $\mathrm{Si}$ & 0.0001433 & 0.0001458 & 0.0001570 & 0.0001822 & 0.0001925 & 0.0001231 \\
\hline Al & 0.0000029 & 0.0000029 & 0.0000040 & 0.0000062 & 0.0000029 & -- \\
\hline Mn & 0.0002046 & 0.0002019 & 0.0002282 & 0.0002671 & 0.0003416 & 0.0006795 \\
\hline $\mathrm{Cr}$ & 0.0023927 & 0.0023712 & 0.0026610 & 0.0031593 & 0.0041613 & 0.0020617 \\
\hline $\mathrm{Fe}$ & 0.0118901 & 0.0117862 & 0.0128337 & 0.0111569 & 0.0147819 & 0.0758126 \\
\hline $\mathrm{Ni}$ & 0.0010320 & 0.0010430 & 0.0011633 & 0.0014326 & 0.0017657 & 0.0008384 \\
\hline $\mathrm{Cu}$ & 0.0000274 & 0.0000319 & 0.0000294 & 0.0000365 & 0.0000446 & 0.0000276 \\
\hline Mo & 0.0000129 & 0.0000150 & 0.0002395 & 0.0004633 & 0.0000348 & 0.0000138 \\
\hline U5 & 0.0000178 & 0.0000178 & 0.0000127 & 0.0000182 & 0.0000179 & -- \\
\hline U8 & 0.0081368 & 0.0081355 & 0.0058393 & 0.0082951 & 0.0081563 & - \\
\hline P8 & $-\infty$ & -- & 0.0000005 & 0.0000010 & -- & -- \\
\hline P9 & - & -- & 0.0008875 & 0.0017529 & - & -- \\
\hline PO & - & -- & 0.0001175 & 0.0002314 & - & -- \\
\hline P1 & -- & -- & 0.0000074 & 0.0000142 & -- & -- \\
\hline P2 & - & -- & 0.0000019 & 0.0000037 & -- & -- \\
\hline A1 & - & -- & 0.0000109 & 0.0000210 & - & - \\
\hline $\mathrm{P}$ & 0.0000053 & 0.0000047 & 0.0000053 & 0.0000047 & 0.0000101 & 0.0000236 \\
\hline S & 0.0000010 & 0.0000013 & 0.0000010 & 0.0000016 & 0.0000081 & 0.0000313 \\
\hline $\mathrm{Cl}$ & 0.0000003 & 0.0000003 & 0.0000003 & 0.0000060 & 0.0000003 & -- \\
\hline $\mathrm{Ca}$ & 0.0000021 & 0.0000021 & 0.0000021 & 0.0000010 & 0.0000021 & - \\
\hline Co & 0.0000008 & 0.0000047 & 0.0000008 & 0.0000043 & 0.0000020 & 0.0000014 \\
\hline
\end{tabular}


TABLE 4.5 (contd)

\begin{tabular}{|c|c|c|c|c|c|c|}
\hline Isotope & $\begin{array}{c}\text { Reflector } \\
\text { Stainless } \\
36-42 \\
\end{array}$ & $\begin{array}{l}\text { Outer Core } \\
\text { SFC } \\
0-6 \\
\end{array}$ & $\begin{array}{c}\text { Outer Core } \\
\text { DFC } \\
0-6 \\
\end{array}$ & $\begin{array}{c}\text { Outer Core } \\
\text { SFC } \\
6-20 \\
\end{array}$ & $\begin{array}{c}\text { Outer Core } \\
\text { DFC } \\
6-20 \\
\end{array}$ & $\begin{array}{c}\text { Axial } \\
\text { Blanket } \\
20-31\end{array}$ \\
\hline $\mathrm{C}$ & 0.0002143 & 0.0000332 & 0.0022752 & 0.0000331 & 0.0022870 & 0.0000532 \\
\hline 0 & - & 0.0136987 & 0.0155223 & 0.0137650 & 0.0156026 & 0.0088213 \\
\hline $\mathrm{Na}$ & -- & 0.0092447 & 0.0087164 & 0.0092246 & 0.0087434 & 0.0091835 \\
\hline Si & 0.0008629 & 0.0001553 & 0.0001800 & 0.0001575 & 0.0001817 & 0.0001925 \\
\hline$A 1$ & -- & 0.0000038 & 0.0000057 & 0.0000041 & 0.0000062 & 0.0000029 \\
\hline $\mathrm{Mn}$ & 0.0015241 & 0.0002267 & 0.0002609 & 0.0002294 & 0.0002654 & 0.0003415 \\
\hline $\mathrm{Cr}$ & 0.0150441 & 0.0026487 & 0.0030954 & 0.0026741 & 0.0031422 & 0.0041605 \\
\hline $\mathrm{Fe}$ & 0.0531084 & 0.0127704 & 0.0109304 & 0.0128793 & 0.0110970 & 0.0147790 \\
\hline $\mathrm{Ni}$ & 0.0066621 & 0.0011599 & 0.0014039 & 0.0011699 & 0.0014240 & 0.0017656 \\
\hline $\mathrm{Cu}$ & 0.0000172 & 0.0000289 & 0.0000360 & 0.0000294 & 0.0000363 & 0.0000446 \\
\hline Mo & 0.0000083 & 0.0002402 & 0.0004697 & 0.0002398 & 0.0004685 & 0.0000349 \\
\hline U5 & -- & 0.0000126 & 0.0000183 & 0.0000127 & 0.0000183 & 0.0000179 \\
\hline U8 & -- & 0.0057976 & 0.0083514 & 0.0058140 & 0.0083529 & 0.0081562 \\
\hline P8 & -- & 0.0000003 & 0.0000010 & 0.0000004 & 0.0000010 & -- \\
\hline P9 & -- & 0.0008888 & 0.0017783 & 0.0008879 & 0.0017727 & -- \\
\hline PO & -- & 0.0001178 & 0.0002354 & 0.0001176 & 0.0002346 & -- \\
\hline P1 & -- & 0.0000064 & 0.0000145 & 0.0000066 & 0.0000145 & -- \\
\hline $\mathrm{P} 2$ & -- & 0.0000016 & 0.0000039 & 0.0000017 & 0.0000038 & -- \\
\hline$A 1$ & -- & 0.0000103 & 0.0000216 & 0.0000106 & 0.0000215 & - \\
\hline$P$ & -- & 0.0000053 & 0.0000047 & 0.0000053 & 0.0000047 & 00000101 \\
\hline$S$ & -- & 0.0000010 & 0.0000016 & 0.0000010 & 0.0000016 & 0.0000081 \\
\hline $\mathrm{Cl}$ & - & 0.0000003 & 0.0000060 & 0.0000003 & 0.0000061 & 0.0000003 \\
\hline $\mathrm{Ca}$ & -- & 0.0000021 & 0.0000010 & 0.0000021 & 0.0000010 & 0.0000021 \\
\hline Co & - & 0.0000008 & 0.0000046 & 0.0000008 & 0.0000044 & 0.0000020 \\
\hline
\end{tabular}


TABLE 4.5 (contd)

\begin{tabular}{|c|c|c|c|c|c|c|}
\hline Isotope & $\begin{array}{c}\text { Reflector } \\
\text { Iron Block } \\
31-36 \\
\end{array}$ & $\begin{array}{c}\text { Reflector } \\
\text { Stainless } \\
36-42 \\
\end{array}$ & $\begin{array}{c}\text { Radial } \\
\text { Blanket } \\
0-6 \\
\end{array}$ & $\begin{array}{c}\text { Radial } \\
\text { Blanket } \\
6-20 \\
\end{array}$ & $\begin{array}{c}\text { Axial } \\
\text { Blanket } \\
20-31\end{array}$ & $\begin{array}{c}\text { Reflector } \\
\text { Iron Block } \\
31-36 \\
\end{array}$ \\
\hline C & 0.0005882 & 0.0002143 & 0.0000322 & 0.0000323 & 0.0000323 & 0.0005928 \\
\hline 0 & -- & -- & 0.0223887 & 0.0225016 & 0.0224943 & -- \\
\hline $\mathrm{Na}$ & -- & - & 0.0042238 & 0.0042451 & 0.0042293 & -- \\
\hline $\mathrm{Si}$ & 0.0001244 & 0.0008629 & 0.0001388 & 0.0001397 & 0.0001397 & 0.0001164 \\
\hline $\mathrm{Al}$ & - & -- & 0.0000024 & 0.0000024 & 0.0000025 & - \\
\hline Mn & 0.0006804 & 0.0015241 & 0.0001976 & 0.0001990 & 0.0001990 & 0.0006745 \\
\hline $\mathrm{Cr}$ & 0.0020814 & 0.0150441 & 0.0023029 & 0.0023123 & 0.0023124 & 0.0019581 \\
\hline $\mathrm{Fe}$ & 0.0757474 & 0.0531084 & 0.0082260 & 0.0082639 & 0.0082641 & 0.0761486 \\
\hline $\mathrm{Ni}$ & 0.0008475 & 0.0066621 & 0.0009924 & 0.0009938 & 0.0009938 & 0.0007910 \\
\hline $\mathrm{Cu}$ & 0.0000276 & 0.0000172 & 0.0000282 & 0.0000285 & 0.0000285 & 0.0000273 \\
\hline Mo & 0.0000139 & 0.0000083 & 0.0000133 & 0.0000132 & 0.0000132 & 0.0000137 \\
\hline U5 & -- & - & 0.0000288 & 0.0000290 & 0.0000289 & - \\
\hline U8 & - & -- & 0.0132208 & 0.0132875 & 0.0132871 & -- \\
\hline P8 & - & -- & - & -- & - & - \\
\hline PQ & -- & - & - & - & - & - \\
\hline PO & -- & - & - & -- & -- & $-\infty$ \\
\hline P1 & -- & -- & -- & -- & -- & -- \\
\hline $\mathrm{P} 2$ & -- & - & - & -- & - & -- \\
\hline A1 & -- & -- & -- & -- & -- & - \\
\hline$P$ & 0.0000236 & -- & 0.0000050 & 0.0000051 & 0.0000051 & 0.0000234 \\
\hline$S$ & 0.0000313 & -- & 0.0000011 & 0.0000011 & 0.0000011 & 0.0000314 \\
\hline $\mathrm{Cl}$ & -- & -- & 0.0000001 & 0.0000001 & 0.0000001 & - \\
\hline $\mathrm{Ca}$ & - & - & 0.0000010 & 0.0000010 & 0.0000010 & -- \\
\hline $\mathrm{Co}$ & 0.0000015 & - & 0.0000018 & 0.0000017 & 0.0000017 & 0.0000012 \\
\hline
\end{tabular}


TABLE 4.5 (contd)

\begin{tabular}{|c|c|c|c|c|c|c|}
\hline Isotope & $\begin{array}{c}\text { Reflector } \\
\text { Stainless } \\
36-42 \\
\end{array}$ & $\begin{array}{c}\text { Radial } \\
\text { Reflector } \\
0-36 \\
\end{array}$ & $\begin{array}{c}\text { Blanket } \\
\text { Central } \\
(\mathrm{SFC}+\mathrm{DFC}) \\
0-6 \\
\end{array}$ & $\begin{array}{c}\text { Inner Core } \\
(\mathrm{SFC}+\mathrm{DFC}) \\
6-20 \\
\end{array}$ & $\begin{array}{l}\text { Outer Core } \\
(\mathrm{SFC}+\mathrm{DFC}) \\
0-6 \\
\end{array}$ & $\begin{array}{r}\text { Outer Core } \\
(\mathrm{SFC}+\mathrm{DFC}) \\
6-20 \\
\end{array}$ \\
\hline C & 0.0002143 & 0.0002510 & 0.0000332 & 0.0007739 & 0.0008016 & 0.0008056 \\
\hline 0 & -- & - & 0.0136981 & 0.0143632 & 0.0143237 & 0.0143948 \\
\hline $\mathrm{Na}$ & -- & -- & 0.0091938 & 0.0091023 & 0.0090636 & 0.0090597 \\
\hline Si & 0.0008629 & 0.0008767 & 0.0001441 & 0.0001653 & 0.0001638 & 0.0001658 \\
\hline Al & -- & -- & 0.0000029 & 0.0000047 & 0.0000045 & 0.0000048 \\
\hline $\mathrm{Mn}$ & 0.0015241 & 0.0013973 & 0.0002037 & 0.0002410 & 0.0002384 & 0.0002417 \\
\hline $\mathrm{Cr}$ & 0.0150441 & 0.0153821 & 0.0023857 & 0.0028254 & 0.0028018 & 0.0028346 \\
\hline $\mathrm{Fe}$ & 0.0531084 & 0.0546625 & 0.0118560 & 0.0122806 & 0.0121398 & 0.0122684 \\
\hline $\mathrm{Ni}$ & 0.0066621 & 0.0066801 & 0.0010357 & 0.0012522 & 0.0012436 & 0.0012570 \\
\hline $\mathrm{Cu}$ & 0.0000172 & 0.0000400 & 0.0000289 & 0.0000317 & 0.0000313 & 0.0000318 \\
\hline Mo & 0.0000083 & 0.0000368 & 0.0000136 & 0.0003133 & 0.0003189 & 0.0003182 \\
\hline U5 & - & $\ldots$ & 0.0000178 & 0.0000145 & 0.0000146 & 0.0000146 \\
\hline U8 & -- & -- & 0.0081365 & 0.0066497 & 0.0066729 & 0.0066842 \\
\hline P8 & -- & -- & -- & 0.0000007 & 0.0000006 & 0.0000006 \\
\hline P9 & -- & - & - & 0.0011731 & 0.0011937 & 0.0011912 \\
\hline PO & - & -- & - & 0.0001551 & 0.0001581 & 0.0001577 \\
\hline P1 & - & -- & -- & 0.0000096 & 0.0000092 & 0.0000094 \\
\hline P2 & -- & -- & - & 0.0000025 & 0.0000024 & 0.0000024 \\
\hline A1 & - & -- & -- & 0.0000142 & 0.0000142 & 0.0000143 \\
\hline $\mathrm{P}$ & -- & 0.0000458 & 0.0000051 & 0.0000051 & 0.0000051 & 0.0000051 \\
\hline S & - & 0.0000352 & 0.0000011 & 0.0000012 & 0.0000012 & 0.0000012 \\
\hline $\mathrm{Cl}$ & -- & $-\infty$ & 0.0000003 & 0.0000022 & 0.0000023 & 0.0000023 \\
\hline $\mathrm{Ca}$ & -- & -- & 0.0000021 & 0.0000017 & 0.0000017 & 0.0000017 \\
\hline Co & -- & 0.0000009 & 0.0000021 & 0.0000020 & 0.0000021 & 0.0000020 \\
\hline
\end{tabular}


TABLE 4.5 (contd)

\begin{tabular}{|c|c|}
\hline Isotope & $\begin{array}{r}\text { Empty } \\
\text { Matrix } \\
\end{array}$ \\
\hline $\mathrm{C}$ & 0.0000188 \\
\hline 0 & -- \\
\hline $\mathrm{Na}$ & -- \\
\hline Si & 0.0000683 \\
\hline Al & -- \\
\hline $\mathrm{Mn}$ & 0.0001059 \\
\hline $\mathrm{Cr}$ & 0.0011891 \\
\hline $\mathrm{Fe}$ & 0.0042791 \\
\hline $\mathrm{Ni}$ & 0.0004802 \\
\hline $\mathrm{Cu}$ & 0.0000172 \\
\hline Mo & 0.0000083 \\
\hline U5 & -- \\
\hline U8 & -- \\
\hline P8 & - \\
\hline P9 & $-\infty$ \\
\hline PO & -- \\
\hline $\mathrm{P} 1$ & -- \\
\hline P2 & - \\
\hline $\mathrm{A} 1$ & -- \\
\hline $\mathrm{P}$ & 0.0000028 \\
\hline$S$ & 0.0000007 \\
\hline $\mathrm{C} 1$ & -- \\
\hline $\mathrm{Ca}$ & -- \\
\hline Co & -- \\
\hline
\end{tabular}


TABLE 4.6 ZPPR Assembly 17B: Atom Densities by Zone

\begin{tabular}{|c|c|c|c|c|c|c|}
\hline Isotope & $\begin{array}{c}\text { Central } \\
\text { Blanket } \\
\text { SFC } \\
0-6 \\
\end{array}$ & $\begin{array}{c}\text { Central } \\
\text { Blanket } \\
\text { DFC } \\
0-6 \\
\end{array}$ & $\begin{array}{c}\text { Inner Core } \\
\text { SFC } \\
6-20 \\
\end{array}$ & $\begin{array}{c}\text { Inner Core } \\
\text { DFC } \\
6-20 \\
\end{array}$ & $\begin{array}{c}\text { Axial } \\
\text { Blanket } \\
20-31 \\
\end{array}$ & $\begin{array}{c}\text { Reflector } \\
\text { Iron Block } \\
31-36 \\
\end{array}$ \\
\hline C & 0.0000333 & 0.0000342 & 0.0000331 & 0.0022822 & 0.0000532 & 0.0005884 \\
\hline 0 & 0.0136987 & 0.0136964 & 0.0137650 & 0.0155893 & 0.0088213 & -- \\
\hline $\mathrm{Na}$ & 0.0091995 & 0.0091764 & 0.0092812 & 0.0087323 & 0.0092077 & - \\
\hline $\mathrm{Si}$ & 0.0001434 & 0.0001498 & 0.0001570 & 0.0001822 & 0.0001926 & 0.0001231 \\
\hline $\mathrm{Al}$ & 0.0000029 & 0.0000029 & 0.0000040 & 0.0000062 & 0.0000029 & - \\
\hline Mn & 0.0002047 & 0.0002081 & 0.0002282 & 0.0002672 & 0.0003414 & 0.0006786 \\
\hline $\mathrm{Cr}$ & 0.0023943 & 0.0024413 & 0.0026606 & 0.0031600 & 0.0041603 & 0.0020566 \\
\hline $\mathrm{Fe}$ & 0.0118957 & 0.0120382 & 0.0128326 & 0.0111595 & 0.0147764 & 0.0757381 \\
\hline $\mathrm{Ni}$ & 0.0010326 & 0.0010714 & 0.0011632 & 0.0014331 & 0.0017666 & 0.0008383 \\
\hline $\mathrm{Cu}$ & 0.0000274 & 0.0000329 & 0.0000294 & 0.0000365 & 0.0000448 & 0.0000277 \\
\hline Mo & 0.0000129 & 0.0000155 & 0.0002394 & 0.0004633 & 0.0000350 & 0.0000139 \\
\hline U5 & 0.0000178 & 0.0000178 & 0.0000127 & 0.0000182 & 0.0000179 & - \\
\hline U8 & 0.0081368 & 0.0081355 & 0.0058392 & 0.0082951 & 0.0081562 & \\
\hline P8 & - & - - & 0.0000005 & 0.0000010 & -- & \\
\hline P9 & - & - & 0.0008875 & 0.0017529 & - - & \\
\hline PO & - & - & 0.0001175 & 0.0002314 & - & \\
\hline P1 & -- & - & 0.0000073 & 0.0000140 & - & \\
\hline P2 & - & - & 0.0000019 & 0.0000037 & - & \\
\hline A1 & - & - & 0.0000109 & 0.0000211 & - & - - \\
\hline$P$ & 0.0000053 & 0.0000049 & 0.0000053 & 0.0000047 & 0.0000101 & 0.0000235 \\
\hline$S$ & 0.0000010 & 0.0000013 & 0.0000010 & 0.0000016 & 0.0000081 & 0.0000313 \\
\hline $\mathrm{Cl}$ & 0.0000003 & 0.0000003 & 0.0000003 & 0.0000060 & 0.0000003 & - - \\
\hline $\mathrm{Ca}$ & 0.0000021 & 0.0000021 & 0.0000021 & 0.0000010 & 0.0000021 & - \\
\hline Co & 0.0000008 & 0.0000047 & 0.0000008 & 0.0000043 & 0.0000022 & 0.0000017 \\
\hline
\end{tabular}


TABLE 4.6 (contd)

\begin{tabular}{|c|c|c|c|c|c|c|}
\hline Isotope & $\begin{array}{c}\text { Reflector } \\
\text { Stainless } \\
36-42 \\
\end{array}$ & $\begin{array}{c}\text { Outer Core } \\
\text { SFC } \\
0-6 \\
\end{array}$ & $\begin{array}{c}\text { Outer Core } \\
\text { DFC } \\
0-6 \\
\end{array}$ & $\begin{array}{c}\text { Outer Core } \\
\text { SFC } \\
6-20 \\
\end{array}$ & $\begin{array}{c}\text { Outer Core } \\
\text { DFC } \\
6-20 \\
\end{array}$ & $\begin{array}{c}\text { Axial } \\
\text { Blanket } \\
20-31\end{array}$ \\
\hline $\mathrm{C}$ & 0.0002143 & 0.0000343 & 0.0022792 & 0.0000331 & 0.0022898 & 0.0000532 \\
\hline 0 & & 0.0136985 & 0.0155311 & 0.0137649 & 0.0156113 & 0.0088222 \\
\hline $\mathrm{Na}$ & - & 0.0092311 & 0.0087208 & 0.0092111 & 0.0087495 & 0.0091804 \\
\hline $\mathrm{Si}$ & 0.0008629 & $0.0001,593$ & 0.0001840 & 0.0001574 & 0.0001817 & 0.0001926 \\
\hline $\mathrm{Al}$ & - & 0.0000038 & 0.0000057 & 0.0000041 & 0.0000062 & 0.0000029 \\
\hline $\mathrm{Mn}$ & 0.0015241 & 0.0002328 & 0.0002673 & 0.0002294 & 0.0002654 & 0.0003414 \\
\hline $\mathrm{Cr}$ & 0.0150441 & 0.0027175 & 0.0031671 & 0.0026738 & 0.0031426 & 0.0041601 \\
\hline $\mathrm{Fe}$ & 0.0531084 & 0.0130179 & 0.0111884 & 0.0128780 & 0.0110985 & 0.0147759 \\
\hline $\mathrm{Ni}$ & 0.0066621 & 0.0011875 & 0.0014329 & 0.0011697 & 0.0014240 & 0.0017666 \\
\hline $\mathrm{Cu}$ & 0.0000172 & 0.0000299 & 0.0000370 & 0.0000294 & 0.0000363 & 0.0000448 \\
\hline MO & 0.0000083 & 0.0002411 & 0.0004699 & 0.0002400 & 0.0004685 & 0.0000350 \\
\hline U5 & - & 0.0000126 & 0.0000183 & 0.0000127 & 0.0000183 & 0.0000179 \\
\hline บ8 & - & 0.0057991 & 0.0083488 & 0.0058138 & 0.0083534 & 0.0081570 \\
\hline P8 & - & 0.0000003 & 0.0000010 & 0.0000003 & 0.0000010 & - \\
\hline P9 & - & 0.0008898 & 0.0017772 & 0.0008882 & 0.0017727 & \\
\hline PO & - & 0.0001179 & 0.0002353 & 0.0001177 & 0.0002346 & \\
\hline P1 & - & 0.0000063 & 0.0000144 & 0.0000066 & 0.0000144 & - \\
\hline P2 & - & 00016 & 0.0000039 & 0.0000017 & 0.0000039 & - - \\
\hline $\mathrm{A} 1$ & - & 00103 & 0. & 106 & 0.0000216 & -- \\
\hline$P$ & -- & 00054 & 00048 & 0.0000053 & 0.0000047 & 0.0000101 \\
\hline$S$ & - & 0.0000010 & 0.0000016 & 0.0000010 & 0.0000016 & 0.0000081 \\
\hline $\mathrm{Cl}$ & - & 0.0000003 & 0.0000060 & 0.0000003 & 0.0000061 & 0.0000003 \\
\hline $\mathrm{Ca}$ & - & 0.0000021 & 0.0000010 & 0.0000021 & 0.0000010 & 0.0000021 \\
\hline Co & - & 0.0000008 & 0.0000046 & 0.0000008 & 0.0000043 & 0.0000023 \\
\hline
\end{tabular}


TABLE 4.6 (contd)

\begin{tabular}{|c|c|c|c|c|c|c|}
\hline Isotope & $\begin{array}{l}\text { Reflector } \\
\text { Iron Block } \\
\quad 31-36 \\
\end{array}$ & $\begin{array}{c}\text { Reflector } \\
\text { Stainless } \\
36-42 \\
\end{array}$ & $\begin{array}{l}\text { Radial } \\
\text { Blanket } \\
0-6 \\
\end{array}$ & $\begin{array}{c}\text { Radial } \\
\text { Blanket } \\
6-20 \\
\end{array}$ & $\begin{array}{c}\text { Axial } \\
\text { Blanket } \\
20-31 \\
\end{array}$ & $\begin{array}{l}\text { Reflector } \\
\text { Iron Block } \\
\quad 31-36 \\
\end{array}$ \\
\hline C & 0.0005875 & 0.0002143 & 0.0000331 & 0.0000323 & 0.0000323 & 0.0005923 \\
\hline 0 & - & - & 0.0224198 & 0.0225016 & 0.0224943 & - \\
\hline $\mathrm{Na}$ & - & - & 0.0042298 & 0.0042451 & 0.0042293 &.-- \\
\hline $\mathrm{Si}$ & 0.0001234 & 0.0008629 & 0.0001420 & 0.0001397 & 0.0001397 & 0.0001148 \\
\hline $\mathrm{Al}$ & - & - & 0.0000024 & 0.0000024 & 0.0000025 & - \\
\hline $\mathrm{Mn}$ & 0.0006781 & 0.0015241 & 0.0002028 & 0.0001990 & 0.0001990 & 0.0006719 \\
\hline $\mathrm{Cr}$ & 0.0020591 & 0.0150441 & 0.0023609 & 0.0023123 & 0.0023124 & 0.0019296 \\
\hline $\mathrm{Fe}$ & 0.0756250 & 0.0531084 & 0.0084347 & 0.0082639 & 0.0082641 & 0.0760459 \\
\hline $\mathrm{Ni}$ & 0.0008401 & 0.0066621 & 0.0010153 & 0.0009938 & 0.0009938 & 0.0007794 \\
\hline $\mathrm{Cu}$ & 0.0000275 & 0.0000172 & 0.0000290 & 0.0000285 & 0.0000285 & 0.0000269 \\
\hline Mo & 0.0000138 & 0.0000083 & 0.0000137 & 0.0000132 & 0.0000132 & 0.0000135 \\
\hline U5 & - & - & 0.0000288 & 0.0000290 & 0.0000289 & -- \\
\hline บ8 & - & - & 0.0132393 & 0.0132875 & 0.0132871 & - \\
\hline P8 & - & - & - & - & - & - \\
\hline P9 & - & - & - & - & - & - \\
\hline PO & - & - & - - & - & - & - \\
\hline P1 & - & - & - & - & - & - \\
\hline P2 & - & - & - & - & - - & - \\
\hline $\mathrm{A} 1$ & - & - & - & - & - & - - \\
\hline$P$ & 0.0000235 & - & 0.0000052 & 0.0000051 & 0.0000051 & 0.0000233 \\
\hline$S$ & 0.0000313 & - & 0.0000011 & 0.0000011 & 0.0000011 & 0.0000314 \\
\hline $\mathrm{Cl}$ & - & - & 0.0000001 & 0.0000001 & 0.0000001 & $\ldots$ \\
\hline $\mathrm{Ca}$ & - & - & 0.0000010 & 0.0000010 & 0.0000010 & - \\
\hline Co & 0.0000017 & - & 0.0000017 & 0.0000017 & 0.0000017 & 0.0000012 \\
\hline
\end{tabular}


TABLE 4.6 (contd)

\begin{tabular}{|c|c|c|c|c|c|c|}
\hline Isotope & $\begin{array}{c}\text { Reflector } \\
\text { Stainless } \\
\quad 36-42 \\
\end{array}$ & $\begin{array}{c}\text { Reflector } \\
\text { Reflector } \\
0-36 \\
\end{array}$ & $\begin{array}{c}\text { Central } \\
\text { Blanket } \\
(\mathrm{SFC}+\mathrm{DFC}) \\
0-6 \\
\end{array}$ & $\begin{array}{c}\text { Inner Core } \\
(\mathrm{SFC}+\mathrm{DFC}) \\
6-20 \\
\end{array}$ & $\begin{array}{c}\text { Outer Core } \\
(\mathrm{SFC}+\mathrm{DFC}) \\
0-6 \\
\end{array}$ & $\begin{array}{r}\text { Outer Core } \\
(\mathrm{SFC}+\mathrm{DFC}) \\
6-20\end{array}$ \\
\hline C & 0.0002143 & 0.0002506 & 0.0000337 & 0.0009254 & 0.0009380 & 0.0009416 \\
\hline 0 & -- & - & 0.0136978 & 0.0144887 & 0.0144375 & 0.0145095 \\
\hline $\mathrm{Na}$ & - & -- & 0.0091902 & 0.0090633 & 0.0090264 & 0.0090259 \\
\hline $\mathrm{Si}$ & 0.0008629 & 0.0008751 & 0.0001459 & 0.0001670 & 0.0001693 & 0.0001672 \\
\hline $\mathrm{Al}$ & - & -- & 0.0000029 & 0.0000049 & 0.0000046 & 0.0000049 \\
\hline Mn & 0.0015241 & 0.0013948 & 0.0002061 & 0.0002436 & 0.0002467 & 0.0002439 \\
\hline $\mathrm{Cr}$ & 0.0150441 & 0.0153536 & 0.0024129 & 0.0028588 & 0.0028987 & 0.0028628 \\
\hline $\mathrm{Fe}$ & 0.0531084 & 0.0545599 & 0.0119522 & 0.0121688 & 0.0122826 & 0.0121628 \\
\hline $\mathrm{Ni}$ & 0.0066621 & 0.0066686 & 0.0010480 & 0.0012702 & 0.0012864 & 0.0012722 \\
\hline $\mathrm{Cu}$ & 0.0000172 & 0.0000396 & 0.0000296 & 0.0000322 & 0.0000328 & 0.0000322 \\
\hline Mo & 0.0000083 & 0.0000366 & 0.0000139 & 0.0003282 & 0.0003333 & 0.0003320 \\
\hline U5 & - & -- & 0.0000178 & 0.0000149 & 0.0000149 & 0.0000149 \\
\hline U8 & - & -- & 0.0081363 & 0.0068136 & 0.0068261 & 0.0068368 \\
\hline P8 & - & - & -- & 0.0000007 & 0.0000006 & 0.0000006 \\
\hline P9 & - & - & - & 0.0012308 & 0.0012471 & 0.0012444 \\
\hline PO & - & - & - - & 0.0001627 & 0.0001652 & 0.0001648 \\
\hline P1 & - & - & - & 0.0000100 & 0.0000096 & 0.0000097 \\
\hline P2 & - & - - & - - & 0.0000026 & 0.0000025 & 0.0000026 \\
\hline $\mathrm{A} 1$ & - - & - - & - - & 0.0000149 & 0.0000149 & 0.0000151 \\
\hline$P$ & $-\cdots$ & 0.0000457 & 0.0000051 & 0.0000051 & 0.0000052 & 0.0000051 \\
\hline$S$ & - & 0.0000352 & 0.0000011 & 0.0000012 & 0.0000012 & 0.0000012 \\
\hline $\mathrm{Cl}$ & - & -- & 0.0000003 & 0.0000026 & 0.0000026 & 0.0000026 \\
\hline $\mathrm{Ca}$ & - & - & 0.0000021 & 0.0000017 & 0.0000016 & 0.0000016 \\
\hline Co & - & 0.0000009 & 0.0000024 & 0.0000022 & 0.0000023 & 0.0000022 \\
\hline
\end{tabular}


TABLE 4.6 (contd)

\begin{tabular}{|c|c|c|c|}
\hline Isotope & $\begin{array}{c}\text { Empty } \\
\text { Matrix } \\
0-60\end{array}$ & $\begin{array}{c}\text { Control } \\
\text { Position } \\
0-20 \\
\end{array}$ & $\begin{array}{c}\text { Control } \\
\text { Position } \\
20-31 \\
\end{array}$ \\
\hline C & 0.0000188 & 0.0000314 & 0.0000369 \\
\hline 0 & -- & 0.0000013 & 0.0000013 \\
\hline $\mathrm{Na}$ & - & 0.0182024 & 0.0182389 \\
\hline $\mathrm{Si}$ & 0.0000683 & 0.0001670 & 0.0001667 \\
\hline $\mathrm{A} 1$ & -- & 0.0000045 & 0.0000049 \\
\hline Mn & 0.0001059 & 0.0002460 & 0.0002462 \\
\hline $\mathrm{Cr}$ & 0.0011891 & 0.0029889 & 0.0029856 \\
\hline $\mathrm{Fe}$ & 0.0042791 & 0.0105526 & 0.0106624 \\
\hline $\mathrm{Ni}$ & 0.0004802 & 0.0013379 & 0.0013366 \\
\hline $\mathrm{Cu}$ & 0.0000172 & 0.0000361 & 0.0000358 \\
\hline Mo & 0.0000083 & 0.0000177 & 0.0000177 \\
\hline U5 & - - & - & - - \\
\hline U8 & -- & - - & - \\
\hline P8 & - & - - & - \\
\hline P9 & - - & - & - \\
\hline PO & - & - - & - \\
\hline P1 & - & - - & - \\
\hline P2 & - - & - & - \\
\hline A1 & - - & - - & - - \\
\hline$P$ & 0.0000028 & 0.0000041 & 0.0000040 \\
\hline$S$ & 0.0000007 & 0.0000013 & 0.0000012 \\
\hline $\mathrm{Cl}$ & - & 0.0000006 & 0.0000006 \\
\hline $\mathrm{Ca}$ & - & 0.0000041 & 0.0000042 \\
\hline Co & - & 0.0000039 & 0.0000040 \\
\hline
\end{tabular}


TABLE 4.7 ZPPR Assembly 17C: Atom Densities by Zone

\begin{tabular}{|c|c|c|c|c|c|c|}
\hline Isotope & $\begin{array}{c}\text { Empty } \\
\text { Matrix } \\
0-36 \\
\end{array}$ & $\begin{array}{c}\text { Radial } \\
\text { Reflector } \\
0-36 \\
\end{array}$ & $\begin{array}{c}\text { Axial } \\
\text { Reflector } \\
36-42 \\
\end{array}$ & $\begin{array}{l}\text { Radial } \\
\text { Blanket } \\
31-36 \\
\end{array}$ & $\begin{array}{l}\text { Radial } \\
\text { Blanket } \\
20-31 \\
\end{array}$ & $\begin{array}{c}\text { Radial } \\
\text { Blanket } \\
6-20 \\
\end{array}$ \\
\hline BO & - & - & - & - - & - - & - \\
\hline B1 & -- & - & - - & - & - & - \\
\hline C & 0.0000188 & 0.0002440 & 0.0002143 & 0.0005923 & 0.0000323 & 0.0000323 \\
\hline 0 & - & -- & - & -- & 0.0224943 & 0.0225016 \\
\hline $\mathrm{Na}$ & - & - & - & - & 0.0042293 & 0.0042451 \\
\hline$S i$ & 0.0000683 & 0.0009017 & 0.0008629 & 0.0001148 & 0.0001397 & 0.0001397 \\
\hline $\mathrm{Al}$ & -- & - & -- & - & 0.0000025 & 0.0000024 \\
\hline Mn & 0.0001059 & 0.0014353 & 0.0015241 & 0.0006719 & 0.0001990 & 0.0001990 \\
\hline $\mathrm{Cr}$ & 0.0011891 & 0.0157477 & 0.0150441 & 0.0019296 & 0.0023124 & 0.0023123 \\
\hline $\mathrm{Fe}$ & 0.0042791 & 0.0556680 & 0.0531083 & 0.0760459 & 0.0082641 & 0.0082639 \\
\hline $\mathrm{Ni}$ & 0.0004802 & 0.0068483 & 0.0066621 & 0.0007794 & 0.0009938 & 0.0009938 \\
\hline $\mathrm{Cu}$ & 0.0000172 & 0.0000379 & 0.0000172 & 0.0000269 & 0.0000285 & 0.0000285 \\
\hline Mo & 0.0000083 & 0.0000356 & 0.0000083 & 0.0000135 & 0.0000132 & 0.0000132 \\
\hline U5 & - & -- & - & - & 0.0000289 & 0.0000290 \\
\hline บ8 & - & - & - - & - & 0.0132871 & 0.0132875 \\
\hline $\mathrm{P} 8$ & - - & - - & - & -- & - & $\ldots$ \\
\hline P9 & - - & - - & - & - - & - - & \\
\hline PO & -- & - - & -- & - & -- & - \\
\hline P1 & - & - & - - & - & - - & - \\
\hline $\mathrm{P} 2$ & - & - - & - - & - - & - - & - \\
\hline A1 & - & - & - & - & - & -- \\
\hline $\mathrm{P}$ & 0.0000028 & 0.0000472 & 0.0000533 & 0.0000233 & 0.0000051 & 0.0000051 \\
\hline$S$ & 0.0000007 & 0.0000363 & 0.0000252 & 0.0000314 & 0.0000011 & 0.0000011 \\
\hline $\mathrm{Cl}$ & - - & - & - - & - & 0.0000001 & 0.0000001 \\
\hline $\mathrm{Ca}$ & - - & - & - & -- & 0.0000010 & 0.0000010 \\
\hline Co & - - & 0.0000007 & - & 0.0000012 & 0.0000017 & 0.0000017 \\
\hline
\end{tabular}


TABLE 4.7 (cont d)

\begin{tabular}{|c|c|c|c|c|c|c|}
\hline Isotope & $\begin{array}{c}\text { Radial } \\
\text { Blanket } \\
0-6 \\
\end{array}$ & $\begin{array}{c}\text { Axial Refl } \\
\text { Outer Core } \\
31-36 \\
\end{array}$ & $\begin{array}{c}\text { Axial Blkt } \\
\text { Outer Core } \\
20-31 \\
\end{array}$ & $\begin{array}{c}\text { Outer Core } \\
6-20 \\
\end{array}$ & $\begin{array}{c}\text { Outer Core } \\
0-6 \\
\end{array}$ & $\begin{array}{c}\text { Axial Refl } \\
\text { Inner Core } \\
31-36 \\
\end{array}$ \\
\hline BO & - & - & - & - & - & - \\
\hline B1 & - & -- & - - & - & - & - \\
\hline C & 0.0000333 & 0.0005874 & 0.0000532 & 0.0012314 & 0.0012263 & 0.0005884 \\
\hline 0 & 0.0223887 & -- & 0.0088214 & 0.0147521 & 0.0146783 & - \\
\hline $\mathrm{Na}$ & 0.0042238 & - & 0.0091799 & 0.0089549 & 0.0089446 & - \\
\hline Si & 0.0001428 & 0.0001236 & 0.0001928 & 0.0001703 & 0.0001724 & 0.0001232 \\
\hline Al & 0.0000024 & -- & 0.0000029 & 0.0000052 & 0.0000049 & - \\
\hline Mn & 0.0002038 & 0.0006777 & 0.0003411 & 0.0002486 & 0.0002513 & 0.0006779 \\
\hline $\mathrm{Cr}$ & 0.0023727 & 0.0020570 & 0.0041582 & 0.0029237 & 0.0029578 & 0.0020511 \\
\hline $\mathrm{Fe}$ & 0.0084772 & 0.0756063 & 0.0147653 & 0.0119410 & 0.0120566 & 0.0757124 \\
\hline $\mathrm{Ni}$ & 0.0010206 & 0.0008422 & 0.0017683 & 0.0013052 & 0.0013186 & 0.0008390 \\
\hline $\mathrm{Cu}$ & 0.0000292 & 0.0000281 & 0.0000454 & 0.0000331 & 0.0000337 & 0.0000283 \\
\hline Mo & 0.0000138 & 0.0000141 & 0.0000353 & 0.0003610 & 0.0003624 & 0.0000142 \\
\hline U5 & 0.0000288 & - & 0.0000179 & 0.0000156 & 0.0000156 & - \\
\hline U8 & 0.0132208 & - & 0.0081563 & 0.0071563 & 0.0071450 & - - \\
\hline P8 & - & - & - & 0.0000007 & 0.0000007 & - - \\
\hline P9 & - - & - & - - & 0.0013565 & 0.0013596 & - \\
\hline PO & - - & - & - - & 0.0001796 & 0.0001801 & - \\
\hline P1 & - - & - & - & 0.0000108 & 0.0000106 & \\
\hline P2 & - - & - & - - & 0.0000028 & 0.0000028 & \\
\hline A1 & - & - & - - & 0.0000164 & 0.0000162 & - \\
\hline $\mathrm{P}$ & 0.0000052 & 0.0000234 & 0.0000100 & 0.0000050 & 0.0000051 & 0.0000234 \\
\hline S & 0.0000011 & 0.0000313 & 0.0000082 & 0.0000013 & 0.0000013 & 0.0000314 \\
\hline $\mathrm{Cl}$ & 0.0000001 & - & 0.0000003 & 0.0000034 & 0.0000033 & - \\
\hline $\mathrm{Ca}$ & 0.0000010 & - - & 0.0000021 & 0.0000015 & 0.0000015 & - \\
\hline Co & 0.0000018 & 0.0000022 & 0.0000027 & 0.0000027 & 0.0000028 & 0.0000022 \\
\hline
\end{tabular}


TABLE 4.7 (contd)

\begin{tabular}{|c|c|c|c|c|c|c|}
\hline Isotope & $\begin{array}{c}\text { Axial Blkt } \\
\text { Inner Core } \\
20-31 \\
\end{array}$ & $\begin{array}{c}\text { Inner Core } \\
6-20 \\
\end{array}$ & $\begin{array}{c}\text { Inner Core } \\
0-6 \\
\end{array}$ & $\begin{array}{c}\text { Axial Refl } \\
\text { Outer Core } \\
\text { SCF } \\
31-36 \\
\end{array}$ & $\begin{array}{c}\text { Axial Blkt } \\
\text { Outer Core } \\
\text { SCF } \\
20-31 \\
\end{array}$ & $\begin{array}{c}\text { Outer Core } \\
\text { SCF } \\
6-20 \\
\end{array}$ \\
\hline BO & - - & - & - & - - & - & - \\
\hline B1 & - & - & - - & - - & - & - \\
\hline $\mathrm{C}$ & 0.0000532 & 0.0012105 & 0.0000338 & 0.0005855 & 0.0000532 & 0.0000331 \\
\hline 0 & 0.0088212 & 0.0147284 & 0.0136973 & -- & 0.0088213 & 0.0137650 \\
\hline $\mathrm{Na}$ & 0.0092079 & 0.0089966 & 0.0091901 & - & 0.0091325 & 0.0091844 \\
\hline Si & 0.0001928 & 0.0001701 & 0.0001468 & 0.0001260 & 0.0001918 & 0.0001574 \\
\hline Al & 0.0000029 & 0.0000051 & 0.0000029 & - & 0.0000029 & 0.0000041 \\
\hline Mn & 0.0003412 & 0.0002484 & 0.0002065 & 0.0006816 & 0.0003421 & 0.0002293 \\
\hline $\mathrm{Cr}$ & 0.0041587 & 0.0029197 & 0.0024191 & 0.0021181 & 0.0041628 & 0.0026730 \\
\hline $\mathrm{Fr}$ & 0.0147671 & 0.0119542 & 0.0119711 & 0.0754880 & 0.0147973 & 0.0128752 \\
\hline $\mathrm{Ni}$ & 0.0017686 & 0.0013032 & 0.0010530 & 0.0008569 & 0.0017595 & 0.0011693 \\
\hline $\mathrm{Cu}$ & 0.0000454 & 0.0000331 & 0.0000303 & 0.0000256 & 0.0000432 & 0.0000294 \\
\hline Mo & 0.0000353 & 0.0003567 & 0.0000143 & 0.0000128 & 0.0000341 & 0.0002398 \\
\hline U5 & 0.0000179 & 0.0000156 & 0.0000178 & - & 0.0000179 & 0.0000127 \\
\hline U8 & 0.0081561 & 0.0071223 & 0.0081361 & - & 0.0081562 & 0.0058134 \\
\hline P8 & - & 0.0000007 & -- & - & -- & 0.0000004 \\
\hline P9 & $-\cdots$ & 0.0013416 & - & - & - & 0.0008879 \\
\hline PO & - & 0.0001774 & - - & - & -- & 0.0001177 \\
\hline P1 & - & 0.0000108 & -- & - - & - & 0.0000066 \\
\hline P2 & -- & 0.0000028 & - & - - & -- & 0.0000017 \\
\hline A1 & - & 0.0000162 & - & - - & -- & 0.0000107 \\
\hline $\mathrm{P}$ & 0.0000100 & 0.0000050 & 0.0000051 & 0.0000238 & 0.0000103 & 0.0000053 \\
\hline$S$ & 0.0000082 & 0.0000013 & 0.0000012 & 0.0000312 & 0.0000080 & 0.0000010 \\
\hline $\mathrm{Cl}$ & 0.0000003 & 0.0000033 & 0.0000003 & -- & 0.0000003 & 0.0000003 \\
\hline $\mathrm{Ca}$ & 0.0000021 & 0.0000015 & 0.0000021 & - & 0.0000021 & 0.0000021 \\
\hline Co & 0.0000027 & 0.0000027 & 0.0000029 & - & 0.0000008 & 0.0000008 \\
\hline
\end{tabular}


Table 4.7 (contd)

\begin{tabular}{|c|c|c|c|c|c|c|}
\hline Isotope & $\begin{array}{c}\text { Outer Core } \\
\text { SCF } \\
0-6 \\
\end{array}$ & $\begin{array}{c}\text { Axial Refl } \\
\text { Outer Core } \\
\text { DCF } \\
31-36 \\
\end{array}$ & $\begin{array}{c}\text { Axial Blkt } \\
\text { Outer Core } \\
\text { DCF } \\
20-31 \\
\end{array}$ & $\begin{array}{c}\text { Outer Core } \\
\text { DCF } \\
6-20 \\
\end{array}$ & $\begin{array}{c}\text { Outer Core } \\
\text { DCE } \\
0-6 \\
\end{array}$ & $\begin{array}{c}\text { Axial Refl } \\
\text { Inner Core } \\
\text { SCF } \\
31-36 \\
\end{array}$ \\
\hline BO & - - & - & $\therefore-$ & - & - & - - \\
\hline B1 & -- & -- & - & - & -- & \\
\hline C & 0.0000343 & 0.0005891 & 0.0000532 & 0.0022955 & 0.0022849 & 0.0005885 \\
\hline 0 & 0.0136986 & -- & 0.0088213 & 0.0156284 & 0.0155480 & - \\
\hline $\mathrm{Na}$ & 0.0092043 & - & 0.0092218 & 0.0087510 & 0.0087138 & - \\
\hline Si & 0.0001592 & 0.0001216 & 0.0001937 & 0.0001818 & 0.0001842 & 0.0001232 \\
\hline $\mathrm{Al}$ & 0.0000038 & - & 0.0000029 & 0.0000063 & 0.0000058 & - - \\
\hline $\mathrm{Mn}$ & 0.0002328 & 0.0006742 & 0.0003403 & 0.0002657 & 0.0002677 & 0.0006808 \\
\hline $\mathrm{Cr}$ & 0.0027171 & 0.0020027 & 0.0041540 & 0.0031463 & 0.0031715 & 0.0020771 \\
\hline $\mathrm{Fe}$ & 0.0130166 & 0.0757097 & 0.0147367 & 0.0111112 & 0.0112039 & 0.0758039 \\
\hline $\mathrm{Ni}$ & 0.0011873 & 0.0008291 & 0.0017761 & 0.0014258 & 0.0014351 & 0.0008375 \\
\hline $\mathrm{Cr}$ & 0.0000299 & 0.0000303 & 0.0000473 & 0.0000363 & 0.0000370 & 0.0000260 \\
\hline Mo & 0.0002408 & 0.0000153 & 0.0000363 & 0.0004686 & 0.0004704 & 0.0000130 \\
\hline U5 & 0.0000126 & -- & 0.0000179 & 0.0000183 & 0.0000183 & -- \\
\hline U8 & 0.0057979 & - & 0.0081562 & 0.0083486 & 0.0083410 & \\
\hline P8 & 0.0000003 & - & -- & 0.0000010 & 0.0000009 & \\
\hline P9 & 0.0008892 & - & - & 0.0017726 & 0.0017773 & - \\
\hline PO & 0.0001178 & - & -- & 0.0002346 & 0.0002353 & -- \\
\hline P1 & 0.0000063 & - & - & 0.0000145 & 0.0000143 & -- \\
\hline P2 & 0.0000016 & - & - & 0.0000038 & 0.0000038 & -- \\
\hline A1 & 0.0000103 & - & - & 0.0000214 & 0.0000214 & - \\
\hline $\mathrm{P}$ & 0.0000054 & 0.0000230 & 0.0000098 & 0.0000047 & 0.0000048 & 0.0000238 \\
\hline$S$ & 0.0000010 & 0.0000315 & 0.0000083 & 0.0000016 & 0.0000016 & 0.0000312 \\
\hline $\mathrm{Cl}$ & 0.0000003 & - & 0.0000003 & 0.0000062 & 0.0000061 & - \\
\hline $\mathrm{Ca}$ & 0.0000021 & - & 0.0000021 & 0.0000010 & 0.0000010 & - \\
\hline Co & 0.0000008 & 0.0000042 & 0.0000044 & 0.0000043 & 0.0000046 & - \\
\hline
\end{tabular}


TABLE 4.7 (contd)

\begin{tabular}{|c|c|c|c|c|c|c|}
\hline Isotope & $\begin{array}{c}\text { Axial Blkt } \\
\text { Inner Core } \\
\text { SCF } \\
20-31 \\
\end{array}$ & $\begin{array}{c}\text { Inner Core } \\
\text { SCF } \\
6-20 \\
\end{array}$ & $\begin{array}{c}\text { Inner Core } \\
\text { SCF } \\
0-6 \\
\end{array}$ & $\begin{array}{c}\text { Axial Refl } \\
\text { Inner Core } \\
\text { DCE } \\
31-36 \\
\end{array}$ & $\begin{array}{c}\text { Axial Blkt } \\
\text { Inner Core } \\
\text { DCF } \\
20-31 \\
\end{array}$ & $\begin{array}{c}\text { Inner Core } \\
\text { DCF } \\
6-20 \\
\end{array}$ \\
\hline $\mathrm{BO}$ & -- & - & - - & - & - & - \\
\hline B.1 & -- & - & - & - - & -- & - \\
\hline C & 0.0000532 & 0.0000331 & 0.0000333 & 0.0005882 & 0.0000532 & 0.0022897 \\
\hline 0 & 0.0088213 & 0.0137650 & 0.0136987 & -- & 0.0088213 & 0.0156118 \\
\hline $\mathrm{Na}$ & 0.0092050 & 0.0092702 & 0.0091887 & - & 0.0092107 & 0.0087458 \\
\hline $\mathrm{Si}$ & 0.0001919 & 0.0001569 & 0.0001434 & 0.0001232 & 0.0001937 & 0.0001821 \\
\hline Al & 0.0000029 & 0.0000040 & 0.0000029 & -- & 0.0000029 & 0.0000062 \\
\hline$M n$ & 0.0003422 & 0.0002281 & 0.0002047 & 0.0006753 & 0.0003402 & 0.0002669 \\
\hline $\mathrm{Cr}$ & 0.0041646 & 0.0026601 & 0.0023942 & 0.0020274 & 0.0041534 & 0.0031576 \\
\hline $\mathrm{Fe}$ & 0.0148035 & 0.0128309 & 0.0118954 & 0.0756294 & 0.0147340 & 0.0111507 \\
\hline $\mathrm{Ni}$ & 0.0017604 & 0.0011629 & 0.0010325 & 0.0008404 & 0.0017760 & 0.0014319 \\
\hline $\mathrm{Cu}$ & 0.0000432 & 0.0000294 & 0.0000274 & 0.0000304 & 0.0000473 & 0.0000365 \\
\hline Mo & 0.0000341 & 0.0002394 & 0.0000129 & 0.0000154 & 0.0000364 & 0.0004643 \\
\hline U5 & 0.0000179 & 000127 & 00178 & - - & 0.0000179 & 0.0000182 \\
\hline U8 & 0.0081562 & 0 & 0.0081368 & - & 0.0081562 & 0.0082988 \\
\hline P8 & - & 000005 & - & - & - & 0.0000009 \\
\hline P9 & - - & 0.0008874 & - & - & - & 0.0017580 \\
\hline PO & - - & 0.0001175 & - - & - & - - & 0.0002323 \\
\hline P1 & - - & 0.0000074 & - - & - & - & 0.0000139 \\
\hline P2 & - - & 0.0000019 & - - & - & - - & 0.0000036 \\
\hline A1 & -- & 0.0000109 & -- & - & -- & 0.0000210 \\
\hline$P$ & 0.0000103 & 0.0000053 & 0.0000053 & 0.0000231 & 0.0000098 & 0.0000047 \\
\hline$S$ & 0.0000080 & 0.0000010 & 0.0000010 & 0.0000315 & 0.0000083 & 0.0000016 \\
\hline $\mathrm{Cl}$ & 0.0000003 & 0.0000003 & 0.0000003 & - & 0.0000003 & 0.0000060 \\
\hline $\mathrm{Ca}$ & 0.0000021 & 0.0000021 & 0.0000021 & - - & 0.0000021 & 0.0000010 \\
\hline $\mathrm{Co}$ & 0.0000008 & 0.0000008 & 0.0000008 & 0.0000043 & 0.0000045 & 0.0000044 \\
\hline
\end{tabular}


TABLE 4.7 (contd)

\begin{tabular}{|c|c|c|c|c|c|c|}
\hline Isotope & $\begin{array}{c}\text { Inner Blkt } \\
0-6 \\
\end{array}$ & $\begin{array}{c}\text { Control } \\
\text { Position } \\
31-36 \\
\end{array}$ & $\begin{array}{c}\text { Control } \\
\text { Position } \\
20-31 \\
\end{array}$ & $\begin{array}{c}\text { Control } \\
\text { Position } \\
6-20 \\
\end{array}$ & $\begin{array}{c}\text { Control } \\
\text { Position } \\
0-6 \\
\end{array}$ & $\begin{array}{c}\text { Bank } \\
\text { Control } \\
\text { Rod } \\
31-36 \\
\end{array}$ \\
\hline BO & - & - & - & - - & - & - - \\
\hline B1 & - & - - & - & - - & -- & - \\
\hline $\mathrm{C}$ & 0.0000342 & 0.0000443 & 0.0000309 & 0.0000309 & 0.0000318 & 0.0000508 \\
\hline 0 & 0.0136964 & 0.0000013 & 0.0000013 & 0.0000013 & 0.0000013 & 0.0000013 \\
\hline $\mathrm{Na}$ & 0.0091915 & 0.0179925 & 0.0184405 & 0.0183970 & 0.0180431 & 0.0180018 \\
\hline Si & 0.0001499 & 0.0001688 & 0.0001649 & 0.0001649 & 0.0001688 & 0.0001863 \\
\hline $\mathrm{AI}$ & 0.0000029 & 0.0000048 & 0.0000049 & 0.0000048 & 0.0000043 & 0.0000056 \\
\hline $\mathrm{Mn}$ & 0.0002082 & 0.0002486 & 0.0002443 & 0.0002442 & 0.0002474 & 0.0002517 \\
\hline $\mathrm{Cr}$ & 0.0024420 & 0.0030099 & 0.0029657 & 0.0029644 & 0.0030089 & 0.0030170 \\
\hline $\mathrm{Fe}$ & 0.0120407 & 0.0108979 & 0.0104697 & 0.0104649 & 0.0106243 & 0.0109629 \\
\hline $\mathrm{Ni}$ & 0.0010718 & 0.0013470 & 0.0013281 & 0.0013274 & 0.0013465 & 0.0013664 \\
\hline $\mathrm{Cu}$ & 0.0000329 & 0.0000365 & 0.0000353 & 0.0000353 & 0.0000367 & 0.0000364 \\
\hline Mo & 0.0000155 & 0.0000181 & 0.0000174 & 0.0000174 & 0.0000180 & 0.0000160 \\
\hline U5 & 0.0000178 & - & - & - & - & - \\
\hline U8 & 0.0081355 & - & - & -- & -- & - \\
\hline P8 & -- & -- & - & - & -- & - - \\
\hline P9 & - & - & - - & - - & - & - - \\
\hline PO & - - & - & - - & - - & - & - \\
\hline P1 & - & - & - & -- & -- & - \\
\hline$P 2$ & - & -- & - & - & - & - - \\
\hline A1 & -- & - & - & -- & - & -- \\
\hline $\mathrm{P}$ & 0.0000049 & 0.0000041 & 0.0000040 & 0.0000040 & 0.0000041 & 0.0000057 \\
\hline S & 0.0000013 & 0.0000013 & 0.0000012 & 0.0000012 & 0.0000013 & 0.0000013 \\
\hline $\mathrm{Cl}$ & 0.0000003 & 0.0000006 & 0.0000006 & 0.0000006 & 0.0000006 & 0.0000006 \\
\hline $\mathrm{Ca}$ & 0.0000021 & 0.0000041 & 0.0000042 & 0.0000042 & 0.0000041 & 0.0000041 \\
\hline Co & 0.0000048 & 0.0000043 & 0.0000037 & 0.0000037 & 0.0000040 & 0.0000059 \\
\hline
\end{tabular}


TABLE 4.7 (contd)

\begin{tabular}{|c|c|c|c|c|c|c|}
\hline Isotope & $\begin{array}{c}\text { Bank } \\
\text { Control } \\
\text { Rod } \\
20-31 \\
\end{array}$ & $\begin{array}{c}\text { Bank } \\
\text { Control } \\
\text { Rod } \\
6-20 \\
\end{array}$ & $\begin{array}{c}\text { Bank } \\
\text { Control } \\
\text { Rod } \\
0-6 \\
\end{array}$ & $\begin{array}{c}\text { Central } \\
\text { Control } \\
\text { Rod } \\
31-36 \\
\end{array}$ & $\begin{array}{c}\text { Central } \\
\text { Control } \\
\text { Rod } \\
20-31 \\
\end{array}$ & $\begin{array}{c}\text { Central } \\
\text { Control } \\
\text { Rod } \\
6-20 \\
\end{array}$ \\
\hline BO & 0.0160061 & 0.0151769 & 0.0158703 & - & 0.0160061 & 0.0157321 \\
\hline B1. & 0.0649083 & 0.0615467 & 0.0643535 & - & 0.0649083 & 0.0637966 \\
\hline $\mathrm{C}$ & 0.0208421 & 0.0197666 & 0.0203715 & 0.0000508 & 0.0208421 & 0.0204374 \\
\hline 0 & 0.0000803 & 0.0000534 & 0.0001353 & 0.0000013 & 0.0000803 & 0.0000825 \\
\hline $\mathrm{Na}$ & - & -- & -- & 0.0180018 & - & -- \\
\hline Si & 0.0002047 & 0.0002235 & 0.0002562 & 0.0001863 & 0.0002047 & 0.0002188 \\
\hline AI & 0.0000010 & 0.0000028 & 0.0000021 & 0.0000056 & 0.0000010 & 0.0000020 \\
\hline Mn & 0.0001928 & 0.0002204 & 0.0002066 & 0.0002517 & 0.0001928 & 0.0002028 \\
\hline $\mathrm{Cr}$ & 0.0022421 & 0.0026105 & 0.0024203 & 0.0030170 & 0.0022421 & 0.0023755 \\
\hline $\mathrm{Fe}$ & 0.0081410 & 0.0093681 & 0.0087156 & 0.0109629 & 0.0081410 & 0.0085838 \\
\hline $\mathrm{Ni}$ & 0.0009734 & 0.0011621 & 0.0010609 & 0.0013664 & 0.0009734 & 0.0010417 \\
\hline $\mathrm{Cu}$ & 0.0000307 & 0.0000325 & 0.0000325 & 0.0000364 & 0.0000307 & 0.0000314 \\
\hline Mo & 0.0000155 & 0.0000162 & 0.0000162 & 0.0000160 & 0.0000155 & 0.0000158 \\
\hline U5 & - & - & - & - & -- & -- \\
\hline U8 & - & - & - - & - - & - - & - \\
\hline P8 & - & - & - & - & - & - \\
\hline P9 & - & - & - & - & - - & - \\
\hline PO & - & - & - - & - & - - & $\ldots$ \\
\hline P1 & - & - & - - & -- & - & - - \\
\hline P2 & - - & - & - & - - & - - & - - \\
\hline A1 & - & - & - & - - & - - & - \\
\hline $\mathrm{P}$ & 0.0000040 & 0.0000040 & 0.0000041 & 0.0000057 & 0.0000040 & 0.0000040 \\
\hline$S$ & 0.0000012 & 0.0000012 & 0.0000013 & 0.0000013 & 0.0000012 & 0.0000012 \\
\hline $\mathrm{Cl}$ & -- & -- & - & 0.0000006 & -- & -- \\
\hline $\mathrm{Ca}$ & - & - & - & 0.0000041 & - & - \\
\hline Co & 0.0000037 & 0.0000037 & 0.0000040 & 0.0000059 & 0.0000037 & 0.0000037 \\
\hline
\end{tabular}


Table 4.7 (contd)

\begin{tabular}{|c|c|}
\hline Isotope & $\begin{array}{c}\text { Central } \\
\text { Control } \\
\text { Rod } \\
0-6 \\
\end{array}$ \\
\hline $\mathrm{BO}$ & 0.0158703 \\
\hline B1 & 0.0643535 \\
\hline $\mathrm{C}$ & 0.0203715 \\
\hline 0 & 0.0001353 \\
\hline $\mathrm{Na}$ & -- \\
\hline $\mathrm{Si}$ & 0.0002562 \\
\hline Al & 0.0000021 \\
\hline $\mathrm{Mn}$ & 0.0002066 \\
\hline $\mathrm{Cr}$ & 0.0024203 \\
\hline $\mathrm{Fe}$ & 0.0087156 \\
\hline $\mathrm{Ni}$ & 0.0010609 \\
\hline $\mathrm{Cu}$ & 0.0000325 \\
\hline Mo & 0.0000162 \\
\hline U5 & -- \\
\hline U8 & -- \\
\hline P8 & - \\
\hline PQ & -- \\
\hline PO & -- \\
\hline $\mathrm{P} 1$ & - \\
\hline $\mathrm{P} 2$ & - \\
\hline$A 1$ & - \\
\hline $\mathrm{P}$ & 0.0000041 \\
\hline $\mathrm{S}$ & 0.0000013 \\
\hline $\mathrm{Cl}$ & - \\
\hline $\mathrm{Ca}$ & -- \\
\hline Co & 0.0000040 \\
\hline
\end{tabular}


Fig. 4.1 Slab Model used to Collapse

Cross Sections for ZPPR-17A

\begin{tabular}{ccccc}
\hline $\begin{array}{c}\text { Axial } \\
\text { Height, cm }\end{array}$ & & \multicolumn{1}{c}{ Zone } & $\begin{array}{c}\text { Cell Cross } \\
\text { Sections }\end{array}$ \\
15.31 & & 1. Internal Blanket & IBKN \\
27.16 & 2. Inner Core Single Column & SCFN \\
39.01 & 3. Inner Core Double Column & DCFN \\
50.86 & 4. Inner Core Single Column & SCFN \\
78.82 & 5. Axial Blanket & ABKN \\
91.09 & 6. Iron Axial Reflector & ARI \\
106.33 & 7. Steel Axial Reflector & ARS \\
\hline
\end{tabular}




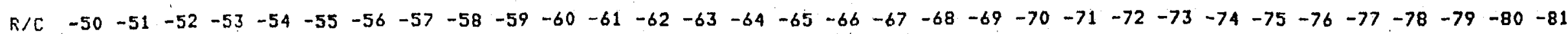

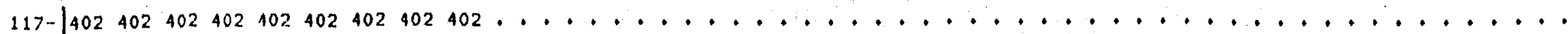

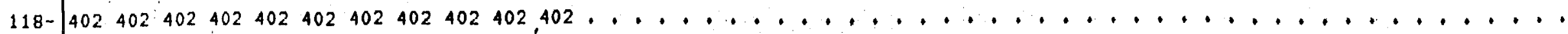

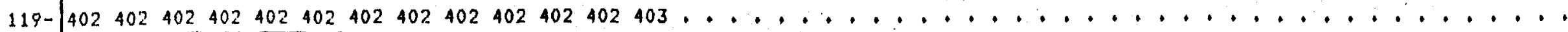

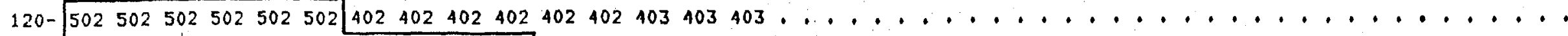

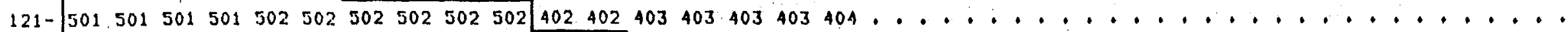

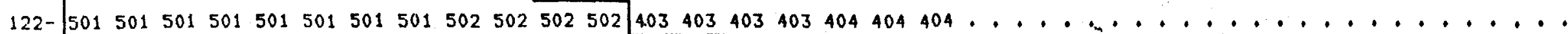

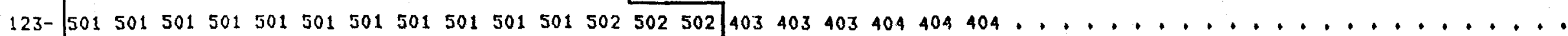

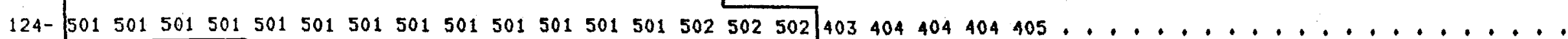

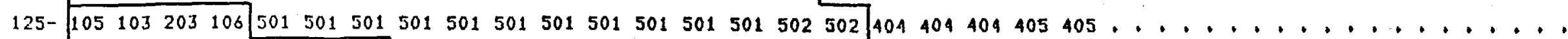

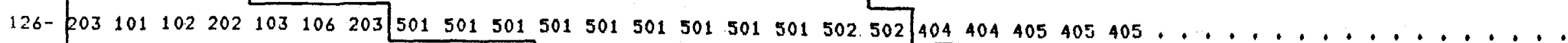

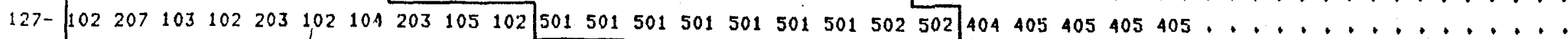

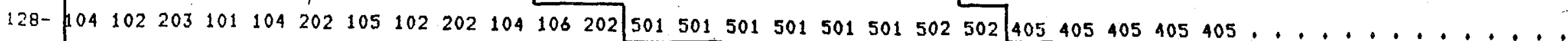

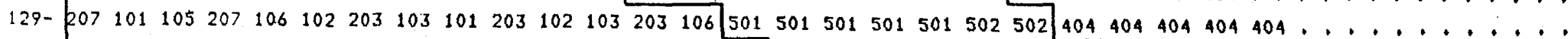

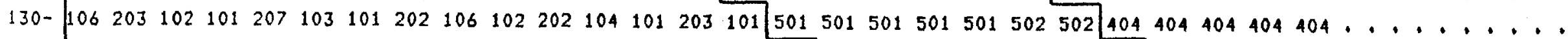

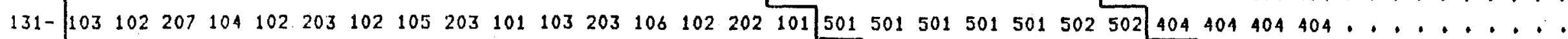

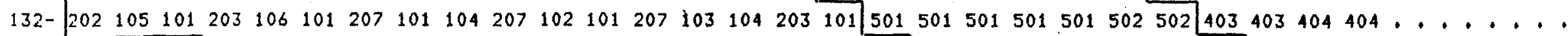

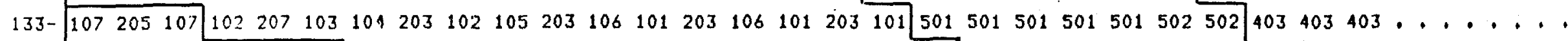

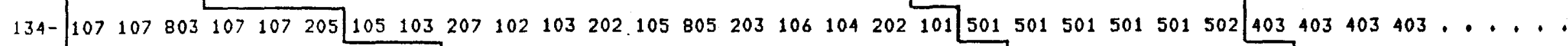

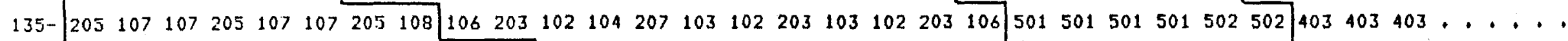

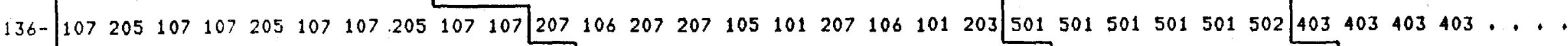

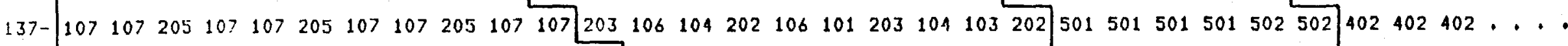

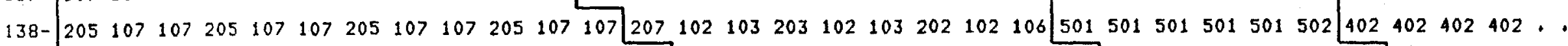

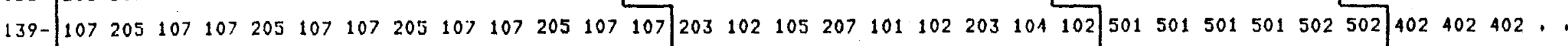

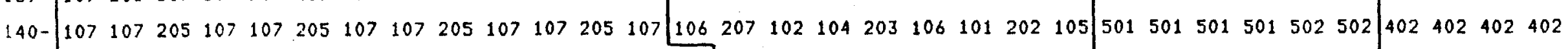

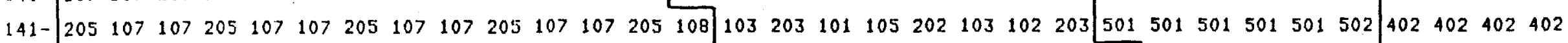

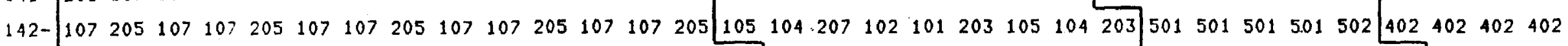

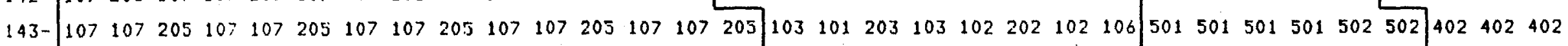

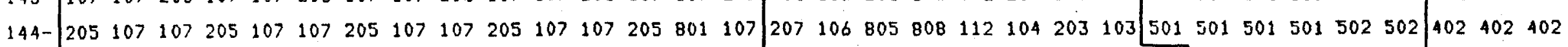

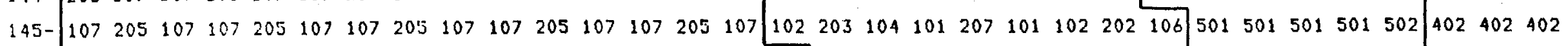

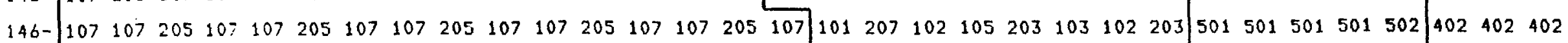

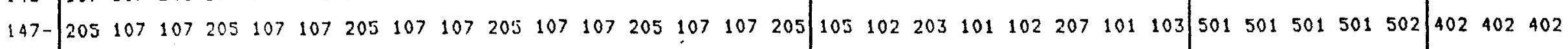

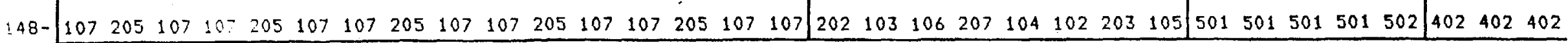




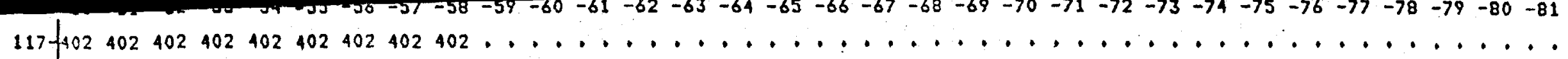

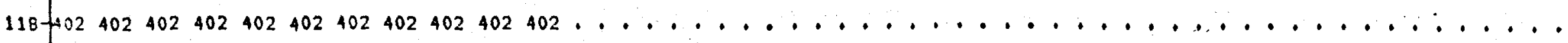

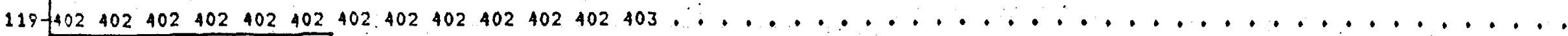
$120-502502502502502502402402402402402402403403403 \ldots \ldots \ldots \ldots$ $121-5015015015015025025025025025024402402403403403403404 \ldots \ldots \ldots$

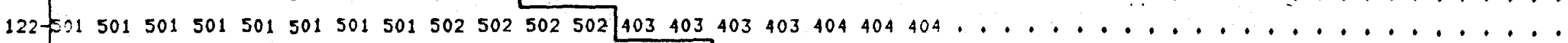

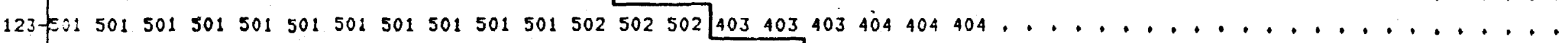

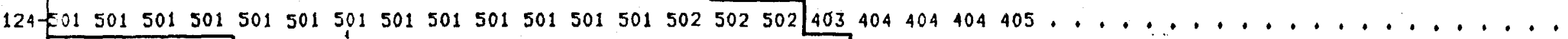

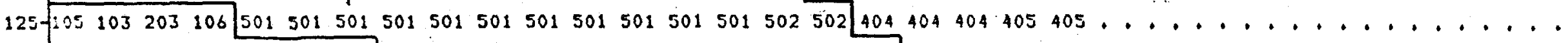
$126-203101102202103106203501501501501501501501501501502502$ 404 $404405405405 \ldots \ldots$. . . . . . . . . .

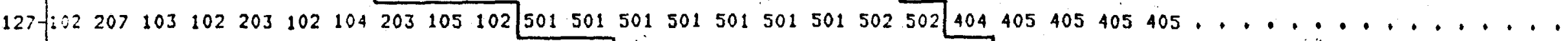

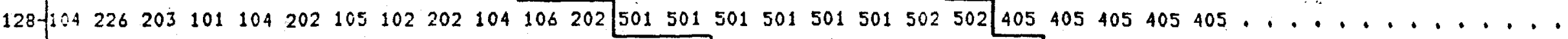
$129-2071011052071061022031032072031021032031065015015015015015025024404404404404404$. . . . . . . $130-1062031021012071032262021061022021041012031015015015015015015025024404404404404404$. . . . . . .

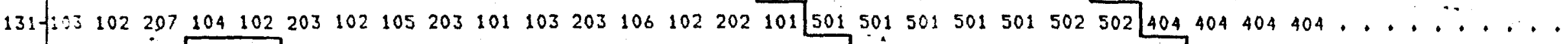

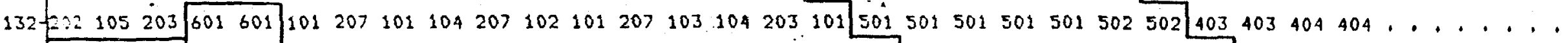

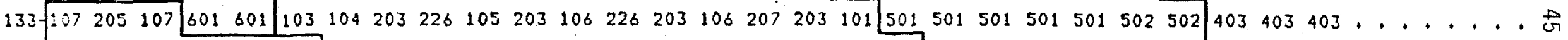

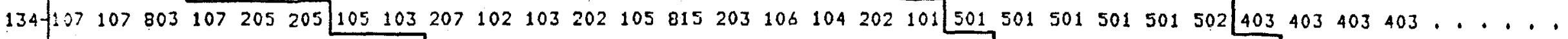

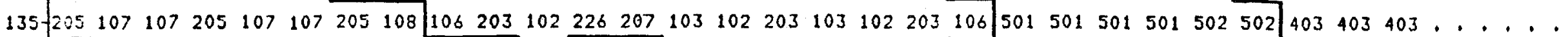

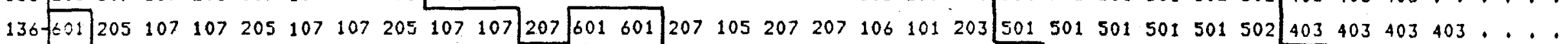

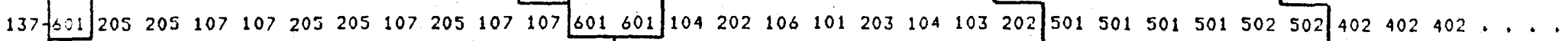

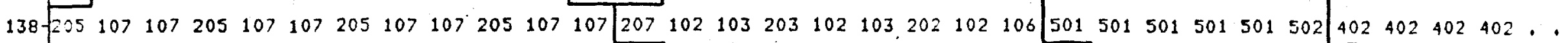

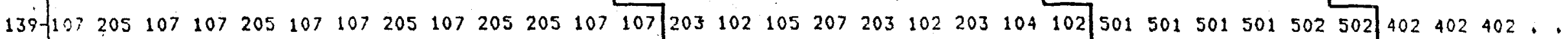

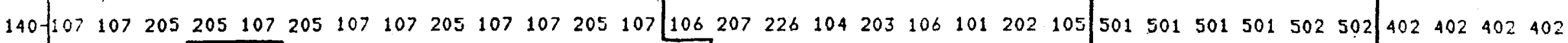

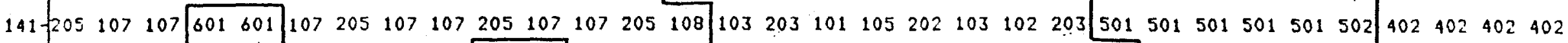

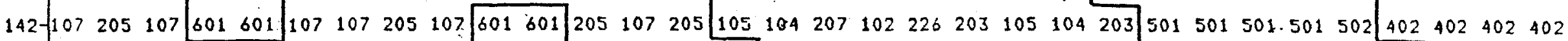

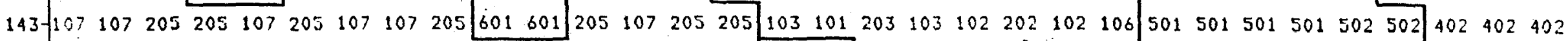

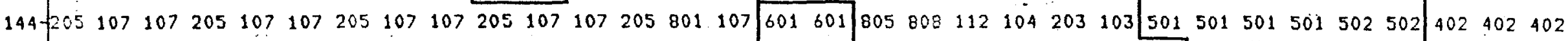

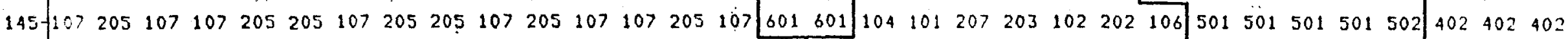

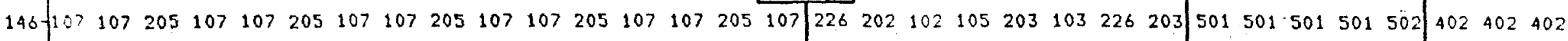

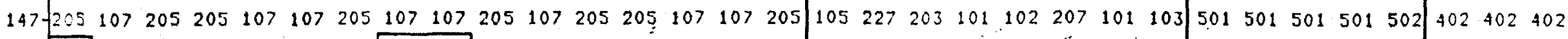

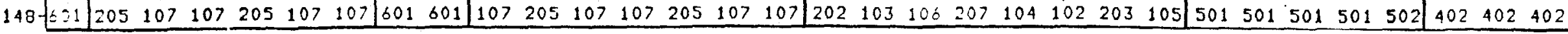

Fig. 4.3 The xyz Calculational Model for ZPPR-17B. 


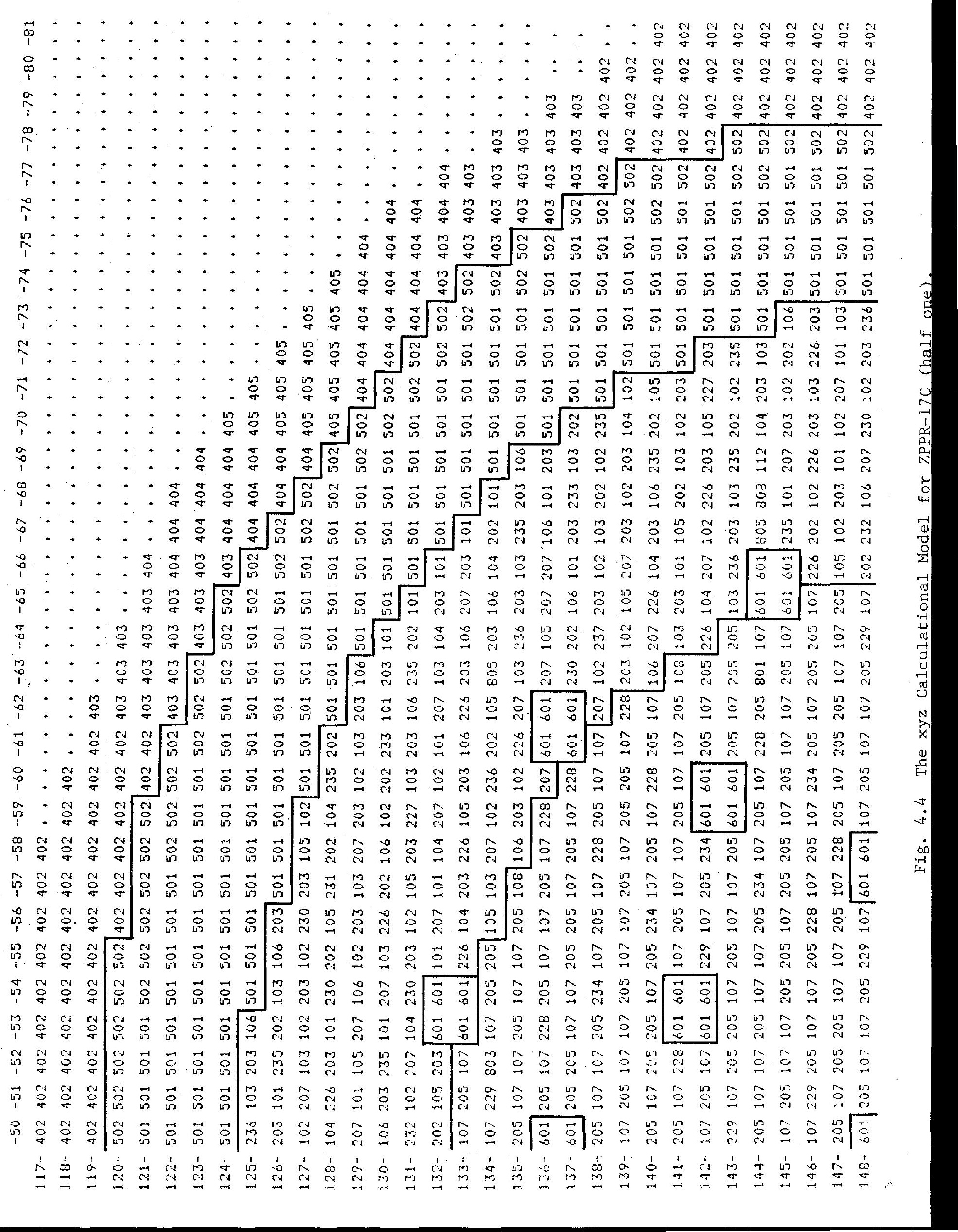




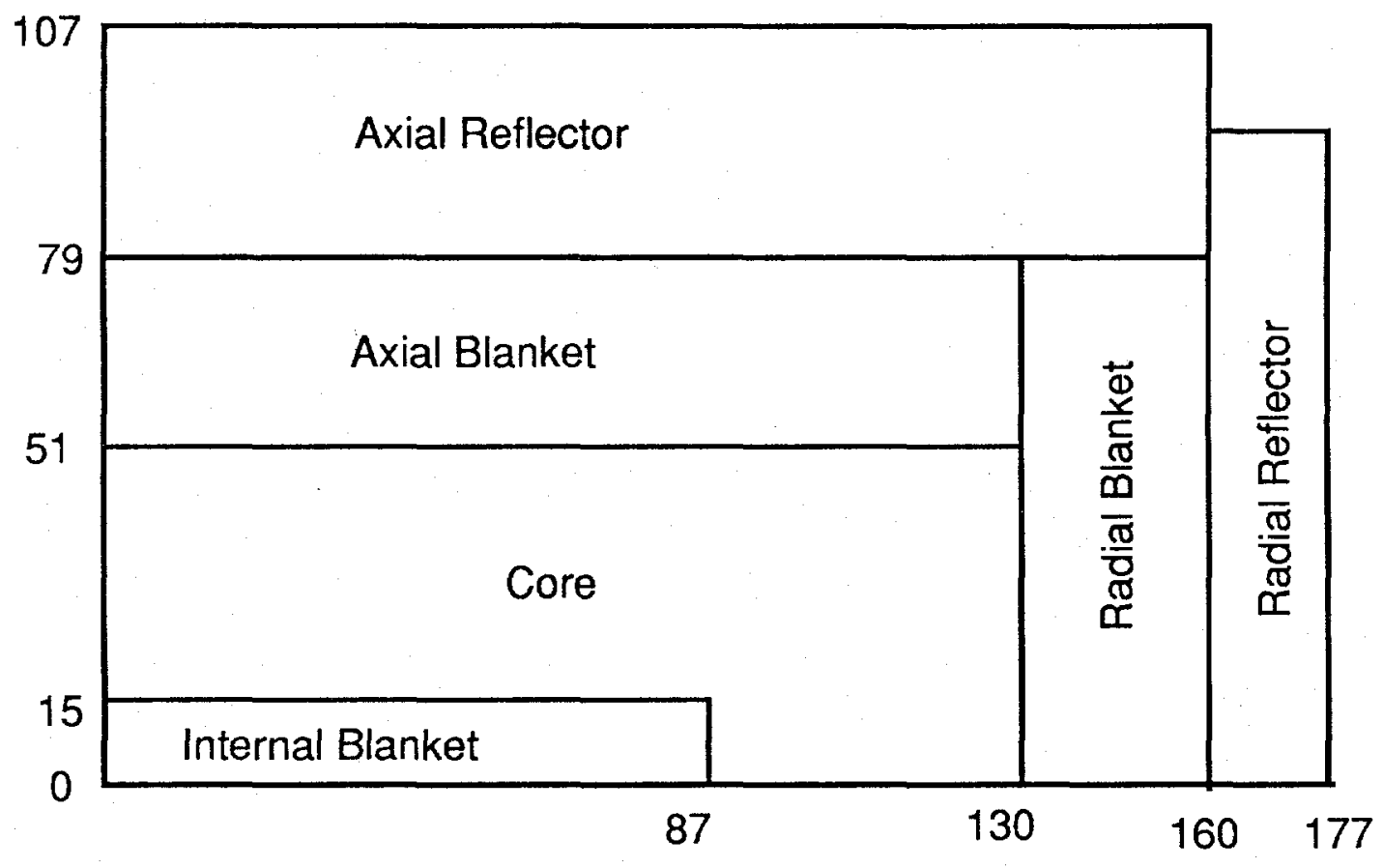

Dimensions in $\mathrm{cm}$

Fig. 4.5 rz Diagram of ZPPR-17^. 


\section{CALCULATED EIGENVALUES AND EFFECTIVE DELAYED NEUTRON FRACTIONS}

\subsection{Experimental Reactivities and Nodal Diffusion Results}

Both critical and subcritical references were established for each phase of $Z P P R-17$. The excess reactivities with operating shim rods withdrawn were measured for the critical reference by the positive period technique and for the subcritical reference by inverse kinetics analysis of a rod drop. Cross sections for ZPPR analysis are conventionally processed for a temperature of $293 \mathrm{~K}$. Experimental excess reactivities are adjusted to this temperature using measured temperature coefficients and converted to $\Delta k$ units using the calculated $\beta$-effective values. No adjustment is made for the absolute interface gap, but adjustment to a reference half-separation and ${ }^{241} \mathrm{Pu}$ decay date is made for comparison of reactivities. Tables 5.1 to 5.3 show the experimental k-effective values for the critical and subcritical references of $Z P P R-17 \mathrm{~A}, 17 \mathrm{~B}$, and $17 \mathrm{C}$ and a comparison with the nodal diffusion calculations using xyz geometry and 21 group ENDF/B-V.2 data.

The uncertainties shown in the tables result only from the statistics of the kinetics analysis and reactivity coefficients. The total uncertainty is much larger. For ZPPR-17, this will be close to that evaluated for ZPPR-13, since the principal source of uncertainty is the knowledge of the fuel masses. This total uncertainty on the experimental k-effective values is close to $0.00050(10)$.

For $2 P P R-17 \mathrm{~A}$, the difference between critical and subcritical reference reactivities is due to replacement of a double-fuel-column drawer in location 136-62 and the seven symmetric matrix positions (total $4.35 \mathrm{~kg}$ $\left.{ }^{239} \mathrm{Pu}\right)$. This reactivity difference is $0.001099 \Delta \mathrm{k} \pm 0.000039 \Delta \mathrm{k}$ assuming that all uncertainties, other than those in Table 5.1, are closely correlated. The calculated difference is $0.001187 \mathrm{giving}$ a $\mathrm{C} / \mathrm{E}$ value of 1.080. This result is consistent with that obtained for analysis of fuel worths.

The nodal diffusion k-effective for $17 \mathrm{~B}$ is $0.2 \%$ lower than that for $17 \mathrm{~A}$. This result is attributed to overestimation of leakage in CRPs by 
diffusion theory and is consistent with results for several other ZPPR cores.

\subsection{Results for Other Calculation Models}

Several calculations, in addition to the reference nodal diffusion model (NDT), were made for different analyses. A nodal transport (NTT) calculation with shim rods (WSH) inserted was made for reaction rate analysis. A finite-difference diffusion (FDDT) calculation was made for B-effective. Six group (6G) xyz calculations were made for control rod studies and $r z$ calculations were made for initial analysis of reactivitiy coefficients. The k-effective values from these models used a $55 \mathrm{~mm}$ mesh in xyz geometry and a similar mesh in $r z$ geometry. The nodal diffusion models used coarse $z$ nodes of 70 to $150 \mathrm{~mm}$ (and a $55 \mathrm{~mm}$ node spacing in the $\mathrm{xy}^{-}$ plane in order to represent the ZPPR geometry). These calculations have a very small truncation error. Results based on the different models are given in Tables 5.4 to 5.6. The effects of several modelling approximations for 17A are shown in Table 5.7. Modelling effects for ZPPR-17B are similar to those found for ZPPR-17A. The worth of the operating shim rods inserted to the core/blanket interface is $+0.0415 \% \Delta \mathrm{k}$. The error due to the use of coarse mesh (about $55 \mathrm{~mm}$ ) in finite difference diffusion calculations is $+0.206 \% \Delta \mathrm{k}$ in 21 groups and $+0.221 \% \Delta$ in 6 groups. The nodal transport correction to the nodal diffusion k-effective for ZPPR-17B is $+0.497 \%$. the increased transport correction compared with that for ZPPR-17A $(+0.406 \%)$ is attributed to the overestimate of leakage in the CRP banks by diffusion calculations.

The modelling effects derivable from the present calculations for ZPPR-17C are only approximate due to differences in core models. The transport correction to the nodal diffusion k-effective is $+0.57 \% \Delta \mathrm{k}$ taking into account a correction of $+0.04 \% \Delta k$ for difference in the shim rod positions (calculated for ZPPR-17B). This compares with a transport correction of $+0.50 \% \Delta \mathrm{k}$ calculated for ZPPR-17B. ${ }^{2}$ The error due to coarse mesh size in FDDT appears to be $+0.58 \% \Delta k$. However, differences of one or two tenths of a percent may arise from use of generic master compositions in 
FDDT models. Modelling effects that are somewhat different to those for ZPPR-17B are to be expected due to the presence of the control rods.

For ZPPR-17A, a noteworthy discrepancy exists between diffusion to transport corrections calculated by the nodal codes in xyz geometry and by the finite difference codes in $r z$ geometry. The nodal transport calculation predicts a transport effect of $+0.41 \%$ while the $\mathrm{rz}$ models predict $+0.60 \%$. A similar difference was found for ZPPR-17B from a comparison of an $\mathrm{S}_{4}$ calculation made in xyz geometry with the TRITAC code 12 with the nodal transport result.

\subsection{Summary of Eigenvalue Analysis}

Fuel and ${ }^{238} \mathrm{U}$ loadings for ZPPR-17 are shown in Table 5.8. The fuel enrichment increased from $14.9 \%$ in $17 \mathrm{~A}$ to $15.7 \%$ in $17 \mathrm{C}$ which was critical with 13 control rods half inserted. The calculated $k$ ef s were obtained from xyz geometry models with 21 group cross sections. Nodal diffusion results were relatively low, 0.987 for the simple core $17 \mathrm{~A}, 0.984$ in $17 \mathrm{~B}$ (due to an overestimate of leakage in the CRPs) and 0.985 in $17 \mathrm{C}$. Nodal transport results were $0.4 \%$ to $0.6 \%$ higher. The $\mathrm{S}_{4}$ transport calculations were made for $17 \mathrm{~A}$ using the TWODANT code in rz geometry and for 17B using the TRITAC code in xyz geometry.11,12 These showed significantly larger transport corrections; $0.6 \%$ in $17 \mathrm{~A}$ and $0.8 \%$ in $17 \mathrm{~B}$, than the nodal transport values. For $17 \mathrm{C}$, the $\mathrm{S}_{4}$ transport correction was estimated from six-group calculations for the subcritical core with control rods inserted.

The nodal transport results for ZPPR-17 appear to be somewhat less accurate than in other homogeneous and radially heterogeneous cores. Previous tests of the code for ZPPR-13 showed that over $80 \%$ of the diffusion theory error was eliminated. ${ }^{3}$ The $S_{4}$ results, with a bias of 0.993 , are consistent with other results for LMR cores. ${ }^{14}$

\subsection{Delayed Neutron Fractions}

Delayed neutron parameters were calculated with the 21 group $x y z$ models. The finite-difference solutions were used since at the start of the 
analysis the editing of the nodal solutions in the VARI3D code has not been fully tested. Results are given in Tables 5.9 to 5.11. The calculated $B_{\text {eff }}$ s decrease slightly as the enrichment increases from $17 \mathrm{~A}$ to $17 \mathrm{C}$. 
TABLE 5.1 Experimental and Calculated k-effective Results for ZPPR-17A

Critical Reference

Date

Reactor Run Number

Temperature, $\mathrm{K}$

Interface Gap, GIU ${ }^{a}$

Measured Excess Reactivity, $\phi$

Adjustment to $293 \mathrm{~K}, \phi^{\mathrm{b}}$

Adjustment to $44.2 \mathrm{GIU}^{\mathrm{C}}, \phi$

Adjustment to $02 / 23 / 87, \phi$

Adjusted Reactivity, $\phi$

Experimental K-effective (E) ${ }^{d}$

Calculation, 21G XYZ NDT (C)

$C / E$ for k-effective
$02 / 23 / 87$

22

300.3

$-44.2$

$+7.1 \pm 0.1$

$+7.88 \pm 0.80$

$-\cdots$

$+15.0 \pm 0.8$

$1.000508 \pm 0.000027$

0.987075

0.986574

Subcritical Reference

$02 / 26 / 87$

25

300.47

$-44.3$

$-25.86 \pm 0.21$

$+8.07 \pm 0.82$

$+0.34 \pm 0.04$

$+0.072 \pm 0.007$

$-17.38 \pm 0.85$

$0.999412 \pm 0.000029$

0.985888

0.986468

$\mathrm{a}_{\mathrm{GIU}}=\mathrm{Gap}$ interface unit (approximately 1 mil) on a scale with arbitrary zero.

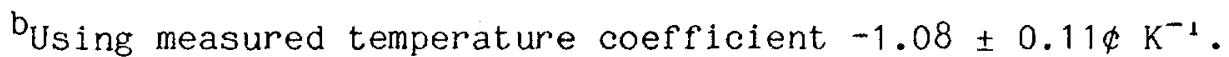

${ }^{c}$ Using measured gap coefficient $-0.18 \pm 0.02 \notin \mathrm{GIU}^{-1}$.

$d_{\text {Using calculated }}{ }^{241} \mathrm{Pu}$ decay coefficient $-0.02386 \notin$ day $^{-1}$.

$e_{U s i n g}$ calculated $\beta$-effective 0.003385 . 
TABLE 5.2 Experimental and Calculated k-effective Results for ZPPR-17B

\section{Critical Reference}

\section{Date}

Reactor Run Number

Temperature, $\mathrm{K}$

Interface Gap, mil ${ }^{\mathrm{a}}$

Measured Excess Reactivity, $\phi$

Adjustment to $293 \mathrm{~K}, \phi^{\mathrm{b}}$

Adjustment to $42.1 \mathrm{mil}{ }^{\mathrm{C}}, \phi$

Adjustment to $5 / 8 / 87, \phi^{d}$

Adjusted Reactivity, $\phi$

Experimental K-effective $(E)^{e}$

Calculation, $21 \mathrm{G} X Y Z$ NDT

$C / E$ for k-effective
$5 / 8 / 87$

96

300.1

42.1

$+11.7 \pm 0.1$

$+7.67 \pm 0.78$

- -

$+19.4 \pm 0.8 \phi$

$1.000655 \pm 0.000027$

0.984989

0.984344

Subcritical Reference

an a scale with arbitrary zero.

$\mathrm{b}_{\mathrm{Using}}$ measured temperature coefficient $-1.08 \pm 0.11 \not \mathrm{K}^{-1}$.

${ }^{C}$ Using measured gap coefficient $-0.18 \pm 0.02 \phi \mathrm{mil}^{-1}$.

$\mathrm{d}_{\text {Using calculated }}{ }^{2+1} \mathrm{Pu}$ decay coefficient $-0.02386 \phi \mathrm{day}^{-1}$.

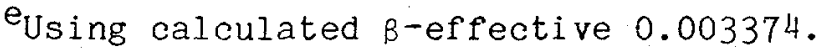


TABLE 5.3 Experimental and Calculated k-effective Results for ZPPR-17C

\section{Critical Reference}

Date

Reactor Run Number

Temperature, $\mathrm{K}$

Interface Gap, mil ${ }^{a}$

Measured Excess Reactivity, $\phi$

Adjustment to $293 \mathrm{~K}, \phi^{\mathrm{b}}$

Adjustment to $40.1 \mathrm{mil}^{\mathrm{c}}$, $\phi$

Adjustment to $6 / 16 / 87, \phi d$

Adjusted Reactivity, $\phi$

Experimental K-effective (E)

Calculation, $21 \mathrm{G} \mathrm{XYZ} \mathrm{NDT} \mathrm{(C)}$

C/E for k-effective
$6 / 16 / 87$

152

298.93

40.1

$+6.7 \pm 0.1$

$+6.40 \pm 0.6$

$-\cdots$

$+13.1 \pm 0.7$

$1.000440 \pm 0.000023$

0.984640

0.984207

Subcritical Reference

${ }^{a}$ on a scale with arbitrary zero.

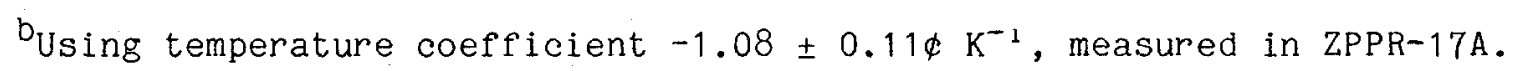

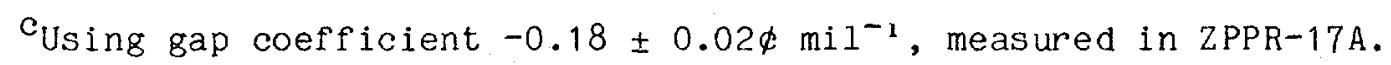

$\mathrm{d}_{\text {Using }}{ }^{241} \mathrm{Pu}$ decay coefficient $-0.02386 \notin \mathrm{day}^{-1}$, calculated for ZPPR-17A.

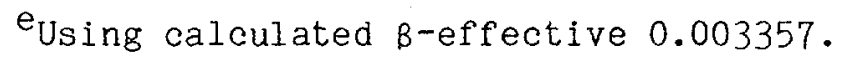


TABLE 5.4 k-effective Calculations for Several Models of ZPPR-17A

\begin{tabular}{|c|c|c|c|c|c|c|c|c|}
\hline & $\begin{array}{l}\text { Reactor } \\
\text { Loading } \\
\end{array}$ & $\begin{array}{c}\text { Number } \\
\text { of Groups } \\
\end{array}$ & Geometry & Mesh & $\begin{array}{l}\text { Calculation } \\
\text { Method }\end{array}$ & Streaming & Shim Rods ${ }^{\mathrm{C}}$ & $k$-effectived \\
\hline 1 & $\mathrm{C}$ & 21 & $x y z$ & $32 \times 32 \times 9$ & NDT & Yes & No & 0.987075 \\
\hline 2 & S & 21 & $x y z$ & $"$ & NDT & Yes & No & 0.985888 \\
\hline 3 & C & 21 & $x y z$ & $"$ & NDT & Yes & Yes & 0.986869 \\
\hline 4 & C & 21 & $x y z$ & $"$ & NTT & Yes & Yes & 0.990930 \\
\hline 5 & C & 21 & xyz & $32 \times 32 \times 20$ & FDDT & Yes & No & 0.989820 \\
\hline 6 & C & 6 & xyz & $32 \times 32 \times 9$ & NDT & Yes & No & 0.987662 \\
\hline 7 & $S$ & 6 & $x y z$ & " & NDT & Yes & No & 0.986489 \\
\hline 8 & C & 21 & $r z$ & $40 \times 20$ & EDDT & Yes & No & 0.988684 \\
\hline 9 & C & 21 & $\mathrm{rz}$ & $40 \times 20$ & FDDT & No & No & 0.992676 \\
\hline 10 & $\mathrm{C}$ & 21 & $r z$ & $64 \times 40$ & FDDT & No & No & 0.991398 \\
\hline 11 & C & 21 & $r z^{e}$ & $\infty$ & EDDT & No & No & 0.990946 \\
\hline 12 & $\mathrm{C}$ & 21 & $r z$ & $40 \times 20$ & S4 & No & No & 0.996979 \\
\hline 13 & C & 6 & $r z$ & $40 \times 20$ & FDDT & No & No & 0.992986 \\
\hline 14 & C & 21 & $x y z$ & $32 \times 32 \times 9$ & NDT & No & No & 0.990657 \\
\hline
\end{tabular}

$a_{C}=$ critical reference, $s=$ subcritical reference.

$b_{\text {NDT }}=$ nodal diffusion, NTT $=$ nodal transport, $F D D T=$ finite difference diffusion, $54=$ transport, S4 quadrature, TWODANT code.

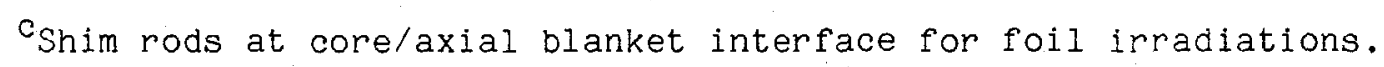

$d_{\text {Not }} c / E$ values.

Extrapolated to infinite mesh by $1 / n^{2}$ variation. 
TABLE 5.5 k-effective Calculations for Several Models of ZPPR-17B

\begin{tabular}{|c|c|c|c|c|c|c|c|c|}
\hline & $\begin{array}{l}\text { Reactor } \\
\text { Loading } \\
\end{array}$ & $\begin{array}{l}\text { Number } \\
\text { of Groups } \\
\end{array}$ & Geometry & Mesh & $\begin{array}{l}\text { Calculation } \\
\text { Method } \\
\end{array}$ & Streaming & Shim Rods ${ }^{c}$ & $\underline{k-e f f e c t i v e}$ \\
\hline 1 & C & 21 & $x y z$ & $32 \times 32 \times 9$ & NDT & Yes & No & 0.984989 \\
\hline 2 & $\mathrm{C}$ & 21 & $x y z$ & $"$ & NDT & Yes & Yes & 0.984574 \\
\hline 3 & $\mathrm{C}$ & 21 & $x y z$ & $"$ & NTT & Yes & Yes & 0.989548 \\
\hline 4 & C & 21 & $x y z$ & $32 \times 32 \times 20$ & FDDT & Yes & No & 0.987051 \\
\hline 5 & C & 6 & $x y z$ & $"$ & FDDT & Yes & No & 0.986379 \\
\hline 6 & $S$ & 6 & $\mathrm{xyz}$ & $32 \times 32 \times 9$ & NDT & Yes & No & 0.984174 \\
\hline 7 & $S$ & 6 & $x y z$ & $32 \times 32 \times 20$ & FDDT & Yes & No & 0.985234 \\
\hline 8 & C & 21 & $r z$ & $50 \times 20$ & FDDT & Yes & No & 0.984383 \\
\hline 9 & C & 6 & $r z$ & $50 \times 20$ & FDDT & Yes & No & 0.984196 \\
\hline
\end{tabular}

${ }^{a} \mathrm{C}=$ critical reference, $S=$ subcritical reference.

$\mathrm{b}_{\mathrm{NDT}}=$ nodal diffusion, NTT = nodal transport, $\mathrm{FDDT}=$ finite difference diffusion, $S_{4}=$ transport, $S_{4}$ quadrature, TWODANT code.

CShim rods at core/axial blanket interface for foil irradiations.

$\mathrm{d}_{\text {Not }} \mathrm{C} / \mathrm{E}$ values. 
TABLE 5.6 k-effective Calculations for Several Models of ZPPR-17C

\begin{tabular}{|c|c|c|c|c|c|c|c|c|}
\hline & $\begin{array}{l}\text { Reactor } \\
\text { Loading } \\
\end{array}$ & $\begin{array}{l}\text { Number } \\
\text { of Groups }\end{array}$ & Geometry & Mesh & $\begin{array}{l}\text { Calculation } \\
\text { Method } \\
\end{array}$ & Streaming & Shim Rods & k-effective \\
\hline 1 & $c$ & 21 & $x y z$ & $32 \times 32 \times 18$ & NDT & Yes & No & 0.984640 \\
\hline 2 & $\mathrm{C}$ & 21 & $x y z$ & $32 \times 32 \times 18$ & NTT & Yes & Yes & 0.989924 \\
\hline 3 & C. & 21 & $x y z$ & $32 \times 32 \times 40$ & FDDT & Yes. & No & 0.990465 \\
\hline 4 & C. & 6 & $x y z$ & $32 \times 32 \times 40$ & FDDT & Yes & No & 0.988263 \\
\hline 5 & $c$ & 21 & $r z$ & $50 \times 40$ & FDDT & Yes & No & 0.980588 \\
\hline
\end{tabular}

${ }^{a} \mathrm{C}=$ critical reference.

$b_{N D T}=$ nodal diffusion, NTT = nodal transport, $F D D T=$ finite difference diffusion.

${ }^{\circ}$ Shim rods at core/axial blanket interface for foil irradiations.

$\mathrm{d}_{\text {Not }} \mathrm{C} / \mathrm{E}$ values. 
TABLE 5.7 Modelling Effects Calculated for ZPPR-17A

\begin{tabular}{|c|c|c|c|}
\hline Correction & & $\begin{array}{c}\text { Table } 5.4 \\
\text { Cases } \\
\end{array}$ & $\begin{array}{c}\Delta \mathrm{k} \\
\text { Value }\end{array}$ \\
\hline $\begin{array}{l}\mathrm{rz} \rightarrow \mathrm{xy} \text { Geometry } \\
6 \text { Groups } \rightarrow 21 \text { Groups }\end{array}$ & $\begin{array}{l}x y z \\
x y z \\
r z\end{array}$ & $\begin{aligned} 8 & \rightarrow 5 \\
6 & \rightarrow 1 \\
7 & \rightarrow 2 \\
13 & \rightarrow 9\end{aligned}$ & $\begin{array}{l}+0.001136^{a} \\
-0.000587 \\
-0.000601 \\
-0.000310^{a}\end{array}$ \\
\hline Mesh in FDDT & $\begin{array}{l}x y z \\
\mathrm{rz}\end{array}$ & $\begin{array}{l}5 \rightarrow 1 \\
9+11\end{array}$ & $\begin{array}{l}-0.002745^{b} \\
-0.001730\end{array}$ \\
\hline Streaming & $\begin{array}{l}x y z \\
r z\end{array}$ & $\begin{array}{c}14 \rightarrow 1 \\
9 \rightarrow 8\end{array}$ & $\begin{array}{l}-0.003582 \\
-0.003992^{a}\end{array}$ \\
\hline Shim Rod Worth & $x y z$ & $1 \rightarrow 3$ & $-0.000206^{\mathrm{C}}$ \\
\hline Diffusion $\rightarrow$ Transport & $\begin{array}{l}x y z \\
r z\end{array}$ & $\begin{aligned} 3 & \rightarrow 4 \\
11 & \rightarrow 12\end{aligned}$ & $\begin{array}{l}+0.004061 \\
+0.006033\end{array}$ \\
\hline
\end{tabular}

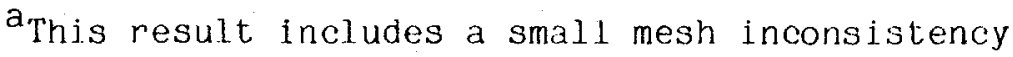
between 40 radial points and 32 xy points.

${ }^{b}$ Assuming nodal diffusion solution has a negligible mesh error.

${ }^{c}$ Calculated reactivity for 8 shim rods inserted to core/axial blanket interface relative to the fully withdrawn position, model used for calculations of reaction rate distributions. 
TABLE 5.8 ZPPR-17 Fuel Loadings and Criticality Predictions

\begin{tabular}{|c|c|c|c|}
\hline & $17 \mathrm{~A}$ & $17 \mathrm{~B}$ & $17 \mathrm{C}$ \\
\hline $\begin{array}{l}\text { Fissile } \mathrm{Pu}, \mathrm{kg} \\
2{ }^{38} \mathrm{U}, \mathrm{kg}\end{array}$ & 2272 & 2247 & 2450 \\
\hline Core & 12659 & 12248 & 12815 \\
\hline Internal Blanket & 2375 & 2218 & 2218 \\
\hline Fuel Enrichment, $\%$ & 14.9 & 15.2 & 15.7 \\
\hline \multicolumn{4}{|l|}{ Calculated $\mathrm{k}_{\text {eff }}$} \\
\hline Nodal Diffusion & 0.9866 & 0.9843 & 0.9846 \\
\hline Nodal Transport & 0.9906 & 0.9893 & 0.9903 \\
\hline $\mathrm{S}_{4}$ Transport & 0.9926 & 0.9924 & 0.9934 \\
\hline
\end{tabular}


TABLE 5.9 Delayed Neutron

Parameters for ZPPR-17A

\begin{tabular}{lllll}
\hline \multirow{2}{*}{ Family } & \multicolumn{1}{c}{$a_{i}$} & $\lambda_{i}, s^{-1}$ & $\beta_{i}, \%$ \\
\cline { 5 - 5 } 1 & & 0.02420 & 0.01298 & 0.00819 \\
2 & 0.20807 & 0.03144 & 0.07043 \\
3 & 0.18628 & 0.13560 & 0.06306 \\
4 & 0.36059 & 0.34601 & 0.12206 \\
5 & 0.16632 & 1.3679 & 0.05630 \\
6 & 0.05455 & 3.7597 & 0.01846 \\
\hline
\end{tabular}

B-effective $0.3385 \%$

Prompt neutron lifetime $4.660 \times 10^{-7} \mathrm{~s}$
TABLE 5.10 Delayed Neutron Parameters for ZPPR-17B

\begin{tabular}{|c|c|c|c|}
\hline Family & $a_{i}$ & $\lambda_{i}, s^{-1}$ & $\beta_{j}, \%$ \\
\hline 1 & 0.02433 & 0.01298 & 0.00821 \\
\hline 2 & 0.20868 & 0.03144 & 0.07040 \\
\hline 3 & 0.18655 & 0.13557 & 0.06293 \\
\hline 4 & 0.36032 & 0.34589 & 0.12216 \\
\hline 5 & 0.16576 & 1.3673 & 0.05592 \\
\hline 6 & 0.05436 & 3.7561 & 0.01834 \\
\hline
\end{tabular}

B-effective $0.3374 \%$

Prompt neutron lifetime $4.895 \times 10^{-7} \mathrm{~S}$

TABLE 5.11 Delayed Neutron Parameters for ZPPR-17C

\begin{tabular}{|c|c|c|c|}
\hline Family & $a_{i}$ & $\lambda_{i}, s^{-1}$ & $\beta_{i}, \%$ \\
\hline 1 & 0.02428 & 0.01298 & 0.00815 \\
\hline 2 & 0.20858 & 0.03144 & 0.07003 \\
\hline 3 & 0.18651 & 0.13562 & 0.06262 \\
\hline 4 & 0.36028 & 0.34589 & 0.12096 \\
\hline 5 & 0.16592 & 1.3673 & 0.05570 \\
\hline 6 & 0.05443 & 3.7570 & 0.01828 \\
\hline
\end{tabular}

$\beta$-effective $0.3357 \%$

Prompt neutron lifetime $4.235 \times 10^{-7} \mathrm{~S}$ 


\section{ANALYSIS OF REACTION RATE MEASUREMENTS}

Detailed measurements of fission rates in ${ }^{239} \mathrm{Pu},{ }^{2{ }^{35}} \mathrm{U}$ and ${ }^{2{ }^{3} 8} \mathrm{U}$ and capture in ${ }^{238} \mathrm{U}$ were made in the three critical $\mathrm{ZPPR}-17$ reference configurations. The standard ZPPR foil techniques were used. ${ }^{15}$ However, the gamma counting system was recalibrated using the latest reference data. ${ }^{16}$ This section presents the cell-average experimental results and comparisons with analysis. More detailed results were reported in references 3 and 5 .

The number of experimental reaction rate values is quite overwhelming: 623 cell-average values in $17 \mathrm{~A}, 536$ in $17 \mathrm{~B}$ and 1078 in $17 \mathrm{C}$. (Several hundred foil measurements were made in addition to obtain cellaverage/foil factors in different cells.). The results in this section are selected to give a summary of the analysis. Full details of the data are available in the references.

\subsection{Description of the Measurements}

Foil locations were chosen to emphasize the axial characteristics of the ZPPR-17 assemblies. Comparisons with calculations are provided for the cases of no CRPS or CRS (17A), of $25 \mathrm{CRPS}$ (17B) and of 13 half-inserted CRs $(17 \mathrm{C})$. The measurements fell into three basic categories:

(i) Radial distributions along the $x$-axis at $z=5 \mathrm{~cm}$ (through the internal blanket), at $z=18 \mathrm{~cm}$ (just above the internal blanket), and at $z=28 \mathrm{~cm}$ from the midplane. In $17 \mathrm{C}$, measurements were made at these axial displacements in both halves.

(ii) Radial distributions at $45^{\circ}$ to the axis, using ${ }^{235} \mathrm{U}$ foils, for the same three axial elevations. In $17 \mathrm{~A}$, these measurements were made at $z=5 \mathrm{~cm}$ only. In $17 \mathrm{C}$, measurements were made at the three axial displacements in both halves.

(iii) Axial distributions. In $\mathrm{ZPPR}-17 \mathrm{~A}$, these were in 148-50 (core center), 148-66 (peak flux region just outside the radial edge 
of the internal blanket) and in 148-70 (half way between internal and radial blankets). In ZPPR-17B, these were in 148-51 (adjacent to the central CRP), in 148-62 (adjacent to an outer-ring CRP) and in 148-70. In ZPPR-17C, the 17B measurements were repeated, but included both half-one and half-two.

The foil placement in ZPPR-17A in the $x y$ plane is shown in Fig. 6.1 and in an $\mathrm{rz}$ sense in Fig. 6.2. Placements for $17 \mathrm{~B}$ and $17 \mathrm{C}$ are shown in Fig. 6.3 .

The statistical uncertainties on the measured reaction rates are in the range $0.5 \%$ to $0.7 \%$ for fission in ${ }^{235} \mathrm{U}$ and ${ }^{239} \mathrm{Pu}$ and for capture in ${ }^{238} \mathrm{U}$, within the core and internal blanket regions, increasing to $0.9 \%$ in the axial blanket. For fission in ${ }^{2{ }^{38}} \mathrm{U}$, statistical uncertainties are about $1.5 \%$ in the fuel regions, $4 \%$ in the internal blanket and $4 \%$ (near core boundary) to $15 \%$ in the axial blanket. 


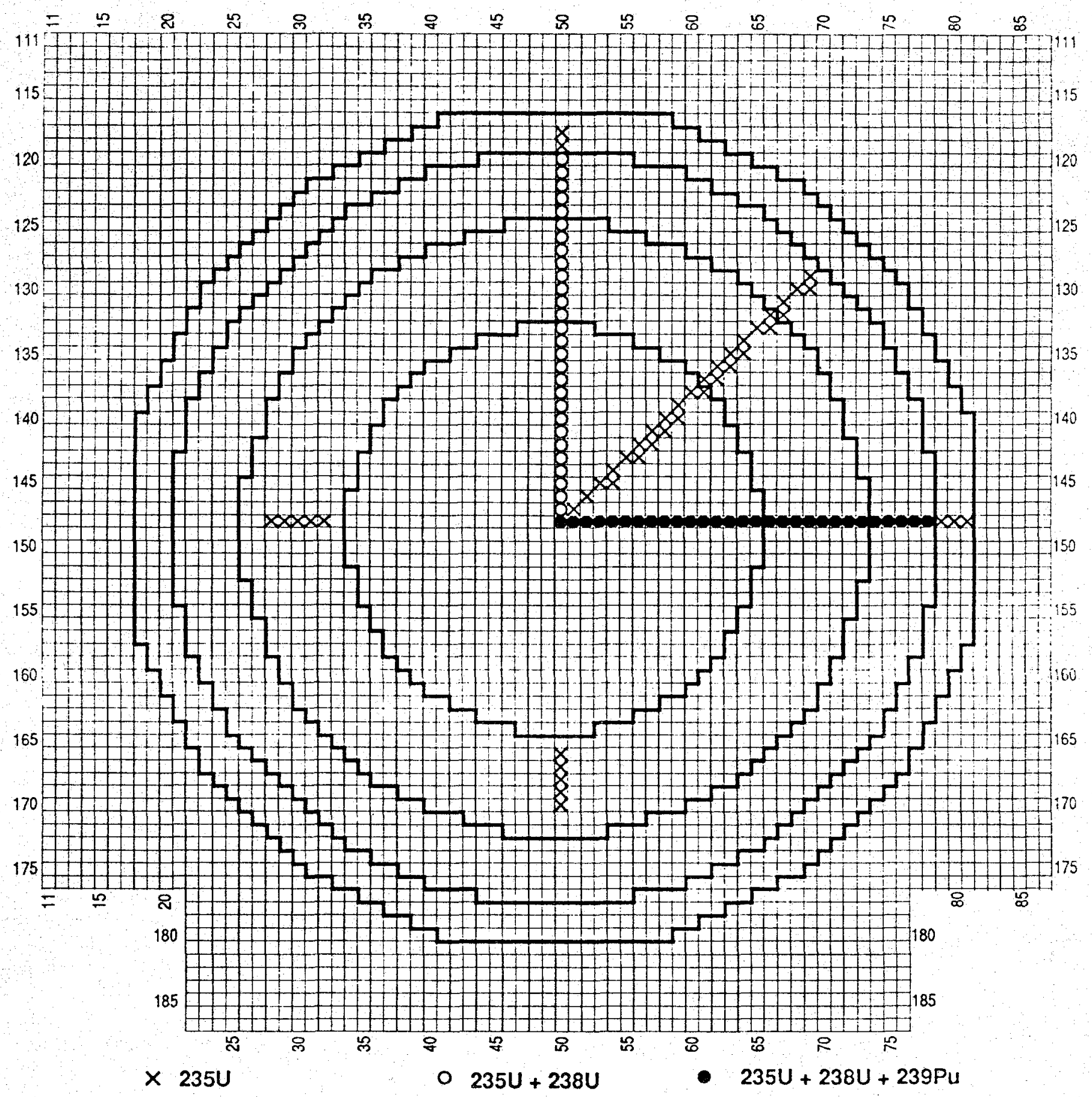

ZPPR-17A

Fig. 6.1. Foil Locations in ZPPR-17A in the $x y$ Plane. 


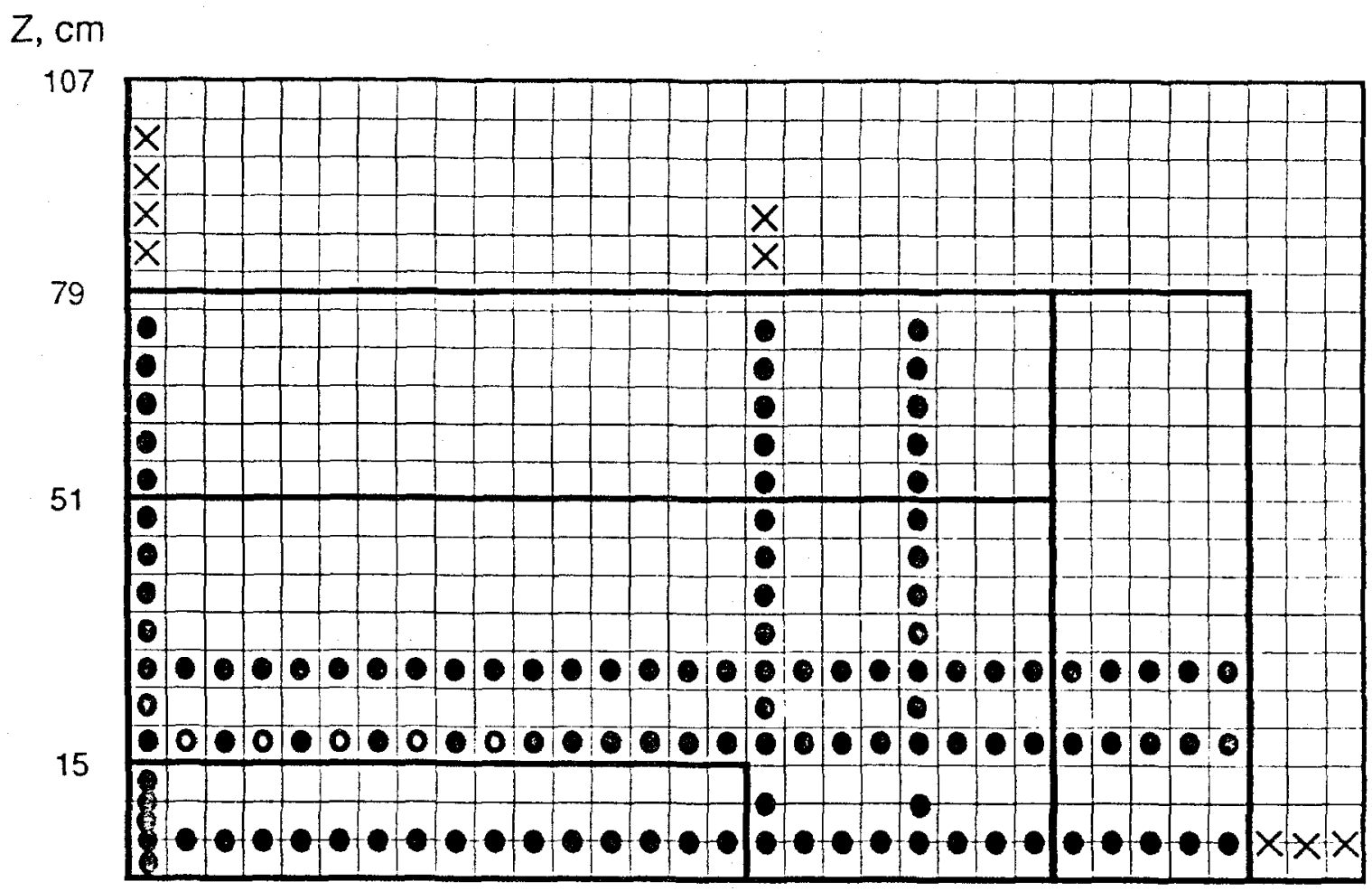

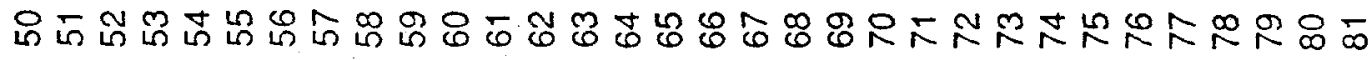
MATRIX POSITION

$235 U+238 U+239 P u$

- $\quad 235 U+238 U$

$x$

$235 \mathrm{U}$

Fig. 6.2. Foil Locations in ZPPR-17A in the $x z$ Plane. 


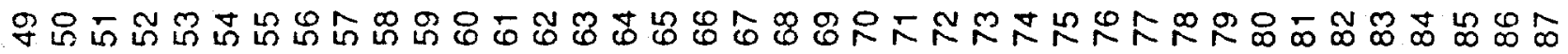

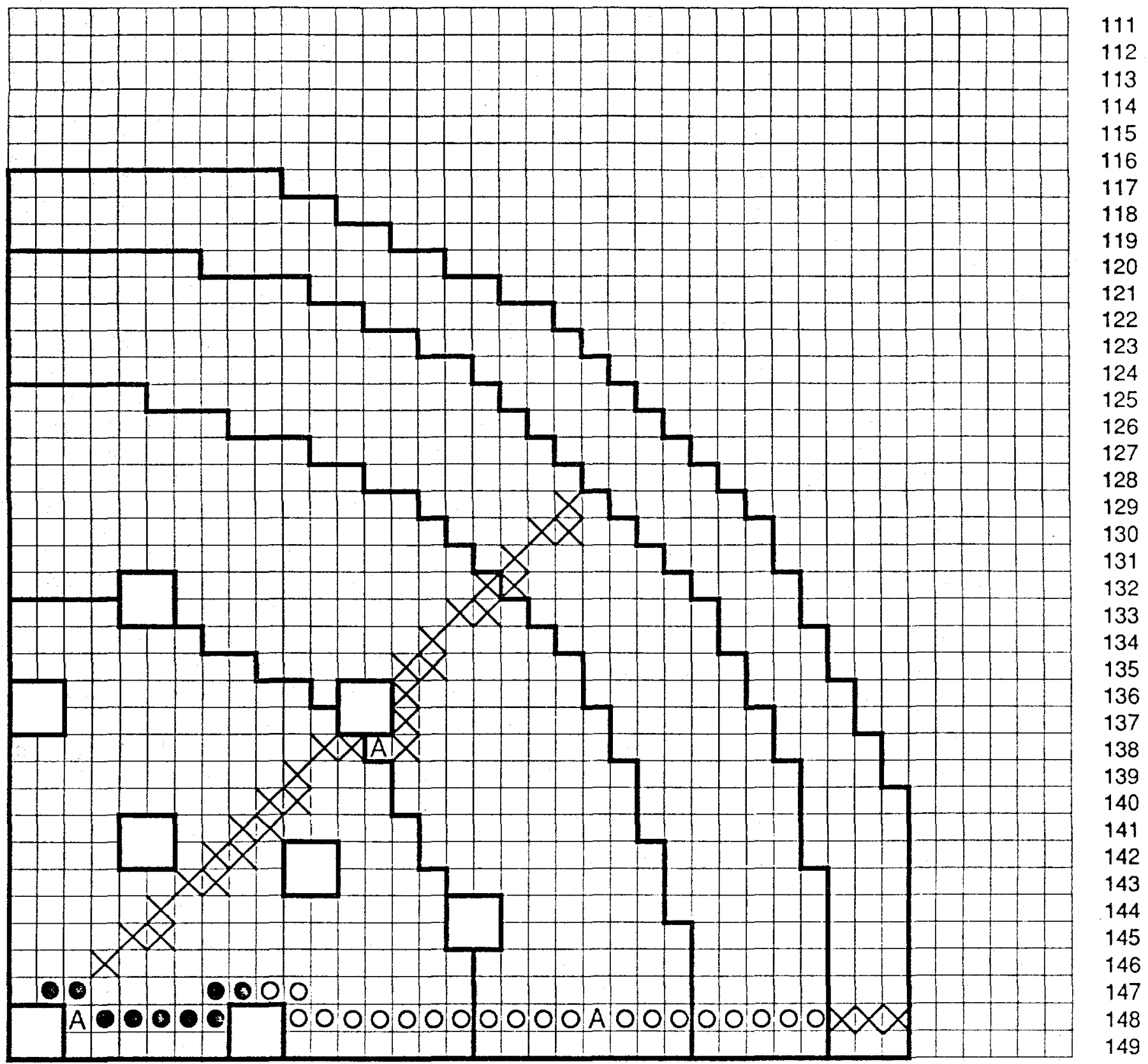

$X 235 U \quad \circ \quad 235 U+238 U \quad \bullet \quad 235 U+238 U+239 P U$

A Axial Traverse

Fig. 6.3 Foil Locations in $\mathrm{ZPPR}-17 \mathrm{~B}$ and $17 \mathrm{C}$ in the $\mathrm{xy}$ Plane 


\subsection{Calculations}

Calculations were made with ENDF/B-V.2 cross sections in 21 groups using an xyz model and the nodal transport path of the DIF3D code. The microscopic data were processed for cell heterogeneity using a bucklingrecycle technique (section 4.3). These cross sections were prepared for ZPPR-17A and used in $17 \mathrm{~B}$ and $17 \mathrm{C}$ without reprocessing. The nodal transport calculations for ZPPR-17A and 17B used a quartic polynomial approximation for the fluxes. However, since ZPPR-17C required a full-z model, a cubio polynomial approximation was used because of computer storage limitations.

The models for reaction rate analysis included the ZPPR operating shim rods at or close to their average insertion during the irradiations. The measurements used eight symmetrically inserted rods specifically for convenience in the analysis. Cross sections for the shim rods were selfshielded for the boron blade and averaged over a matrix area with a special model using the SDX code. Measured shim rod reactivities were $6 \phi$ in $17 \mathrm{~A}$, $11 \phi$ in $17 \mathrm{~B}$ and $2 \phi$ in $17 \mathrm{C}$. These reactivities were reproduced by calculation within $2 \phi$ which is adequate in view of the small overall perturbations to core fission rates of less than $1 \%$ except in the immediate vicinity of the control drawers.

Calculated reaction rates were interpolated to the foil locations using the nodal polynomial coefficients for each mesh region and energy group. It is recognized that this procedure is somewhat flawed since the coefficients are calculated to give the average flux in a node and internal values are only approximate. An alternative method using quadratic interpolation on the node-average fluxes gave less satisfactory results in ZPPR-17 due to the coarse axial node spacings in the $z$-dimension which were typically $10 \mathrm{~cm}$ to $15 \mathrm{~cm}$.

The appropriate cell-average cross sections were used for calculations of reaction rates. For plutonium fission in blanket regions, cross sections self-shielded for the foil thicknesses were calculated in a special SDX model. Previous tests showed that foil shielding effects for ${ }^{235} \mathrm{U}$ were small in core regions. This may not be the case for ${ }^{235} \mathrm{U}$ fission 
in the softer spectra of the depleted uranium blanket regions. The present analysis uses cell average cross sections for ${ }^{235} \mathrm{U}$ which are close to infinite dilution.

For comparison with experiment, the calculated data ( $C$ ) have been normalized to experiment $(E)$ to give an average $C / E$ of unity for all results available for plutonium fission in the core regions. In ZPPR-17A, 74 results for plutonium fission were available and the distributions covered most of the core region (Fig. 6.1). However, in $17 \mathrm{~B}$ and $17 \mathrm{C}$, fewer plutonium fission measurements were possible because of foil inventory limitations. In the latter two cores, plutonium fission measurements were made axially adjacent to the central CRP or CRP/CR and in a few radial positions near the core center. Consequently, the normalizations differ among the three cores because of the spatial mispredictions of reaction rates. The $\mathrm{C} / \mathrm{E}$ results for $17 \mathrm{~B}$ and $17 \mathrm{C}$ are about $3 \%$ higher than those in $17 \mathrm{~A}$.

The experimental results are normalized to a reactor power of approximately 1 watt using countrates on two boron-trifluoride chambers (EXP1 and EXP2 in the ZPPR jargon) positioned outside the core. Although the relative normalization between the results in the three cores is only approximate the experimental reaction shapes can be compared. AIl experimental reaction rates in the following tables are given in units of $10^{-18}$ reactions per atom per second at a power of 1 watt. 


\subsection{Reaction Rate Distributions in ZPPR-17A}

Experimental reaction rates and C/E results for distributions along the $x$-axis at about $5 \mathrm{~cm}$ from the axial midplane are shown in Table 6.1. Similar tables for distributions at $18 \mathrm{~cm}$ and $28 \mathrm{~cm}$ from the midplane plus results for ${ }^{235} \mathrm{U}$ fission along the $\mathrm{y}$-axis and at $45^{\circ}$ to the axes are given in reference 3. The results for fission in plutonium at $z=5$ and $z=28 \mathrm{~cm}$ are plotted in Fig. 6.4. Results for fission in ${ }^{238} \mathrm{U}$ at $z=5$ and $28 \mathrm{~cm}$ are plotted in Fig. 6.5 .

The results in Table 6.1 are generally typical of those throughout ZPPR-17 analysis:

(i) The C/E results increase with distance from the core center for all reactions.

(ii) The C/E resultis for ${ }^{238} \mathrm{U}$ capture and fission vary systematically by $2 \%$ to $3 \%$ between single- and double-fuel-column drawers.

(iii) ${ }^{238} \mathrm{U}$ fission rates are inconsistently predicted by $5 \%$ to $10 \%$ across the boundaries of fuel and blanket zones.

(iv) ${ }^{235} \mathrm{U}$ fission and ${ }^{238} \mathrm{U}$ capture are predicted consistently through the radial blanket up to the last drawer, while $\mathrm{C} / \mathrm{E}$ results for ${ }^{239} \mathrm{Pu}$ fission decrease by several percent with penetration. The $C / E$ results for ${ }^{238} \mathrm{U}$ fission decrease with distance into the radial blanket by $25 \%$ but the statistical uncertainties are comparable at these penetrations.

(v) ${ }^{235} \mathrm{U}$ fission is markedly overpredicted at the last position in the blanket and the first position in the steel reflector. Note that the results in the outer part of the reflector are meaningless because of the boundary condition in the calculations.

The radial reaction rate analysis is summarized in Table 6.2. The C/E results have been averaged for groups of four successive matrix 
positions along each traverse. This has the advantage of reducing the statistical fluctuations and shows clearly the monotonic increase in $\mathrm{C} / \mathrm{E}$ with distance from the center for all traverses and all reactions.

Since the axial reaction rate distributions are of major interest, these results are given in detail in Tables 6.3 for the core center, in Table 6.4 for matrix $148-66$ in the outer core adjacent to the internal blanket and in Table 6.5 for the center of the outer core. Plots of the axial distributions of ${ }^{239} \mathrm{Pu}$ and ${ }^{238} \mathrm{U}$ fission at the three locations are given in Figs. 6.6 and 6.7 .

Axial reaction rate distributions are well predicted through the internal blanket and core regions. Within a given zone, fluctuations of individual $\mathrm{C} / \mathrm{Es}$ from the mean are not significant in comparison with experimental statistics. Calculations of ${ }^{23} \mathrm{U}$ fission through the internal blanket and the core (Table 6.3 and Fig. 6.7) are remarkably good in view of the variation in fission rates of a factor of four.

Results in the axial blanket are similar to those from the radial blanket but more definitive due to the increased thickness. The C/Es for ${ }^{235} \mathrm{U}$ fission and ${ }^{238} \mathrm{U}$ capture are consistent up to the last position $(74 \mathrm{~cm}$ from the core midplane) while C/Es for ${ }^{239} \mathrm{Pu}$ fission and ${ }^{238} \mathrm{U}$ fission decrease with distance into the blanket. Predictions of ${ }^{235} \mathrm{U}$ fission in the steel axial reflector are high by about 10\% (Table 6.3). 
TABLE 6.1 ZPPR-17A: Radial Reaction Rate Distributions along the $X$-axis at $z=5$ cm

\begin{tabular}{|c|c|c|c|c|c|c|c|c|c|}
\hline \multirow[b]{2}{*}{ Matrix } & \multirow[b]{2}{*}{ Zone } & \multicolumn{2}{|c|}{${ }^{239} \mathrm{Pu}(\mathrm{n}, \mathrm{f})$} & \multicolumn{2}{|c|}{${ }^{235} U(n, f)$} & \multicolumn{2}{|c|}{$238 U(n, \gamma)$} & \multicolumn{2}{|c|}{${ }^{238} U(n, f)$} \\
\hline & & Exp. & $C / E$ & Exp. & $C / E$ & Exp. & $\mathrm{C} / \mathrm{E}$ & Exp. & $C / E$ \\
\hline $148 \quad 50$ & IB & 6.983 & 0.962 & 8.016 & 0.993 & 0.9636 & 1.019 & 0.0538 & 0.899 \\
\hline 14851 & IB & 7.045 & 0.959 & 8.109 & 0.997 & 0.9790 & 1.011 & 0.0428 & 1.003 \\
\hline $148 \quad 52$ & IB & 7.079 & 0.960 & 8.156 & 0.997 & 0.9766 & 1.020 & 0.0457 & 0.935 \\
\hline 14853 & IB & 7.090 & 0.967 & 8.168 & 1.004 & 0.9844 & 1.021 & 0.0439 & 0.982 \\
\hline 14854 & IB & 7.116 & 0.975 & 8.256 & 1.005 & 1.0020 & 1.015 & 0.0460 & 0.952 \\
\hline $148 \quad 55$ & IB & 7.211 & 0.976 & 8.329 & 1.011 & 1.0090 & 1.023 & 0.0456 & 0.967 \\
\hline 14856 & IB & 7.408 & 0.967 & 8.495 & 1.009 & 1.0230 & 1.028 & 0.0468 & 0.962 \\
\hline 14857 & IB & 7.492 & 0.976 & 8.650 & 1.011 & 1.0480 & 1.025 & 0.0437 & 1.058 \\
\hline 14858 & IB & 7.633 & 0.980 & 8.865 & 1.009 & 1.0700 & 1.028 & 0.0456 & 1.037 \\
\hline $148 \quad 59$ & IB & 7.813 & 0.982 & 9.022 & 1.015 & 1.0940 & 1.032 & 0.0472 & 1.041 \\
\hline 14860 & IB & 7.991 & 0.987 & 9.220 & 1.018 & 1.1320 & 1.026 & 0.0518 & 1.005 \\
\hline 14861 & IB & 8.115 & 0.998 & 9.379 & 1.024 & 1.1460 & 1.042 & 0.0580 & 0.955 \\
\hline $148 \quad 62$ & IB & 8.295 & 1.002 & 9.517 & 1.028 & 1.1750 & 1.042 & 0.0614 & 0.998 \\
\hline $148 \quad 63$ & IB & 8.467 & 1.003 & 9.582 & 1.033 & 1.1890 & 1.048 & 0.0704 & 1.007 \\
\hline $148 \quad 64$ & IB & 8.522 & 1.009 & 9.509 & 1.037 & 1.2030 & 1.043 & 0.0799 & 1.081 \\
\hline $148 \quad 65$ & IB & 8.368 & 1.024 & 9.241 & 1.037 & 1.1910 & 1.036 & 0.1119 & 1.059 \\
\hline 14866 & $O C D$ & 7.925 & 1.013 & 8.680 & 1.022 & 1.1250 & 1.068 & 0.1997 & 1.004 \\
\hline $148 \quad 67$ & $O C \mathrm{~S}$ & 7.846 & 1.030 & 8.454 & 1.024 & 1.1210 & 1.058 & 0.2060 & 1.033 \\
\hline 14868 & OC $S$ & 7.763 & 1.023 & 8.201 & 1.018 & 1.0850 & 1.053 & 0.2130 & 1.030 \\
\hline 14869 & $O C D$ & 7.393 & 1.024 & 7.767 & 1.014 & 0.9775 & 1.085 & 0.2233 & 1.019 \\
\hline 14870 & OC S & 6.895 & 1.012 & 7.134 & 1.024 & 0.9483 & 1.051 & 0.1881 & 1.042 \\
\hline 14871 & OC $\mathrm{s}$ & 6.132 & 1.026 & 6.461 & 1.019 & 0.8512 & 1.060 & 0.1663 & 1.056 \\
\hline 14872 & $O C D$ & 5.333 & 1.024 & 5.615 & 1.023 & 0.7102 & 1.098 & 0.1539 & 1.028 \\
\hline $148 \quad 73$ & OC S & 4.407 & 1.011 & 4.869 & 1.005 & 0.6319 & 1.074 & 0.1115 & 0.957 \\
\hline 14874 & $\mathrm{RB}$ & 3.747 & 0.996 & 4.123 & 1.014 & 0.5204 & 1.048 & 0.0482 & 1.060 \\
\hline 14875 & $\mathrm{RB}$ & 2.871 & 0.999 & 3.260 & 1.023 & 0.3970 & 1.084 & 0.0269 & 0.978 \\
\hline 14876 & $\mathrm{RB}$ & 2.253 & 0.961 & 2.575 & 1.009 & 0.3097 & 1.057 & 0.0142 & 1.008 \\
\hline 14877 & $\mathrm{RB}$ & 1.759 & 0.939 & 2.022 & 1.007 & 0.2309 & 1.060 & 0.0103 & 0.768 \\
\hline 14878 & $\mathrm{RB}$ & 1.464 & 0.925 & 1.659 & 1.041 & 0.1878 & 0.999 & 0.0058 & 0.766 \\
\hline 14879 & $\mathrm{RR}$ & -- & - & 1.665 & 1.079 & -- & -- & - & - \\
\hline $148 \quad 30$ & $\mathrm{RR}$ & - & -- & 1.350 & 1.026 & - & $\cdots$ & -- & - \\
\hline $148 \quad 81$ & $\mathrm{RR}$ & -- & - & 0.828 & 0.734 & -- & - & -- & -- \\
\hline
\end{tabular}


TABLE 6.2 ZPPR-17A: Summary of Radial Reaction Rate Distribution C/E Values ${ }^{\mathrm{a}}$

\begin{tabular}{|c|c|c|c|c|c|c|c|c|c|}
\hline Reaction & Distribution & $\begin{array}{l}\text { Inner } \\
\text { Zone } \\
\end{array}$ & ICA & ICB & ICC & ICD & $\mathrm{OCE}$ & $\mathrm{OCF}$ & RBG \\
\hline${ }^{2}{ }^{y} \mathrm{Pu}(\mathrm{n}, \mathrm{f})$ & $\begin{array}{l}X \text {-axis } \quad Z=5 \mathrm{~cm} \\
X \text {-axis } Z=18 \mathrm{~cm} \\
X \text {-axis } Z=28 \mathrm{~cm}\end{array}$ & $\begin{array}{l}\text { IB } \\
\text { IC } \\
\text { IC }\end{array}$ & $\begin{array}{l}0.962 \\
0.963 \\
0.978\end{array}$ & $\begin{array}{l}0.974 \\
0.965 \\
0.978\end{array}$ & $\begin{array}{l}0.987 \\
0.976 \\
0.985\end{array}$ & $\begin{array}{l}1.010 \\
1.005 \\
1.007\end{array}$ & $\begin{array}{l}1.023 \\
1.025 \\
1.023\end{array}$ & $\begin{array}{l}1.018 \\
1.025 \\
1.017\end{array}$ & $\begin{array}{l}0.974 \\
0.974 \\
0.975\end{array}$ \\
\hline $235 U(n, f)$ & 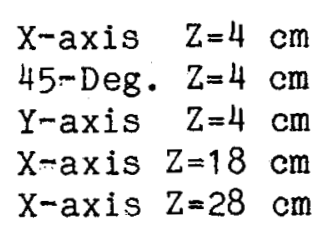 & $\begin{array}{l}\text { IB } \\
\text { IB } \\
\text { IB } \\
\text { IC } \\
\text { IC }\end{array}$ & $\begin{array}{l}0.998 \\
1.006 \\
0.997 \\
0.978 \\
0.971\end{array}$ & $\begin{array}{l}1.009 \\
1.011 \\
1.004 \\
0.983 \\
0.974\end{array}$ & $\begin{array}{l}1.017 \\
1.015 \\
1.015 \\
0.994 \\
0.987\end{array}$ & $\begin{array}{l}1.034 \\
1.026 \\
1.024 \\
1.005 \\
1.003\end{array}$ & $\begin{array}{l}1.020 \\
1.007 \\
1.007 \\
1.018 \\
1.016\end{array}$ & $\begin{array}{l}1.018 \\
1.013 \\
1.004 \\
1.012 \\
1.013\end{array}$ & $\begin{array}{l}1.013 \\
1.026 \\
1.017 \\
1.016 \\
1.015\end{array}$ \\
\hline${ }^{238} U(n, \gamma)$ & $\begin{array}{l}X \text {-axis } \quad Z=5 \mathrm{~cm} \\
Y \text {-axis } \quad Z=5 \mathrm{~cm} \\
X \text {-axis } Z=18 \mathrm{~cm} \\
X \text {-axis } Z=28 \mathrm{~cm}\end{array}$ & $\begin{array}{l}\text { IB } \\
\text { IB } \\
\text { IC } \\
\text { IC }\end{array}$ & $\begin{array}{l}1.018 \\
1.026 \\
1.025 \\
1.013\end{array}$ & $\begin{array}{l}1.023 \\
1.029 \\
1.024 \\
1.029\end{array}$ & $\begin{array}{l}1.032 \\
1.037 \\
1.037 \\
1.032\end{array}$ & $\begin{array}{l}1.042 \\
1.038 \\
1.070 \\
1.055\end{array}$ & $\begin{array}{l}1.066 \\
1.054 \\
1.073 \\
1.071\end{array}$ & $\begin{array}{l}1.071 \\
1.065 \\
1.069 \\
1.066\end{array}$ & $\begin{array}{l}1.062 \\
1.058 \\
1.063 \\
1.080\end{array}$ \\
\hline $238 \mathrm{U}(n, f)$ & $\begin{array}{l}X \text {-axis } Z=5 \mathrm{~cm} \\
Y \text {-axis } Z=5 \mathrm{~cm} \\
X \text {-axis } Z=18 \mathrm{~cm} \\
X \text {-axis } Z=28 \mathrm{~cm}\end{array}$ & $\begin{array}{l}\text { IB } \\
\text { IB } \\
\text { IC } \\
\text { IC }\end{array}$ & $\begin{array}{l}0.955 \\
0.941 \\
0.969 \\
0.979\end{array}$ & $\begin{array}{l}0.985 \\
1.008 \\
0.956 \\
0.975\end{array}$ & $\begin{array}{l}1.010 \\
1.013 \\
0.973 \\
1.008\end{array}$ & $\begin{array}{l}1.036 \\
1.069 \\
1.002 \\
1.008\end{array}$ & $\begin{array}{l}1.021 \\
1.004 \\
1.026 \\
1.034\end{array}$ & $\begin{array}{l}1.030 \\
0.990 \\
1.031 \\
0.990\end{array}$ & $\begin{array}{l}0.954 \\
0.972 \\
0.979 \\
0.955\end{array}$ \\
\hline
\end{tabular}

${ }^{a}$ Results shown are the mean $C / E$ values for four successive matrix positions along the traverse. 
TABLE 6.3 ZPPR-17A: Axial Reaction Rate Distributions in Matrix 148-50 (Core Center)

\begin{tabular}{|c|c|c|c|c|c|c|c|c|c|}
\hline \multirow[b]{2}{*}{ Zone } & \multirow[b]{2}{*}{$z(\mathrm{~mm})$} & \multicolumn{2}{|c|}{${ }^{239} \mathrm{Pu}(\mathrm{n}, \mathrm{f})$} & \multicolumn{2}{|c|}{$235 U(n, f)$} & \multicolumn{2}{|c|}{${ }^{238} U(n, \gamma)$} & \multicolumn{2}{|c|}{${ }^{238} U(n, f)$} \\
\hline & & Exp. & $\mathrm{C} / \mathrm{E}$ & Exp. & $\mathrm{C} / \mathrm{E}$ & Exp. & $\mathrm{C} / \mathrm{E}$ & Exp. & $\mathrm{C} / \mathrm{E}$ \\
\hline IB & 26.2 & 7.049 & 0.958 & 8.060 & 1.006 & 0.9777 & 1.013 & 0.0434 & 0.906 \\
\hline IB & 49.6 & 7.115 & 0.947 & 8.055 & 1.001 & 0.9742 & 1.013 & 0.0417 & 1.015 \\
\hline IB & 77.0 & 6.983 & 0.962 & 8.016 & 0.993 & 0.9636 & 1.019 & 0.0538 & 0.899 \\
\hline IB & 100.4 & 6.912 & 0.966 & 7.889 & 0.994 & 0.9624 & 1.011 & 0.0600 & 0.953 \\
\hline \multirow[t]{3}{*}{ IB } & 127.8 & 6.813 & 0.971 & 7.669 & 0.996 & 0.9414 & 1.018 & 0.0719 & 0.998 \\
\hline & \multirow{2}{*}{\multicolumn{2}{|c|}{$\begin{array}{l}\text { Zone Average } \mathrm{C} / \mathrm{E} \\
\text { Standard Deviation }\end{array}$}} & 0.961 & & 0.988 & & 1.015 & & 0.954 \\
\hline & & & 0.009 & & 0.005 & & 0.003 & & 0.052 \\
\hline IC $S$ & 178.6 & 6.274 & 0.965 & 7.154 & 0.980 & 0.9323 & 1.027 & 0.1337 & 0.975 \\
\hline IC $S$ & 229.4 & 6.399 & 0.960 & 6.947 & 0.974 & 0.8978 & 1.032 & 0.1576 & 1.000 \\
\hline IC $S$ & 280.2 & 6.317 & 0.976 & 6.784 & 0.974 & 0.8933 & 1.012 & 0.1702 & 0.999 \\
\hline IC $S$ & 331.0 & 6.188 & 0.979 & 6.574 & 0.979 & 0.8623 & 1.020 & 0.1691 & 0.997 \\
\hline IC $S$ & 381.8 & 5.827 & 0.989 & 6.286 & 0.976 & 0.8267 & 1.015 & 0.1595 & 0.996 \\
\hline IC $S$ & 432.6 & 5.460 & 0.970 & 5.826 & 0.981 & 0.7715 & 1.014 & 0.1410 & 0.991 \\
\hline \multirow[t]{4}{*}{ IC $S$} & 483.4 & 4.901 & 0.960 & 5.405 & 0.970 & 0.7189 & 1.001 & 0.1183 & 0.925 \\
\hline & & & $\ldots-$ & & $\ldots$ & & $\ldots$ & & \\
\hline & \multirow{2}{*}{\multicolumn{2}{|c|}{$\begin{array}{l}\text { Zone Average } \mathrm{C} / \mathrm{E} \\
\text { Standard Deviation }\end{array}$}} & 0.971 & & 0.976 & & 1.017 & & 0.983 \\
\hline & & & 0.011 & & 0.004 & & 0.010 & & 0.027 \\
\hline$A B$ & 534.2 & 4.604 & 0.954 & 5.115 & 0.970 & 0.6556 & 1.008 & 0.0611 & 0.924 \\
\hline$A B$ & 585.0 & 4.153 & 0.934 & 4.637 & 0.978 & 0.5819 & 1.020 & 0.0364 & 0.871 \\
\hline$A B$ & 635.8 & 3.675 & 0.922 & 4.221 & 0.965 & 0.5176 & 1.007 & 0.0241 & 0.730 \\
\hline$A B$ & 686.6 & 3.291 & 0.901 & 3.756 & 0.972 & 0.4435 & 1.016 & 0.0162 & 0.739 \\
\hline \multirow[t]{4}{*}{$A B$} & 737.4 & 3.070 & 0.884 & 3.450 & 0.997 & 0.4033 & 0.985 & 0.0105 & 0.714 \\
\hline & & & $\ldots-$ & & $\ldots$ & & & & \\
\hline & \multirow{2}{*}{\multicolumn{2}{|c|}{$\begin{array}{l}\text { Zone Average } \mathrm{C} / \mathrm{E} \\
\text { Standard Deviation }\end{array}$}} & 0.919 & & 0.976 & & 1.007 & & 0.796 \\
\hline & & & 0.027 & & 0.012 & & 0.014 & & 0.095 \\
\hline AR & 839.0 & -- & - & 3.741 & 1.126 & -- & - & -- & - \\
\hline$A R$ & 889.8 & - & -- & 3.713 & 1.064 & -- & -- & -- & -- \\
\hline$A R$ & 946.9 & -- & -- & 2.641 & 1.119 & - & - & -- & - \\
\hline$A R$ & 997.7 & -- & -- & 1.937 & 0.988 & -- & -- & - & -- \\
\hline
\end{tabular}


TABLE 6.4 ZPPR-17A: Axial Reaction Rate Distributions in Matrix 148-66 (Outer Core)

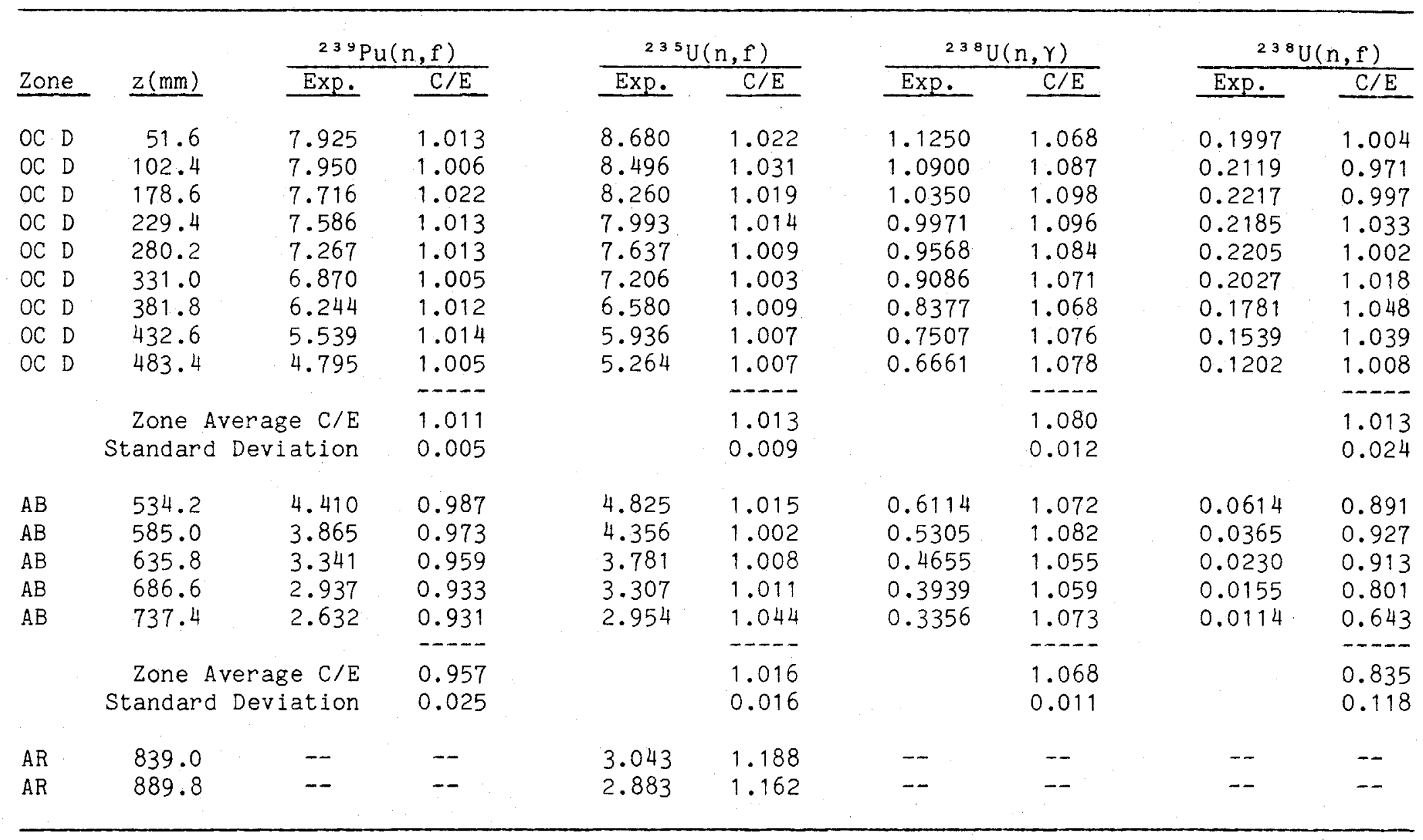


TABLE 6.5 ZPPR-17A: Axial Reaction Rate Distributions in Matrix 148-70 (Outer Core)

\begin{tabular}{|c|c|c|c|c|c|c|c|c|c|}
\hline \multirow[b]{2}{*}{ Zone } & \multirow[b]{2}{*}{$\underline{z}(\mathrm{~mm})$} & \multicolumn{2}{|c|}{$2{ }^{39} \mathrm{Pu}(\mathrm{n}, \mathrm{f})$} & \multicolumn{2}{|c|}{$2{ }^{25} \mathrm{U}(\mathrm{n}, \mathrm{f})$} & \multicolumn{2}{|c|}{${ }^{2{ }^{8}} U(n, \gamma)$} & \multicolumn{2}{|c|}{${ }^{238} U(n, f)$} \\
\hline & & Exp. & $\mathrm{C} / \mathrm{E}$ & Exp. & $\mathrm{C} / \mathrm{E}$ & Exp. & $\mathrm{C} / \mathrm{E}$ & Exp. & $\mathrm{C} / \mathrm{E}$ \\
\hline$O C S$ & 51.6 & 6.895 & 1.012 & 7.134 & 1.024 & 0.9483 & 1.051 & 0.1881 & 1.042 \\
\hline OC S & 102.4 & 6.740 & 1.021 & 7.054 & 1.020 & 0.9360 & 1.049 & 0.1895 & 1.020 \\
\hline$O C S$ & 178.6 & 6.427 & 1.025 & 6.765 & 1.019 & 0.8865 & 1.060 & 0.1764 & 1.050 \\
\hline$O C S$ & 229.4 & 6.163 & 1.021 & 6.483 & 1.016 & 0.8607 & 1.043 & 0.1711 & 1.033 \\
\hline$O C S$ & 280.2 & 5.758 & 1.026 & 6.121 & 1.012 & 0.8056 & 1.048 & 0.1621 & 1.022 \\
\hline OC S & 331.0 & 5.305 & 1.027 & 5.624 & 1.018 & 0.7441 & 1.050 & 0.1448 & 1.048 \\
\hline OC S & 381.8 & 4.809 & 1.020 & 5.089 & 1.021 & 0.6757 & 1.051 & 0.1317 & 1.019 \\
\hline$O C S$ & 432.6 & 4.266 & 1.009 & 4.539 & 1.020 & 0.6011 & 1.056 & 0.1086 & 1.037 \\
\hline \multirow[t]{3}{*}{ OC $S$} & 483.4 & 3.734 & 0.985 & 4.069 & 1.005 & 0.5412 & 1.038 & 0.0875 & 0.974 \\
\hline & \multicolumn{2}{|c|}{ Zone Average $\mathrm{C} / \mathrm{E}$} & 1.016 & & 1.017 & & 1.050 & & 1.027 \\
\hline & \multicolumn{2}{|c|}{ Standard Deviation } & 0.013 & & 0.006 & & 0.006 & & 0.023 \\
\hline$A B$ & 534.2 & 3.344 & 0.989 & 3.710 & 1.002 & 0.4778 & 1.041 & 0.0479 & 0.907 \\
\hline$A B$ & 585.0 & 2.865 & 0.986 & 3.277 & 1.001 & 0.4054 & 1.066 & 0.0270 & 0.902 \\
\hline$A B$ & 635.8 & 2.488 & 0.961 & 2.863 & 0.997 & 0.3521 & 1.046 & 0.0158 & 0.855 \\
\hline$A B$ & 686.6 & 2.174 & 0.936 & 2.448 & 1.015 & 0.2931 & 1.057 & 0.0106 & 0.840 \\
\hline \multirow[t]{3}{*}{$A B$} & 737.4 & 1.953 & 0.928 & 2.189 & 1.042 & 0.2471 & 1.077 & 0.0097 & 0.571 \\
\hline & \multirow{2}{*}{\multicolumn{2}{|c|}{$\begin{array}{l}\text { Zone Average C/E } \\
\text { Standard Deviation }\end{array}$}} & 0060 & & 10 & & 1057 & & 0815 \\
\hline & & & $\begin{array}{l}0.960 \\
0.028\end{array}$ & & $\begin{array}{l}1.011 \\
0.018\end{array}$ & & $\begin{array}{l}1.051 \\
0.015\end{array}$ & & $\begin{array}{l}0.015 \\
0.139\end{array}$ \\
\hline
\end{tabular}




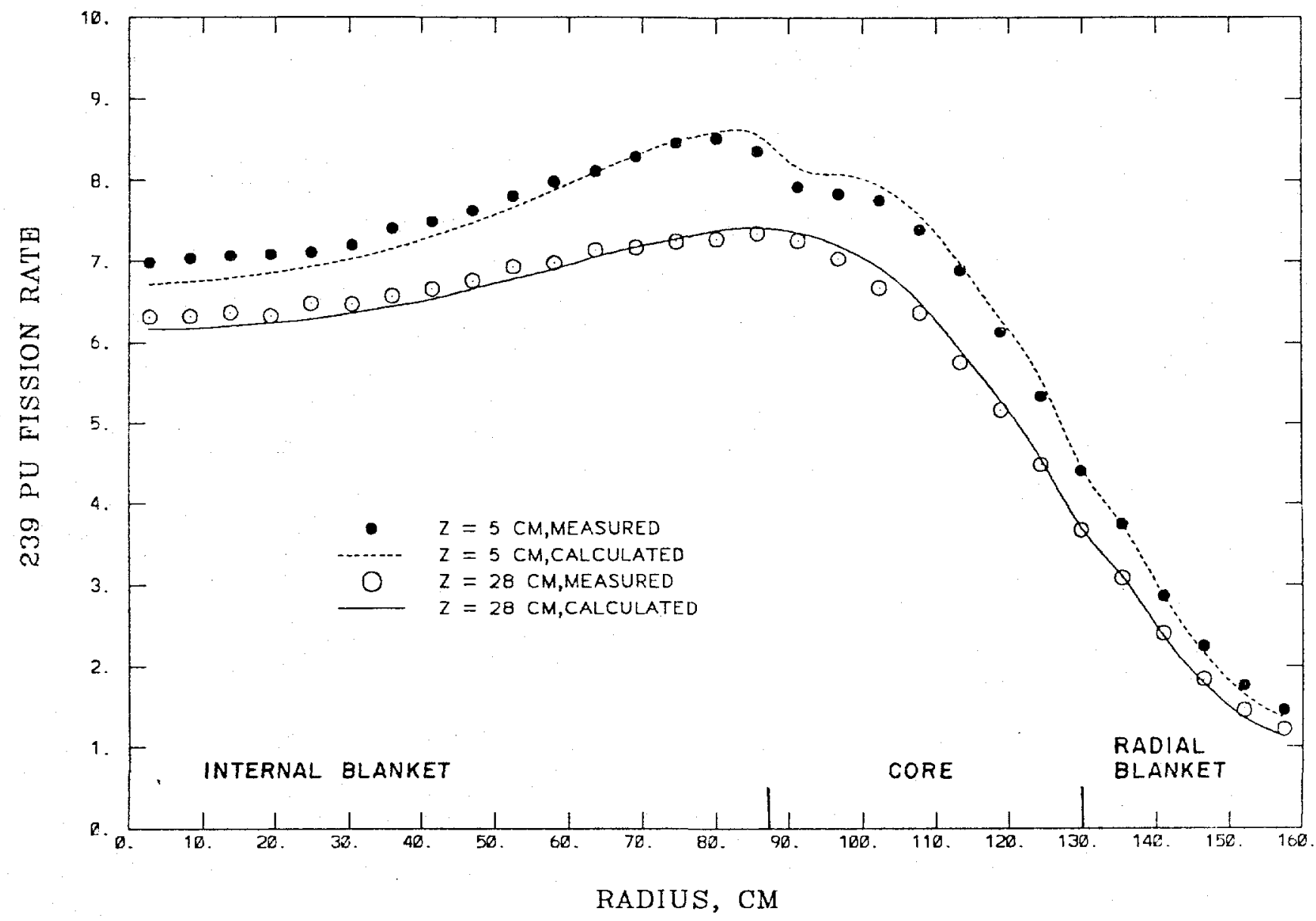

Fig. 6.4 Measured and Calculated Fission Rates in ${ }^{23 y} \mathrm{Pu}$ Along the $\mathrm{x}$-axis of ZPPR-17A. 


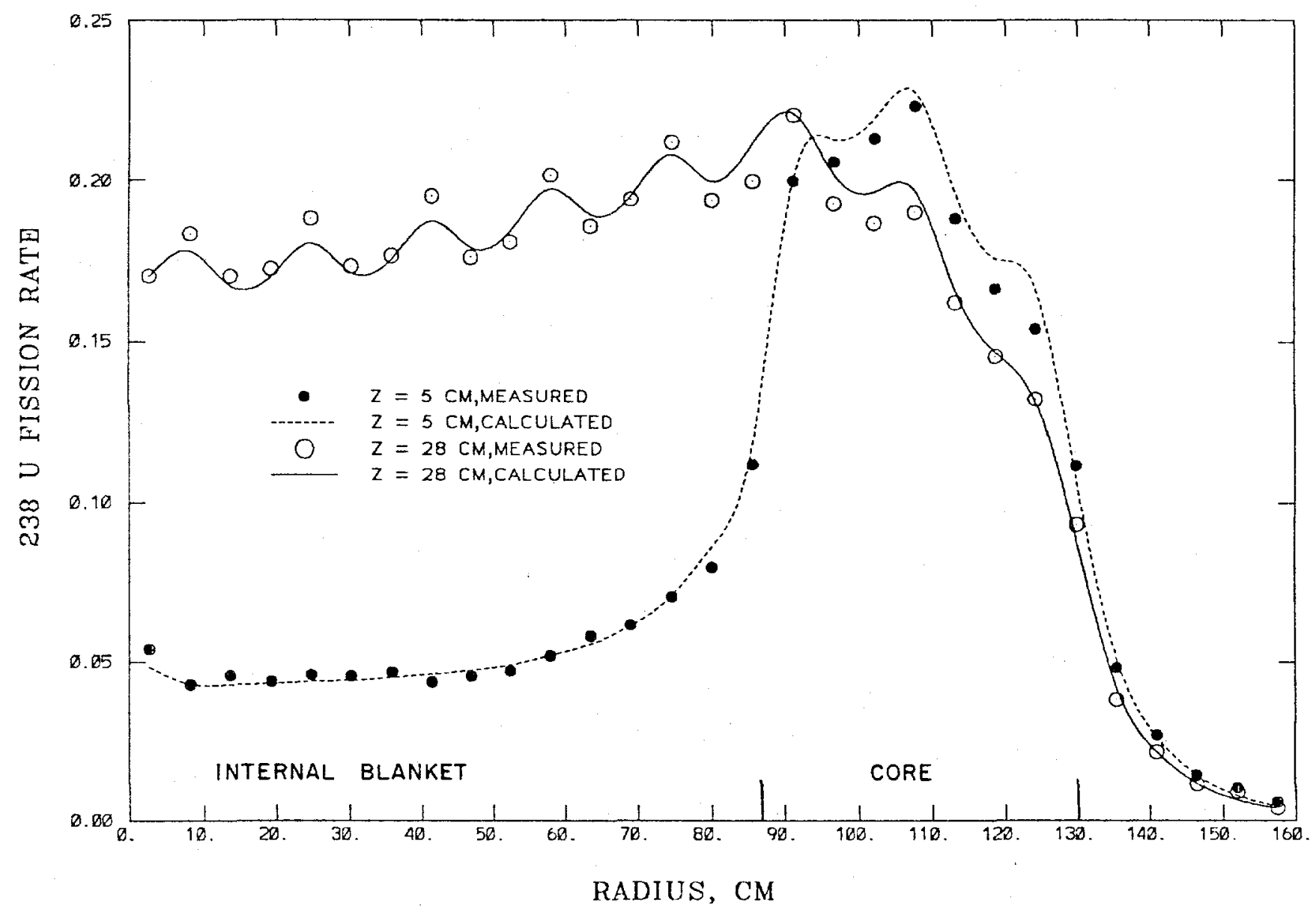

Fig. 6.5 Measured and Calculated Fission Rates in ${ }^{23} \mathrm{U}$ Along the $\mathrm{x}$-axis of ZPPR-17A. 


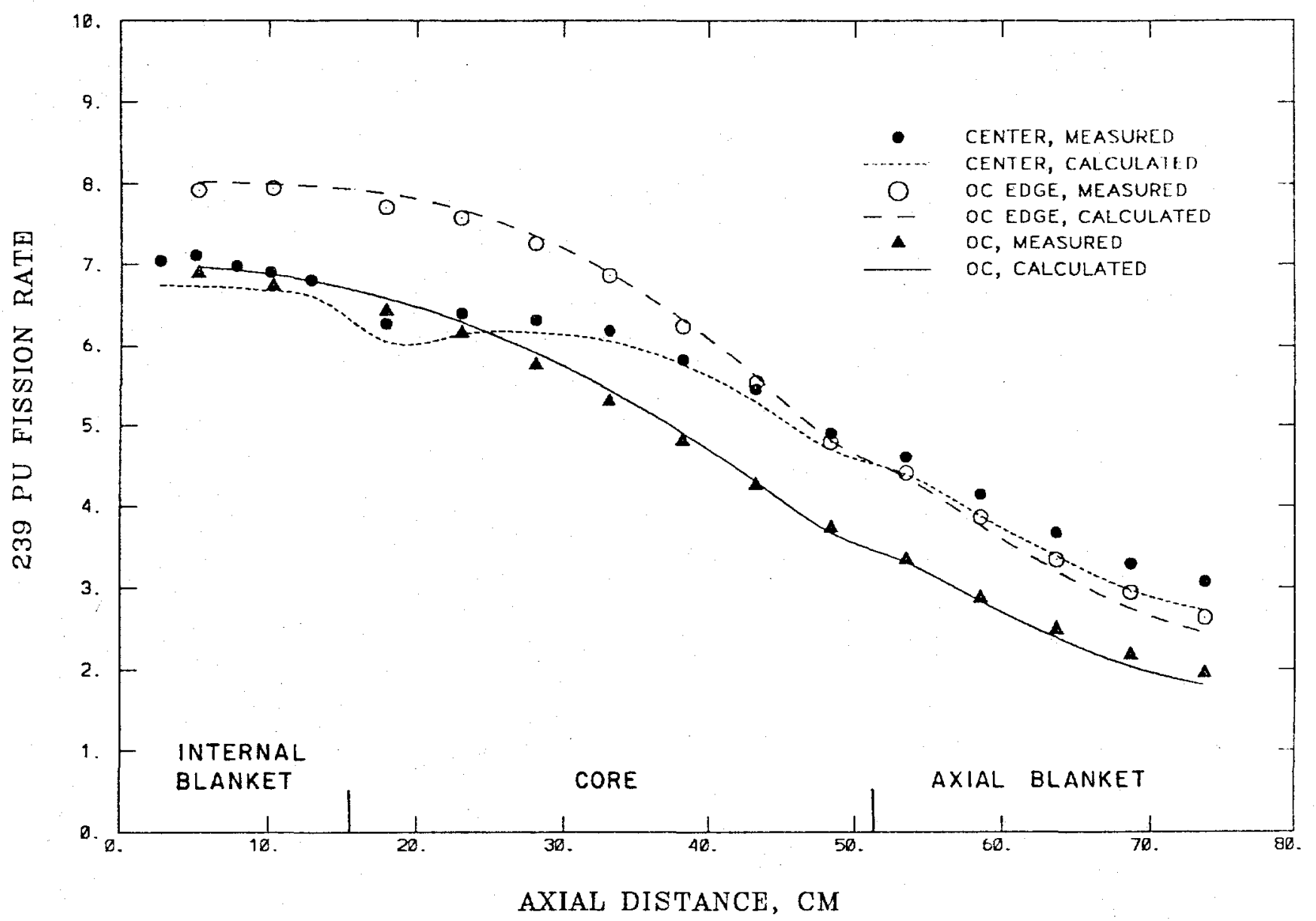

Fig. 6.6. Measured and calculated axial distributions for ${ }^{239} \mathrm{Pu}$ fission in ZPPR-17A. 


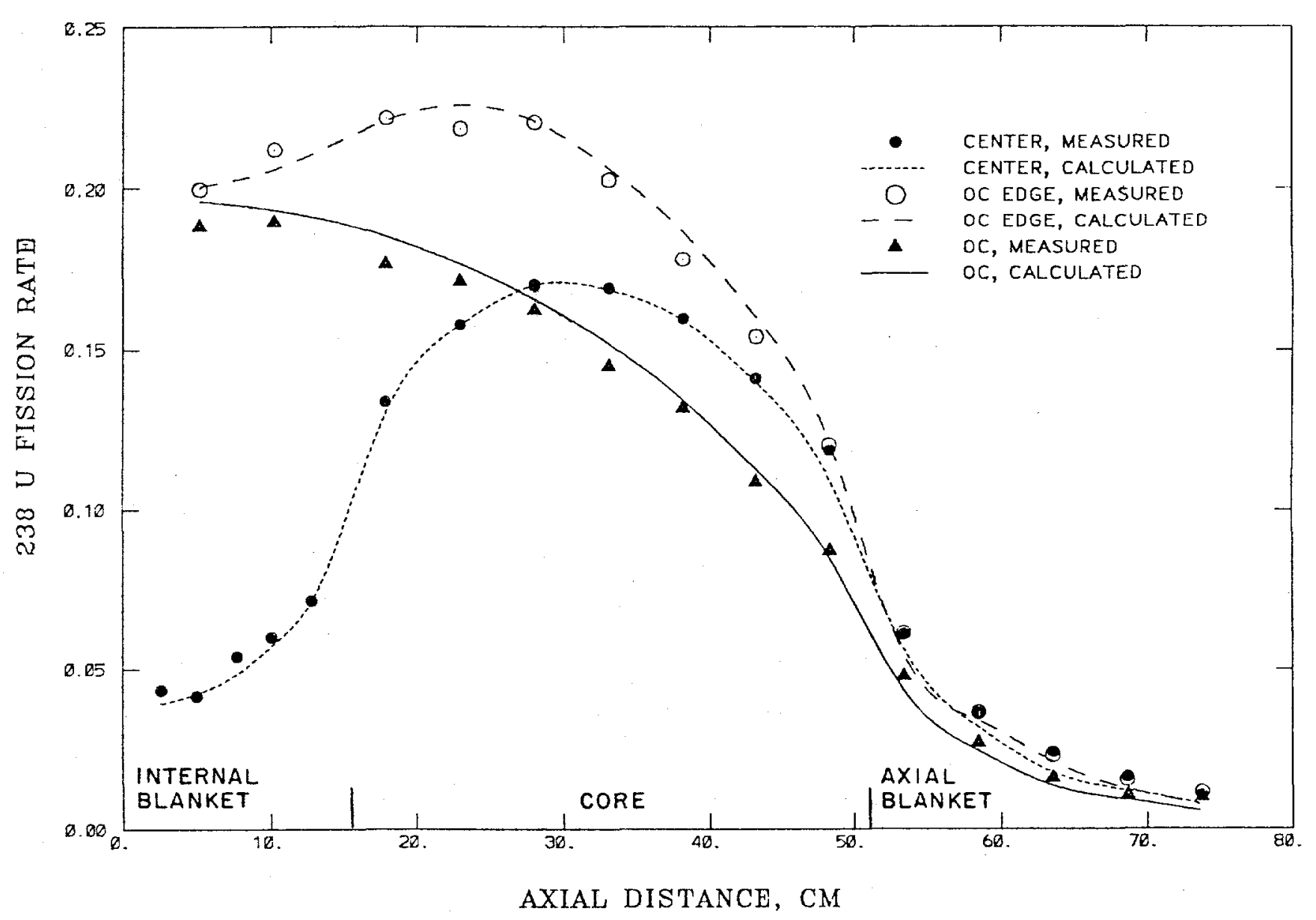

Fig. 6.7. Measured and calculated axial distributions for ${ }^{238} \mathrm{U}$ fission in $\mathrm{ZPPR}-17 \mathrm{~A}$. 


\subsection{Reaction Rate Distributions in ZPPR-17B}

Reaction rate measurements in $\mathrm{ZPPR}-17 \mathrm{~B}$ were made in similar positions to those in $17 \mathrm{~A}$ except that no measurements along the $y$-axis or in symmetric locations were included. Additional traverses for ${ }^{23} \mathrm{U}$ fission at $45^{\circ}$ to the axis, through a control position, were made at $18 \mathrm{~cm}$ and $28 \mathrm{~cm}$ in anticipation of comparisons with ZPPR-17C where a control rod was one-half inserted. For the same reason, one axial traverse was moved to matrix 138-62 (Fig. 6.3) adjacent to the CRP. The axial traverse near the center was moved to matrix 148-51 to be adjacent to the central CRP. Fewer plutonium foils were available for $17 \mathrm{~B}$ and these were used in the axial traverse near to the central CRP and in a number of positions around the center to obtain reaction rate ratio data. Previous analyses have shown that essentially the same information on the accuracy of prediction of fission distributions in the core regions is obtained from ${ }^{235} \mathrm{U}$ foils instead of ${ }^{23 y} \mathrm{Pu}$ foils. This may be verified by examination of the results for ZPPR-17A. Differences between the analysis of ${ }^{235} \mathrm{U}$ and ${ }^{239} \mathrm{Pu}$ are found in the softer spectra of the blanket regions.

The detailed results for ZPPR-17B are found in Reference 5. We show here only the summary of radial reaction analysis in Table 6.6 and the detailed axial distributions in Tables 6.7 to 6.9 . 
TABLE 6.6 ZPPR-17B: Summary of Radial Reaction Rate Distribution Analyses

\begin{tabular}{|c|c|c|c|c|c|c|c|c|c|}
\hline Reaction & Distribution ${ }^{a}$ & $\begin{array}{l}\text { Inner } \\
\text { Zone }\end{array}$ & ICA & ICB & ICC & ICD & OCE & OCF & RBG \\
\hline \multirow{3}{*}{${ }^{23}{ }^{9} \mathrm{Pu}(\mathrm{n}, \mathrm{f})$} & $x$-axis $Z=5 \mathrm{~cm}$ & IB & 1.017 & 1.028 & - & $\ldots$ & $\rightarrow$ & $-\infty$ & -- \\
\hline & $X$-axis $Z=18 \mathrm{~cm}$ & IC & 0.996 & 0.995 & $-\infty$ & $\cdots$ & - & $-\cdots$ & - \\
\hline & $X$-axis $Z=28 \mathrm{~cm}$ & IC & 0.998 & 1.016 & $-r$ & $-\infty$ & $-m$ & $\rightarrow+$ & $-m$ \\
\hline \multirow{6}{*}{$235 U(n, f)$} & $\mathrm{X}$-axis $\mathrm{Z}=5 \mathrm{~cm}$ & IB & 1.039 & 1.048 & 1.053 & 1.074 & 1.061 & 1.068 & 1.066 \\
\hline & 45-Deg. $Z=5 \mathrm{~cm}$ & IB & 1.040 & 1.056 & 1.049 & 1.066 & 1.042 & 1.047 & 1.070 \\
\hline & $\mathrm{X}$-axis $\mathrm{Z}=18 \mathrm{~cm}$ & IC & 1.011 & 1.017 & 1.025 & 1.049 & 1.063 & 1.061 & 1.061 \\
\hline & $45-$ Deg $Z=18 \mathrm{~cm}$ & IC & 1.006 & 1.023 & 1.028 & 1.043 & 1.051 & 1.052 & 1.072 \\
\hline & $X$-axis $Z=28 \mathrm{~cm}$ & IC & 1.011 & 1.025 & 1.028 & 1.039 & 1.059 & 1.060 & 1.065 \\
\hline & $45-$ Deg $Z=28 \mathrm{~cm}$ & IC & 1.010 & 1.008 & 1.023 & 1.035 & 1.054 & 1.046 & 1.065 \\
\hline \multirow[t]{3}{*}{${ }^{238} U(n, \gamma)$} & $X$-axis $Z=5 \mathrm{~cm}$ & IB & 1.066 & 1.076 & 1.088 & 1.093 & 1.109 & 1.119 & 1.105 \\
\hline & $X$-axis $Z=18 \mathrm{~cm}$ & IC & 1.048 & 1.049 & 1.062 & 1.107 & 1.118 & 1.114 & 1.110 \\
\hline & $\mathrm{X}$-axis $\mathrm{Z}=28 \mathrm{~cm}$ & IC & 1.043 & 1.054 & 1.069 & 1.096 & 1.110 & 1.110 & 1.107 \\
\hline \multirow[t]{3}{*}{${ }^{238} \mathrm{U}(\mathrm{n}, \mathrm{f})$} & $X$-axis $Z=5 \mathrm{~cm}$ & IB & 1.032 & 1.048 & 1.088 & 1.142 & 1.051 & 1.066 & 0.983 \\
\hline & $\mathrm{X}$-axis $\mathrm{Z}=18 \mathrm{~cm}$ & IC & 0.980 & 0.996 & 1.008 & 1.052 & 1.062 & 1.067 & 1.028 \\
\hline & $\mathrm{X}$-axis $\mathrm{Z}=28 \mathrm{~cm}$ & IC & 1.016 & 1.006 & 1.024 & 1.052 & 1.064 & 1.055 & 1.007 \\
\hline
\end{tabular}

${ }^{a}$ Results shown are the mean $C / E$ values for groups of successive matrix positions along the traverse. 
TABLE 6.7 ZPPR-17B: Axial Reaction Rate Distributions in Matrix 148-51 (Near Core Center)

\begin{tabular}{|c|c|c|c|c|c|c|c|c|c|}
\hline \multirow[b]{2}{*}{ Zone } & \multirow[b]{2}{*}{$\underline{\mathrm{z}(\mathrm{mm})}$} & \multicolumn{2}{|c|}{${ }^{2{ }^{39} \mathrm{Pu}(n, f)}$} & \multicolumn{2}{|c|}{${ }^{235} U(n, f)$} & \multicolumn{2}{|c|}{$238 \cup(n, \gamma)$} & \multicolumn{2}{|c|}{${ }^{238} \mathrm{U}(\mathrm{n}, \mathrm{f})$} \\
\hline & & Exp. & $\mathrm{C} / \mathrm{E}$ & Exp. & $\mathrm{C} / \mathrm{E}$ & Exp. & $\mathrm{C} / \mathrm{E}$ & Exp. & $\mathrm{C} / \mathrm{E}$ \\
\hline & & & & & & -- & - & & \\
\hline IB & 26.2 & 7.078 & 1.018 & 8.280 & 1.026 & 0.9673 & 1.052 & 0.0435 & 0.969 \\
\hline IB & 49.6 & 7.082 & 1.014 & 8.227 & 1.026 & 0.9658 & 1.050 & 0.0491 & 0.918 \\
\hline IB & 77.0 & 7.023 & 1.014 & 8.109 & 1.027 & 0.9523 & 1.057 & 0.0511 & 1.006 \\
\hline IB & 100.4 & 6.909 & 1.020 & 7.814 & 1.046 & 0.9382 & 1.061 & 0.0560 & 1.075 \\
\hline \multirow[t]{3}{*}{ IB } & 127.8 & 6.800 & 1.017 & 7.751 & 1.021 & 0.9319 & 1.048 & 0.0722 & 1.029 \\
\hline & \multirow{2}{*}{\multicolumn{2}{|c|}{$\begin{array}{r}\text { Zone Average } \mathrm{C} / \mathrm{E} \\
\text { Standard Deviation }\end{array}$}} & 1.017 & & 1.029 & & 1.054 & & 0.999 \\
\hline & & & 0.003 & & 0.010 & & 0.005 & & 0.060 \\
\hline IC $D$ & 178.6 & 6.063 & 0.995 & 7.049 & 0.995 & 0.8894 & 1.034 & 0.1434 & 0.962 \\
\hline IC $D$ & 229.4 & 6.060 & 1.003 & -- & - & 0.8573 & 1.043 & 0.1613 & 0.984 \\
\hline IC $D$ & 280.2 & 6.160 & 0.985 & 6.582 & 1.008 & 0.8414 & 1.041 & 0.1693 & 0.984 \\
\hline IC D & 331.0 & 5.919 & 1.001 & 6.276 & 1.020 & 0.7954 & 1.064 & 0.1733 & 0.962 \\
\hline IC $D$ & 432.6 & 5.235 & 0.992 & 5.652 & 1.003 & 0.7253 & 1.034 & 0.1431 & 0.985 \\
\hline \multirow[t]{3}{*}{ IC D } & 483.4 & 4.725 & 0.981 & 5.270 & 0.995 & 0.6647 & 1.040 & 0.1136 & 0.967 \\
\hline & \multirow{2}{*}{\multicolumn{2}{|c|}{$\begin{array}{r}\text { Zone Average C/E } \\
\text { Standard Deviation }\end{array}$}} & 0.993 & & 1.004 & & 1.043 & & 0.974 \\
\hline & & & 0.009 & & 0.010 & & 0.011 & & 0.011 \\
\hline$A B$ & 534.2 & 4.594 & 0.994 & 5.100 & 1.009 & 0.61 .41 & 1.073 & 0.0536 & 0.981 \\
\hline$A B$ & 585.0 & 4.171 & 0.995 & 4.777 & 1.001 & 0.5723 & 1.045 & 0.0332 & 1.030 \\
\hline$A B$ & 635.8 & 3.878 & 0.961 & 4.336 & 1.009 & 0.4972 & 1.069 & 0.0219 & 1.018 \\
\hline$A B$ & 686.6 & 3.573 & 0.945 & 3.997 & 1.007 & 0.4422 & 1.061 & 0.0147 & 0.913 \\
\hline \multirow[t]{4}{*}{$A B$} & 737.4 & 3.423 & 0.927 & 3.808 & 1.008 & 0.3937 & 1.066 & 0.0111 & 0.740 \\
\hline & & & $\ldots$ & & $\ldots$ & & $\ldots-$ & & $\ldots$ \\
\hline & \multicolumn{2}{|c|}{ Zone Average C/E } & 0.964 & & 1.007 & & 1.063 & & 0.936 \\
\hline & Standard & iation & 0.030 & & 0.003 & & 0.011 & & 0.119 \\
\hline$A R$ & 825.2 & -- & - & 4.008 & 1.129 & -- & - & -- & - \\
\hline$A R$ & 876.0 & -- & - & 3.771 & 1.171 & - & - & -- & -- \\
\hline$A R$ & 933.1 & - & - & 3.008 & 1.152 & - & -- & - & $\cdots$ \\
\hline AR & 983.9 & - & $\sim$ & 2.168 & 1.106 & -- & -- & - & $-\infty$ \\
\hline $\mathrm{AR}$ & 1034.7 & - & $=$ & 1.383 & 0.864 & -- & $=$ & - & - \\
\hline
\end{tabular}


TABLE 6.8 ZPPR-17B: Axial Reaction Rate Distributions in Matrix 138-62 (next to CRP)

\begin{tabular}{|c|c|c|c|c|c|c|c|}
\hline \multirow[b]{2}{*}{ Zone } & \multirow[b]{2}{*}{$z(\mathrm{~mm})$} & \multicolumn{2}{|c|}{$235 \mathrm{U}(\mathrm{n}, \mathrm{f})$} & \multicolumn{2}{|c|}{$2{ }^{38} U(n, \gamma)$} & \multicolumn{2}{|c|}{${ }^{238} U(n, f)$} \\
\hline & & Exp. & $C / E$ & Exp. & $C / E$ & Exp. & $\mathrm{C} / \mathrm{E}$ \\
\hline OC S & 51.6 & 8.954 & 1.040 & 1.1320 & 1.083 & 0.1856 & 0.971 \\
\hline$O C S$ & 102.4 & 8.808 & 1.041 & 1.1000 & 1.098 & 0.1967 & 0.944 \\
\hline$O C S$ & 178.6 & 8.452 & 1.038 & 1.0500 & 1.099 & 0.2032 & 0.999 \\
\hline OC $S$ & 229.4 & 8.016 & 1.047 & 1.0150 & 1.090 & 0.2089 & 1.002 \\
\hline$O C S$ & 280.2 & 7.677 & 1.037 & 0.9642 & 1.087 & 0.2025 & 1.017 \\
\hline OC S & 331.0 & 7.178 & 1.037 & 0.9180 & 1.070 & 0.1905 & 1.006 \\
\hline OC S & 381.8 & 6.595 & 1.040 & 0.8487 & 1.065 & 0.1745 & 0.994 \\
\hline OC S & 432.6 & 5.986 & 1.037 & 0.7622 & 1.073 & 0.1529 & 0.976 \\
\hline \multirow[t]{4}{*}{$O C S$} & 483.4 & 5.338 & 1.040 & 0.6829 & 1.069 & 0.1167 & 0.976 \\
\hline & & & $\cdots$ & & $\cdots$ & & $\cdots-\cdots$ \\
\hline & Zone $A$ & ge $C / E$ & 1.040 & & 1.082 & & 0.987 \\
\hline & Standaro & iation & 0.003 & & 0.013 & & 0.022 \\
\hline $\mathrm{AB}$ & 534.2 & 5.041 & 1.038 & 0.6090 & 1.101 & 0.0572 & 0.938 \\
\hline$A B$ & 585.0 & 4.523 & 1.041 & 0.5327 & 1.108 & 0.0354 & 1.001 \\
\hline$A B$ & 635.8 & 4.008 & 1.042 & 0.4566 & 1.115 & 0.0227 & 1.034 \\
\hline$A B$ & 686.6 & 3.526 & 1.061 & 0.3869 & 1.133 & 0.0142 & 0.960 \\
\hline \multirow[t]{3}{*}{$A B$} & 737.4 & 3.211 & 1.085 & 0.3471 & 1.103 & 0.0102 & 0.798 \\
\hline & Zone $A$ & ge $C / E$ & 1.053 & & 1.112 & & 0.946 \\
\hline & Standaro & iation & 0.020 & & 0.013 & & 0.091 \\
\hline AR & 825.2 & 3.227 & 1.182 & -- & -- & - & - \\
\hline AR & 876.0 & 2.957 & 1.227 & $=$ & - & -- & -- \\
\hline
\end{tabular}


TABLE 6.9 ZPPR-17B: Axial Reaction Rate Distributions in Matrix 148-70 (Outer Core)

\begin{tabular}{|c|c|c|c|c|c|c|c|}
\hline \multirow[b]{2}{*}{ Zone } & \multirow[b]{2}{*}{$\mathrm{z}(\mathrm{mm})$} & \multicolumn{2}{|c|}{$235 U(n, f)$} & \multicolumn{2}{|c|}{${ }^{238} \mathrm{U}(\mathrm{n}, \mathrm{\gamma})$} & \multicolumn{2}{|c|}{${ }^{238} U(n, f)$} \\
\hline & & Exp. & $C / E$ & Exp. & $\mathrm{C} / \mathrm{E}$ & Exp. & $\mathrm{C} / \mathrm{E}$ \\
\hline OC $S$ & 51.6 & $7 \cdot 390$ & 1.062 & 0.9561 & 1.108 & 0.1951 & 1.094 \\
\hline $\mathrm{OC} \mathrm{S}$ & 102.4 & 7.036 & 1.098 & 0.9415 & 1.108 & 0.1949 & 1.079 \\
\hline OC $S$ & 178.6 & 6.935 & 1.066 & 0.9064 & 1.100 & 0.1812 & 1.114 \\
\hline OC $\dot{S}$ & 229.4 & 6.658 & 1.059 & 0.8677 & 1.097 & 0.1759 & 1.096 \\
\hline & 280.2 & 6.246 & 1.061 & 0.8137 & 1.099 & 0.1691 & 1.067 \\
\hline OC $\mathrm{S}$ & 331.0 & 5.767 & 1.062 & 0.7514 & 1.100 & 0.1514 & 1.092 \\
\hline OC $S$ & 381.8 & 5.207 & 1.066 & 0.6885 & 1.091 & 0.1321 & 1.105 \\
\hline OC $\mathrm{s}$ & 432.6 & 4.703 & 1.052 & 0.6153 & 1.090 & 0.1127 & 1.086 \\
\hline \multirow[t]{3}{*}{ OC $S$} & 483.4 & 4.155 & 1.052 & 0.5563 & 1.066 & 0.0897 & 1.032 \\
\hline & \multicolumn{2}{|c|}{ Zone Average $\mathrm{C} / \mathrm{E}$} & 1.064 & & 1.095 & & 1.083 \\
\hline & \multicolumn{2}{|c|}{ Standard Deviation } & 0.014 & & 0.013 & & 0.025 \\
\hline $\mathrm{AB}$ & 534.2 & 3.814 & 1.039 & 0.4753 & 1.092 & 0.0475 & 1.019 \\
\hline $\mathrm{AB}$ & 585.0 & 3.304 & 1.061 & 0.4129 & 1.091 & 0.0306 & 0.883 \\
\hline$A B$ & 635.8 & 2.917 & 1.050 & 0.3560 & 1.080 & 0.0178 & 0.845 \\
\hline$A B$ & 686.6 & 2.549 & 1.050 & 0.2976 & 1.090 & 0.0119 & 0.836 \\
\hline \multirow[t]{3}{*}{$A B$} & 737.4 & 2.275 & 1.084 & 0.2597 & 1.076 & 0.0088 & 0.702 \\
\hline & \multicolumn{2}{|c|}{ Zone Average $\mathrm{C} / \mathrm{E}$} & 1.057 & & 1.086 & & 0.857 \\
\hline & \multicolumn{2}{|c|}{ Standard Deviation } & 0.017 & & 0.007 & & 0.114 \\
\hline
\end{tabular}




\subsection{Reaction Rate Distributions in ZPPR-17C}

Measurements in $2 P P R-17 \mathrm{C}$ were made in the same locations as in 17B but duplicated in both halves of the reactor. The intent was to compare measured values and the accuracy of calculations both for the axial shapes adjacent to the half-inserted control rods and the perturbations in the radial distributions. The plutonium fission rates adjacent to the central control position were measured in both halves of the core.

As for ZPPR-17B, we show here only a summary of the analysis of radial distributions in Table 6.10 . Detailed axial distributions are given in Tables 6.11 to 6.16 . The control rods were inserted in reactor half two.

The measured axial ${ }^{235} \mathrm{U}$ fission distributions adjacent to the central control position in both $\mathrm{ZPPR}-17 \mathrm{~B}$ and $17 \mathrm{C}$ are shown in Fig. 6.8. The measured distributions in the two cores adjacent to an outer control position are shown in Fig. 6.9 .

Measured ${ }^{235} \mathrm{U}$ fission radial distributions at the six axial heights along the $\mathrm{x}$-axis of ZPPR-17C are shown in Fig. 6.10.

Reaction rate results for $Z P P R-17 C$ are given in greater detail in Reference 5 . 
TABLE 6.10 ZPPR-17C: Summary of Radial Reaction Rate Distribution Analysis

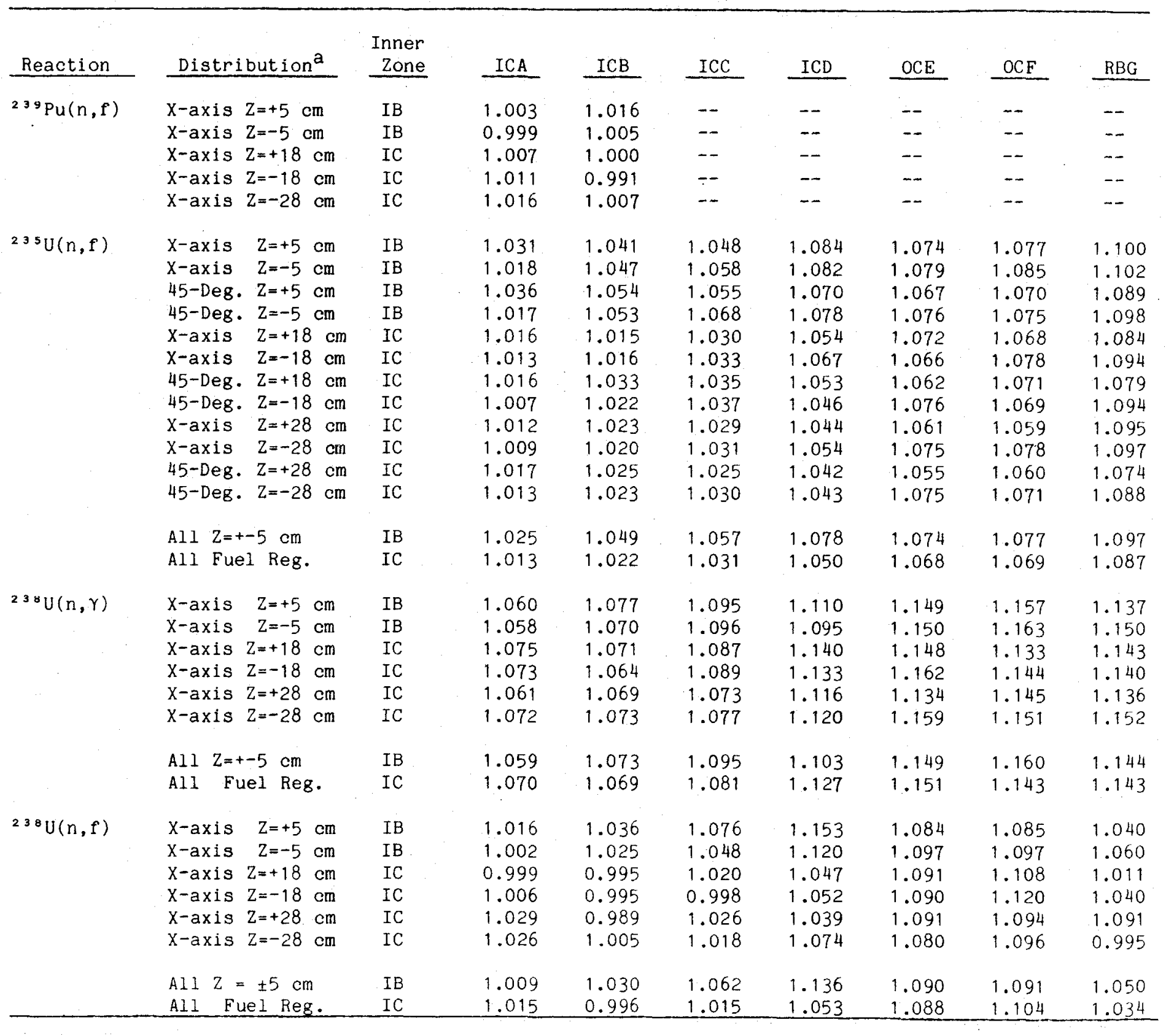


TABLE 6.11 ZPPR-17C: Axial Reaction Rate Distributions in Matrix 148-51 (near Core Center)

\begin{tabular}{|c|c|c|c|c|c|c|c|c|c|}
\hline \multirow[b]{2}{*}{ Zone } & \multirow[b]{2}{*}{$\underline{z(\mathrm{~mm})}$} & \multicolumn{2}{|c|}{${ }^{239} \mathrm{Pu}(\mathrm{n}, \mathrm{f})$} & \multicolumn{2}{|c|}{${ }^{235} U(n, f)$} & \multicolumn{2}{|c|}{$238 \mathrm{U}(\mathrm{n}, \mathrm{Y})$} & \multicolumn{2}{|c|}{${ }^{238} U(n, f)$} \\
\hline & & Exp. & $\mathrm{C} / \mathrm{E}$ & Exp. & $\mathrm{C} / \mathrm{E}$ & Exp. & $\mathrm{C} / \mathrm{E}$ & Exp. & $C / E$ \\
\hline IB & 26.2 & 5.625 & 0.995 & 6.648 & 1.008 & 0.8000 & 1.046 & 0.0390 & 1.013 \\
\hline IB & 49.6 & 5.924 & 1.005 & 6.990 & 1.018 & 0.8319 & 1.062 & 0.0444 & 0.996 \\
\hline IB & 77.0 & 6.267 & 1.011 & 7.211 & 1.032 & 0.8680 & 1.069 & 0.0534 & 0.986 \\
\hline IB & 100.4 & 6.482 & 1.001 & 7.371 & 1.022 & 0.8921 & 1.058 & 0.0604 & 1.051 \\
\hline \multirow[t]{3}{*}{ IB } & 127.8 & 6.596 & 1.005 & 7.428 & 1.020 & 0.8985 & 1.064 & 0.0775 & 1.033 \\
\hline & \multirow{2}{*}{\multicolumn{2}{|c|}{$\begin{array}{l}\text { Zone Average } \mathrm{C} / \mathrm{E} \\
\text { Standard Deviation }\end{array}$}} & 1.003 & & 1.020 & & 1.059 & & 1.016 \\
\hline & & & 0.006 & & 0.009 & & 0.009 & & 0.027 \\
\hline IC D & 178.6 & 6.332 & 1.009 & 7.171 & 1.013 & 0.9163 & 1.063 & 0.1574 & 0.967 \\
\hline IC $D$ & 229.4 & 6.609 & 1.010 & 7.106 & 1.030 & 0.9108 & 1.075 & 0.1865 & 0.993 \\
\hline IC $D$ & 280.2 & 6.846 & 1.001 & 7.232 & 1.022 & 0.9209 & 1.073 & 0.1978 & 0.979 \\
\hline & 331.0 & 6.725 & 1.029 & 7.026 & 1.045 & 0.9013 & 1.091 & 0.2028 & 0.988 \\
\hline & 381.8 & 6.494 & 1.010 & 6.758 & 1.039 & 0.8855 & 1.059 & 0.1876 & 1.003 \\
\hline IC $D$ & 432.6 & 6.064 & 1.001 & 6.458 & 1.018 & 0.8302 & 1.058 & 0.1684 & 1.015 \\
\hline \multirow[t]{3}{*}{ IC D } & 483.4 & 5.555 & 0.987 & 6.029 & 1.017 & 0.7786 & 1.054 & 0.1386 & 0.960 \\
\hline & \multirow{2}{*}{\multicolumn{2}{|c|}{$\begin{array}{l}\text { Zone Average } C / E \\
\text { Standard Deviation }\end{array}$}} & 1.007 & & 1.026 & & 1.068 & & 0.983 \\
\hline & & & 0.013 & & 0.012 & & 0.013 & & 0.021 \\
\hline$A B$ & 534.2 & -- & - & 5.832 & 1.027 & 0.7209 & 1.091 & 0.0639 & 1.051 \\
\hline$A B$ & 585.0 & - & -- & 5.405 & 1.044 & 0.6618 & 1.093 & 0.0414 & 0.960 \\
\hline$A B$ & 635.8 & -- & - & 5.008 & 1.045 & 0.5845 & 1.115 & 0.0279 & 0.962 \\
\hline$A B$ & 686.6 & -- & -- & 4.577 & 1.077 & 0.5135 & 1.140 & 0.0190 & 0.838 \\
\hline \multirow[t]{3}{*}{$A B$} & 737.4 & -- & -- & 4.222 & 1.120 & 0.4592 & 1.146 & 0.0107 & 0.945 \\
\hline & \multirow{2}{*}{\multicolumn{2}{|c|}{$\begin{array}{l}\text { Zone Average C/E } \\
\text { Standard Deviation }\end{array}$}} & -- & & 1.063 & & 1.117 & & 0.951 \\
\hline & & & - & & 0.037 & & 0.026 & & 0.076 \\
\hline$A R$ & 825.2 & - & - & 4.668 & 1.099 & -- & - & - & -- \\
\hline$A R$ & 876.0 & -- & -- & 4.350 & 1.155 & - & -- & -- & -- \\
\hline$A R$ & 933.1 & -- & - & 3.453 & 1.251 & - & - & - & - \\
\hline$A R$ & 983.9 & -- & -- & 2.550 & 1.120 & - & -- & -- & - \\
\hline AR & 1034.7 & - & - & 1.597 & 0.861 & -- & - & - & - \\
\hline
\end{tabular}


TABLE 6.12 ZPPR-17C: Axial Reaction Rate Distributions in Matrix 248-51 (near Core Center)

\begin{tabular}{|c|c|c|c|c|c|c|c|c|c|}
\hline \multirow[b]{2}{*}{ Zone } & \multirow[b]{2}{*}{$z(\mathrm{~mm})$} & \multicolumn{2}{|c|}{${ }^{239} \mathrm{Pu}(\mathrm{n}, \mathrm{f})$} & \multicolumn{2}{|c|}{${ }^{235} \mathrm{U}(\mathrm{n}, \mathrm{f})$} & \multicolumn{2}{|c|}{${ }^{238} U(n, \gamma)$} & \multicolumn{2}{|c|}{${ }^{238} \mathrm{U}(\mathrm{n}, \mathrm{f})$} \\
\hline & & Exp. & $C / E$ & Exp. & $C / E$ & Exp. & $C / E$ & Exp. & $\mathrm{C} / \mathrm{E}$ \\
\hline $\mathrm{AR}$ & 1034.7 & -- & -- & 0.900 & 0.779 & - & - & - & -- \\
\hline AR & 983.9 & -- & -- & 1.356 & 1.063 & - & - & -. & -- \\
\hline AR & 933.1 & - & - & 1.787 & 1.206 & - & - & -- & -. \\
\hline$A R$ & 876.0 & - & -- & 2.043 & 1.152 & -- & -- & -- & - \\
\hline $\mathrm{AR}$ & 825.2 & - & -- & 2.022 & 1.052 & -- & -- & - & -- \\
\hline$A B$ & 737.4 & 1.306 & 0.964 & 1.474 & 1.140 & 0.1871 & 1.061 & 0.0055 & 0.851 \\
\hline$A B$ & 686.6 & 1.415 & 0.964 & 1.642 & 1.060 & 0.2062 & 1.071 & 0.0096 & 0.806 \\
\hline$A B$ & 635.8 & 1.594 & 0.984 & 1.885 & 1.022 & 0.2448 & 1.037 & 0.0121 & 1.108 \\
\hline$A B$ & 585.0 & 1.860 & 1.015 & 2.138 & 1.053 & 0.2827 & 1.059 & 0.0194 & 1.069 \\
\hline \multirow[t]{3}{*}{$A B$} & 534.2 & 2.161 & 1.032 & 2.463 & 1.041 & 0.3226 & 1.062 & 0.0331 & 1.081 \\
\hline & \multirow{2}{*}{\multicolumn{2}{|c|}{$\begin{array}{l}\text { Zone Average C/E } \\
\text { Standard Deviation }\end{array}$}} & 0.992 & & 1.063 & & 1.058 & & 0.983 \\
\hline & & & 0.031 & & 0.045 & & 0.013 & & 0.143 \\
\hline IC $\mathrm{D}$ & 483.4 & 2.491 & 1.007 & 2.717 & 1.024 & 0.3494 & 1.065 & 0.0709 & 0.986 \\
\hline IC $D$ & 432.6 & 2.879 & 1.008 & 3.034 & 1.029 & 0.3929 & 1.055 & 0.0907 & 1.014 \\
\hline IC D & 381.8 & 3.193 & 1.012 & 3.386 & 1.016 & 0.4341 & 1.049 & 0.1032 & 1.009 \\
\hline IC $\mathrm{D}$ & 331.0 & 3.414 & 1.025 & 3.631 & 1.019 & 0.4585 & 1.069 & 0.1103 & 1.030 \\
\hline IC D & 280.2 & 3.491 & 1.020 & 3.770 & 1.021 & 0.4827 & 1.057 & 0.1117 & 1.003 \\
\hline IC $\mathrm{D}$ & 229.4 & 3.552 & 1.004 & 3.863 & 1.019 & 0.4949 & 1.059 & 0.1088 & 1.004 \\
\hline \multirow[t]{3}{*}{ IC D } & 178.6 & 3.525 & 1.000 & 4.107 & 0.989 & 0.5175 & 1.053 & 0.0908 & 1.013 \\
\hline & \multirow{2}{*}{\multicolumn{2}{|c|}{$\begin{array}{l}\text { Zone Average C/E } \\
\text { Standard Deviation }\end{array}$}} & 1.011 & & 1.017 & & 1.058 & & 1.006 \\
\hline & & & 0.009 & & 0.013 & & 0.007 & & 0.020 \\
\hline IB & 127.8 & 3.909 & 1.002 & 4.592 & 1.015 & 0.5681 & 1.047 & 0.0500 & 1.041 \\
\hline IB & 100.4 & 4.020 & 1.000 & 4.833 & 1.010 & 0.5946 & 1.041 & 0.0421 & 1.022 \\
\hline IB & 77.0 & 4.170 & 0.988 & 5.025 & 1.009 & 0.6173 & 1.037 & 0.0364 & 1.034 \\
\hline IB & 49.6 & 4.375 & 1.006 & 5.314 & 1.016 & 0.6497 & 1.048 & 0.0349 & 0.998 \\
\hline \multirow[t]{3}{*}{ IB } & 26.2 & 4.735 & 0.992 & 5.671 & 1.024 & 0.6850 & 1.058 & 0.0334 & 1.032 \\
\hline & \multirow{2}{*}{\multicolumn{2}{|c|}{$\begin{array}{l}\text { Zone Average C/E } \\
\text { Standard Deviation }\end{array}$}} & 0.998 & & 1.015 & & 1.046 & & 1.025 \\
\hline & & & 0.007 & & 0.006 & & 0.007 & & 0.017 \\
\hline
\end{tabular}


TABLE 6.13 ZPPR-17C: Axial Reaction Rate Distributions in Matrix 138-62 (next to CRP)

\begin{tabular}{|c|c|c|c|c|c|c|c|}
\hline \multirow[b]{2}{*}{ Zone } & \multirow[b]{2}{*}{$\underline{\mathrm{z}(\mathrm{mm})}$} & \multicolumn{2}{|c|}{$235 \mathrm{U}(\mathrm{n}, \mathrm{f})$} & \multicolumn{2}{|c|}{${ }^{238} \mathrm{U}(\mathrm{n}, \mathrm{Y})$} & \multicolumn{2}{|c|}{$2{ }^{38} \mathrm{U}(\mathrm{n}, \mathrm{f})$} \\
\hline & & Exp. & $C / E$ & Exp. & $C / E$ & Exp. & $C / E$ \\
\hline$O C D$ & 51.6 & 7.370 & 1.045 & 0.9295 & 1.144 & 0.1691 & 1.018 \\
\hline$O C D$ & 102.4 & 7.904 & 1.051 & 0.9882 & 1.138 & 0.1873 & 1.013 \\
\hline$O C D$ & 178.6 & 8.130 & 1.054 & 1.0170 & 1.129 & 0.2159 & 1.021 \\
\hline$O C D$ & 229.4 & 8.099 & 1.044 & 1.0200 & 1.108 & 0.2271 & 1.031 \\
\hline$O C D$ & 280.2 & 7.933 & 1.042 & 1.0070 & 1.095 & 0.2322 & 1.029 \\
\hline$O C D$ & 331.0 & 7.596 & 1.053 & 0.9661 & 1.106 & 0.2160 & 1.082 \\
\hline$O C D$ & 381.8 & 7.094 & 1.049 & 0.9153 & 1.084 & 0.2050 & 1.009 \\
\hline$O C D$ & 432.6 & 6.516 & 1.039 & 0.8277 & 1.091 & 0.1760 & 1.027 \\
\hline \multirow[t]{4}{*}{$O C D$} & 483.4 & 5.912 & 1.034 & 0.7510 & 1.087 & 0.1365 & 1.005 \\
\hline & & & $-\infty$ & & $\cdots$ & & $\cdots$ \\
\hline & Zone A & ge $C / E$ & 1.046 & & 1.109 & & 1.026 \\
\hline & Standaro & iation & 0.007 & & 0.023 & & 0.023 \\
\hline$A B$ & 534.2 & 5.514 & 1.041 & 0.6643 & 1.139 & 0.0648 & 1.050 \\
\hline$A B$ & 585.0 & 5.005 & 1.050 & 0.5954 & 1.139 & 0.0371 & 1.090 \\
\hline$A B$ & 635.8 & 4.453 & 1.065 & 0.5171 & 1.150 & 0.0256 & 1.062 \\
\hline $\mathrm{AB}$ & 686.6 & 3.984 & 1.092 & 0.4474 & 1.168 & 0.0172 & 0.918 \\
\hline \multirow[t]{4}{*}{$A B$} & 737.4 & 3.602 & 1.130 & 0.3864 & 1.190 & 0.0123 & 0.803 \\
\hline & & & $\cdots$ & & 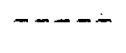 & & \\
\hline & Zone A & ge $C / E$ & 1.076 & & 1.157 & & 0.984 \\
\hline & Standard & iation & 0.036 & & 0.022 & & 0.122 \\
\hline AR & 825.1 & 3.601 & 1.152 & - & -- & -- & -- \\
\hline AR & 875.9 & 3.378 & 1.174 & -- & -- & -- & -- \\
\hline
\end{tabular}


TABLE 6.14 ZPPR-17C: Axial Reaction Rate Distributions in Matrix 238-62 (next to CR)

\begin{tabular}{|c|c|c|c|c|c|c|c|}
\hline \multirow[b]{2}{*}{ Zone } & \multirow[b]{2}{*}{$\underline{z(m m)}$} & \multicolumn{2}{|c|}{$235 U(n, f)$} & \multicolumn{2}{|c|}{$2{ }^{38} \mathrm{U}(n, \gamma)$} & \multicolumn{2}{|c|}{${ }^{238} U(n, f)$} \\
\hline & & Exp. & $C / E$ & Exp. & $C / E$ & Exp. & $\mathrm{C} / \mathrm{E}$ \\
\hline AR & 875.9 & 1.290 & 1.093 & -- & -- & -- & -- \\
\hline AR & 825.1 & 1.232 & 1.073 & - & -- & -- & - \\
\hline $\mathrm{AB}$ & 737.4 & 1.058 & 1.088 & 0.1546 & 1.067 & 0.0064 & 0.685 \\
\hline$A B$ & 686.6 & 1.193 & 1.059 & 0.1707 & 1.111 & 0.0108 & 0.687 \\
\hline$A B$ & 635.8 & 1.410 & 1.040 & 0.2090 & 1.074 & 0.0144 & 0.912 \\
\hline$A B$ & 585.0 & 1.690 & 1.055 & 0.2522 & 1.081 & 0.0198 & 1.026 \\
\hline \multirow[t]{3}{*}{$A B$} & 534.2 & 2.034 & 1.038 & 0.2969 & 1.079 & 0.0326 & 1.075 \\
\hline & \multirow{2}{*}{\multicolumn{2}{|c|}{ Zone Average $C / E$}} & 1.056 & & 1.082 & & 0.877 \\
\hline & & & 0.020 & & 0.017 & & 0.184 \\
\hline$O C D$ & 483.4 & 2.337 & 1.057 & 0.3290 & 1.115 & 0.0681 & 1.032 \\
\hline$O C D$ & 432.6 & 2.760 & 1.055 & 0.3844 & 1.110 & 0.0945 & 1.013 \\
\hline$O C D$ & 381.8 & 3.146 & 1.063 & 0.4335 & 1.124 & 0.1109 & 1.026 \\
\hline$O C D$ & 331.0 & 3.471 & 1.078 & 0.4829 & 1.125 & 0.1241 & 1.072 \\
\hline$O C D$ & 280.2 & 3.771 & 1.080 & 0.5294 & 1.117 & 0.1308 & 1.076 \\
\hline$O C D$ & 229.4 & 4.067 & 1.076 & 0.5637 & 1.133 & 0.1401 & 1.020 \\
\hline$O C D$ & 178.6 & 4.349 & 1.074 & 0.6072 & 1.132 & 0.1379 & 1.021 \\
\hline$O C D$ & 102.4 & 4.869 & 1.058 & 0.6789 & 1.132 & 0.1333 & 1.017 \\
\hline \multirow[t]{3}{*}{$O C D$} & 51.6 & 5.356 & 1.073 & 0.7486 & 1.126 & 0.1411 & 0.992 \\
\hline & & & 10 & & 12 & & 1030 \\
\hline & \multicolumn{2}{|c|}{$\begin{array}{l}\text { Zone Average C/E } \\
\text { Standard Deviation }\end{array}$} & $\begin{array}{l}1.068 \\
0.010\end{array}$ & & $\begin{array}{l}1.124 \\
0.008\end{array}$ & & $\begin{array}{l}1.030 \\
0.027\end{array}$ \\
\hline
\end{tabular}


TABLE 6.15 ZPPR-17C: Axial Reaction Rate Distributions in Matrix 148-70 (Outer Core)

\begin{tabular}{|c|c|c|c|c|c|c|c|}
\hline \multirow[b]{2}{*}{ Zone } & \multirow[b]{2}{*}{$\underline{\mathrm{z}(\mathrm{mm})}$} & \multicolumn{2}{|c|}{${ }^{235} U(n, f)$} & \multicolumn{2}{|c|}{$238 \mathrm{U}(\mathrm{n}, \mathrm{Y})$} & \multicolumn{2}{|c|}{$238 \mathrm{U}(n, f)$} \\
\hline & & Exp. & $\mathrm{C} / \mathrm{E}$ & Exp. & $C / E$ & Exp. & $\mathrm{C} / \mathrm{E}$ \\
\hline$O C D$ & 51.6 & 7.074 & 1.088 & 0.8810 & 1.163 & 0.2270 & 1.085 \\
\hline & 102.4 & 7.166 & 1.090 & 0.8865 & 1.172 & 0.2301 & 1.087 \\
\hline & 178.6 & 7.193 & 1.075 & 0.8889 & 1.157 & 0.2229 & 1.113 \\
\hline & 229.4 & 6.971 & 1.074 & 0.8701 & 1.144 & 0.2184 & 1.102 \\
\hline$O C D$ & 280.2 & 6.717 & 1.068 & 0.8365 & 1.141 & 0.2101 & 1.096 \\
\hline$O C D$ & 331.0 & 6.236 & 1.091 & 0.7828 & 1.158 & 0.1957 & 1.105 \\
\hline$O C D$ & 381.8 & 5.700 & 1.088 & 0.7155 & 1.155 & 0.1796 & 1.061 \\
\hline$O C D$ & 432.6 & 5.113 & 1.082 & 0.6444 & 1.147 & 0.1482 & 1.099 \\
\hline \multirow[t]{4}{*}{$O C D$} & 483.4 & 4.531 & 1.077 & 0.5715 & 1.146 & 0.1087 & 1.107 \\
\hline & & & $-\cdots$ & & $-\cdots$ & & $\cdots-\cdots$ \\
\hline & Zone & ge $C / E$ & 1.081 & & 1.154 & & 1.095 \\
\hline & Standarc & iation & 0.008 & & 0.010 & & 0.016 \\
\hline$A B$ & 534.2 & 4.150 & 1.046 & 0.5286 & 1.105 & 0.0573 & 1.066 \\
\hline $\mathrm{AB}$ & 585.0 & 3.704 & 1.048 & 0.4601 & 1.121 & 0.0315 & 1.091 \\
\hline$A B$ & 635.8 & 3.245 & 1.052 & 0.4029 & 1.102 & 0.0221 & 0.986 \\
\hline$A B$ & 686.6 & 2.828 & 1.085 & 0.3418 & 1.124 & 0.0117 & 1.072 \\
\hline \multirow[t]{3}{*}{$A B$} & 737.4 & 2.549 & 1.109 & 0.2933 & 1.135 & 0.0098 & 0.783 \\
\hline & & $\sigma e \mathrm{C} / \mathrm{F}$ & $-1-1$ & & 1117 & & 1000 \\
\hline & $\begin{array}{l}\text { Zone } \\
\text { Standar }\end{array}$ & $\begin{array}{l}\text { ge } \mathrm{C} / \mathrm{E} \\
\text { iation }\end{array}$ & $\begin{array}{l}1.068 \\
0.028\end{array}$ & & $\begin{array}{l}1.117 \\
0.014\end{array}$ & & $\begin{array}{l}1.000 \\
0.128\end{array}$ \\
\hline
\end{tabular}


TABLE 6.16 ZPPR-17C: Axial Reaction Rate Distributions in Matrix 248-70 (Outer Core)

\begin{tabular}{|c|c|c|c|c|c|c|c|}
\hline \multirow[b]{2}{*}{ Zone } & \multirow[b]{2}{*}{$\underline{z(m m)}$} & \multicolumn{2}{|c|}{$235 \mathrm{U}(n, f)$} & \multicolumn{2}{|c|}{${ }^{238} U(n, \gamma)$} & \multicolumn{2}{|c|}{${ }^{238} U(n, f)$} \\
\hline & & Exp. & $\mathrm{C} / \mathrm{E}$ & Exp. & $C / E$ & Exp. & $C / E$ \\
\hline$A B$ & 737.4 & 1.406 & 1.100 & 0.1657 & 1.118 & 0.0078 & 0.619 \\
\hline$A B$ & 686.6 & 1.597 & 1.077 & 0.1905 & 1.149 & 0.0084 & 0.953 \\
\hline$A B$ & 635.8 & 1.876 & 1.050 & 0.2342 & 1.108 & 0.0137 & 1.017 \\
\hline$A B$ & 585.0 & 2.169 & 1.068 & 0.2739 & 1.133 & 0.0211 & 1.051 \\
\hline \multirow[t]{3}{*}{$A B$} & 534.2 & 2.535 & 1.054 & 0.3199 & 1.129 & 0.0370 & 1.072 \\
\hline & \multicolumn{2}{|c|}{ Zone Average $\mathrm{C} / \mathrm{E}$} & 1.070 & & 1.127 & & 0.942 \\
\hline & \multicolumn{2}{|c|}{ Standard Deviation } & 0.020 & & 0.016 & & 0.186 \\
\hline & 483.4 & 2.881 & 1.080 & 0.3696 & 1.133 & 0.0738 & 1.070 \\
\hline$O C D$ & 432.6 & 3.361 & 1.091 & 0.4244 & 1.157 & 0.0993 & 1.104 \\
\hline$O C D$ & 381.8 & 3.897 & 1.089 & 0.4935 & 1.148 & 0.1213 & 1.087 \\
\hline$O C D$ & 331.0 & 4.384 & 1.095 & 0.5559 & 1.152 & 0.1393 & 1.104 \\
\hline$O C D$ & 280.2 & 4.912 & 1.082 & 0.6138 & 1.153 & 0.1499 & 1.141 \\
\hline$O C D$ & 229.4 & 5.294 & 1.097 & 0.6732 & 1.148 & 0.1684 & 1.110 \\
\hline OC D & 178.6 & 5.759 & 1.090 & 0.7150 & 1.169 & 0.1855 & 1.089 \\
\hline OC D & 102.4 & 6.230 & 1.105 & 0.7849 & 1.167 & 0.2016 & 1.095 \\
\hline \multirow[t]{3}{*}{$O C D$} & 51.6 & 6.636 & 1.088 & 0.8245 & 1.166 & 0.2083 & 1.110 \\
\hline & \multicolumn{2}{|c|}{ Zone Average C/E } & 1.091 & & 1.155 & & 1.097 \\
\hline & Standard & iation & 0.008 & & 0.012 & & 0.024 \\
\hline
\end{tabular}




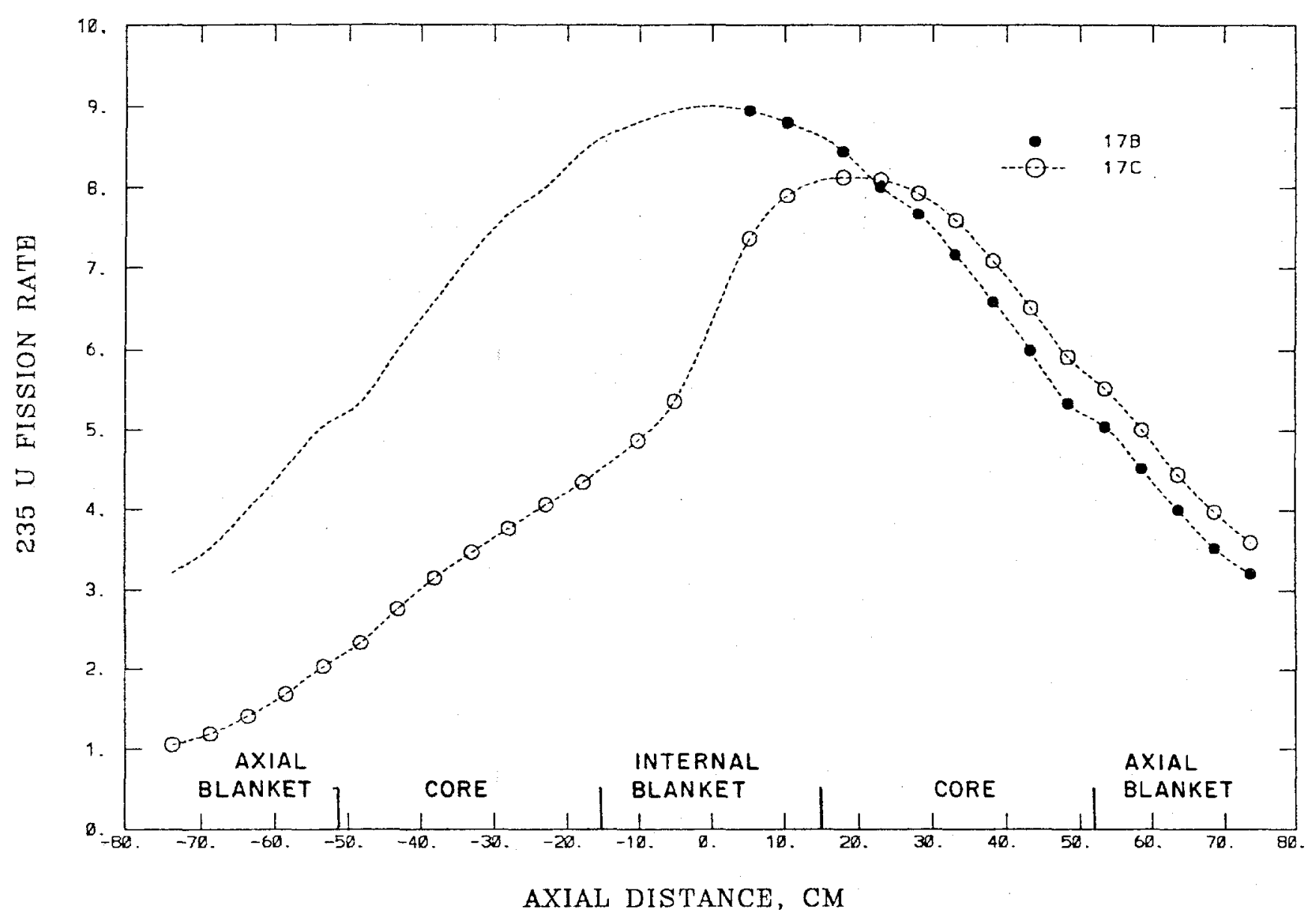

Fig. 6.8. Measured axial distributions for fission in ${ }^{235} \mathrm{U}$ adjacent to the central control position in ZPPR-17B and in ZPPR-17C. 


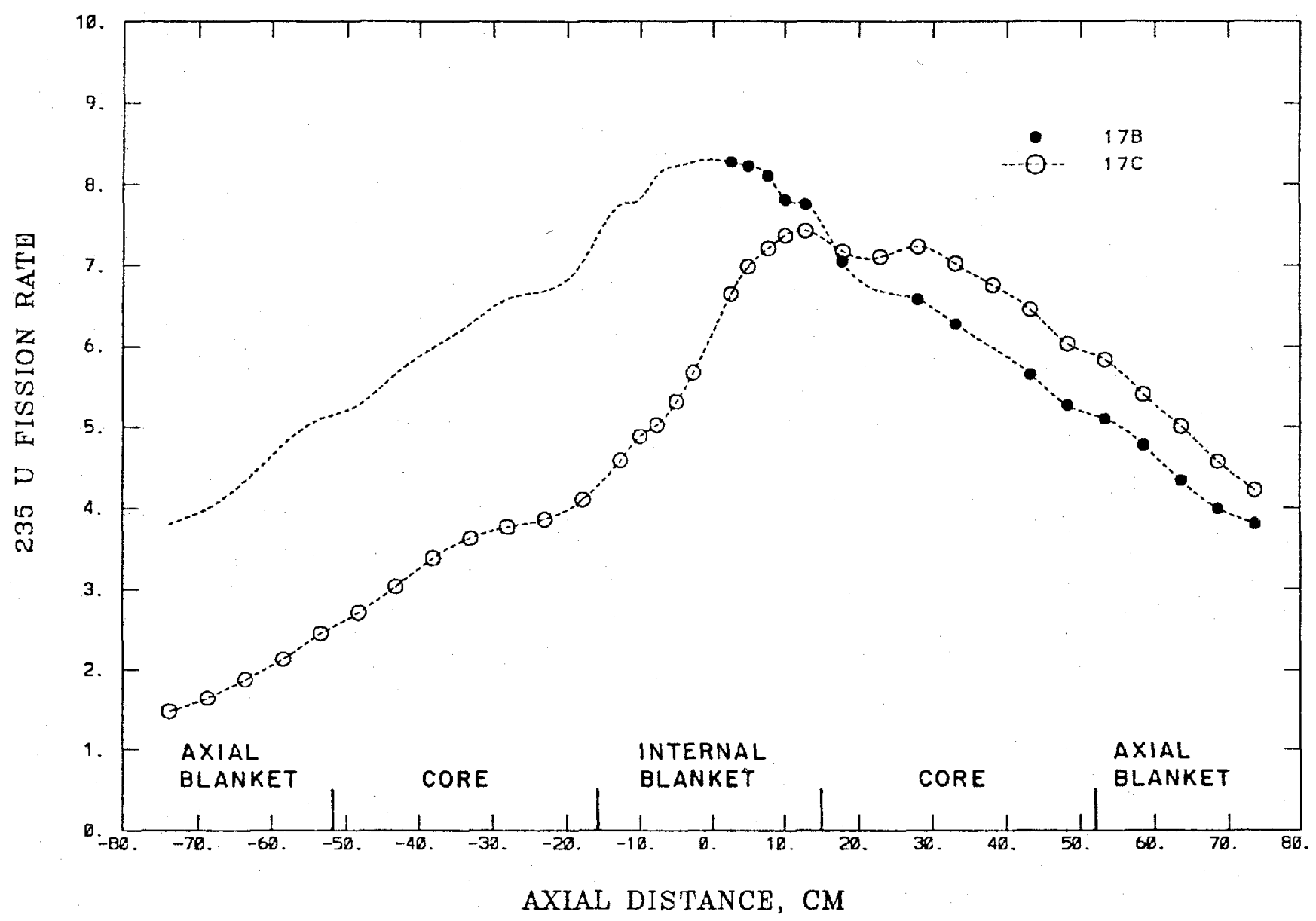

Fig. 6.9. Measured axial distributions for fission in ${ }^{235} \mathrm{U}$ adjacent to an outer control position in $2 P P R-17 B$ and in $Z P P R-17 C$. 


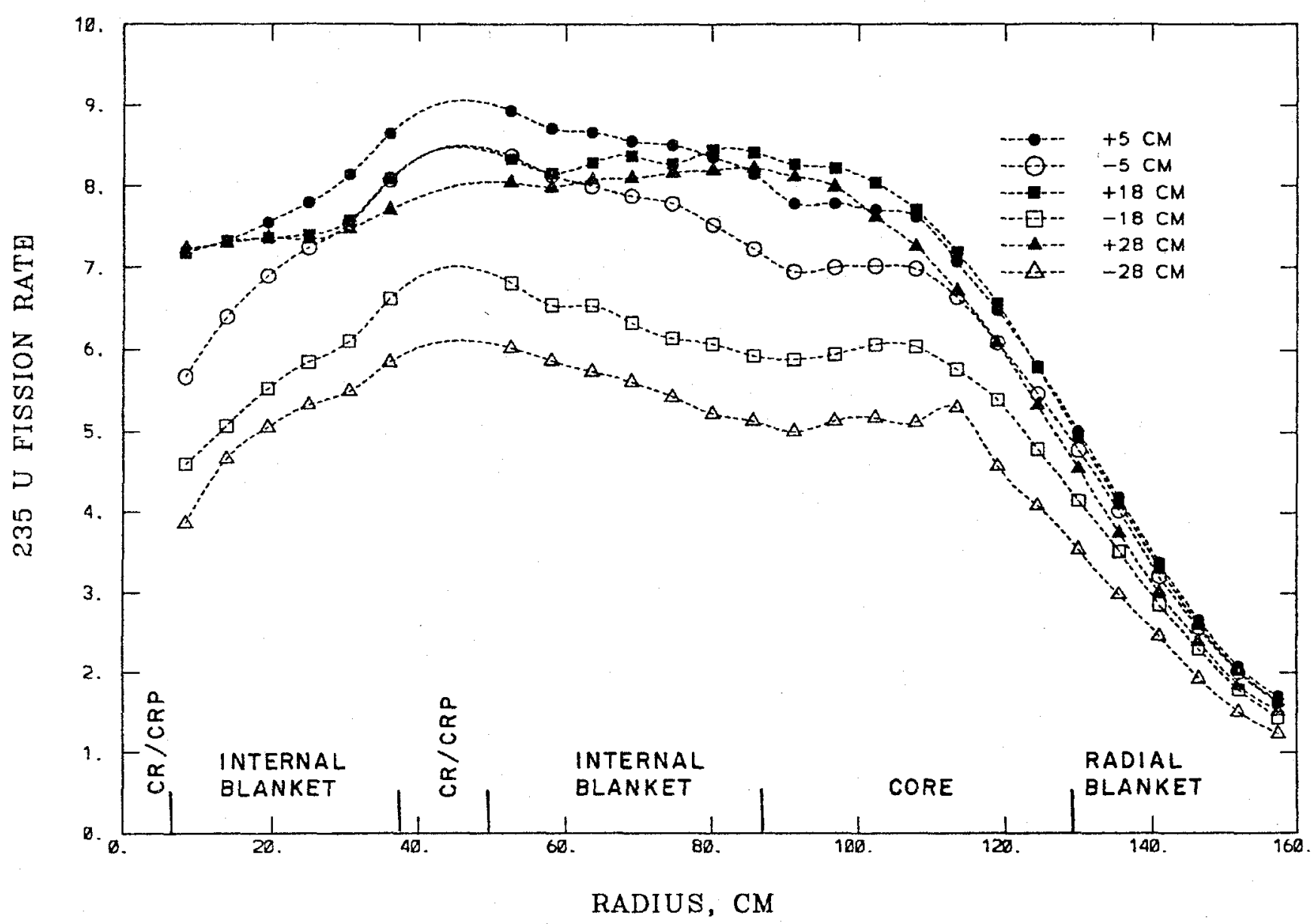

Fig. 6.10. Measured $235 \mathrm{U}$ fission distributions at six axial heights along the $x$-axis in ZPPR-17C. 


\subsection{Reaction Rate Ratios}

Experimental reaction rate ratios relative to ${ }^{239} \mathrm{Pu}$ fission were formed simply by using the cell-average values derived at each foil position. These ratios are not quite the same as those that would be obtained if all reaction rates were measured at the same xyz position. However, since the calculated values are obtained the same way, it is assumed that the effect on the $\mathrm{C} / \mathrm{E}$ ratios would be very small. The measured values quoted are accurate to within a few percent and suffice to indicate the effects of changing spectrum throughout the reactor.

The number of ratio results varies from about 40 in $17 \mathrm{~B}, 70$ in $17 \mathrm{C}$ to 90 in $17 \mathrm{~A}$. For the ${ }^{238} \mathrm{U}$ reactions more extensive data, covering a wider spectrum range, could be obtained by taking ratios relative to ${ }^{235} \mathrm{U}$ fission rather than to plutonium fission.

The detailed reaction rate ratio data on the point by point basis are given in References 3 and 5. Tables 6.17 to 6.19 show a summary of the reaction rate ratio analyses for the three phases of ZPPR-17, first giving data by traverse and by region and then giving average values for all measurements in the internal blankets and in the fuel regions.

The standard deviations of the C/E distributions for all results in the internal blanket or fuel regions are similar those of the foil statistics (about 1\% for the non-threshold reactions). This is a consequence of each reaction having a similar trend of spatial mispredictions as noted in the previous sections. Reaction rate ratio $\mathrm{C} / \mathrm{Es}$ in the radial and axial blanket regions have standard deviations significantly larger than experimental statistics due to the differing predictions as a function of distance into the blankets.

Table 6.20 compares the $C / E$ values for reaction rate ratios in the three assemblies. 
TABLE 6.17 ZPPR-17A: Summary of Reaction Rate Ratio Analysis

\begin{tabular}{|c|c|c|c|c|c|c|c|c|c|c|}
\hline \multirow[b]{2}{*}{ Distribution } & \multirow[b]{2}{*}{ Zone } & \multicolumn{3}{|c|}{$235 \mathrm{U}(\mathrm{n}, \mathrm{f}) /{ }^{239} \mathrm{Pu}(\mathrm{n}, \mathrm{f})$} & \multicolumn{3}{|c|}{${ }^{238} \mathrm{U}(\mathrm{n}, \gamma) / 2{ }^{39} \mathrm{Pu}(\mathrm{n}, \mathrm{f})$} & \multicolumn{3}{|c|}{$238 \mathrm{U}(\mathrm{n}, f) / 2{ }^{39} \mathrm{Pu}(\mathrm{n}, f)$} \\
\hline & & No. & $\mathrm{C} / \mathrm{E}$ & S.D. & No. & $\mathrm{C} / \mathrm{E}$ & S.D. & No. & $\mathrm{C} / \mathrm{E}$ & S.D. \\
\hline \multirow[t]{3}{*}{$X-A x$ is $Z=5 \mathrm{~cm}$} & IB & 16 & 1.032 & 0.007 & 16 & 1.047 & 0.013 & 16 & 1.013 & 0.043 \\
\hline & OC & 8 & 0.998 & 0.006 & 8 & 1.047 & 0.017 & 8 & 1.001 & 0.026 \\
\hline & $\mathrm{RB}$ & 5 & 1.058 & 0.043 & 5 & 1.089 & 0.025 & 5 & 0.948 & 0.118 \\
\hline \multirow[t]{3}{*}{$X$-Axis $Z=18 \mathrm{~cm}$} & IC & 11 & 1.012 & 0.012 & 11 & 1.063 & 0.007 & 11 & 0.997 & 0.019 \\
\hline & $O C$ & 8 & 0.990 & 0.007 & 8 & 1.044 & 0.016 & 8 & 1.003 & 0.030 \\
\hline & $\mathrm{RB}$ & 5 & 1.062 & 0.052 & 5 & 1.017 & 0.024 & 5 & 0.963 & 0.146 \\
\hline \multirow[t]{3}{*}{$X$-Axis $Z=28 \mathrm{~cm}$} & IC & 16 & 0.996 & 0.006 & 16 & 1.045 & 0.012 & 16 & 1.007 & 0.019 \\
\hline & OC & 8 & 0.995 & 0.006 & 8 & 1.020 & 0.020 & 8 & 0.992 & 0.028 \\
\hline & $\mathrm{RB}$ & 5 & 1.060 & 0.046 & 5 & 1.107 & 0.029 & 5 & 0.980 & 0.133 \\
\hline \multirow[t]{3}{*}{ Axial 148-50 } & IB & 5 & 1.039 & 0.014 & 5 & 1.056 & 0.009 & 5 & 0.994 & 0.057 \\
\hline & IC $\mathrm{S}$ & 7 & 1.005 & 0.011 & 7 & 1.047 & 0.017 & 7 & 1.012 & 0.024 \\
\hline & $\mathrm{AB}$ & 5 & 1.064 & 0.042 & 5 & 1.096 & 0.026 & 5 & 0.864 & 0.081 \\
\hline \multirow[t]{2}{*}{ Axial $148-66$} & $O C D$ & 9 & 1.002 & 0.010 & 9 & 1.068 & 0.010 & 9 & 1.002 & 0.024 \\
\hline & $A B$ & 5 & 1.063 & 0.040 & 5 & 1.117 & 0.027 & 5 & 0.872 & 0.109 \\
\hline \multirow[t]{2}{*}{ Axial $148-70$} & OC $\mathrm{S}$ & 9 & 1.001 & 0.011 & 9 & 1.033 & 0.012 & 9 & 1.009 & 0.020 \\
\hline & $A B$ & 5 & 1.054 & 0.048 & 5 & 1.102 & 0.043 & 5 & 0.847 & 0.130 \\
\hline 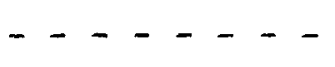 & - & _ & - & $-2-$ & _ & $-\ldots$ & - . - & 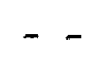 & 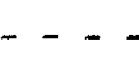 & _- \\
\hline All Internal $\mathrm{Bla}$ & inket & 21 & 1.034 & 0.009 & 20 & 1.049 & 1.012 & 20 & 1.012 & 0.044 \\
\hline All Fuel Region & & 76 & 1.000 & 0.011 & 70 & 1.050 & 0.017 & 70 & 1.004 & 0.023 \\
\hline All single Col. & Fuel & 49 & 1.001 & 0.012 & 46 & 1.043 & 0.015 & 46 & 1.008 & 0.024 \\
\hline All Double Col. & Fuel & 25 & 0.999 & 0.008 & 24 & 1.063 & 0.009 & 24 & 0.995 & 0.019 \\
\hline
\end{tabular}


TABLE 6.18 ZPPR-17B: Summary of Reaction Rate Ratio Analys is

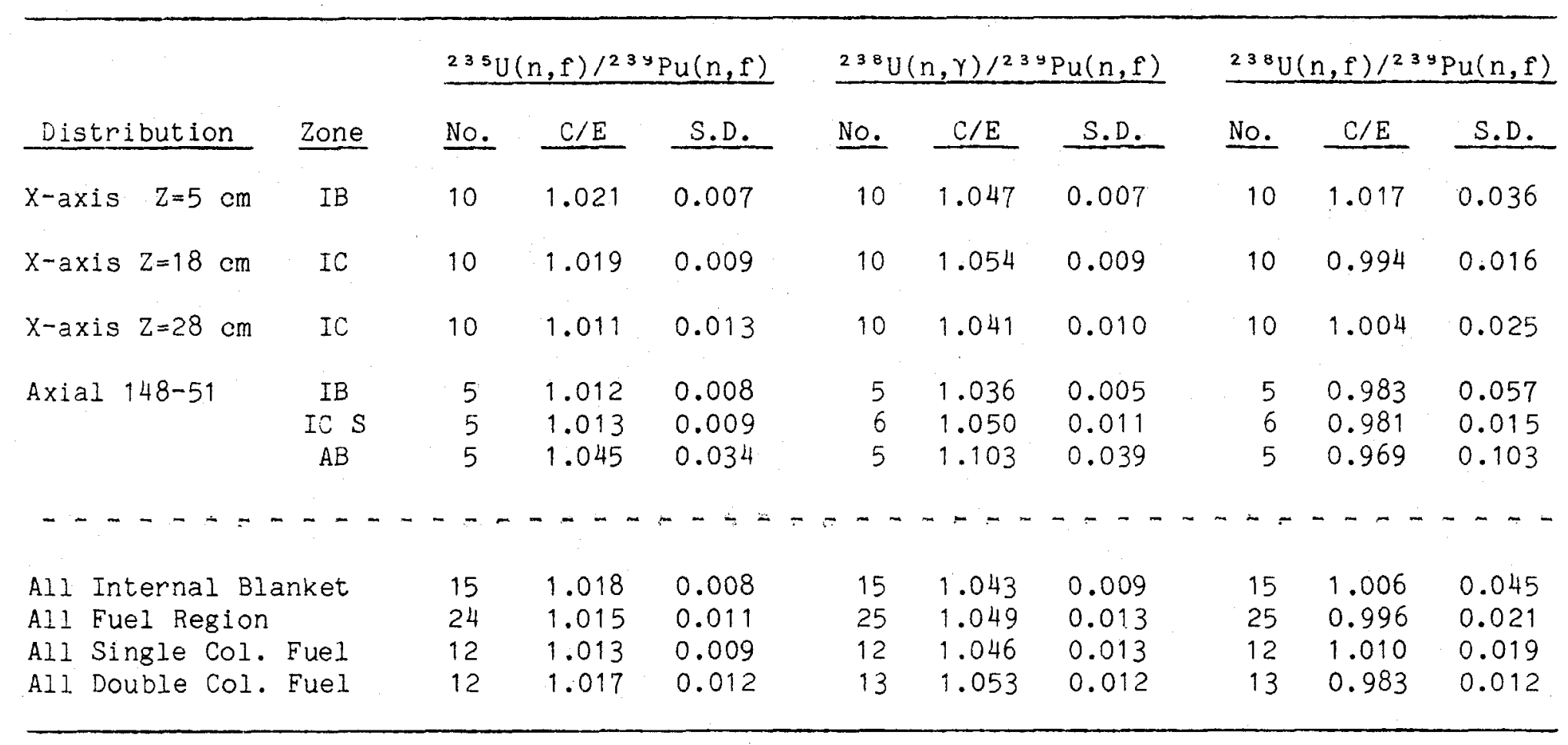


TABLE 6.19 ZPPR-17C: Summary of Reaction Rate Ratio Analysis

\begin{tabular}{|c|c|c|c|c|c|c|c|c|c|c|}
\hline \multirow[b]{2}{*}{ Distribution } & \multirow{3}{*}{$\frac{\text { Zone }}{\text { IB }}$} & \multicolumn{3}{|c|}{$235 \mathrm{U}(\mathrm{n}, \mathrm{f}) / 239 \mathrm{Pu}(\mathrm{n}, \mathrm{f})$} & \multicolumn{3}{|c|}{$23^{8} \mathrm{U}(\mathrm{n}, \mathrm{\gamma}) / 239 \mathrm{Pu}(\mathrm{n}, \mathrm{f})$} & \multicolumn{3}{|c|}{$238 \mathrm{U}(\mathrm{n}, \mathrm{f}) /{ }^{239} \mathrm{Pu}(\mathrm{n}, \mathrm{f})$} \\
\hline & & No. & $C / E$ & S.D. & No. & $\mathrm{C} / \mathrm{E}$ & S.D. & No. & $\mathrm{C} / \mathrm{E}$ & S.D. \\
\hline$X$-axis $Z=+5 \mathrm{~cm}$ & & 10 & 1.026 & 0.007 & 10 & 1.059 & 0.011 & 10 & 1.016 & 0.021 \\
\hline$X$-axis $Z=-5 \mathrm{~cm}$ & IB & 10 & 1.031 & 0.022 & 10 & 1.060 & 0.009 & 10 & 1.011 & 0.026 \\
\hline$x$-axis $z=+18 \mathrm{~cm}$ & IC & 10 & 1.012 & 0.009 & 10 & 1.069 & 0.009 & 10 & 0.994 & 0.016 \\
\hline$x$-axis $Z=-18 \mathrm{~cm}$ & IC & 10 & 1.014 & 0.020 & 10 & 1.070 & 0.014 & 10 & 1.000 & 0.025 \\
\hline$X-\operatorname{axis} Z=-28 \mathrm{~cm}$ & IC & 10 & 1.005 & 0.018 & 10 & 1.062 & 0.013 & 10 & 1.006 & 0.023 \\
\hline Axial $148-51$ & $\begin{array}{l}\text { IB } \\
\text { IC } D\end{array}$ & $\begin{array}{l}5 \\
7\end{array}$ & $\begin{array}{l}1.017 \\
1.020\end{array}$ & $\begin{array}{l}0.004 \\
0.009\end{array}$ & $\begin{array}{l}5 \\
7\end{array}$ & $\begin{array}{l}1.056 \\
1.061\end{array}$ & $\begin{array}{l}0.003 \\
0.008\end{array}$ & $\begin{array}{l}5 \\
7\end{array}$ & $\begin{array}{l}1.012 \\
0.980\end{array}$ & $\begin{array}{l}0.029 \\
0.020\end{array}$ \\
\hline Axial 248-51 & $\begin{array}{l}\text { IB } \\
\text { IC } D \\
A B \\
--~\end{array}$ & $\begin{array}{l}5 \\
7 \\
5 \\
-\end{array}$ & $\begin{array}{l}1.017 \\
1.006 \\
1.074 \\
-\ldots .\end{array}$ & $\begin{array}{l}0.009 \\
0.012 \\
0.070\end{array}$ & $\begin{array}{r}5 \\
7 \\
5 \\
-\end{array}$ & $\begin{array}{l}1.049 \\
1.047 \\
1.068 \\
-\ldots\end{array}$ & $\begin{array}{l}0.011 \\
0.009 \\
0.036\end{array}$ & $\begin{array}{r}5 \\
6 \\
5 \\
-\end{array}$ & $\begin{array}{l}1.026 \\
0.998 \\
0.989\end{array}$ & $\begin{array}{l}0.022 \\
0.012 \\
0.123\end{array}$ \\
\hline $\begin{array}{l}\text { All Internal Bla } \\
\text { All Fuel Region }\end{array}$ & set & $\begin{array}{l}30 \\
44\end{array}$ & $\begin{array}{l}1.025 \\
1.011\end{array}$ & & $\begin{array}{l}30 \\
44\end{array}$ & $\begin{array}{l}1.057 \\
1.063\end{array}$ & & $\begin{array}{l}30 \\
44\end{array}$ & $\begin{array}{l}1.015 \\
0.997\end{array}$ & \\
\hline
\end{tabular}


TABLE 6.20 Reaction Rate Ratio C/E Results for ZPPR-17A, $17 \mathrm{~B}$ and $17 \mathrm{C}$

\begin{tabular}{|c|c|c|c|c|c|}
\hline Phase & Zone & $\begin{array}{l}\text { Number } \\
\text { of Data }\end{array}$ & $\begin{array}{l}C / E \text { for } \\
\text { F25/F49 }\end{array}$ & $\begin{array}{l}\text { C/E for } \\
\text { C28/F49 } \\
\end{array}$ & $\begin{array}{l}\text { C/E for } \\
\text { F28/F49 }\end{array}$ \\
\hline $17 \mathrm{~A}$ & $\begin{array}{l}\text { Internal Blanket } \\
\text { Fuel Regions }\end{array}$ & $\begin{array}{l}20 \\
76\end{array}$ & $\begin{array}{l}1.034 \\
1.000\end{array}$ & $\begin{array}{l}1.049 \\
1.050\end{array}$ & $\begin{array}{l}1.012 \\
1.004\end{array}$ \\
\hline $17 \mathrm{~B}$ & $\begin{array}{l}\text { Internal Blanket } \\
\text { Fuel Regions }\end{array}$ & $\begin{array}{l}15 \\
24\end{array}$ & $\begin{array}{l}1.018 \\
1.015\end{array}$ & $\begin{array}{l}1.043 \\
1.049\end{array}$ & $\begin{array}{l}1.006 \\
0.996\end{array}$ \\
\hline $17 \mathrm{C}$ & $\begin{array}{l}\text { Internal Blanket } \\
\text { Fuel Regions }\end{array}$ & $\begin{array}{l}30 \\
44\end{array}$ & $\begin{array}{l}1.025 \\
1.011\end{array}$ & $\begin{array}{l}1.057 \\
1.063\end{array}$ & $\begin{array}{l}1.015 \\
0.997\end{array}$ \\
\hline
\end{tabular}




\subsection{Comparison of Fission Rate Analysis in ZPPR-17A, 17B, and 17C}

The preceding reaction rate analysis gave results which were normalised to a mean $\mathrm{C} / \mathrm{E}$ for ${ }^{23} \mathrm{Pu}$ fission in each core. Fewer plutonium foils were available in ZPPR-17B and 17C than in ZPPR-17A and were positioned around the core center. Because of the strong radial dependence of the $\mathrm{C} / \mathrm{ES}$, the results in the preceding sections must be renormalised in order to make comparisons among the three cores.

The normalisation chosen was to ${ }^{235} \mathrm{U}$ fission for common distributions in the fuel zones. These were at the $x$-axis and at $45^{\circ}$ for $z=5 \mathrm{~cm}$ (outer core only), at the $x$-axis for $z=18 \mathrm{~cm}$ and $z=28 \mathrm{~cm}$ and for axial traverses at the center, at the inner/outer core boundary, and in the outer core. Results for ZPPR-17C included both halves of the assembly. The normalising factors, relative to those used for the previous results were 1.002 for $17 \mathrm{~A}$ ( 89 foils), 1.054 for $17 \mathrm{~B}$ (96 foils), and 1.063 for 17C (192 foils).

A summary of radial distribution analyses for ${ }^{235} \mathrm{U}$ fission is given in Table 6.21. All results show a trend of increasing C/E with radius, as indicated by the ratio of the outer core average $\mathrm{C} / \mathrm{E}$ to that for the central region (ICA) shown in the last column.

The results for $\mathrm{z}=5 \mathrm{~cm}$ pass through the internal blanket and show quite a wide spread among the results, principally for the center region ICA which is near to the half-inserted control rod in ZPPR-17C. The radial discrepancies at $45^{\circ}$ are less than along the $x$-axis, possibly due to approximations in the nodal transport solution or in the interpolation procedure.

The distributions at $18 \mathrm{~cm}$ and $28 \mathrm{~cm}$ for the midplane show a monotonic increase in $\mathrm{C} / \mathrm{E}$ from the central region to the outer core. The ratios of outer core to inner (ICA) results are 1.04 (17A), 1.05 (17B), and $1.06(17 \mathrm{C})$.

The analyses of axial distributions for the three cores are given in Tables 6.22 to 6.25 . Measurements near the core center and near the edge 
of the internal blanket are adjacent to a CRP in $17 \mathrm{~B}$ and adjacent to a halfinserted control rod in $17 \mathrm{C}$. Near the core center, distributions were measured both for fission in ${ }^{239} \mathrm{Pu}$ (Table 6.22) and for fission in ${ }^{235} \mathrm{U}$ (Table 6.23). Results in the outer core, matrix 1,248-70, in Table 6.25 are about $25 \mathrm{~cm}$ removed from a control position.

The results in the fuel regions are of most interest. Fluctuations in the C/E results at different distances from the midplane are consistent with experimental statistics as shown by the standard deviations of the distributions. The mean C/E results adjacent to CRPs are systematically lower than those in the fuel regions of $17 \mathrm{~A}$ by $1 \%$ to $3 \%$. No clear trend is apparent for calculation of fission rates adjacent to a control rod compared with those adjacent to a CRP. The relative results vary by $1 \%$ at the central position for ${ }^{23} \mathrm{Pu}$ and ${ }^{235} \mathrm{U}$ fission (Tables 6.23 and 6.24) and by $2 \%$ near the outer control position (Table 6.24 ).

We conclude that the fission rates adjacent to a CRP or adjacent to a control rod can be calculated with a confidence of $2-3 \%$ relative to those in neighboring fuel regions using the nodal transport method. 
TABLE 6.21. Comparison of Radial ${ }^{235} \mathrm{U}$ Fission Rate $\mathrm{C} / \mathrm{E}$ Values in ZPPR-17A, 17B, and $17 \mathrm{C}$

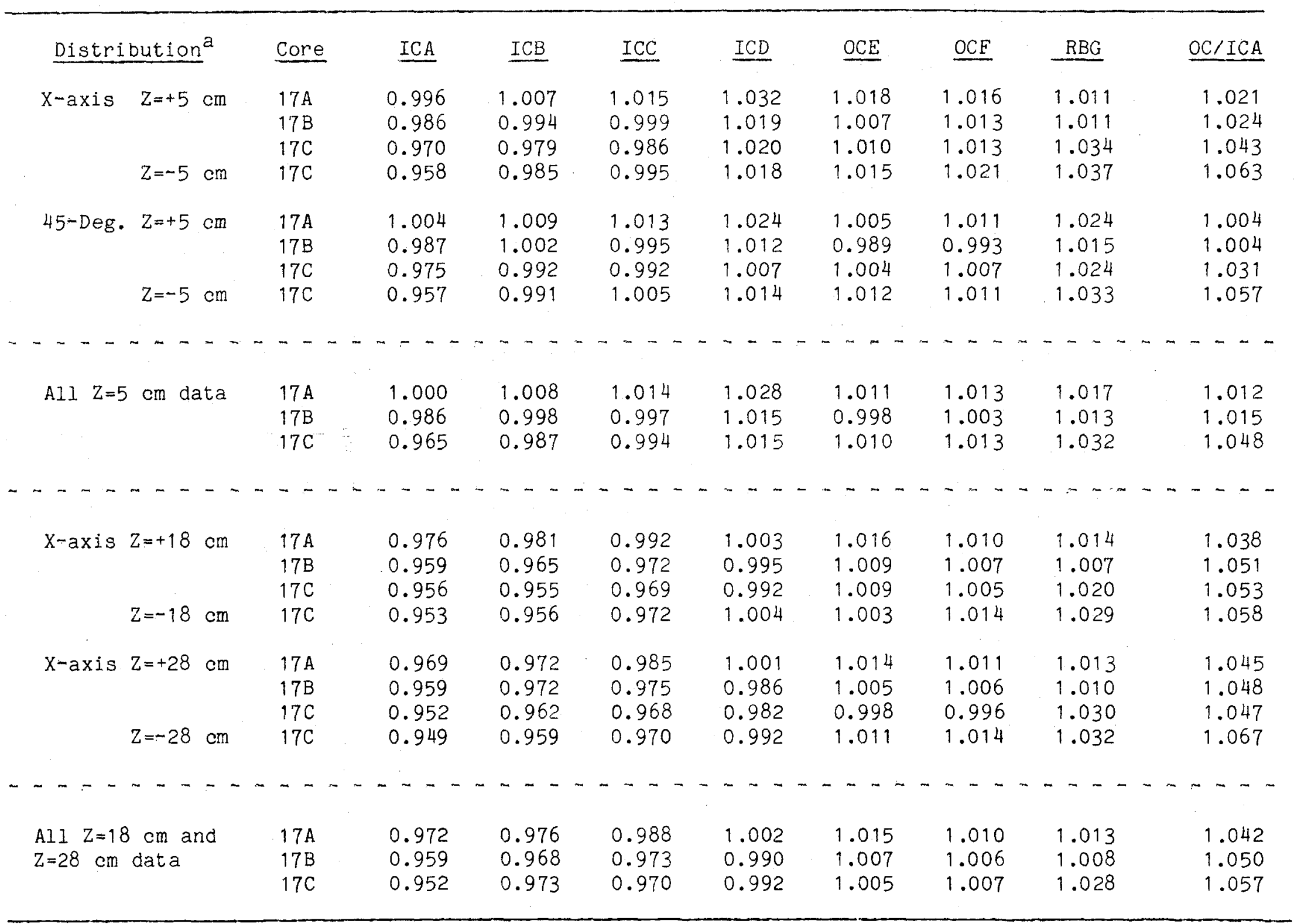

a Results shown for negative $z$ for $Z P P R-17 C$ are in half-2 which contains inserted control rods. 
TABLE 6.22. Comparison of $\mathrm{C} / \mathrm{E}$ Values for ${ }^{23 y} \mathrm{Pu}$ Fission Axial Traverses Near the Center of ZPPR-17A, 17B, and 17C

\begin{tabular}{|c|c|c|c|c|c|}
\hline \multirow[b]{2}{*}{ Zone } & \multirow[b]{2}{*}{$\underline{Z}(\mathrm{~mm})$} & \multirow{2}{*}{$\frac{\text { ZPPR }-17 \mathrm{~A}}{\text { Fuel }^{\mathrm{a}}}$} & \multirow{2}{*}{$\begin{array}{l}\text { ZPPR - 17B } \\
\text { Adj. CRP } \\
\end{array}$} & \multicolumn{2}{|c|}{ ZPPR $-17 \mathrm{C}$} \\
\hline & & & & Adj. CRP & Adj. CR \\
\hline IB & 26.2 & 0.956 & 0.966 & 0.936 & 0.933 \\
\hline IB & 49.6 & 0.945 & 0.962 & 0.945 & 0.946 \\
\hline IB & 77.0 & 0.960 & 0.962 & 0.951 & 0.929 \\
\hline IB & 100.4 & 0.964 & 0.968 & 0.942 & 0.941 \\
\hline \multirow[t]{2}{*}{ IB } & 127.8 & 0.969 & 0.965 & 0.945 & 0.943 \\
\hline & & $-m-\infty$ & $-m-\infty$ & $\cdots-\infty$ & $\cdots+\infty$ \\
\hline \multicolumn{2}{|c|}{ Zone Average $\mathrm{C} / \mathrm{E}$} & 0.959 & 0.965 & 0.944 & 0.939 \\
\hline \multicolumn{2}{|c|}{ Standard Deviation } & 0.009 & 0.003 & 0.006 & 0.007 \\
\hline IC & 178.6 & 0.963 & 0.944 & 0.949 & 0.941 \\
\hline IC & 229.4 & 0.958 & 0.952 & 0.950 & 0.944 \\
\hline IC & 280.2 & 0.974 & 0.935 & 0.942 & 0.960 \\
\hline IC & 331.0 & 0.977 & 0.950 & 0.968 & 0.964 \\
\hline IC & 381.8 & 0.987 & - & 0.950 & 0.952 \\
\hline IC & 432.6 & 0.968 & 0.941 & 0.940 & 0.948 \\
\hline \multirow[t]{2}{*}{ IC } & 483.4 & 0.958 & 0.931 & 0.929 & 0.947 \\
\hline & & $\ldots$ & $\cdots-$ & $\ldots-$ & $\ldots \ldots$ \\
\hline \multicolumn{2}{|c|}{ Zone Average $\mathrm{C} / \mathrm{E}$} & 0.969 & 0.942 & 0.947 & 0.951 \\
\hline \multicolumn{2}{|c|}{ Standard Deviation } & 0.011 & 0.009 & 0.013 & 0.009 \\
\hline$A B$ & 534.2 & 0.952 & 0.943 & - & 0.971 \\
\hline $\mathrm{AB}$ & 585.0 & 0.932 & 0.944 & - & 0.955 \\
\hline$A B$ & 635.8 & 0.920 & 0.912 & - & 0.926 \\
\hline$A B$ & 686.6 & 0.989 & 0.897 & -- & 0.907 \\
\hline \multirow[t]{2}{*}{$A B$} & 737.4 & 0.882 & 0.880 & - & 0.907 \\
\hline & & $\cdots$ & 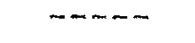 & & 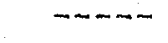 \\
\hline \multicolumn{2}{|c|}{ Zone Average $\mathrm{C} / \mathrm{E}$} & 0.917 & 0.915 & & 0.933 \\
\hline \multicolumn{2}{|c|}{ Standard Deviation } & 0.027 & 0.030 & & 0.031 \\
\hline
\end{tabular}

${ }^{a}$ In $17 \mathrm{~A}$ the measurements were made in a singlerfuel-column drawer, in $17 \mathrm{~B}$ and $17 \mathrm{C}$ they were in double-fuel-column drawers. 
TABLE 6.23. Comparison of C/E Values for ${ }^{235} \mathrm{U}$ Fission Axial Traverses Near the Center of ZPPR-17A, 17B, and 17C

\begin{tabular}{|c|c|c|c|c|c|}
\hline \multirow[b]{2}{*}{ Zone } & \multirow[b]{2}{*}{$Z(\mathrm{~mm})$} & \multirow{2}{*}{$\frac{\text { ZPPR-17A }}{\text { FueI }^{\mathrm{a}}}$} & \multirow{2}{*}{$\begin{array}{l}\text { ZPPR-17B } \\
\text { Adj. CRP }\end{array}$} & \multicolumn{2}{|c|}{$Z P P R-17 C$} \\
\hline & & & & Adj. CRP & $\mathrm{Adj} . \mathrm{CR}$ \\
\hline IB & 26.2 & 1.004 & 0.973 & 0.949 & 0.963 \\
\hline IB & 49.6 & 0.999 & 0.973 & 0.958 & 0.955 \\
\hline IB & 77.0 & 0.991 & 0.974 & 0.971 & 0.949 \\
\hline$I B$ & 100.4 & 0.992 & 0.992 & 0.961 & 0.950 \\
\hline \multirow[t]{2}{*}{ IB } & 127.8 & 0.994 & 0.969 & 0.960 & 0.955 \\
\hline & & $\ldots-$ & $\ldots \ldots$ & $\ldots$ & $\ldots$ \\
\hline \multirow{2}{*}{\multicolumn{2}{|c|}{$\begin{array}{l}\text { Zone Average C/E } \\
\text { Standard Deviation }\end{array}$}} & 0.986 & 0.976 & 0.960 & 0.955 \\
\hline & & 0.005 & 0.010 & 0.009 & 0.006 \\
\hline IC & 178.6 & 0.978 & 0.944 & 0.961 & 0.930 \\
\hline IC & 229.4 & 0.972 & $\cdots$ & 0.969 & 0.959 \\
\hline IC & 280.2 & 0.972 & 0.956 & 0.961 & 0.960 \\
\hline TC & 331.0 & 0.977 & 0.969 & 0.983 & 0.959 \\
\hline IC & 381.8 & 0.974 & - & 0.977 & 0.956 \\
\hline IC & 432.6 & 0.979 & 0.952 & 0.958 & 0.968 \\
\hline \multirow[t]{2}{*}{ IC } & 483.4 & 0.968 & 0.944 & 0.957 & 0.963 \\
\hline & & $-\infty-\infty$ & $-n-\infty$ & $\cdots \cdots$ & $-\cdots-\infty$ \\
\hline \multirow{2}{*}{\multicolumn{2}{|c|}{$\begin{array}{l}\text { Zone Average C/E } \\
\text { Standard Deviation }\end{array}$}} & 0.974 & 0.953 & 0.965 & 0.957 \\
\hline & & 0.004 & 0.010 & 0.012 & 0.013 \\
\hline$A B$ & 534.2 & 0.968 & 0.957 & 0.966 & 0.979 \\
\hline $\mathrm{AB}$ & 585.0 & 0.976 & 0.950 & 0.982 & 0.991 \\
\hline$A B$ & 635.8 & 0.963 & 0.957 & 0.983 & 0.961 \\
\hline$A B$ & 686.6 & 0.970 & 0.955 & 1.013 & 0.992 \\
\hline \multirow[t]{2}{*}{$\mathrm{AB}$} & 737.4 & 0.995 & 0.956 & 1.054 & 1.072 \\
\hline & & $\therefore-\cdots$ & 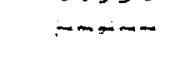 & $-\ldots-\infty$ & $\cdots \cdots$ \\
\hline \multirow{2}{*}{\multicolumn{2}{|c|}{$\begin{array}{l}\text { Zone Average C/E } \\
\text { Standard Deviation }\end{array}$}} & 0.974 & 0.955 & 1.000 & 1.000 \\
\hline & & 0.012 & 0.003 & 0.037 & 0.045 \\
\hline
\end{tabular}

${ }^{a}$ In $17 \mathrm{~A}$ the measurements were made in a single-fuel-column drawer, in $17 \mathrm{~B}$ and $17 \mathrm{C}$ they were in double-fuel-column drawers. 
TABLE 6.24. Comparison of C/E Values for ${ }^{235} \mathrm{U}$ Fission Axial Traverses Near the Edge of the Inner Blanket in ZPPR-17A, 17B, and 17C

\begin{tabular}{|c|c|c|c|c|c|}
\hline \multirow[b]{2}{*}{ Zone $^{\mathrm{a}}$} & \multirow[b]{2}{*}{$\underline{Z(\mathrm{~mm})}$} & \multirow{2}{*}{$\frac{\text { ZPPR - 17A }}{\underline{\text { Fuel }}}$} & \multirow{2}{*}{$\begin{array}{l}\text { ZPPR-17B } \\
\text { Adj. CRP }\end{array}$} & \multicolumn{2}{|c|}{ ZPPR-17C } \\
\hline & & & & Adj. CRP & Adj. CR \\
\hline OC & 51.6 & 1.020 & 0.987 & 0.983 & 1.009 \\
\hline$O C$ & 102.4 & 1.029 & 0.988 & 0.989 & 0.995 \\
\hline OC & 178.6 & 1.017 & 0.985 & 0.992 & 1.010 \\
\hline OC & 229.4 & 1.012 & 0.993 & 0.982 & 1.012 \\
\hline OC & 280.2 & 1.007 & 0.984 & 0.980 & 1.016 \\
\hline OC & 331.0 & 1.001 & 0.984 & 0.990 & 1.014 \\
\hline OC & 381.8 & 1.007 & 0.987 & 0.987 & 1.000 \\
\hline $\mathrm{OC}$ & 432.6 & 1.005 & 0.984 & 0.977 & 0.992 \\
\hline \multirow[t]{3}{*}{$\mathrm{OC}$} & 483.4 & 1.005 & 0.987 & 0.973 & 0.994 \\
\hline & & 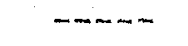 & $m-m-$ & $-\cdots$, & $\cdots-m$ \\
\hline & Zone Average & 1.011 & 0.987 & 0.984 & 1.005 \\
\hline \multicolumn{2}{|c|}{ Standard Deviation } & 0.009 & 0.003 & 0.007 & 0.010 \\
\hline$A B$ & 534.2 & 1.013 & 0.985 & 0.979 & 0.976 \\
\hline$A B$ & 585.0 & 1.000 & 0.988 & 0.988 & 0.992 \\
\hline$A B$ & 635.8 & 1.006 & 0.989 & 1.002 & 0.978 \\
\hline$A B$ & 686.6 & 1.009 & 1.007 & 1.027 & 0.996 \\
\hline \multirow[t]{3}{*}{$A B$} & 737.4 & 1.042 & 1.029 & 1.063 & 1.024 \\
\hline & & $m-\infty$ & $\cdots-\infty$ & $\cdots-$ & $\cdots$ \\
\hline & Zone Average & 1.014 & 0.999 & 1.012 & 0.993 \\
\hline \multicolumn{2}{|c|}{ Standard Deviation } & 0.016 & 0.020 & 0.036 & 0.020 \\
\hline
\end{tabular}

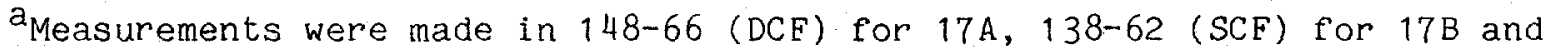
in $138 / 238-62$ (DCF) for $17 \mathrm{C}$. 
TABLE 6.25. Comparison of C/E Values for ${ }^{235} \mathrm{U}$ Fission Axial Traverses in 1,248-70 (Outer Core) in ZPPR -17A, $17 \mathrm{~B}$ and $17 \mathrm{C}$

\begin{tabular}{|c|c|c|c|c|c|}
\hline \multirow[b]{2}{*}{ Zone } & \multirow[b]{2}{*}{$\underline{Z(\mathrm{~mm})}$} & \multirow{2}{*}{$\frac{\text { ZPPR-17A }}{\text { Fue1 }}$} & \multirow{2}{*}{$\frac{\text { ZPPR-17B }}{\text { Fuel }}$} & \multicolumn{2}{|c|}{ ZPPR $-17 \mathrm{C}$} \\
\hline & & & & Half -1 & Half -2 \\
\hline OC & 51.6 & 1.022 & 1.008 & 1.024 & 1.024 \\
\hline$O C$ & 102.4 & 1.018 & 1.042 & 1.025 & 1.040 \\
\hline $\mathrm{OC}$ & 178.6 & 1.017 & 1.011 & 1.011 & 1.025 \\
\hline OC & 229.4 & 1.014 & 1.005 & 1.010 & 1.032 \\
\hline OC & 280.2 & 1.010 & 1.007 & 1.005 & 1.018 \\
\hline$O C$ & 331.0 & 1.016 & 1.008 & 1.026 & 1.030 \\
\hline OC & 381.8 & 1.019 & 1.011 & 1.024 & 1.024 \\
\hline$O C$ & 432.6 & 1.018 & 0.998 & 1.018 & 1.026 \\
\hline \multirow[t]{2}{*}{ OC } & 483.4 & 1.003 & 0.998 & 1.013 & 1.016 \\
\hline & & $\rightarrow \rightarrow-\infty$ & 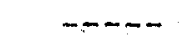 & 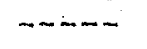 & $-\cdots-$ \\
\hline \multirow{2}{*}{\multicolumn{2}{|c|}{$\begin{array}{l}\text { Zone Average } \\
\text { Standard Deviation }\end{array}$}} & 1.015 & 1.009 & 1.017 & 1.026 \\
\hline & & 0.006 & 0.014 & 0.008 & 0.008 \\
\hline$A B$ & 534.2 & 1.000 & 0.986 & 0.984 & 0.992 \\
\hline$A B$ & 585.0 & 0.999 & 1.007 & 0.986 & 1.005 \\
\hline$A B$ & 635.8 & 0.995 & 0.996 & 0.990 & 0.988 \\
\hline$A B$ & 686.6 & 1.013 & 0.996 & 1.021 & 1.013 \\
\hline \multirow[t]{3}{*}{$A B$} & 737.4 & 1.040 & 1.028 & 1.043 & 1.035 \\
\hline & & $m \rightarrow+m$ & $m-m$ & $m-m-\infty$ & $-\infty \cdots-$ \\
\hline & Zone Average & 1.009 & 1.003 & 1.005 & 1.007 \\
\hline \multicolumn{2}{|c|}{ Standard Deviation } & 0.018 & 0.017 & 0.028 & 0.020 \\
\hline
\end{tabular}

${ }^{2}$ Single-fuel-column drawer (SCF) in $17 \mathrm{~A}$ and $17 \mathrm{~B}$, double-fuel-column drawer (DCF) in $17 \mathrm{C}$. 


\title{
7. ANALYSIS OF GAMMA HEATING MEASUREMENTS IN ZPPR-17A
}

Gamma dose measurements using thermoluminescent dosimeters (TLDs) were made in the three phases of ZPPR-17.5 Analysis has been completed only for ZPPR-17A. This work was done by $\mathrm{H}$. Unesaki and is reported in detail in reference 10 . This section summarizes the results of the analysis.

The analysis used the system recently developed by the Applied Physics Division in ANL-E for use in LMR design. The starting point is a base production library for $\mathrm{MC}^{2}$ with 230 neutron and 94 gamma groups which was generated from the ENDF/B-5.2 data using the NJOY code. The calculation procedure for ZPPR-17 involved several steps as follows:

(i) Cell-averaging and group collapse using the SDX code, producing

\author{
21 group neutron library \\ 21 group gamma interaction library \\ 21-neutron/21-gamma group coupled library (PMATRX).
}

For calculations of heating distributions within the ZPPR cells, in-plate cross sections for the $\mathrm{Pu}$ and $\mathrm{U}$ isotopes were generated in addition to cell-average data.

(ii) A 21 group neutron flux calculation using the nodal transport method with an xyz model. Calculation of a gamma source file by mesh and group using the GAMSOR-2 code with the PMATRX library.

(iii) A 21 group gamma flux calculation in the xyz nodal transport model using the gamma source file as input and the gamma interaction library.

(iv) Edit of the gamma dose in steel using a code written at ZPPR called TOTDOSE. The inputs to the code are neutron and gamma flux files, neutron and gamma response functions for the TLDs and mass-energy absorption coefficients for steel. 
The measured dose distributions give values in a sodium plate within each drawer. For comparison with calculated cell-average doses, factors relating dose rates in the position of the measurement to the cell average are calculated by

(v) Calculations for the cell using the TWODANT code following steps (ii) to (iv) above. TLD/cell average factors are calculated with the TOTDOSE code. The cell calculations were made with $S_{16} P_{0}$ (transport corrected) for neutron fluxes and $S_{16} P_{3}$ for the gamma fluxes, using the double $\mathrm{P}_{\mathrm{N}}$ approximation. Three mesh intervals in diluent plates and five mesh intervals in $\mathrm{Pu}$ and $\mathrm{U}$ regions were used.

The positions of the gamma dose measurements in ZPPR-17A are shown in Fig. 7.1. Several in-cell measurements were also made to test calculated distributions. In these, the TLDs were placed in small indentations in sodium-plate clads or in special uranium plates. Results for a double-fuelcolumn cell are shown in Fig. 7.2. Measured distributions in the singlefuel-column cell were rather uninteresting since it was not possible to position a TLD near the fuel plate. ${ }^{\circ}$ Several multiple-cell calculations were made, since the cell factors for a given cell varied with its environment. The results are shown in Table 7.1. The most appropriate factors were used for each cell occurring in the reactor distributions.

The quantities calculated in the TOTDOSE code are as follows:

$$
\begin{aligned}
\text { Neutron }= & D_{n}={ }_{E}{ }_{n}(E) \cdot S(E), \\
\text { gamma }= & D_{Y}={ }_{E}{ }^{\phi_{\gamma}(E) \cdot\left(\frac{\mu}{\rho}\right)}{ }_{S S}(E) \cdot f(E) \cdot \bar{E}(E), \\
\text { where }= & \phi_{n}(E): \quad \text { neutron flux for group } E \\
& S(E): \quad \text { neutron sensitivity of } T L D \text { for group E } \\
& \phi_{\gamma}(E): \quad \text { Gamma Flux for group } E \\
& \left(\frac{\mu}{\rho}\right)_{S S}(E): \text { Mass energy absorption coefficient of } \\
& \text { stainless steel for group } E
\end{aligned}
$$




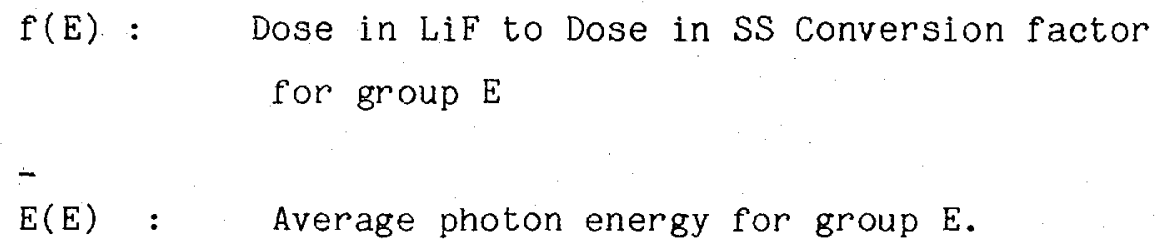

The energy structures of the neutron and gamma libraries are given in Table 7.2. Sensitivity factors are given in Table 7.3 and mass energy absorption coefficients for steel are in Table 7.4 .

The calculated and measured dose rates are compared on an absolute basis by normalizing to ${ }^{23 y} \mathrm{Pu}$ fission rates. The measurements were normalized to fission rates from a foil irradiation using the ex-core monitors. Calculations were normalized using the mean C/E obtained from 62 measurements of fission in ${ }^{23 y} \mathrm{Pu}$ in $\mathrm{ZPPR}-17 \mathrm{~A}$.

Calculated and experimental dose rates are compared for x-axis distributions in Tables 7.5 and 7.6 and for three axial distributions in Tables 7.7 to 7.9 . The results are also plotted in Figs. 7.3 and 7.4 .

The mean $\mathrm{C} / \mathrm{E}$ for dose in the fuel regions relative to ${ }^{23 y} \mathrm{Pu}$ fission is 0.95. However, a systematic difference is obtained between results in the single-fuel-column cells (mean $C / E=0.99$ ) and the double-column fuel cells (mean $C / E=0.90)$. This difference may be due to inadequacies in calculations of the variations of gamma dose through the unit cells. The problem is similar to that found for ${ }^{238} \mathrm{U}$ fission rates and may have a common source. The dose distributions in the core are mispredicted by $5 \%$ as a function of radius, as would be expected from the analysis of fission rates. The results for the internal blanket are very good with a mean $C / E=1.00$. Only six results are available in the axial blanket but these are well calculated (mean $\mathrm{C} / \mathrm{E}=0.98$ ). Five results in the radial blanket have a mean $\mathrm{C} / \mathrm{E}$ of 0.90 . 
TABLE 7.1 Summary of Cell Averaging Factors

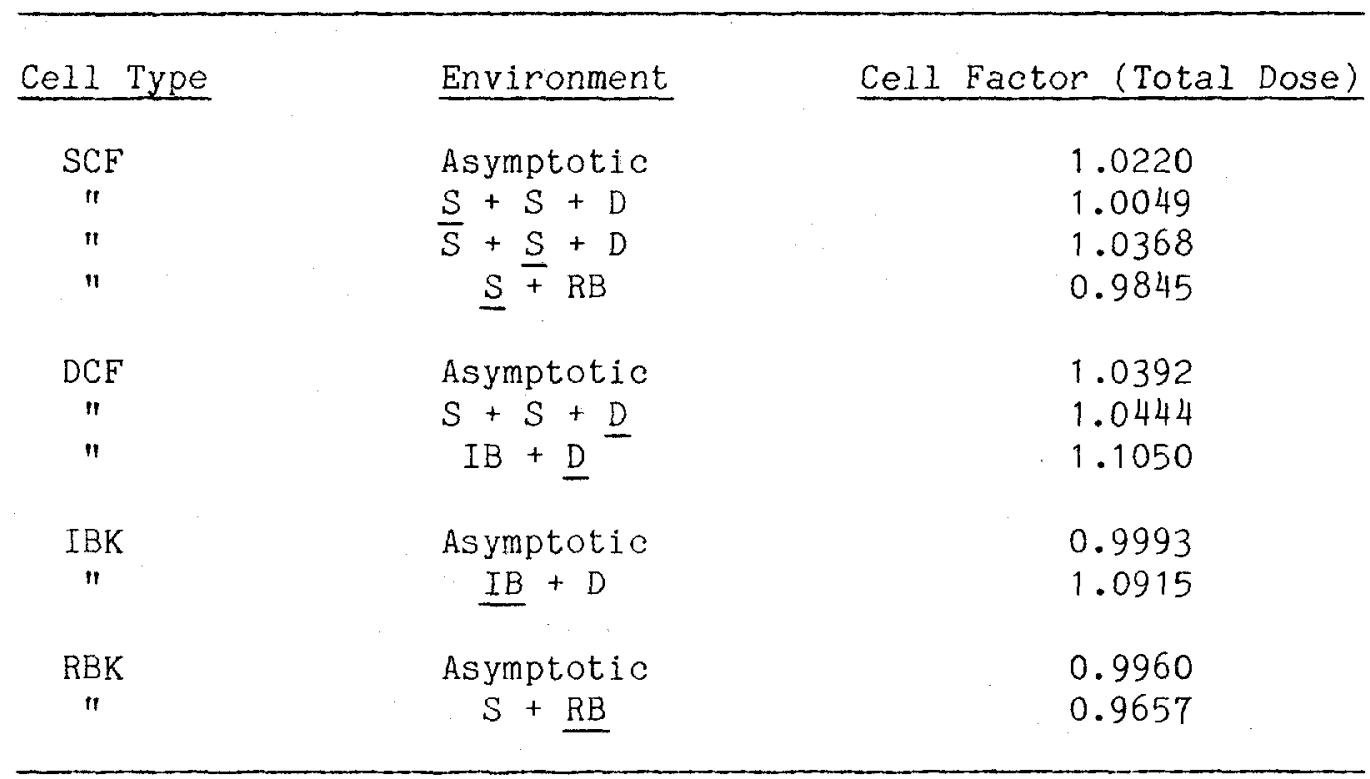


TABLE 7.2 Broad Group Structures for Neutron and Gamma Cross Section Library

\begin{tabular}{c} 
Broad Group \\
Number \\
\hline 1 \\
2 \\
3 \\
4 \\
5 \\
6 \\
7 \\
8 \\
9 \\
10 \\
11 \\
12 \\
13 \\
14 \\
15 \\
16 \\
17 \\
18 \\
19 \\
20 \\
21
\end{tabular}

\begin{tabular}{ll}
\multicolumn{2}{c}{ Upper Bound, eV } \\
\hline Neutron & Gamma \\
\hline $1.41907+7$ & $2.00000+7$ \\
$6.06531+6$ & $1.00000+7$ \\
$3.67879+6$ & $8.00000+6$ \\
$2.23130+6$ & $7.00000+6$ \\
$1.35335+6$ & $6.00000+6$ \\
$8.20850+5$ & $5.00000+6$ \\
$4.97871+5$ & $4.00000+6$ \\
$3.01974+5$ & $3.00000+6$ \\
$1.83156+5$ & $2.50000+6$ \\
$1.11090+5$ & $2.00000+6$ \\
$6.73795+4$ & $1.50000+6$ \\
$4.08677+4$ & $1.00000+6$ \\
$2.47875+4$ & $7.00000+5$ \\
$1.50344+4$ & $4.50000+5$ \\
$9.11883+3$ & $3.00000+5$ \\
$5.53085+3$ & $1.50000+5$ \\
$3.35463+3$ & $1.00000+5$ \\
$2.03469+3$ & $2.50000+4$ \\
$1.23410+3$ & $4.50000+4$ \\
$4.54000+2$ & $3.00000+4$ \\
$6.14423+1$ & $2.00000+4$
\end{tabular}


TABLE 7.3 Neutron and Gamma Response Functions for TLDS

\begin{tabular}{rlc}
\hline Group & Neutron $S(E)$ & Gamma $f(E)$ \\
\hline 1 & $3.20027 \mathrm{E}-10$ & 1.06001 \\
2 & $1.85294 \mathrm{E}-10$ & 1.05745 \\
3 & $1.05852 \mathrm{E}-10$ & 1.05828 \\
4 & $6.19877 \mathrm{E}-11$ & 1.06023 \\
5 & $3.91033 \mathrm{E}-11$ & 1.06297 \\
6 & $2.35668 \mathrm{E}-11$ & 1.06685 \\
7 & $2.36631 \mathrm{E}-11$ & 1.07483 \\
8 & $1.84305 \mathrm{E}-11$ & 1.08027 \\
9 & $5.73654 \mathrm{E}-12$ & 1.08257 \\
10 & $3.28982 \mathrm{E}-12$ & 1.07968 \\
11 & $4.73238 \mathrm{E}-12$ & 1.06549 \\
12 & $2.80550 \mathrm{E}-12$ & 1.04134 \\
13 & $9.61333 \mathrm{E}-13$ & 1.00073 \\
14 & $5.93583 \mathrm{E}-13$ & 0.91057 \\
15 & $4.35426 \mathrm{E}-13$ & 0.62258 \\
16 & $3.16693 \mathrm{E}-13$ & 0.21927 \\
17 & $2.10752 \mathrm{E}-13$ & 0.08248 \\
18 & $1.44300 \mathrm{E}-13$ & 0.04020 \\
19 & $7.29792 \mathrm{E}-14$ & 0.02764 \\
20 & $2.87526 \mathrm{E}-14$ & 0.02843 \\
21 & $4.54593 \mathrm{E}-14$ & 0.03775 \\
\hline
\end{tabular}


TABLE 7.4 Mass Absorption Coefficients for Stainless Steel

\begin{tabular}{ccc}
\hline Gamma Group & $E(\mathrm{eV})$ & $\frac{\left(\frac{\mu}{p}\right) \mathrm{a}}{\mathrm{p} S}$ \\
\cline { 2 - 3 } 1 & $1.5+7$ & 0.0224 \\
2 & $9.0+6$ & 0.0210 \\
3 & $7.5+6$ & 0.0206 \\
4 & $6.5+6$ & 0.0203 \\
5 & $5.5+6$ & 0.0200 \\
6 & $4.5+6$ & 0.0199 \\
7 & $3.5+6$ & 0.0202 \\
8 & $2.75+6$ & 0.0209 \\
9 & $2.25+6$ & 0.0217 \\
10 & $1.75+6$ & 0.0230 \\
11 & $1.25+6$ & 0.0250 \\
12 & $8.5+5$ & 0.0269 \\
13 & $5.75+5$ & 0.0287 \\
14 & $3.75+5$ & 0.0311 \\
15 & $2.25+5$ & 0.0440 \\
16 & $1.25+5$ & 0.1458 \\
17 & $8.75+4$ & 0.3320 \\
18 & $6.0+4$ & 0.9382 \\
19 & $3.75+4$ & 4.1026 \\
20 & $2.50+4$ & 14.7236 \\
21 & $1.25+4$ & 92.2460 \\
\hline
\end{tabular}

${ }^{a}$ Stainless steel composition is $18 \% \mathrm{Cr}, 2 \% \mathrm{Mn}$, $70 \% \mathrm{Fe}$ and $10 \% \mathrm{Ni}$. 
TABLE 7.5 Measured and Calculated Dose Rates along the $\mathrm{X}$-axis at 3 in. from the Midplane

\begin{tabular}{|c|c|c|c|c|c|c|}
\hline Location & $\begin{array}{r}\text { Drawer } \\
\text { Type } \\
\end{array}$ & Exp. & $\begin{array}{c}\text { Cell } \\
\text { Factor } \\
\end{array}$ & $\begin{array}{c}\text { Corrected } \\
\text { Exp. }\end{array}$ & $\mathrm{Calc}^{\mathrm{c}}$ & $\mathrm{C} / \mathrm{E}$ \\
\hline $149-50$ & SF & 0.1601 & 0.9993 & 0.1600 & $0.85903 E-03$ & 0.9514 \\
\hline $149-51$ & $\mathrm{DF}$ & 0.1625 & 0.9993 & 0.1624 & $0.86263 \mathrm{E}-03$ & 0.9412 \\
\hline $149-52$ & SF & 0.1612 & 0.9993 & 0.1611 & $0.86386 E-03$ & 0.9502 \\
\hline $149-53$ & $\mathrm{SF}$ & 0.1600 & 0.9993 & 0.1599 & $0.87177 \mathrm{E}-03$ & 0.9661 \\
\hline $149-54$ & $\mathrm{DF}$ & 0.1622 & 0.9993 & 0.1621 & $0.88390 \mathrm{E}-03$ & 0.9662 \\
\hline $149-55$ & SF & 0.1636 & 0.9993 & 0.1635 & $0.89344 E-03$ & 0.9683 \\
\hline $149-56$ & SF & 0.1655 & 0.9993 & 0.1654 & $0.91013 \mathrm{E}-03$ & 0.9751 \\
\hline $149-57$ & $D F$ & 0.1663 & 0.9993 & 0.1662 & $0.93169 \mathrm{E}-03$ & 0.9934 \\
\hline $149-58$ & $\mathrm{SF}$ & 0.1667 & 0.9993 & 0.1666 & $0.95112 E-03$ & 1.0117 \\
\hline $149-59$ & $\mathrm{SF}$ & 0.1789 & 0.9993 & 0.1788 & $0.97890 \mathrm{E}-03$ & 0.9702 \\
\hline $149-60$ & $\mathrm{DF}$ & 0.1781 & 0.9993 & 0.1780 & $0.10129 \mathrm{E}-02$ & 1.0084 \\
\hline $149-61$ & $\mathrm{SF}$ & 0.1866 & 0.9993 & 0.1865 & $0.10462 \mathrm{E}-02$ & 0.9941 \\
\hline $149-62$ & $\mathrm{SF}$ & 0.1896 & 0.9993 & 0.1895 & $0.10920 \mathrm{E}-02$ & 1.0212 \\
\hline $149-63$ & $\mathrm{DF}$ & 0.2002 & 0.9993 & 0.2001 & $0.11538 \mathrm{E}-02$ & 1.0219 \\
\hline $149-64$ & $\mathrm{SF}$ & 0.2136 & 0.9993 & 0.2135 & $0.12480 \mathrm{E}-02$ & 1.0360 \\
\hline $149-65$ & SF & 0.2489 & 1.0915 & 0.2717 & $0.15602 \mathrm{E}-02$ & 1.0176 \\
\hline $149-66$ & $D F$ & 0.4885 & 1.1050 & 0.5398 & $0.27325 E-02$ & 0.8969 \\
\hline $149-67$ & $\mathrm{SF}$ & 0.4698 & 1.0049 & 0.4721 & $0.26702 \mathrm{E}-02$ & 1.0021 \\
\hline $149-68$ & $\mathrm{SF}$ & 0.4434 & 1.0368 & 0.4597 & $0.26691 \mathrm{E}-02$ & 1.0287 \\
\hline $149-69$ & DF & 0.5065 & 1.0444 & 0.5290 & $0.27598 \mathrm{E}-02$ & 0.9244 \\
\hline $149-70$ & $\mathrm{SF}$ & 0.4204 & 1.0049 & 0.4225 & $0.23253 E-02$ & 0.9752 \\
\hline $149-71$ & SF & 0.3573 & 1.0368 & 0.3704 & $0.21000 \mathrm{E}-02$ & 1.0044 \\
\hline $149-72$ & $\mathrm{DF}$ & 0.3642 & 1.0444 & 0.3804 & $0.19379 E-02$ & 0.9027 \\
\hline $149-73$ & SF & 0.2510 & 0.9845 & 0.2471 & $0.13487 \mathrm{E}-02$ & 0.9671 \\
\hline $149-74$ & B & 0.1330 & 0.9657 & 0.1284 & $0.64630 \mathrm{E}-03$ & 0.8916 \\
\hline $149-75$ & B & 0.0814 & 0.9960 & 0.0811 & $0.39647 \mathrm{E}-03$ & 0.8665 \\
\hline $149-76$ & $\mathrm{~B}$ & 0.0555 & 0.9960 & 0.0553 & $0.27384 \mathrm{E}-03$ & 0.8778 \\
\hline $149-77$ & B & 0.0377 & 0.9960 & 0.0375 & $0.19310 \mathrm{E}-03$ & 0.9112 \\
\hline $149-78$ & B & 0.0258 & 0.9960 & 0.0257 & $0.13518 E-03$ & 0.9321 \\
\hline $149-79$ & $\mathrm{R}$ & 0.0171 & 1.0000 & 0.0171 & $0.71451 \mathrm{E}-04$ & 0.7404 \\
\hline $149-80$ & $\mathrm{R}$ & 0.0127 & 1.0000 & 0.0127 & $0.39789 E-04$ & 0.5551 \\
\hline $149-81$ & $\mathrm{R}$ & 0.0076 & 1.0000 & 0.0076 & $0.17826 \mathrm{E}-04$ & 0.4156 \\
\hline
\end{tabular}

${ }^{a}$ SF is single fuel column DF is double fuel column, $B$ is blanket and $R$ is reflector.

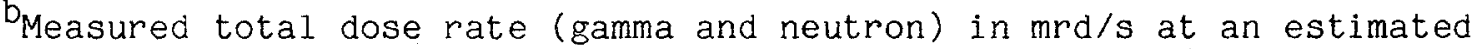
reactor power of 1 watt. Statistical uncertainties $2 \%$.

${ }^{c}$ Nodal transport calculation in xyz geometry with fluxes normalized to 8 watts, calculated doses in rads. For comparison with measurement the calculated doses are normalized by the mean $\mathrm{C} / \mathrm{E}$ for ${ }^{2}{ }^{39} \mathrm{Pu}$ fission from 62 foil measurements in the core region. 
TABLE 7.6 Measured and Calculated Dose Rates along the $\mathrm{X}$-Axis at 9 in. from the Midplane

\begin{tabular}{|c|c|c|c|c|c|c|}
\hline Location & $\begin{array}{l}\text { Drawer } \\
\text { Type a } \\
\end{array}$ & Exp.b & $\begin{array}{c}\text { Cell } \\
\text { Factor } \\
\end{array}$ & $\begin{array}{c}\text { Corrected } \\
\text { Exp. } \\
\end{array}$ & Calc. ${ }^{\mathrm{C}}$ & $C / E$ \\
\hline $149-50$ & SF & 0.3573 & 1.0368 & 0.3704 & $0.19986 \mathrm{E}-02$ & 0.9559 \\
\hline $149-51$ & DF & 0.4248 & 1.0444 & 0.4437 & $0.21643 \mathrm{E}-02$ & 0.8644 \\
\hline $149-52$ & SF & 0.3640 & 1.0049 & 0.3658 & $0.19721 \mathrm{E}-02$ & 0.9553 \\
\hline $149-53$ & $\mathrm{SF}$ & 0.3523 & 1.0368 & 0.3653 & $0.20115 \mathrm{E}-02$ & 0.9758 \\
\hline $149-54$ & DF & 0.4359 & 1.0444 & 0.4553 & $0.22078 E-02$ & 0.8593 \\
\hline $149-55$ & SF & 0.3776 & 1.0049 & 0.3795 & $0.20282 \mathrm{E}-02$ & 0.9471 \\
\hline $149-56$ & SF & 0.3607 & 1.0368 & 0.3740 & $0.20845 E-02$ & 0.9876 \\
\hline $149-57$ & DF & 0.4477 & 1.0444 & 0.4676 & $0.23047 \mathrm{E}-02$ & 0.8733 \\
\hline $149-58$ & $\mathrm{SF}$ & 0.3897 & 1.0049 & 0.3916 & $0.21311 \mathrm{E}-02$ & 0.9642 \\
\hline $149-59$ & SF & 0.3786 & 1.0368 & 0.3925 & $0.22033 \mathrm{E}-02$ & 0.9945 \\
\hline $149-60$ & DF & 0.4592 & 1.0444 & 0.4796 & $0.24484 E-02$ & 0.9046 \\
\hline $149-61$ & SF & 0.4176 & 1.0049 & 0.4196 & $0.22709 \mathrm{E}-02$ & 0.9588 \\
\hline $149-62$ & SF & 0.4161 & 1.0368 & 0.4314 & $0.23510 \mathrm{E}-02$ & 0.9656 \\
\hline $149-63$ & $\mathrm{DF}$ & 0.4879 & 1.0444 & 0.5096 & $0.26099 \mathrm{E}-02$ & 0.9075 \\
\hline $149-64$ & SF & 0.4294 & 1.0049 & 0.4315 & $0.24135 \mathrm{E}-02$ & 0.9911 \\
\hline $149-65$ & $\mathrm{SF}$ & 0.4358 & 1.0368 & 0.4518 & $0.24997 E-02$ & 0.9802 \\
\hline $149-66$ & DF & 0.5010 & 1.0444 & 0.5232 & $0.27825 \mathrm{E}-02$ & 0.9422 \\
\hline $149-67$ & SF & 0.4372 & 1.0049 & 0.4393 & $0.25154 \mathrm{E}-02$ & 1.0145 \\
\hline $149-68$ & $\mathrm{SF}$ & 0.4129 & 1.0368 & 0.4281 & $0.24575 \mathrm{E}-02$ & 1.0172 \\
\hline $149-69$ & DF & 0.4598 & 1.0444 & 0.4802 & $0.25035 \mathrm{E}-02$ & 0.9237 \\
\hline $149-70$ & SF & 0.3374 & 1.0049 & 0.3391 & $0.20904 \mathrm{E}-02$ & 1.0924 \\
\hline $149-71$ & $\mathrm{SF}$ & 0.3167 & 1.0368 & 0.3284 & $0.18772 E-02$ & 1.0130 \\
\hline $149-72$ & DF & 0.3291 & 1.0444 & 0.3437 & $0.17229 E-02$ & 0.8882 \\
\hline $149-73$ & $\mathrm{SF}$ & 0.2259 & 0.9845 & 0.2224 & $0.11928 E-02$ & 0.9503 \\
\hline
\end{tabular}

${ }^{a} \mathrm{SF}$ is single fuel column, $\mathrm{DF}$ is double fuel column, $\mathrm{B}$ is blanket and $R$ is reflector.

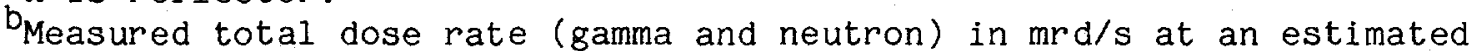
reactor power of 1 watt. Statistical uncertainties $2 \%$.

${ }^{c}$ Nodal transport calculation in xyz geometry with fluxes normalized to 8 watts, calculated doses in rads. For comparison with measurement the calculated doses are normalized by the mean $\mathrm{C} / \mathrm{E}$ for ${ }^{239} \mathrm{Pu}$ fission from 62 foil measurements in the core region. 
TABLE 7.7 Measured and Calculated Dose-Rates in the Axial Direction in Matrix 149-53

\begin{tabular}{|c|c|c|c|c|c|}
\hline $\begin{array}{c}\text { Axial } \\
\text { Displacement }(\mathrm{mm})\end{array}$ & Exp. ${ }^{a}$ & $\begin{array}{c}\text { Cell } \\
\text { Factor } \\
\end{array}$ & $\begin{array}{c}\text { Corrected } \\
\text { Exp. } \\
\end{array}$ & Calc. $b$ & $\mathrm{C} / \mathrm{E}$ \\
\hline 76.2 & 0.1600 & 0.9993 & 0.1599 & $0.87177 \mathrm{E}-03$ & 0.9661 \\
\hline 127.0 & 0.1965 & 0.9993 & 0.1964 & $0.11017 \mathrm{E}-02$ & 0.9941 \\
\hline 228.6 & 0.3523 & 1.0368 & 0.3653 & $0.20115 \mathrm{E}-02$ & 0.9758 \\
\hline 330.2 & 0.3400 & 1.0368 & 0.3525 & $0.20730 \mathrm{E}-02$ & 1.0420 \\
\hline 431.8 & 0.3051 & 1.0368 & 0.3163 & $0.173443-02$ & 0.9715 \\
\hline 584.2 & 0.1005 & 1.0000 & 0.1005 & $0.54986 \mathrm{E}-03$ & 0.9694 \\
\hline 685.8 & 0.0638 & 1.0000 & 0.0638 & $0.34260 \mathrm{E}-03$ & 0.9515 \\
\hline
\end{tabular}

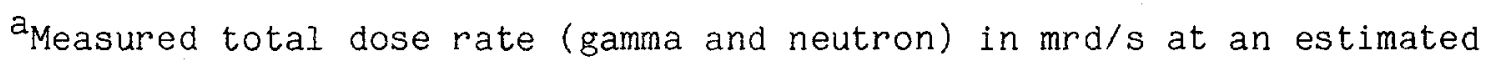
reactor power of 1 watt. Statistical uncertainties $2 \%$.

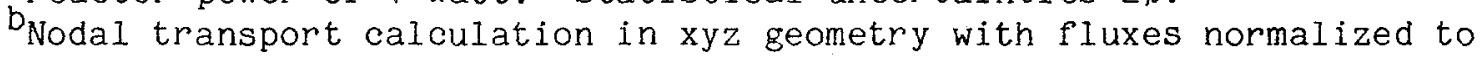
8 watts, calculated doses in rads. For comparison with measurement the calculated doses are normalized by the mean $\mathrm{C} / \mathrm{E}$ for ${ }^{2{ }^{39} \mathrm{Pu}} \mathrm{f}$ ission from 62 foil measurements in the core region. 
TABLE 7.8 Measured and Calculated Dose Rates in the Axial Direction in Matrix 149-60

\begin{tabular}{|c|c|c|c|c|c|}
\hline $\begin{array}{c}\text { Axial } \\
\text { Displacement }(\mathrm{mm}) \\
\end{array}$ & Exp. ${ }^{a}$ & $\begin{array}{c}\text { Cell } \\
\text { Factor } \\
\end{array}$ & $\begin{array}{c}\text { Corrected } \\
\text { Exp. } \\
\end{array}$ & Calc. ${ }^{b}$ & $C / E$ \\
\hline 76.2 & 0.1781 & 0.9993 & 0.1780 & $0.10129 \mathrm{E}-02$ & 1.0084 \\
\hline 127.0 & 0.2257 & 0.9993 & 0.2255 & $0.12830 E-02$ & 1.0079 \\
\hline 228.6 & 0.4592 & 1.0444 & 0.4796 & $0.24484 E-02$ & 0.9046 \\
\hline 330.2 & 0.4544 & 1.0444 & 0.4746 & $0.24471 \mathrm{E}-02$ & 0.9137 \\
\hline 431.8 & 0.3823 & 1.0444 & 0.3993 & $0.20229 E-02$ & 0.8977 \\
\hline 584.2 & 0.1016 & 1.0000 & 0.1016 & $0.57995 \mathrm{E}-03$ & 1.0114 \\
\hline 685.8 & 0.0640 & 1.0000 & 1.0640 & $0.35406 \mathrm{E}-03$ & 0.9802 \\
\hline
\end{tabular}

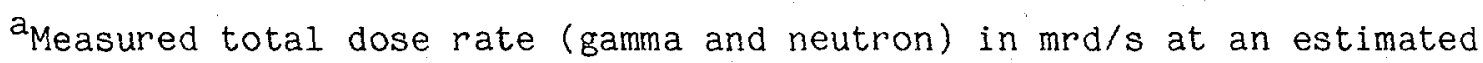
reactor power of 1 watt. Statistical uncertainties $2 \%$.

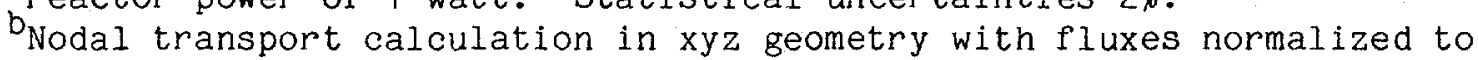
8 watts, calculated doses in rads. For comparison with measurement the calculated doses are normalized by the mean $C / E$ for ${ }^{23{ }^{9}} \mathrm{Pu}$ fission from 62 foil measurements in the core region. 
TABLE 7.9 Measured and Calculated Dose Rates in the Axial Direction in Matrix 149-70

\begin{tabular}{|c|c|c|c|c|c|}
\hline $\begin{array}{c}\text { Axial } \\
\text { Displacement }(\mathrm{mm}) \\
\end{array}$ & Exp. ${ }^{a}$ & $\begin{array}{c}\text { Cell } \\
\text { Factor } \\
\end{array}$ & $\begin{array}{c}\text { Corrected } \\
\text { Exp. } \\
\end{array}$ & Calc. ${ }^{b}$ & $\mathrm{C} / \mathrm{E}$ \\
\hline 76.2 & 1.0049 & 1.0049 & 0.4225 & $0.23253 E-02$ & 0.9753 \\
\hline 127.0 & 0.3889 & 1.0049 & 0.3908 & $0.22681 \mathrm{E}-02$ & 1.0283 \\
\hline 228.6 & 0.3374 & 1.0049 & 0.3391 & $0.20904 \mathrm{E}-02$ & 1.0924 \\
\hline 330.2 & 0.3112 & 1.0049 & 0.3127 & $0.18131 E-02$ & 1.0273 \\
\hline 431.8 & 0.2407 & 1.0049 & 0.2419 & $0.13880 \mathrm{E}-02$ & 1.0168 \\
\hline 584.2 & 0.0744 & 1.0000 & 0.0744 & $0.40232 E-03$ & 0.9581 \\
\hline 685.8 & 0.0423 & 1.0000 & 0.0423 & $0.23585 E-03$ & 0.9879 \\
\hline
\end{tabular}

\footnotetext{
a Measured total dose rate (gamma and neutron) in $\mathrm{mrd} / \mathrm{s}$ at an estimated reactor power of 1 watt. Statistical uncertainties $2 \%$.

$\mathrm{b}_{\text {Nodal }}$ transport calculation in $x y z$ geometry with fluxes normalized to 8 watts, calculated doses in rads. For comparison with measurement the calculated doses are normalized by the mean $\mathrm{C} / \mathrm{E}$ for ${ }^{239} \mathrm{Pu}$ fission from 62 foil measurements in the core region.
} 


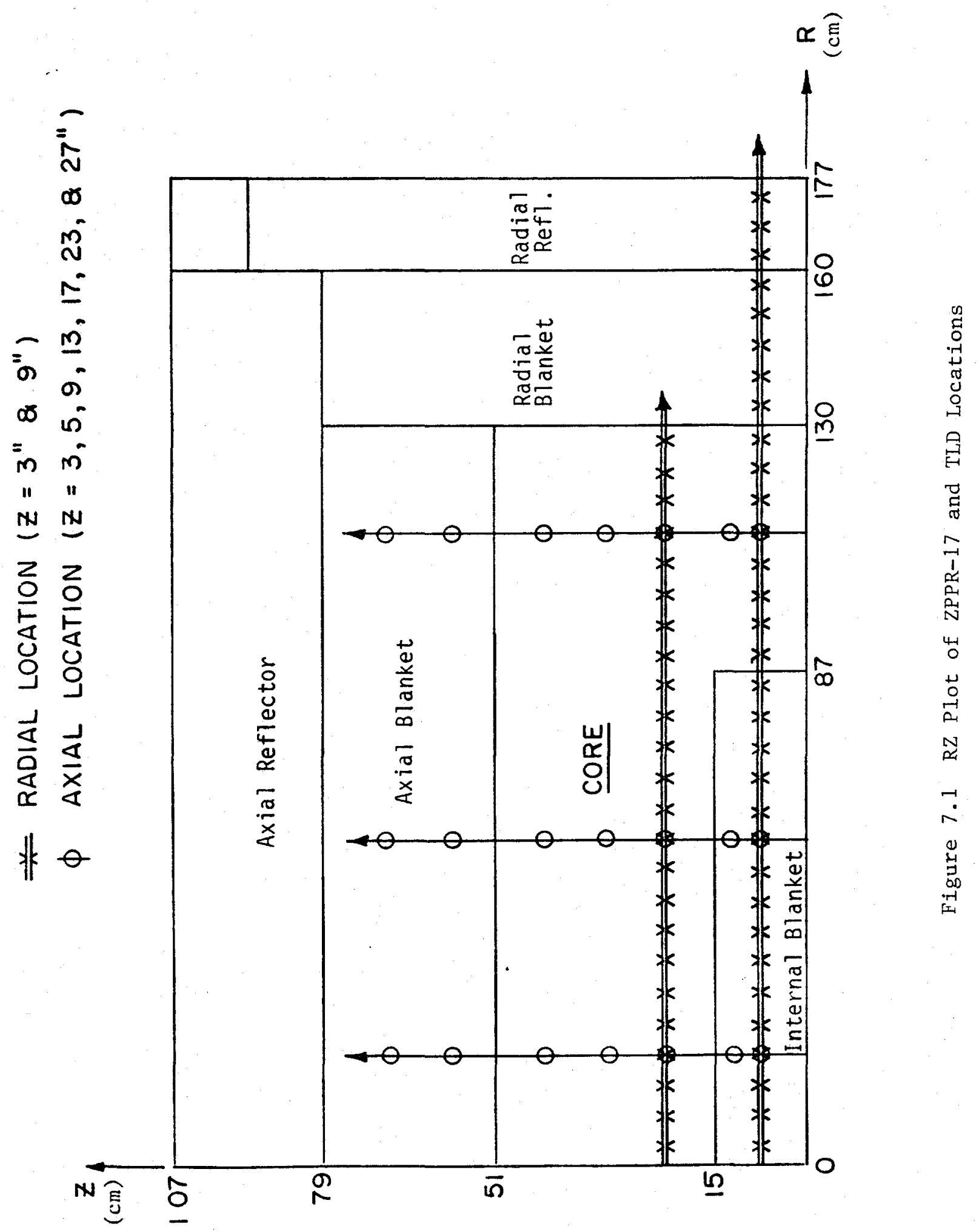




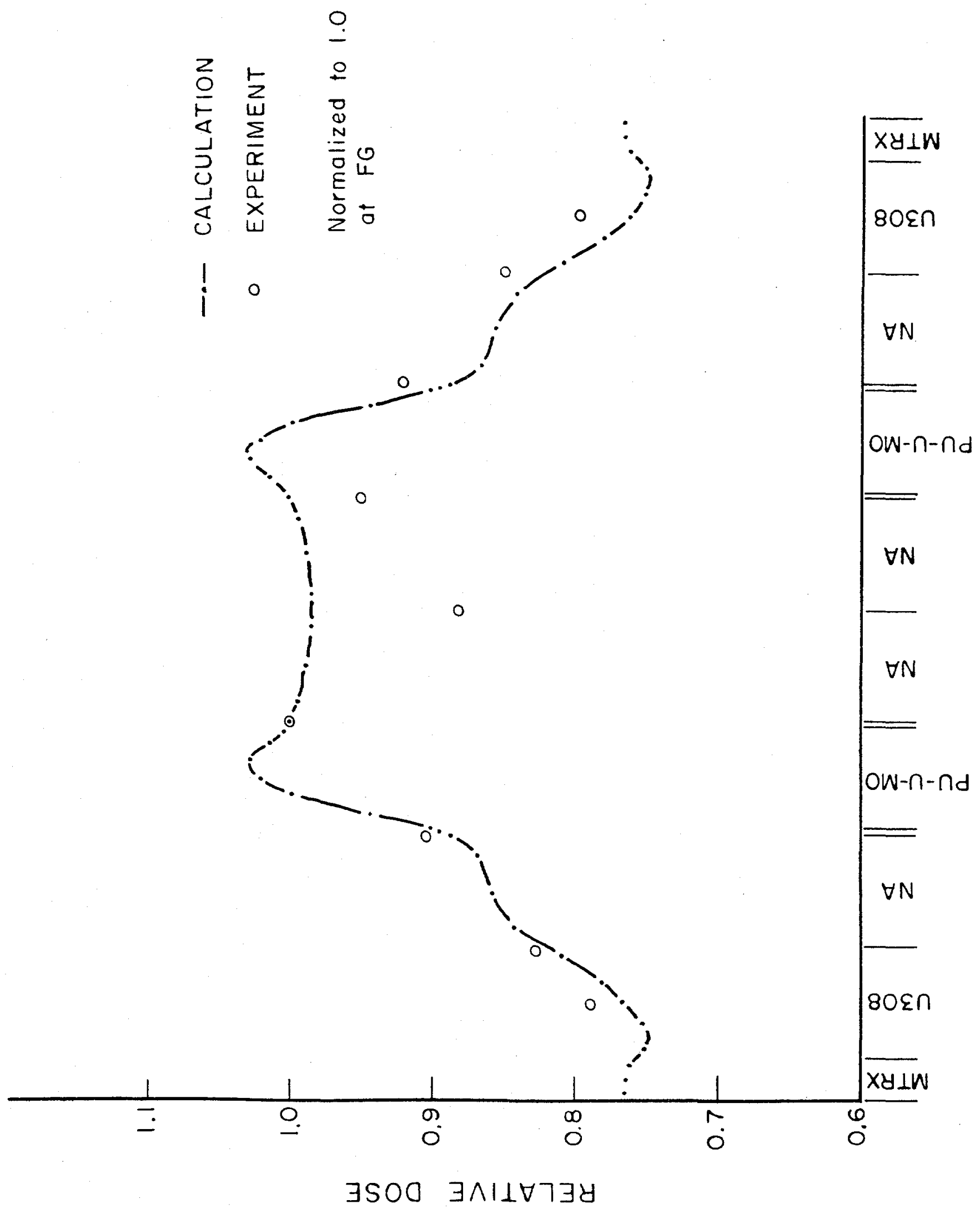




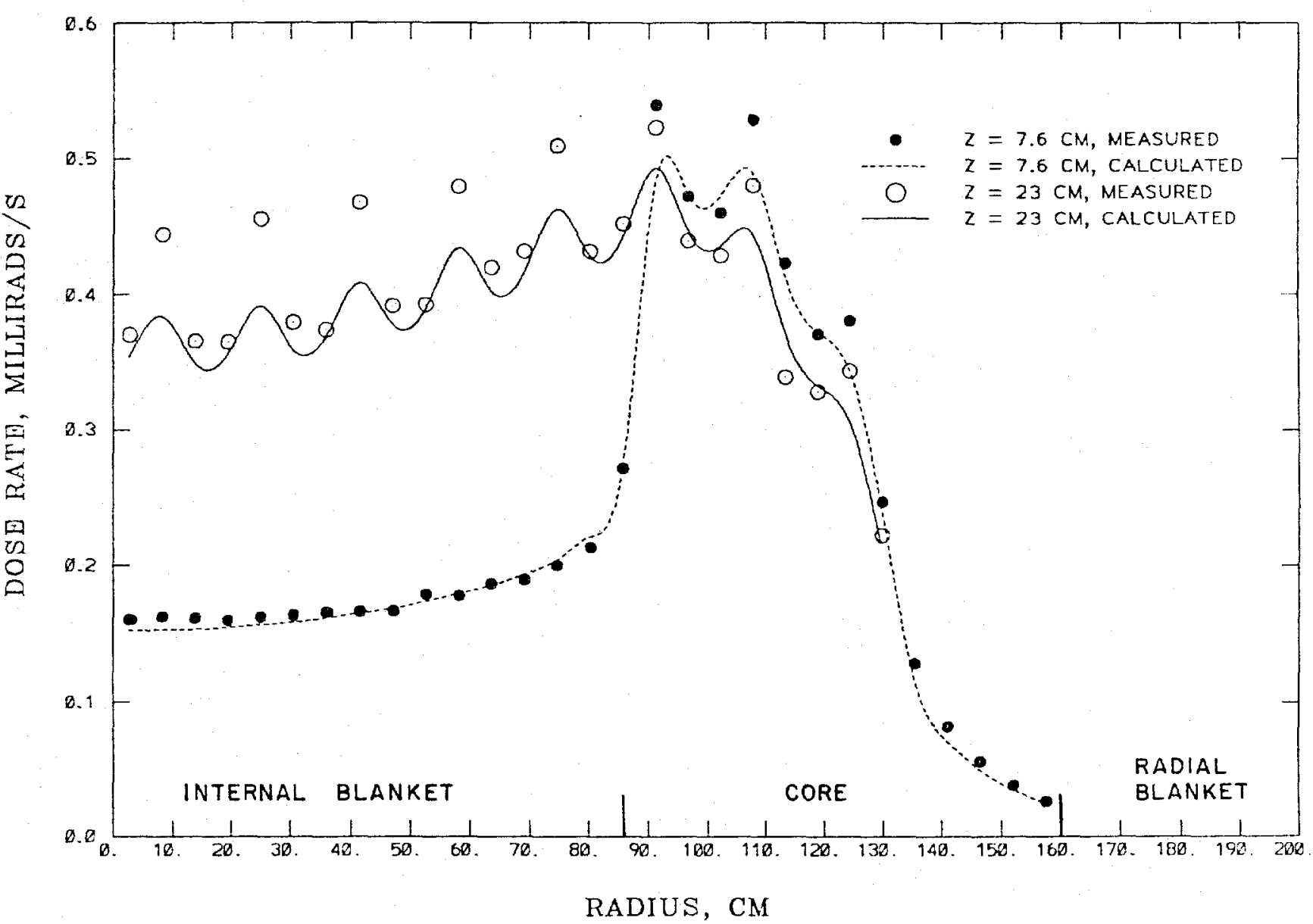

Fig. 7.3 Measured and Calculated Total Dose Rates Along the x-axis of ZPPR-17A. 


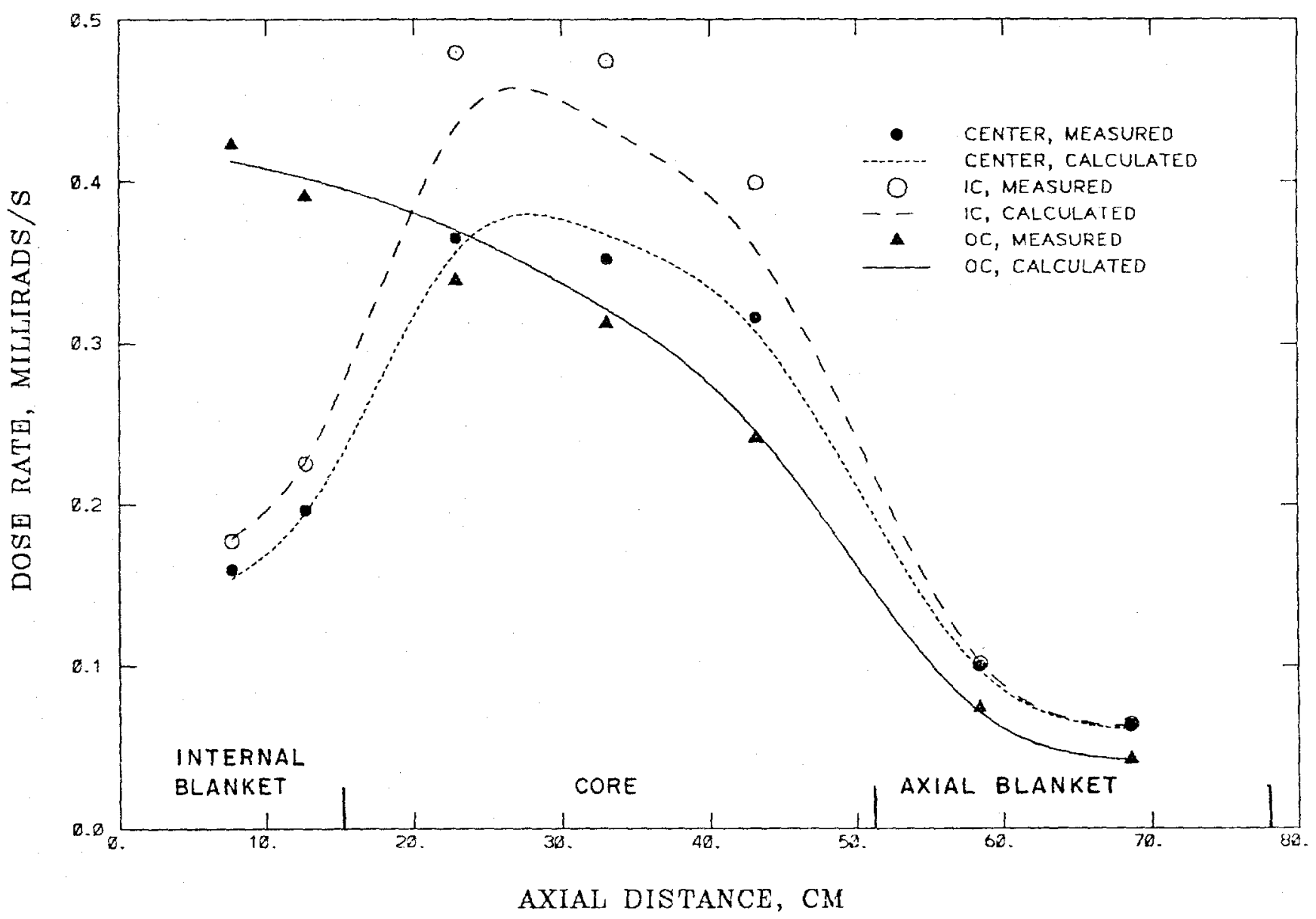

Fig. 7.4 Measured and Calculated Axial Distributions of Total Dose Rates in ZPPR-17A. 


\section{CONTROL ROD ANALYSIS}

Control rod measurements in ZPPR-17 were selected to cover different design options for the axially heterogeneous core. Measurements in ZPPR-17A, which did not contain CRPs in its reference configurations, focused on the worth of a single, central rod as a function of rod size, geometry and composition. Worths of CRP banks and one special four-rod bank were also measured. All measurements in ZPPR-17A were made with respect to fuel.

Worth of control rod banks, combinations of banks and banks with missing rods were measured in $Z P P R-17 B$ and $17 \mathrm{C}$. All these measurements were with respect to CRPS.

All data processing was done with the McCRUNCH code and all results presented in this section used the LSFIT option in McCRUNCH. The reference control rod positions and the locations of the special bank measured in $17 \mathrm{~A}$ are shown in Fig. 8.1.

Calculations of detector efficiencies and effective source ratios required for processing experimental data may usually be made to sufficient accuracy by two-dimensional xy models. However, the internal blanket of ZPPR-17 caused strong variations in the axial buckling as a function of radius, especially around the edge of the internal blanket. To avoid complication of the model, all calculations were made with a threedimensional xyz model with the cross sections collapsed to six energy groups. The data were collapsed from a 21-group xyz mode1. Six-group cross sections were obtained for the internal blanket, for single- and doublefuel-column drawers in the inner core and in the outer core, for the radial blanket, radial reflector, axial blanket and axial reflector. Source-driven calculations for the real flux were made for cases with reactivity below one dollar subcritical. Cases with reactivity closer to critical used a homogeneous flux solution. 


\subsection{Results for $2 P P R-17 \mathrm{~A}$}

The ZPPR-17A measurements included both plate- and pin-type rods using both natural and enriched $B_{4} C$. Rod size was mostly two-by-two drawers, but rods two-by-three and three-by-three drawers in size were measured. The additional drawers in the two-by-three rod were 1,248-48 and 1,249-48. Added to these for the three-by-three rod were 1,250-48, 1,250-49 and $1,250-50$.

Some pin-type rods used pin calandria and some had the $B_{4} C$ pellets tightly packed in drawers. The loading pattern of the tightly packed rod is given in Fig. 8.2. Measured results and $\mathrm{C} / \mathrm{E}$ values from $\mathrm{ZPPR}-17 \mathrm{~A}$ were reported in reference 2. Atom densities for the various rod types are given in Table 7.2 of reference 1.

Six-group cross sections for control rods and CRPs were generated for a model with one of the ZPPR-17B rod patterns: center and inner $r$ ing control rods, middle ring CRPs, odd-numbered control rods (15-25) and even numbered CRPs (14-24) in the outer ring. For all central rods, data processing was performed using detector efficiencies and source ratios calculated for the $100 \%$ natural $\mathrm{B}_{4} \mathrm{C}$ rod. These values were used in the MCCRUNCH code to process worths for all central and near-central rod variations. All central and near-central CRPs used calculated values for the central two-by-two CRP. Because of these approximations, C/E values are only produced for the central $\mathrm{CR}$ and the central CRP. Table 8.1 gives the analysis results for the central $C R$ and $C R P$, the special bank of $C R s$ and CRPs and reference CRP banks.

\subsection{Results for ZPPR-17B}

In $Z P P R-17 \mathrm{~B}$, a series of 20 control worth measurements was made. These included each of the rod banks, the inner and outer banks halfinserted, 8 different combinations of rod banks and 6 configurations with a rod missing from one of the banks representing stuck rod or rod run-out events. Generally, the central rod plus either the 12 outer-ring rods (numbers 14 to 25 ) or 6 outer ring rods (15 to 25 odd-numbered) were 
designated as operating (regulating) rods. The inner ring were startup rods and the middle ring or the middle ring plus the even-numbered rods in the outer ring were the secondary (shutdown) rods.

As in the case for ZPPR-17A, the 17B six-group cross sections were obtained for the internal blanket, for single- and double-fuel-column drawers in the inner core and in the outer core, for the radial blanket, radial reflector, axial blanket and axial reflector. Cross sections for CRPs were also obtained with this model. A second calculation with the center rod and 12 outer rods inserted was used to collapse data for the control rods.

The calculations were made in $x y z$ geometry with full-xy/half-z, quarter $-x y / h a l f-z$, and quarter $x y / f u l l-z$ symmetry as required to represent the geometry of the rod patterns. For the subcritical states, both an adjoint flux for the homogeneous problem and a source-driven real flux were required to calculate detector efficiencies and effective source ratios. All calculations were made with the finite-difference solution of DIF3D.

The measured values and $C / E$ results are given in Tables $8.2,8.3$ and 8.4 , grouped into rod banks, rod bank combinations and banks with missing rods. A shorthand notation $(a, b, c, d)$ is used to describe the number of control rods in the center position, the inner ring, the middle ring and the outer ring. Where necessary, the letter $\mathrm{H}$ is used to indicate rods half inserted and 0 is used to indicate odd-numbered rods. Worths are given as positive numbers for simplicity.

Greater detail of the measurements and data processing are given in reference 4.

The $C / E$ results are in the range of 0.95 (center rod) to 1.00 (outer ring half-inserted). No clear trend in $\mathrm{C} / \mathrm{E}$ as a function of radius emerges from the present analysis. The center rod is predicted 2-3\% lower than the inner ring $(C / E=0.98)$ the middle $r i n g ~ C / E$ is 0.97 and the outer ring $C / E$ is 0.99 . 
Predictions for rod banks combinations and banks with missing rods are consistent with those for the individual banks with $C / E$ values in the range 0.97 to 1.00 .

The ratios of worths for rod banks half-inserted to the worths of the fully inserted banks are quite interesting:

$\begin{array}{lll}\text { inner ring } & \text { measured } & 0.545 \\ & \text { calculated } & 0.542 \\ & & \\ \text { outer ring } & \text { measured } & 0.478 \\ & \text { calculated } & 0.482\end{array}$

Thus, the rods half-inserted in the internal blanket are worth $9 \%$ more than half of the worth when fully-inserted while at the periphery of the blanket they are worth 4\% less than half of the worth when fully-inserted. Experiment and calculation agree closely for these ratios.

Some rod interaction effects are shown in Table 8.5 which compares the worth of the center rod and of different banks of rods when inserted alone or with other rod banks inserted in the core. These worths were obtained by subtraction of the worths in the previous tables, hence the statistical uncertainties increase. The worth of the center rod, $1 \$$ when inserted alone, varies from $0.22 \$$ with the inner and middle rings inserted to $2.14 \$$ with the outer ring inserted.

The worths of withdrawal of single rods from a bank in different situations are compared in Table 8.6. Since the single rod worths were not measured, the average worth of the rods in the appropriate banks are given for comparison. The worths of the withdrawn rods vary considerably: the worth of CR2 in the inner bank is $0.46 \$$ when the center and middle banks are inserted and $0.94 \$$ when the center and 6 outer bank rods are inserted compared with the mean worth in the bank of $0.97 \$$. The worth of CR14 in the outer bank (with center rod also inserted) is $4.1 \$$ compared with the mean worth in the outer bank of 1.9\$. These interaction effects are calculated within $10 \%$ by diffusion theory. 
Calculations for the symmetric rod banks have been made by nodal diffusion (NDT) and nodal transport (NTT) models. The results are compared with the finite difference diffusion (FDDT) values, using a $55 \mathrm{~mm}$ mesh size, in Table 8.7. Mesh and transport effects are quite different to those found in conventional and radially-heterogeneous cores. Mesh corrections are relatively large - from $+8 \%$ to $+12 \%$ - while the transport correction is near zero for the central rod increasing with radius to $-5 \%$ for the outer rod bank. The calculated worths are notably higher ( $6 \%$ to $9 \%$ ) by nodal transport calculation then the original finite difference diffusion.

The nodal transport $\mathrm{C} / \mathrm{E}$ results show no clear trend with radius but the center rod and the middle bank have C/Es about $1 \%$ lower than those for the inner bank and the outer bank. The C/E results for bank combinations fall between those for the individual banks. The spread in $\mathrm{C} / \mathrm{E}$ values ( 1.041 to 1.057 ) is less than half of the spread with the finite difference diffusion calculations.

\subsection{Results for $2 P P R-17 \mathrm{C}$}

The ZPPR-17C reference core simulated a beginning-of-cycle loading with primary control rods -- center and 12 outer ring -- half inserted. The control rod worth measurements provide data on the worths of full insertion of secondary control banks in this loading. The secondary rod locations were the six inner ring (IR) positions and the six middle ring (MR) positions. The basic rod banks were six inner ring (6IR), six middle ring (6MR), 3IR, 3MR and a combination 3IR + 3MR. The three-rod patterns were measured both for the rods symmetrically disposed around the hexagonal bank and for the three rods in adjacent locations. Several cases of banks with missing rods were also measured, $5 I R, 5 M R, 3 I R+2 M R$ and $2 I R+2 I R+3 M R$.

Three of the rod patterns, 6IR, 6MR and 5MR, were also measured in ZPPR-17B, the end-of-cycle simulation with all rods withdrawn. These cases provide a comparison of the interaction effects and of the accuracy of calculation of rod worths in an operating condition rather than in a reference core with no rods inserted. 
The six-group data used the microscopic cross sections produced for $17 \mathrm{~B}$ and were not separately collapsed for 17C. Only two of the control configurations permitted quarter-xy symmetry to be used, six cases required full-xy representation while two cases were symmetric about the $y$-axis and one was symmetric about the x-axis. All configurations were calculated with the full-xy model to avoid proliferation of models.

Since the control rod worths were measured with the rods fully inserted, the model used half-z symmetry. Shielding factors for the halfinserted rods in the reference core, 0.292 for the CRs and 0.708 for the CRPs, were used to adjust the k-effective in this model to 0.9888 . The keffective for the reference core using a quarter-xy full-z model was 0.989323. The two control patterns with quarter-xy symmetry ( 6 inner ring and 6 middle ring) were also calculated with the full-z model to check the results from the approximate models.

Calculations of detector efficiencies and effective source worths were made with the finite-difference diffusion path of DIF3D. Calculated worths for the two symmetric rod banks were also obtained with the nodal diffusion and nodal transport solutions.

Additional details of the measurements and data processing are given in reference 4 .

Measured worths and $C / E$ values are given in Table 8.8 . Statistical and correlated uncertainty components are given to three figures to facilitate derivation of uncertainties when subtracting configuration worths. The calculated worths in Table 8.8 are obtained from the half-z model.

The C/E results vary by only $3 \%$ over the range of the configurations. The highest values are for the three adjacent rods in the inner and middle ring. In both bases, these are predicted $2 \%$ higher than their counterparts which have three rods symmetrically disposed around the ring. Apart from these two cases, the $C / E$ values are in the range 0.949 to 0.963 . 
Rod interaction effects are shown in Table 8.9 in terms of the worth per rod (WPR) in banks of $6,5,3$ and 2 rods. The inner ring rods show a negative interaction (WPR for the 3 symmetric rods is $15 \%$ higher than for the bank of 6 rods or 3 adjacent rods). The wider-spaced rods in the middle ring show a more neutral interaction (WPR for the 3 symmetric rods is the same as that for the bank of 6 rods, but 25\% greater than for 3 adjacent rods). The worth of either bank of 3 rods is suppresed by $20 \%$ when the other bank is also present. These latter effects are predicted with a C/E bias similar to that for the individual banks.

Improved calculations have been made for the complete inner and middle banks of rods which were symmetric in the $x y$-plane. These calculations used full z representation to model the half-inserted rods in the reference loading. Calculations were made with finite-difference diffusion (FDDT), with nodal diffusion (NDT) which has a small mesh-size error and with nodal transport (NTT). The calculated rod bank worths are compared in Table 8.10 .

The effects on k-effective due to using the half-z model vary a little between the reference and the control bank cases and result in changes in rod worths of $0.5 \%$ and $0.7 \%$. Corrections to rod worths for diffusion theory mesh size are $+10 \%$ and $+11 \%$ while transport corrections are $-0.6 \%$ and $-2 \%$. The mesh corrections are considerably larger than found in other cores. Similar mesh and transport corrections were found for ZPPR-17B. Thus the effects are due to the internal blanket rather than the rods which were half-inserted in the core.

The worths of the inner bank, the middle bank and five rods in the middle bank were measured both in ZPPR-17B and in ZPPR-17C. The experimental worths and predictions are compared in Table 8.11. The worth of the inner bank is similar in either core but the worth of the middle bank is depressed by $8 \%$ in $17 \mathrm{C}$ due to the half-inserted outer bank. The $\mathrm{C} / \mathrm{E}$ results are consistent between $17 \mathrm{~B}$ and $17 \mathrm{C}$ within the (total) uncertainties of the experiments of about $0.9 \%$. 


\subsection{Axial Worth Profiles for $\mathrm{B}_{4} \mathrm{C}$}

The worth profile for a central control rod was measured in both ZPPR-17B and 17C. In 17B, the profile was measured for a $1.02 \mathrm{~m}$-long, twoby-two-drawer $\mathrm{B}_{4} \mathrm{C}$ column inserted in $102 \mathrm{~mm}$ steps by rearranging the contents of the control rod drawers. There was a separate reactor run for each step. Worths for the various steps were calculated with nodal xyz diffusion methods using the six-group cross section data. The axial node spacings in the model were adjusted so that the leading edge of the control rod corresponded to a node. Calculated and measured worths are shown in Fig. 8.3. The calculated values are normalized to the average $\mathrm{C} / \mathrm{E}$ value and reproduce the measured profile within $1 \%$ except for the initial, small insertion.

In addition to these static measurements, the worth profile in $17 \mathrm{~B}$ was measured for a $1.02 \mathrm{~m}_{\text {-long }} \mathrm{B}_{4} \mathrm{C}$ segment using the long drawer technique. Reactivity values were derived for various axial positions as the $B_{4} C$ column was driven from $102 \mathrm{~mm}$ above the core/axial blanket interface until the column exactly filled the core region. Values were obtained for several in-out cycles. The long drawer segments were in 149-49 and 249-49 and the other three drawers in the central control position contained their standard sodium-filled drawers.

A third measure of the axial worth profile of boron was obtained using the axial tube small sample oscillator technique. This measurement was made in ZPPR-17A in location 159-49, in the region containing internal blanket but well away from the core center. The enriched boron sample was a cylinder $55 \mathrm{~mm}$ long and $10.2 \mathrm{~mm}$ in diameter with a ${ }^{10} \mathrm{~B}$ mass of $3.65 \mathrm{~g}$. In order to compare with the other techniques, the small sample reactivities were integrated over the core height.

Results from the three profile measurements are shown in

Fig. 8.4. Note that rod position zero corresponds to full insertion. The reactivity values are those from the long-drawer measurement. Reactivities from the other measurements were normalized to the long-drawer value at full 
insertion to allow the worth profiles to be compared. As Fig. 8.4 shows, the three methods give essentially the same shape, even with ${ }^{10} \mathrm{~B}$ sample mass variation from $3.65 \mathrm{~g}$ in the small sample experiment to $735.6 \mathrm{~g}$ in the static experiment. This implies that the axial flux tilt caused by the boron is not large. These results also show that calculated worth profiles can be verified by comparing with any of the three measured profiles.

Details of the $17 \mathrm{~B}$ axial boron measurements are given in reference 2.

The long-drawer measurement was repeated in two locations in ZPPR-17C. In the first location, 149-49 and 249-49, the central, halfinserted control rod was removed and replaced with the long-drawer (1.02 m-long $\mathrm{B}_{4} \mathrm{C}$ column) and CRP drawers elsewhere. The 12 half-inserted, outer-ring rods were in place. The second location was in 1,265-49, an outermost location with internal blanket. In this second case, all 13 halfinserted rods were in place. The results for these two measured profiles are given in Figs. 8.5 and 8.6. Again, rod position zero corresponds to full insertion. The difference in axial profiles can be seen with a smaller slope in the internal blanket for the core-center measurement. The axial asymmetry of the core is seen for both locations in the smaller slope in the axial portion with the inserted control rods (higher values of rod position).

Details of the $17 \mathrm{C}$ axial boron measurements are given in reference 5 .

\subsection{Additional Calculations for ZPPR-17B}

The reference calculations for $Z P P R-17$ used finite difference diffusion since this model was used to provide detector efficiencies and source ratios for experimental data processing. More refined calculations for a number of cases using nodal diffusion and nodal transport methods are shown for $17 \mathrm{~B}$ in Table 8.7 and for $17 \mathrm{C}$ in Table 8.10. Additional calculations were made for the principal rod bank worths in ZPPR-17B. 
The effects of group collapse were estimated by calculating the bank worths by nodal diffusion with 21 group cross sections. The results are compared with those from the six group calculations in Table 8.12. Group condensation effects were larger then expected from results in other cores and varied with position from $-7 \%$ for the central rod to $-4 \%$ for the outer rod bank. These corrections increase the radial discrepancy in $\mathrm{C} / \mathrm{E}$ by 3 to $4 \%$.

The rod worths were also calculated using the TRITAC code in xzy geometry and 54 quadrature. The results are given in Table 8.13. Corrections for anisotropic transport, not treated in the TRITAC code, were calculated using the nodal transport code. These varied from $-1.8 \%$ for the central control rod to $+0.5 \%$ for the outer bank. The TRITAC worths, after adjustment for streaming, were lower than the nodal transport worths. The difference varied with radius from $-4 \%$ for the central rod to $-0.6 \%$ for the outer bank.

Table 8.14 compares the rod bank worth C/Es for ZPPR-17B by different calculation methods, after the group condensation corrections have been supplied. The radial variation in $\mathrm{C} / \mathrm{E}$ is about $10 \%$ and twice that found in the analysis of radial fission rates. 
TABLE 8.1 Recommended Control and CRP Worths in ZPPR-17A and Comparison with Calculation

\begin{tabular}{|c|c|c|c|c|c|}
\hline Case & $\begin{array}{c}\text { Experimental } \\
\text { Worth, } \$ \\
\end{array}$ & $\begin{array}{r}\text { Total } 10 \\
\text { Uncertainty, } \% \\
\end{array}$ & $\begin{array}{c}\text { Calcul ated } \\
\mathrm{k}_{\mathrm{eff}} \\
\end{array}$ & $\begin{array}{l}\text { Calculated } \\
\text { Worth, } \$^{\mathrm{b}} \\
\end{array}$ & $\mathrm{C} / \mathrm{E}$ \\
\hline Central $\mathrm{CR}$ & 1.073 & 0.88 & 0.984803 & 1.044 & 0.973 \\
\hline 4 CRs & 3.347 & 0.87 & 0.977174 & 3.386 & 1.012 \\
\hline 4 CRPS & 0.588 & 0.92 & 0.986100 & 0.650 & 1.105 \\
\hline Central CRP & 0.0837 & 1.45 & 0.987935 & 0.0932 & 1.114 \\
\hline $\begin{array}{l}\text { Central } \\
6 \text { Inner CRPs }\end{array}$ & 1.054 & 0.89 & 0.984374 & 1.175 & 1.115 \\
\hline $\begin{array}{l}\text { Central } \\
6 \text { Inner } \\
6 \text { Middle CRPs }\end{array}$ & 1.776 & 0.88 & 0.981545 & 2.040 & 1.150 \\
\hline All $25 \mathrm{CRPS}$ & 6.649 & 0.87 & 0.963450 & 7.693 & 1.157 \\
\hline
\end{tabular}

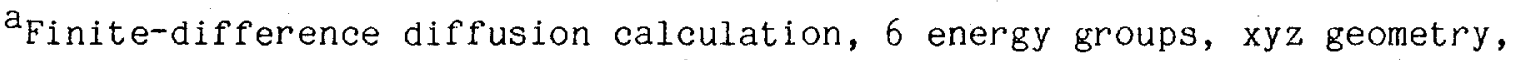
$5 \mathrm{~cm}$ mesh; Reference $\mathrm{k}_{\text {eff }}=0.988243$.

$b_{\text {Using }} \beta=0.3385 \%$. 
TABLE 8.2 Worths of Control Rod Banks in ZPPR-17B

\begin{tabular}{|c|c|c|c|c|c|c|c|}
\hline Step $^{a}$ & Control Rods ${ }^{\mathrm{b}}$ & $\begin{array}{c}\text { Experimental } \\
\text { Worth, } \$ \\
\end{array}$ & $\begin{array}{c}\text { Statistical } \\
10, \% \\
\end{array}$ & $\begin{array}{c}\text { Correlated } \\
10, \% \\
\end{array}$ & $\begin{array}{c}\text { Calculated } \\
k \text {-eff }\end{array}$ & $\begin{array}{l}\text { Calculated } \\
\text { Worth, } \$ \\
\end{array}$ & $C / E$ \\
\hline 1 & $\begin{array}{l}\text { Central CR } \\
(1,0,0,0)\end{array}$ & 1.001 & 0.087 & 0.811 & 0.982117 & 0.955 & 0.954 \\
\hline 3 & $\begin{array}{l}\text { IR } \\
(0,6,0,0)\end{array}$ & 5.822 & 0.116 & 0.866 & 0.966887 & 5.708 & 0.980 \\
\hline 2 & $\begin{array}{l}\text { IR Half Inserted } \\
(0,6 \mathrm{H}, 0,0)\end{array}$ & 3.171 & 0.123 & 0.865 & 0.975199 & 3.095 & 0.976 \\
\hline 10 & $\begin{array}{l}M R \\
(0,0,6,0)\end{array}$ & 8.318 & 0.121 & 0.859 & 0.959562 & 8.048 & 0.968 \\
\hline 18 & $\begin{array}{l}\mathrm{OR} \\
(0,0,0,12)\end{array}$ & 22.477 & 0.120 & 0.855 & 0.917563 & 22.186 & 0.987 \\
\hline 17 & $\begin{array}{l}\text { OR Half Inserted } \\
(0,0,0,12 \mathrm{H})\end{array}$ & 10.731 & 0.146 & 0.859 & 0.951408 & 10.695 & 0.997 \\
\hline
\end{tabular}

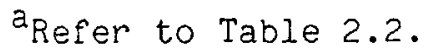

$b_{I R}=$ inner ring, $M R=$ middle ring, $O R=$ outer $r i n g, H=$ half-inserted.

${ }^{C}$ Finite difference diffusion, xyz geometry, 6 energy groups, reference k-effective 0.985234 .

$d_{E}$-effective $=0.3374 \%$. 
TABLE 8.3 Worths of Rod Bank Combinations in ZPPR-17B

\begin{tabular}{|c|c|c|c|c|c|c|c|}
\hline Step $^{a}$ & Control Rods $b$ & $\begin{array}{l}\text { Experimental } \\
\text { Worth, } \$ \\
\end{array}$ & $\begin{array}{c}\text { Statistical } \\
10, \% \\
\end{array}$ & $\begin{array}{c}\text { Correlated } \\
10, \% \\
\end{array}$ & $\begin{array}{c}\text { Calculated } \\
k \text {-eff }\end{array}$ & $\begin{array}{l}\text { Calculated } \\
\text { Worth, } \$^{d} \\
\end{array}$ & $\mathrm{C} / \mathrm{E}$ \\
\hline 4 & $\begin{array}{l}\text { Center + IR } \\
(1,6,0,0)\end{array}$ & 6.137 & 0.117 & 0.869 & 0.965778 & 6.060 & 0.987 \\
\hline 9 & $\begin{array}{l}\text { Center }+M R \\
(1,0,6,0)\end{array}$ & 9.017 & 0.126 & 0.861 & 0.957436 & 8.734 & 0.968 \\
\hline 19 & $\begin{array}{l}\text { Center }+ \text { OR } \\
(1,0,0,12)\end{array}$ & 24.589 & 0.118 & 0.852 & 0.912298 & 24.050 & 0.978 \\
\hline 6 & $\begin{array}{l}I R+M R \\
(0,6,6,0)\end{array}$ & 11.952 & 0.163 & 0.870 & 0.948020 & 11.809 & 0.988 \\
\hline 13 & $\begin{array}{l}I R+O R \text { odd } \\
(0,6,0,60)\end{array}$ & 17.856 & 0.147 & 0.858 & 0.931124 & 17.482 & 0.979 \\
\hline 5 & $\begin{array}{l}\text { Center + IR + MR } \\
(1,6,6,0)\end{array}$ & 12.169 & 0.158 & 0.874 & 0.947343 & 12.032 & 0.989 \\
\hline 14 & $\begin{array}{c}\text { Center + IR } \\
+ \text { OR odd } \\
(1,6,0,6 \varnothing)\end{array}$ & 18.279 & 0.137 & 0.861 & 0.929798 & 17.936 & 0.981 \\
\hline 16 & $\begin{array}{c}\text { Center + MR } \\
+ \text { OR odd } \\
(1,0,6,60)\end{array}$ & 20.490 & 0.113 & 0.857 & 0.923764 & 20.018 & 0.977 \\
\hline
\end{tabular}

a Refer to Table 2.2 .

${ }^{b}{ }_{I R}=$ inner ring, $M R=$ middle ring, $O R=$ outer $r i n g, \emptyset=$ odd numbered rods.

CFinite difference diffusion, xyz geometry, 6 energy groups, reference k-effective 0.985234 .

$d_{B \text {-effective }}=0.3374 \%$. 
TABLE 8.4 Worths of Rod Banks with Missing Rods in ZPPR-17B

\begin{tabular}{|c|c|c|c|c|c|c|c|}
\hline Step $^{a}$ & Control Rods ${ }^{b}$ & $\begin{array}{l}\text { Experimental } \\
\text { Worth, } \$ \\
\end{array}$ & $\begin{array}{c}\text { Statistical } \\
10, \% \\
\end{array}$ & $\begin{array}{c}\text { Correlated } \\
10, \% \\
\end{array}$ & $\begin{array}{c}\begin{array}{c}\text { Calculated } \\
\mathrm{k}-\mathrm{eff}^{\mathrm{c}}\end{array} \\
\end{array}$ & $\begin{array}{l}\text { Calculated } \\
\text { Worth, } \$^{\mathrm{d}} \\
\end{array}$ & $\mathrm{C} / \mathrm{E}$ \\
\hline 11 & $\begin{array}{l}M R-C R 8 \\
(0,0,5,0)\end{array}$ & 6.537 & 0.134 & 0.863 & 0.964752 & 6.387 & 0.977 \\
\hline 8 & $\begin{array}{l}\text { Center, IR-CR2, MR } \\
(1,5,6,0)\end{array}$ & 11.718 & 0.161 & 0.872 & 0.948817 & 11.546 & 0.985 \\
\hline 12 & $\begin{array}{l}\text { Center, IR-CR2, } \\
\text { OR odd } \\
(1,5,0,6 \emptyset)\end{array}$ & 17.321 & 0.131 & 0.860 & 0.932462 & 17.025 & 0.983 \\
\hline 7 & $\begin{array}{l}\text { Center, IR, MR-CR8 } \\
(1,6,5,0)\end{array}$ & 10.804 & 0.180 & 0.880 & 0.951187 & 10.768 & 0.997 \\
\hline 15 & $\begin{array}{l}\text { Center, MR-CR8, } \\
\text { OR odd } \\
(1,0,5,60)\end{array}$ & 18.625 & 0.161 & 0.860 & 0.928746 & 18.297 & 0.982 \\
\hline 20 & $\begin{array}{l}\text { Center, OR-CR14 } \\
(1,0,0,11)\end{array}$ & 20.435 & 0.170 & 0.862 & 0.922999 & 20.284 & 0.993 \\
\hline
\end{tabular}

a Refer to Table 2.2 .

$\mathrm{b}_{I R}=$ inner ring, $M R=$ middle ring, $O R=$ outer ring, $\theta=$ odd numbered rods .

${ }^{c}$ Finite difference, xyz geometry, 6 energy groups, reference k-effective 0.985234 .

$\mathrm{d}_{\text {Beffective }}=0.3374 \%$. 
TABLE 8.5 Control Rod Interaction Effects in ZPPR-17B

\begin{tabular}{|c|c|c|c|c|c|c|}
\hline $\begin{array}{l}\text { Control } \\
\text { Rods } \\
\end{array}$ & $\begin{array}{l}\text { From } \\
\text { Steps }\end{array}$ & $\begin{array}{l}\text { Rods Inserted } \\
\text { in Core } \\
\end{array}$ & $\begin{array}{c}\text { Experimental } \\
\text { Worth, } \$ \\
\end{array}$ & $\begin{array}{c}\text { Statistical } \\
\text { Uncertainty, } \%\end{array}$ & $\begin{array}{c}\text { Calculated } \\
\text { Worth, } \$\end{array}$ & $\mathrm{C} / \mathrm{E}$ \\
\hline $\mathrm{CR}$ & $\begin{array}{c}1 \\
6,5 \\
4,3 \\
14,13 \\
9,10 \\
19,18\end{array}$ & $\begin{array}{l}\text { None } \\
I R+M R \\
I R \\
I R+60 R \text { odd } \\
M R \\
O R\end{array}$ & $\begin{array}{l}1.001 \\
0.217 \\
0.315 \\
0.423 \\
0.699 \\
2.112\end{array}$ & $\begin{array}{r}0.1 \\
12.6 \\
3.1 \\
8.6 \\
2.2 \\
1.7\end{array}$ & $\begin{array}{l}0.955 \\
0.223 \\
0.352 \\
0.454 \\
0.686 \\
1.864\end{array}$ & $\begin{array}{l}0.954 \\
1.028 \\
1.117 \\
1.073 \\
0.981 \\
0.883\end{array}$ \\
\hline IR & 6,10 & $\begin{array}{l}\text { None } \\
\text { MR }\end{array}$ & $\begin{array}{l}5.823 \\
3.634\end{array}$ & $\begin{array}{l}0.1 \\
0.6\end{array}$ & $\begin{array}{l}5.708 \\
3.761\end{array}$ & $\begin{array}{l}0.980 \\
1.035\end{array}$ \\
\hline$M R$ & $\begin{array}{l}10 \\
6,3\end{array}$ & $\begin{array}{l}\text { None } \\
\text { IR }\end{array}$ & $\begin{array}{l}8.318 \\
6.130\end{array}$ & $\begin{array}{l}0.1 \\
0.3\end{array}$ & $\begin{array}{l}8.048 \\
6.101\end{array}$ & $\begin{array}{l}0.968 \\
0.995\end{array}$ \\
\hline 6 OR odd & $\begin{array}{l}13,3 \\
14,4 \\
16,9\end{array}$ & $\begin{array}{l}\text { IR } \\
\text { Center + IR } \\
\text { Center + MR }\end{array}$ & $\begin{array}{l}12.034 \\
12.142 \\
11.475\end{array}$ & $\begin{array}{l}0.2 \\
0.2 \\
0.2\end{array}$ & $\begin{array}{l}11.774 \\
11.876 \\
11.284\end{array}$ & $\begin{array}{l}0.978 \\
0.978 \\
0.983\end{array}$ \\
\hline
\end{tabular}


TABLE 8.6 Worth of Single Rods Withdrawn from Rod Banks in ZPPR-17B

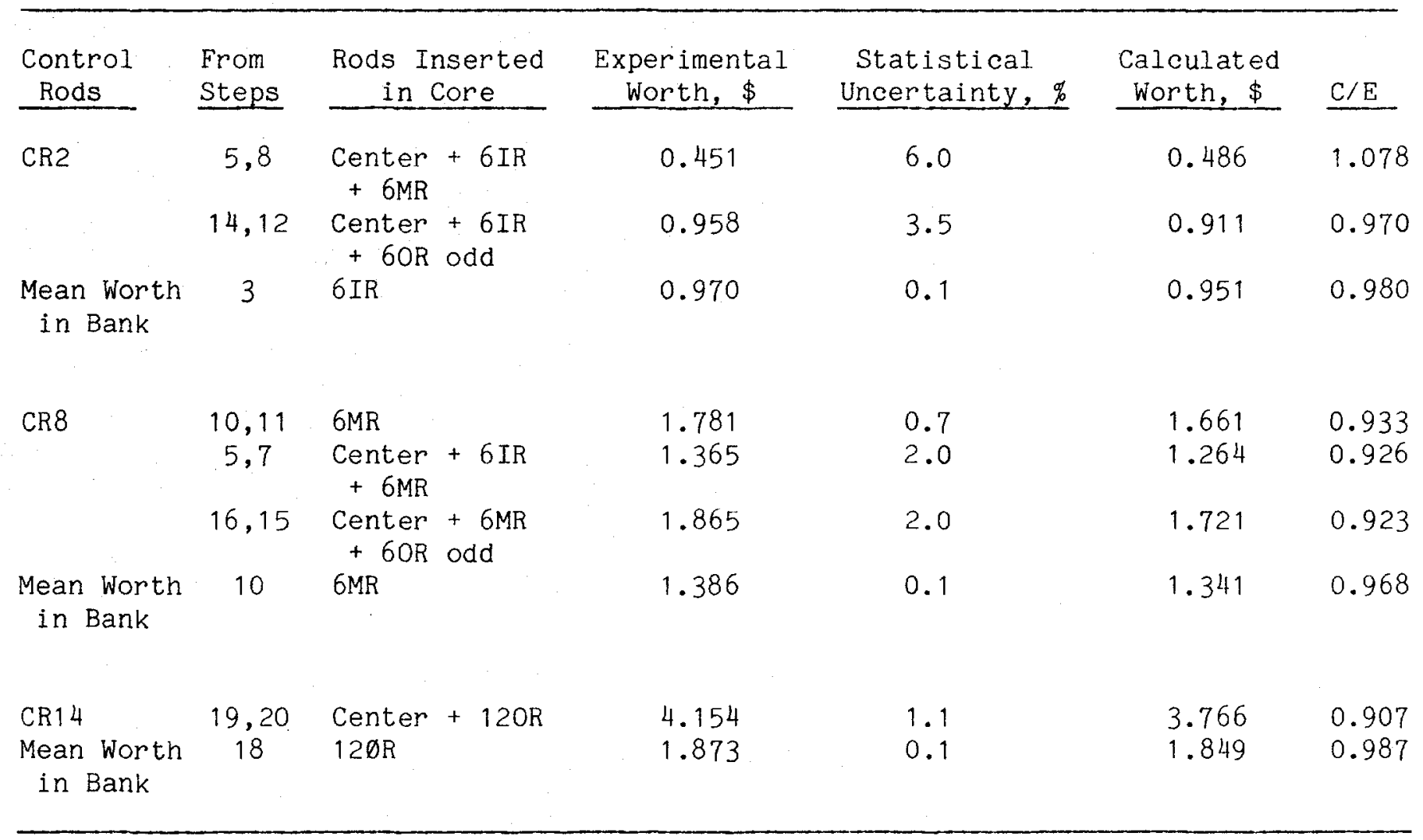


TABLE 8.7 Calculated Control Rod Worths in ZPPR-17B using Nodal Diffusion and Nodal Transport Models

\begin{tabular}{|c|c|c|c|c|c|c|}
\hline Control Rods & $\begin{array}{c}\text { FDDT } \\
\text { Worth, \$ }\end{array}$ & $\begin{array}{c}\text { NDT } \\
\text { Worth, \$ } \\
\end{array}$ & $\begin{array}{c}\text { Mesh } \\
\text { Correction, \% } \\
\end{array}$ & $\begin{array}{c}\text { NTT } \\
\text { Worth, \$ } \\
\end{array}$ & $\begin{array}{c}\text { Transport } \\
\text { Correction, } \$ \\
\end{array}$ & $\mathrm{C} / \mathrm{E}$ \\
\hline CR1 & 0.955 & 1.044 & +9.3 & 1.042 & -0.2 & 1.041 \\
\hline IR & 5.708 & 6.211 & +8.8 & 6.136 & -1.2 & 1.054 \\
\hline MR & 8.048 & 8.918 & +10.8 & 8.694 & -2.5 & 1.045 \\
\hline$\emptyset \mathrm{R}$ & 22.186 & 24.776 & +11.7 & 23.643 & -4.8 & 1.052 \\
\hline $\mathrm{CR} 1+\mathrm{IR}$ & 6.060 & 6.555 & +8.2 & 6.488 & -1.0 & 1.057 \\
\hline $\mathrm{CR} 1+\mathrm{MR}$ & 8.734 & 9.635 & +10.3 & 9.420 & -2.2 & 1.045 \\
\hline $\mathrm{CR} 1+\emptyset \mathrm{R}$ & 24.050 & 26.966 & +12.1 & 25.796 & -4.3 & 1.049 \\
\hline$I R+M R$ & 11.809 & 12.805 & +8.4 & 12.582 & -1.8 & 1.053 \\
\hline$C R 1+I R+M R$ & 12.032 & 13.011 & +8.1 & 12.797 & -1.7 & 1.052 \\
\hline
\end{tabular}


TABLE 8.8 Control Rod Worths in ZPPR-17C

\begin{tabular}{|c|c|c|c|c|c|c|c|}
\hline Step $^{a}$ & $\begin{array}{c}\text { Control } \\
\text { Rodsa }\end{array}$ & $\begin{array}{l}\text { Experimental } \\
\text { Worth, } \$ \\
\end{array}$ & $\begin{array}{c}\text { Statistical } \\
10, \% \\
\end{array}$ & $\begin{array}{c}\text { Correlated } \\
10, \% \\
\end{array}$ & $\begin{array}{c}\text { Calculated } \\
k_{\mathrm{eff}} \\
\end{array}$ & $\begin{array}{l}\text { Calculated } \\
\text { Worth, } \$^{\mathrm{c}} \\
\end{array}$ & $C / E$ \\
\hline 7 & $6 \mathrm{IR}$ & 5.869 & 0.132 & 0.870 & 0.970615 & 5.651 & 0.963 \\
\hline 6 & $5 I R$ & 5.039 & 0.187 & 0.869 & 0.973270 & 4.814 & 0.955 \\
\hline 1 & $\begin{array}{l}3 \text { IR } \\
\text { symmetric }\end{array}$ & 3.390 & 0.111 & 0.868 & 0.978374 & 3.217 & 0.949 \\
\hline 5 & $\begin{array}{l}\text { 3IR } \\
\text { adjacent }\end{array}$ & 2.850 & 0.138 & 0.872 & 0.979829 & 2.765 & 0.970 \\
\hline 11 & $6 \mathrm{MR}$ & 7.682 & 0.114 & 0.862 & 0.965254 & 7.356 & 0.958 \\
\hline 10 & $5 M R$ & 6.045 & 0.190 & 0.868 & 0.970106 & 5.812 & 0.963 \\
\hline 9 & $\begin{array}{l}\text { 3MR } \\
\text { symmetric }\end{array}$ & 3.827 & 0.099 & 0.866 & 0.976877 & 3.684 & 0.963 \\
\hline 8 & $\begin{array}{l}\text { 3MR } \\
\text { adjacent }\end{array}$ & 3.052 & 0.123 & 0.873 & 0.979077 & 2.999 & 0.983 \\
\hline 3 & $3 I R+3 M R$ & 6.613 & 0.187 & 0.865 & 0.968448 & 6.338 & 0.958 \\
\hline 2 & $3 I R+2 M R$ & 5.370 & 0.168 & 0.868 & 0.972151 & 5.166 & 0.962 \\
\hline 4 & $2 I R+3 M R$ & 5.636 & 0.192 & 0.866 & 0.971377 & 5.411 & 0.960 \\
\hline
\end{tabular}

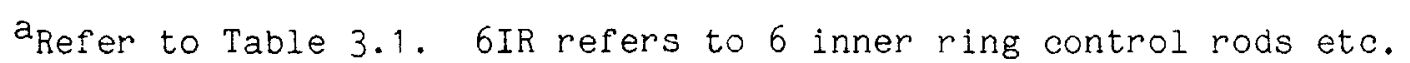

${ }^{b}$ Calculations 6 group xyz with half-z model, finite difference diffusion, reference $k=$ effective $=0.988823$.

$\mathrm{c}_{\text {Using Beffective }}=0.3357 \%$. 
TABLE 8.9 Control Rod Interaction Effects in ZPPR-17C

\begin{tabular}{lllll}
\hline & \multicolumn{4}{c}{ Worth Per Rod } \\
\cline { 2 - 5 } Rod Bank & Measured, $\$$ & $\sigma_{S}, \%$ & Calculated, $\$$ & C/E \\
\cline { 3 - 5 } & & & & \\
6IR & 0.978 & 0.13 & 0.942 & 0.963 \\
5IR & 1.008 & 0.19 & 0.963 & 0.955 \\
3IR adjacent & 0.950 & 0.14 & 0.922 & 0.970 \\
3IR symmetric & 1.130 & 0.11 & 1.072 & 0.949 \\
3IR symmetric with & 0.929 & 0.46 & 0.885 & 0.953 \\
3MR inserted & & & & \\
2IR with & 0.905 & 0.62 & 0.864 & 0.955 \\
3MR inserted & & & & \\
6MR & 1.280 & 0.11 & 1.226 & 0.958 \\
5MR & 1.209 & 0.19 & 1.162 & 0.962 \\
3MR adjacent & 1.017 & 0.12 & 1.000 & 0.983 \\
3MR symmetric & 1.276 & 0.10 & 1.228 & 0.963 \\
3MR symmetric with & 1.074 & 0.40 & 1.040 & 0.969 \\
3IR inserted & & & & \\
2MR with & 0.990 & 0.49 & 0.974 & 0.984 \\
3IR inserted & & & & \\
\hline
\end{tabular}


TABLE 8.10 Comparison of Control Rod Worths in ZPPR-17C using Different Calculation Models

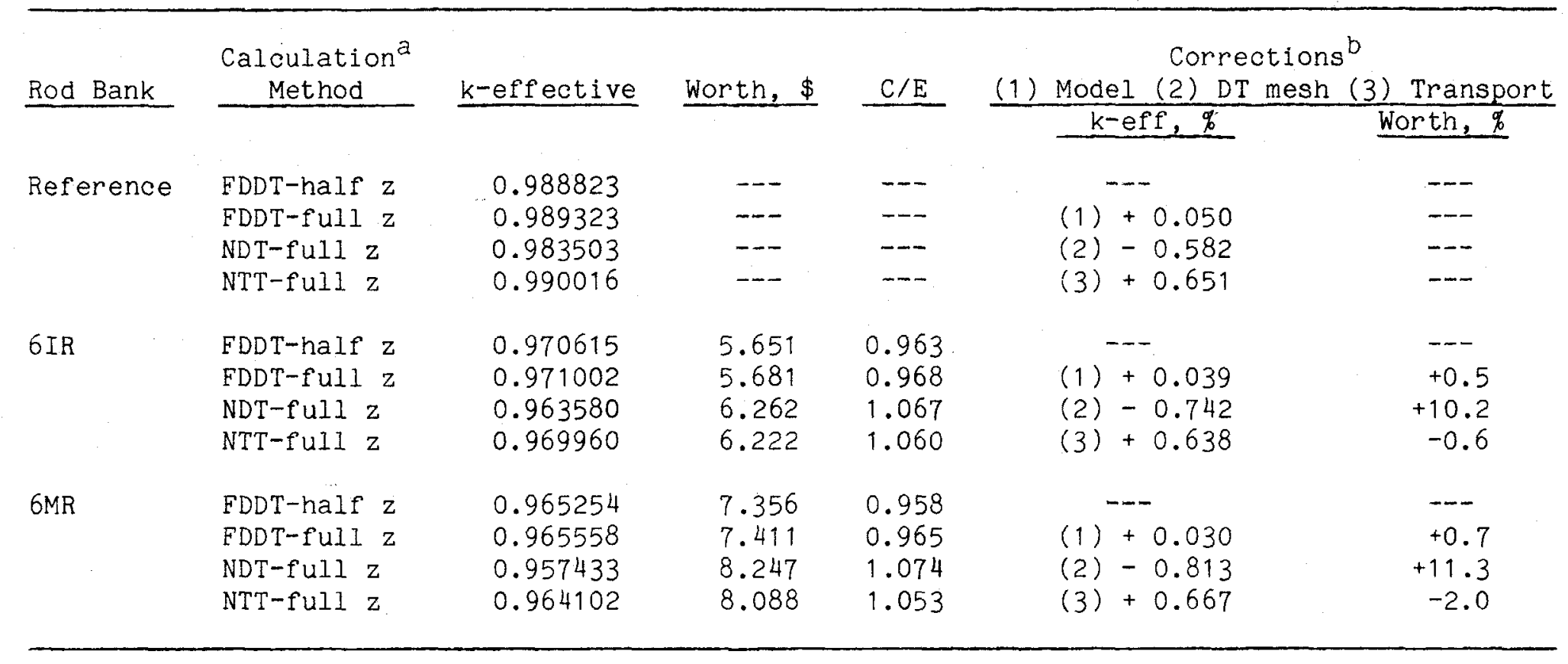

${ }^{a_{F D D T}}=$ finite difference diffusion, NDT = nodal diffusion, NTT = nodal transport.

$\mathrm{b}$ (1) Model correction half-z to full-z, (2) FDDT mesh $55 \mathrm{~mm}$ to effective fine mesh in NDT,

(3) fine-mesh diffusion (NDT) to transport (NTT). 
TABLE 8.11 Comparison of Control Rod Worths in ZPPR-17B and ZPPR-17C

\begin{tabular}{|c|c|c|c|c|c|}
\hline $\begin{array}{c}\text { Control } \\
\text { Rods } \\
\end{array}$ & Core & $\begin{array}{l}\text { Measured } \\
\text { Worth, \$ }\end{array}$ & $\begin{array}{c}\text { Total } \\
\text { Uncertainty, } \%\end{array}$ & $\begin{array}{l}\text { FDDT } \\
\mathrm{C} / \mathrm{E} \\
\end{array}$ & $\begin{array}{l}\text { NTT } \\
\text { C/E }\end{array}$ \\
\hline $6 I R$ & $\begin{array}{l}17 \mathrm{~B} \\
17 \mathrm{C}\end{array}$ & $\begin{array}{l}5.822 \\
5.869\end{array}$ & $\begin{array}{l}0.87 \\
0.88\end{array}$ & $\begin{array}{l}0.954 \\
0.968\end{array}$ & $\begin{array}{l}1.054 \\
1.060\end{array}$ \\
\hline $6 \mathrm{MR}$ & $\begin{array}{l}17 \mathrm{~B} \\
17 \mathrm{C}\end{array}$ & $\begin{array}{l}8.318 \\
7.682\end{array}$ & $\begin{array}{l}0.87 \\
0.87\end{array}$ & $\begin{array}{l}0.968 \\
0.965\end{array}$ & $\begin{array}{l}1.045 \\
1.053\end{array}$ \\
\hline $5 \mathrm{MR}$ & $\begin{array}{l}17 \mathrm{~B} \\
17 \mathrm{C}\end{array}$ & $\begin{array}{l}6.537 \\
6.045\end{array}$ & $\begin{array}{l}0.87 \\
0.89\end{array}$ & $\begin{array}{l}0.977 \\
0.969^{a}\end{array}$ & $\cdots$ \\
\hline
\end{tabular}

$\mathrm{a}_{\text {Assuming }}+0.7 \%$ modelling correction for 6MR (Table 8.10). 
TABLE 8.12 Calculations of Control Rod worths in ZPPR-17B Using 21 Group Data

\begin{tabular}{|c|c|c|c|c|}
\hline Case & & k-effective $^{a}$ & Worth $\$$ & $\begin{array}{c}\text { Group } \\
\text { Correction } \% \\
\end{array}$ \\
\hline Reference & $\begin{array}{r}6 G \\
21 G\end{array}$ & $\begin{array}{l}0.982957 \\
0.983351\end{array}$ & - & $(+0.00039 \Delta k)$ \\
\hline CR1 & $\begin{array}{r}6 \mathrm{G} \\
21 \mathrm{G}\end{array}$ & $\begin{array}{l}0.979565 \\
0.980200\end{array}$ & $\begin{array}{l}1.044 \\
0.969\end{array}$ & -7.4 \\
\hline $6 I R$ & $\begin{array}{r}6 G \\
21 G\end{array}$ & $\begin{array}{l}0.963118 \\
0.964591\end{array}$ & $\begin{array}{l}6.211 \\
5.862\end{array}$ & -5.9 \\
\hline $6 \mathrm{MR}$ & $\begin{array}{r}6 G \\
21 G\end{array}$ & $\begin{array}{l}0.954720 \\
0.956123\end{array}$ & $\begin{array}{l}8.918 \\
8.583\end{array}$ & -4.0 \\
\hline $120 \mathrm{R}$ & $\begin{array}{r}6 \mathrm{G} \\
21 \mathrm{G}\end{array}$ & $\begin{array}{l}0.908321 \\
0.911074\end{array}$ & $\begin{array}{l}24.776 \\
23.911\end{array}$ & -3.7 \\
\hline
\end{tabular}

$a_{\text {Nodal diffusion calculations }}$ 
TABLE 8.13 Calculations of Control Rod Worths in ZPPR-17B with the TRITAC Code

\begin{tabular}{llcc} 
Case & k-effective $^{a}$ & $\begin{array}{c}\text { Control Rod } \\
\text { Worth, } \$\end{array}$ & $\begin{array}{c}\text { Streaming } \\
\text { Correction, } \$\end{array}$ \\
$\begin{array}{llll}\text { Subcritical Reference } \\
\text { CR1 }\end{array}$ & 0.989229 & - & $(-0.00236 \Delta \mathrm{k})$ \\
6 Inner Ring & 0.985801 & 1.010 & -0.018 \\
6 Middle Ring & 0.969377 & 5.927 & +0.026 \\
12 Outer Ring & 0.961333 & 8.694 & +0.006 \\
\hline
\end{tabular}

${ }^{a}$ Calculations 6 group, xyz, S4, $5 \mathrm{~cm}$. mesh size with average compositions for each cell-type.

${ }^{b}$ Not included in TRITAC results. 
TABLE 8.14 Calculations of Control Rod Banks in ZPPR-17B by Different Methods

\begin{tabular}{|c|c|c|c|c|c|}
\hline Rods & $\begin{array}{l}\text { Applied } \\
\text { Group } \\
\text { Correction, \% } \\
\end{array}$ & $\begin{array}{c}\text { F.D. } \\
\text { Diffusion } \\
C / E^{a} \\
\end{array}$ & $\begin{array}{c}\text { Nodal } \\
\text { Diffusion } \\
\mathrm{C} / \mathrm{E} \\
\end{array}$ & $\begin{array}{c}\text { Nodal } \\
\text { Transport } \\
\mathrm{C} / \mathrm{E} \\
\end{array}$ & $\begin{array}{c}\text { TRITAC } \\
\quad \mathrm{C} / \mathrm{E} \\
\end{array}$ \\
\hline CR1 & -7.2 & 0.885 & 0.968 & 0.966 & 0.920 \\
\hline 6 Inner Ring & -5.6 & 0.925 & 1.007 & 0.995 & 0.965 \\
\hline 6 Middle Ring & -3.8 & 0.931 & 1.031 & 1.005 & 0.981 \\
\hline 12 Outer Ring & -3.5 & 0.953 & 1.064 & 1.015 & 1.009 \\
\hline
\end{tabular}

Finite difference, $50 \mathrm{~mm}$ mesh size. 


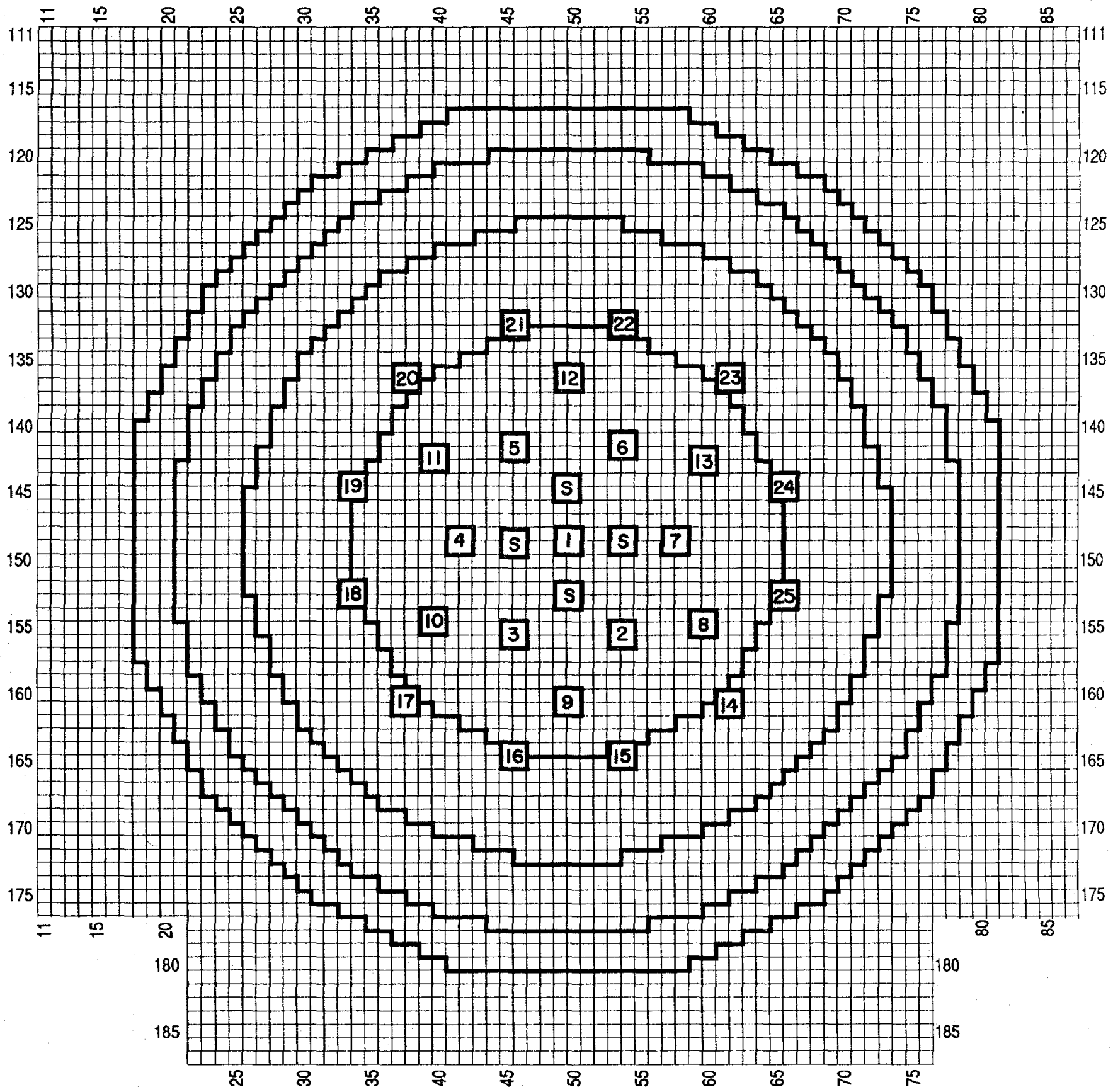

n CRP location

S Special measurements of four control rods and CRP's

Fig. 8.1 Control Positions used in ZPPR-17A Measurements. 
148

149

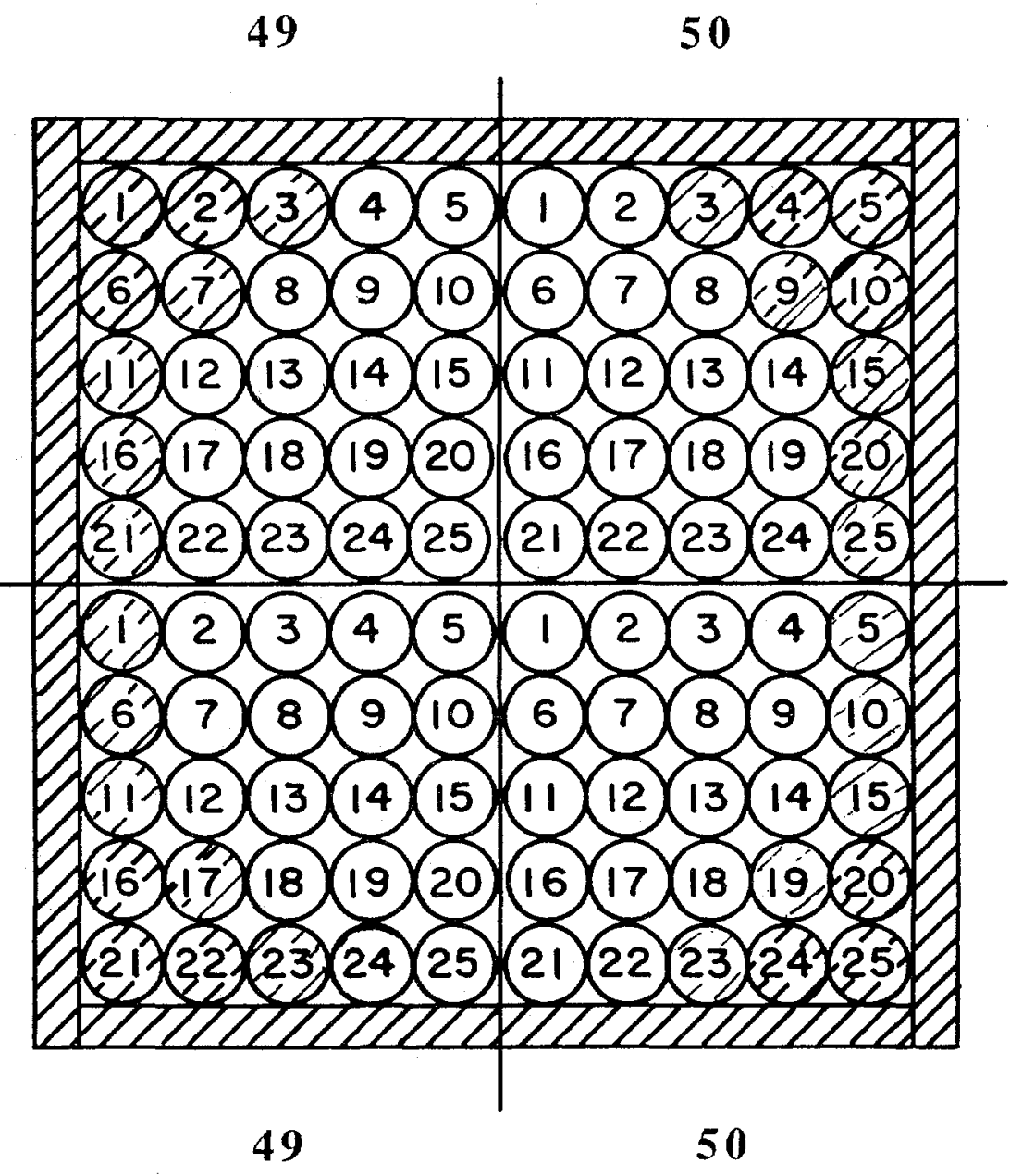

\section{8}
$\#$
$B_{4} C$ PELLETS
\# Stainless steel pins
$\bigoplus$ stainless steel plates

Fig. 8.2 Loading Pattern for Close-packed Pin Control Rod. 


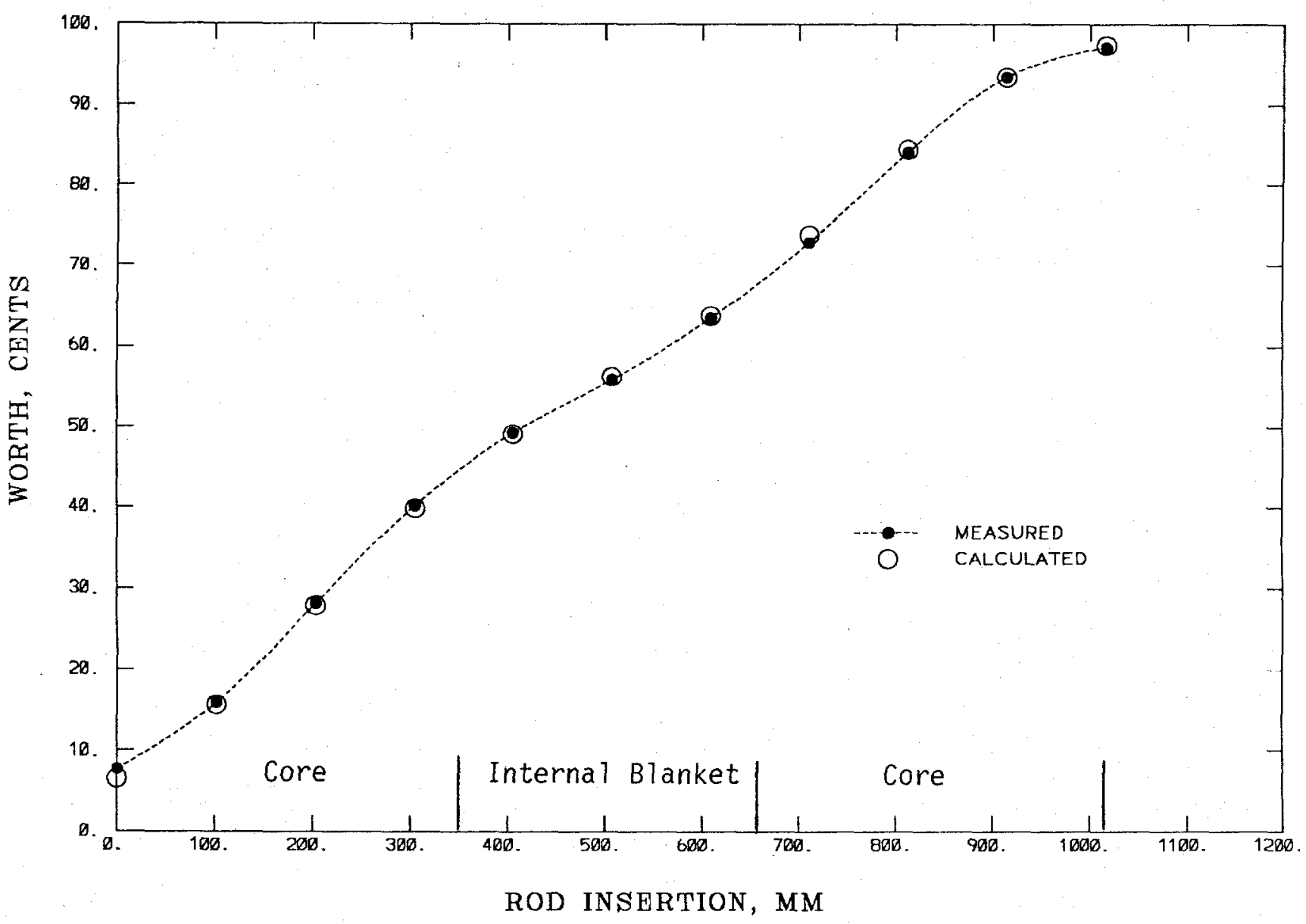

Fig. 8.3 Worth Profile for Mockup Central Control Rod in ZPPR-17B. 


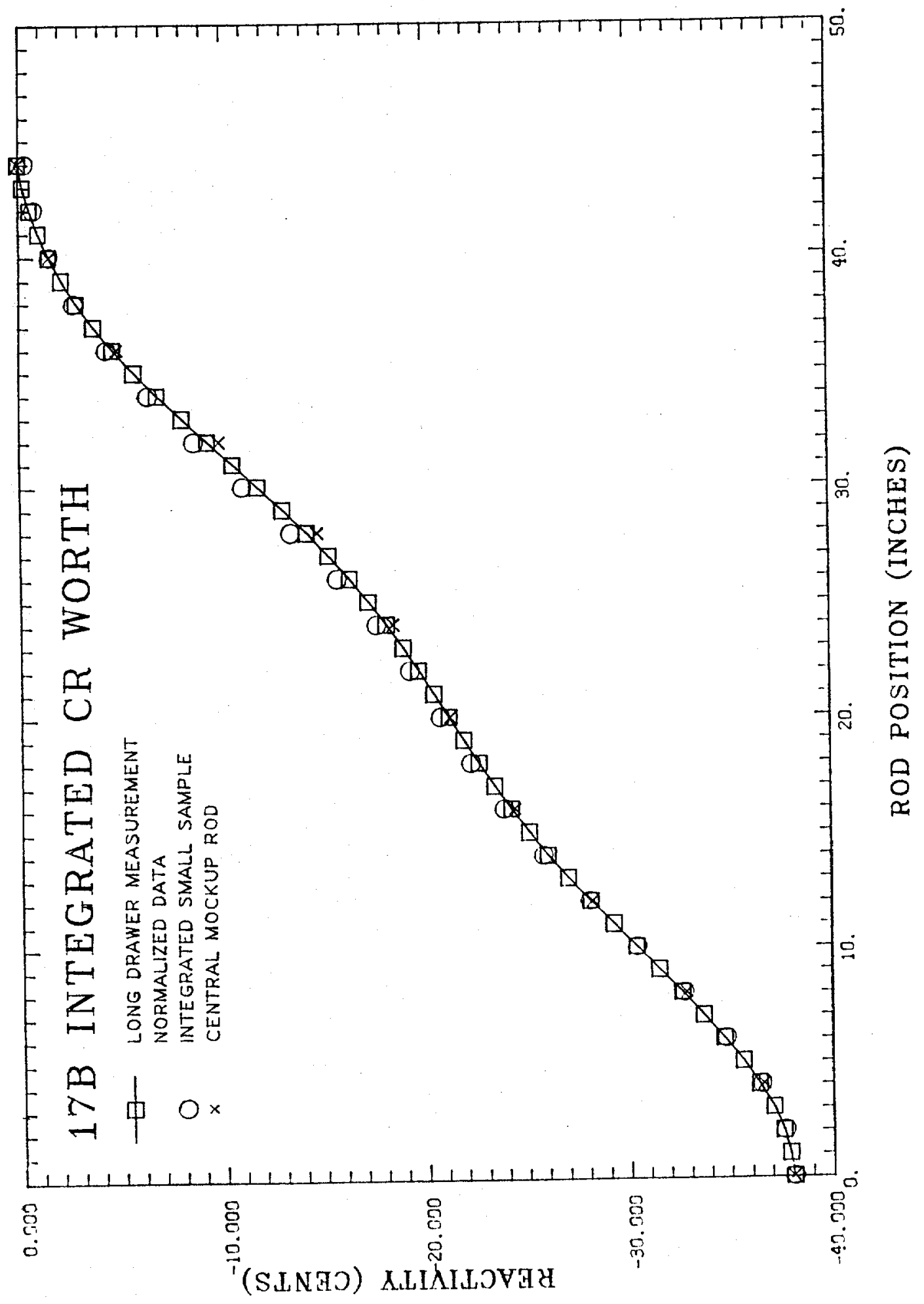




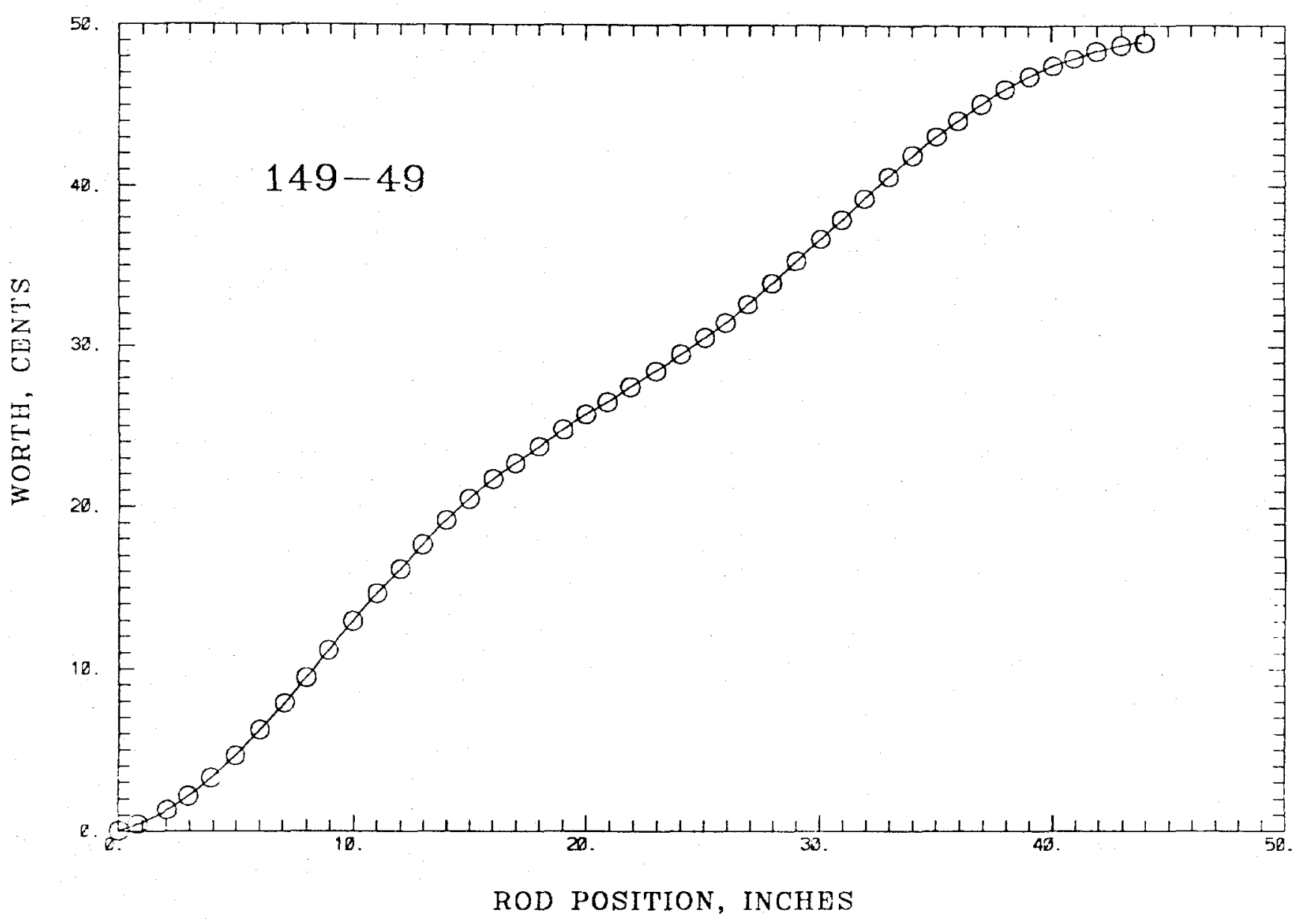

Fig. 8.5 Measured Axial Worth Profile for $\mathrm{B}_{4} \mathrm{C}$ in $149-49 / 249-49$ in $\mathrm{ZPPR}-17 \mathrm{C}$. 


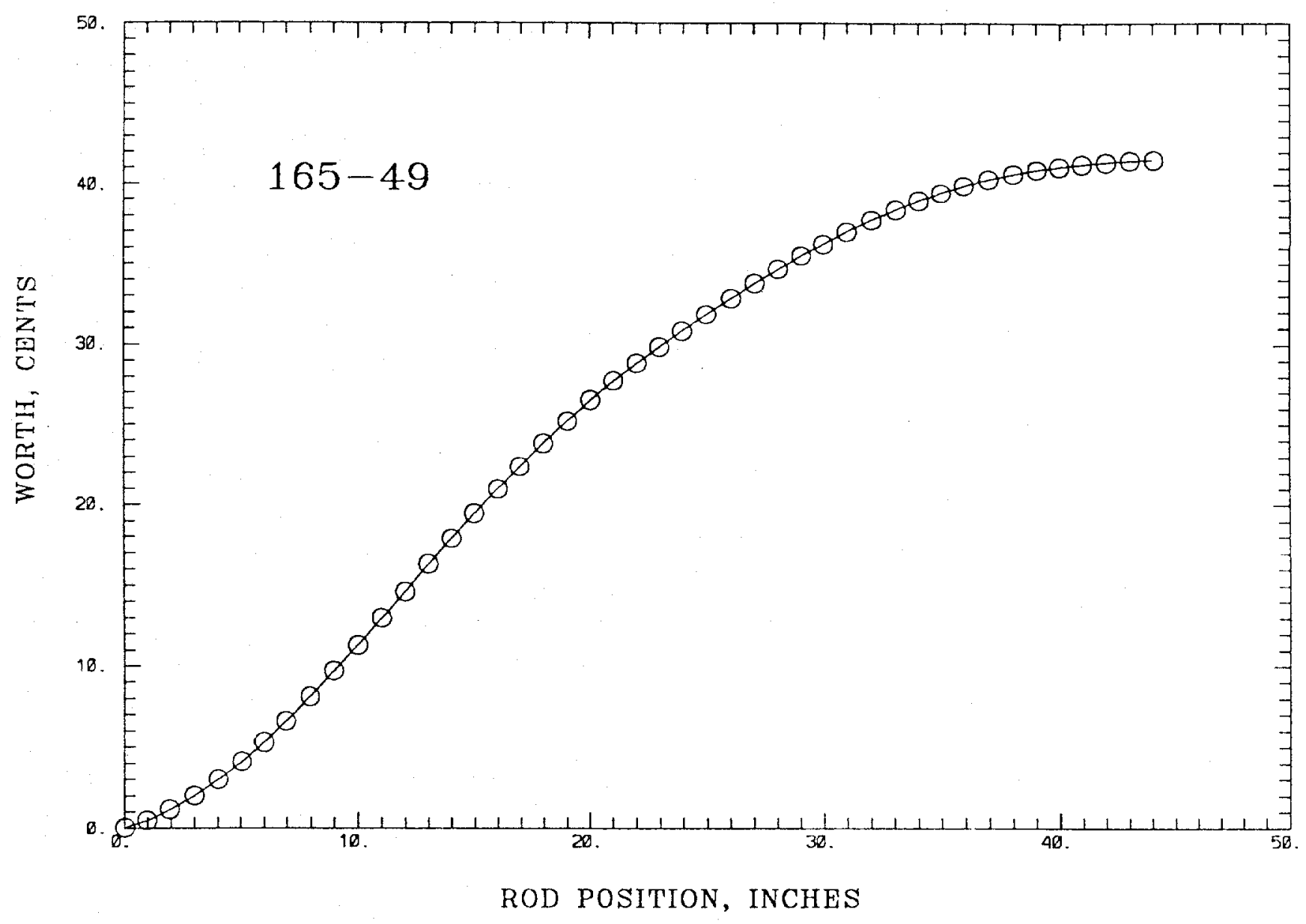

Fig. 8.6 Measured Axial Worth Profile for $\mathrm{B}_{4} \mathrm{C}$ in $165-49 / 265-49$ in ZPPR-17C. 


\section{SODIUM VOID WORTHS}

Sodium void worths were measured only in ZPPR-17A. Two different techniques were used. In one technique, a subcritical reactivity difference was measured after substituting empty cans for sodium-filled cans in two different zones of the assembly. Void-can substitution was done in a series of axial steps. The second technique used the new plate column oscillator devices described in section 2 to move a single column of sodium or void cans axially through one assembly half. Oscillator measurements were made at ten different radii.

\subsection{Plate Substitution Results}

The reference configuration for the plate substitution measurements was the ZPPR-17A subcritical reference. Two zones were voided. The first zone was just outside the region with the internal blanket and contained 36 drawers per assembly half. The second zone was at the core center and contained 52 drawers per assembly half. The zones are shown in Fig. 9.1. The sodium carbonate in double-fuel-column drawers was not included in the voiding. Reactivity changes were measured using the modified source multiplication method. Both zones were voided in a series of cumulative axial steps which corresponded to the lengths of the sodium cans. In the outer zone, the steps were $\pm 203 \mathrm{~mm}, \pm 406 \mathrm{~mm}, \pm 508 \mathrm{~mm}$ and $\pm 787 \mathrm{~mm}$. In the inner core, the fuel region was voided first $( \pm 152 \mathrm{~mm}$ to $\pm 330 \mathrm{~mm}, \pm 152 \mathrm{~mm}$ to $\pm 508 \mathrm{~mm}$ ) then the internal blanket (0 to $152 \mathrm{~mm}$ ) and finally the external axial blanket to $\pm 787 \mathrm{~mm}$. Step reactivity worths are given in Table 9.1. Further details of the measurements were given in reference 1 . The uncertainties are dominated by a 0.2 cent contribution from reproducibility of the reactivity of the subcritical reference before and after the voiding. Other, smaller, components from reactivity scale calibration $(0.7 \%)$ and statistical uncertainties (0.04ф) are included. In the measurements, the steel mass changed slightly with each step due to mass differences between sodium filled cans and empty cans. Reactivity effects of these steel mass changes were estimated by calculations to be smal1; $0.06 \phi$ for the $0.34 \mathrm{~kg}$ case in the inner core. Because the steel corrections 
are small compared to the uncertainties, no reactivity corrections were made to the step worths in Table 9.1 .

Calculations were made for the central zone voiding. Calculated results were obtained by diffusion theory in $\mathrm{rz}$ geometry. Exact perturbation theory (voided adjoint) was used to show the non-leakage and leakage components for each step. Values of $C-E$ are given for the various steps in Table 9.2. Calculated values are within 7 to 15\% of experiment for the core and internal blanket voiding. However, the present calculated values are lower than the measured values in the steps of small leakage fraction, in contrast to other results with ENDF/B-V.2 data where calculation is a few percent higher. It is concluded that further studies of cross section processing for void cells in different environments and transport calculations will be necessary to refine these results. Analysis of the outer-core measurements is not complete.

\subsection{Oscillator Results}

Sodium worth oscillator measurements were made in ten locations which are shown in Fig. 9.1. Five locations (148-49, 148-43, 147-39, 14835, 148-34) contained single-fuel-column drawers with internal blanket, three (147-33, 148-32, 147-27) contained single-fuel-column drawers with no internal blanket and two (148-25 and 148-23) contained radial blanket drawers.

Reactivity was derived as a function of column position by inverse kinetics analysis of power history recorded by ex-core $\mathrm{BF}_{3}$ chambers. Three full oscillation cycles were used. Two measurements were made in each location; one with sodium-filled cans and one with empty cans and the sodium effect obtained by difference. The mass of sodium in one column was about $358 \mathrm{gm}$ and the mass of steel about $264 \mathrm{gm}$.

Results for six representative locations are given in Fig. 9.2. Values in the figure are integrated reactivities for the front of the sodium column at various distances from the front of the drawer. Zero position corresponds to the sodium column against the front of the drawer. The zero 
of reactivity is somewhat arbitrarily set to correspond to the fully withdrawn sodium column. The shapes of the curves are qualitatively similar for all core locations except 147-27. In these three core locations, the sodium worth is small and positive in the axial blanket, reaches a maximum positive value between $254 \mathrm{~mm}$ and $508 \mathrm{~mm}$ from the interface and is negative near the interface. At 147-27, the outermost portion of the core, the worth is essentially always positive and increases monotonically toward the interface. The innermost blanket result, 148-25, is similar to the outer core result. Deeper into the blanket, in 148-23, the sodium worth is very small with only a small axial variation.

Statistical uncertainty of the oscillator results was about $0.0015 \$$ at $25 \mathrm{~mm}$ displacement intervals, or about $2 \%$ of the maximum reactivity values. Detailed results for the oscillator measurements were given in reference 1 .

of perhaps greater interest in axially heterogeneous cores, is the differential sodium void worth. Figure 9.3 shows both calculated and measured differential worth results for the core center (148-49). The measured values are averages for three oscillations to reduce statistical fluctuations on the differences between successive steps. The void reactivities are commonly analyzed in terms of the leakage component, which varies with position approximately as the square of the flux gradient, and the non-leakage component which varies approximately as the square of the total flux. These two calculated components (by first-order perturbation) and their sum are also shown in Fig. 9.3. The leakage component has two minima, produced by inward leakage to the internal blanket and outward leakage to the axial blanket with a zero component below the axial center of the fuel. The calculated total worth reproduces the measured shape quite we11. 
TABLE 9.1 Step Reactivity Worths for Sodium Voiding in ZPPR-17A

\begin{tabular}{|c|c|c|c|c|c|}
\hline Step ${ }^{a}$ & $\begin{array}{l}\text { Mass Sodium } \\
\text { Voided, } \mathrm{kg} \\
\end{array}$ & $\begin{array}{l}\text { Mass Steel } \\
\text { Added, } \mathrm{kg}\end{array}$ & $\begin{array}{l}\text { Measured } \\
\text { Reactivity } \\
\text { Change, } \phi \\
\end{array}$ & $\begin{array}{c}\text { Total } \\
\text { Uncertainty } \\
10, Q \\
\end{array}$ & $\begin{array}{r}\text { Specific } \\
\text { Reactivity } \\
\phi / \mathrm{kg}(\mathrm{Na}) \\
\end{array}$ \\
\hline \multicolumn{6}{|l|}{ Outer Core } \\
\hline $\begin{array}{r}0-203 \mathrm{~mm} \\
203-406 \mathrm{~mm} \\
406-508 \mathrm{~mm} \\
508-787 \mathrm{~mm}\end{array}$ & $\begin{array}{r}12.625 \\
12.624 \\
6.160 \\
21.097\end{array}$ & $\begin{array}{l}0.111 \\
0.111 \\
0.065 \\
0.071\end{array}$ & $\begin{array}{l}+6.82 \\
+1.68 \\
-2.09 \\
-3.87\end{array}$ & $\begin{array}{l}0.21 \\
0.30 \\
0.30 \\
0.30\end{array}$ & $\begin{array}{l}+0.540 \\
+0.133 \\
-0.339 \\
-0.183\end{array}$ \\
\hline \multicolumn{6}{|l|}{ Inner Core } \\
\hline $\begin{array}{r}152-330 \mathrm{~mm} \\
330-508 \mathrm{~mm} \\
0-152 \mathrm{~mm} \\
508-787 \mathrm{~mm}\end{array}$ & $\begin{array}{l}15.472 \\
15.472 \\
16.821 \\
30.741\end{array}$ & $\begin{array}{l}0.341 \\
0.340 \\
0.020 \\
0.101\end{array}$ & $\begin{array}{l}+4.92 \\
+1.35 \\
+7.98 \\
-4.52\end{array}$ & $\begin{array}{l}0.21 \\
0.30 \\
0.31 \\
0.30\end{array}$ & $\begin{array}{l}+0.318 \\
+0.087 \\
+0.474 \\
-0.147\end{array}$ \\
\hline
\end{tabular}

${ }^{a}$ Voided symmetrically in each half. 


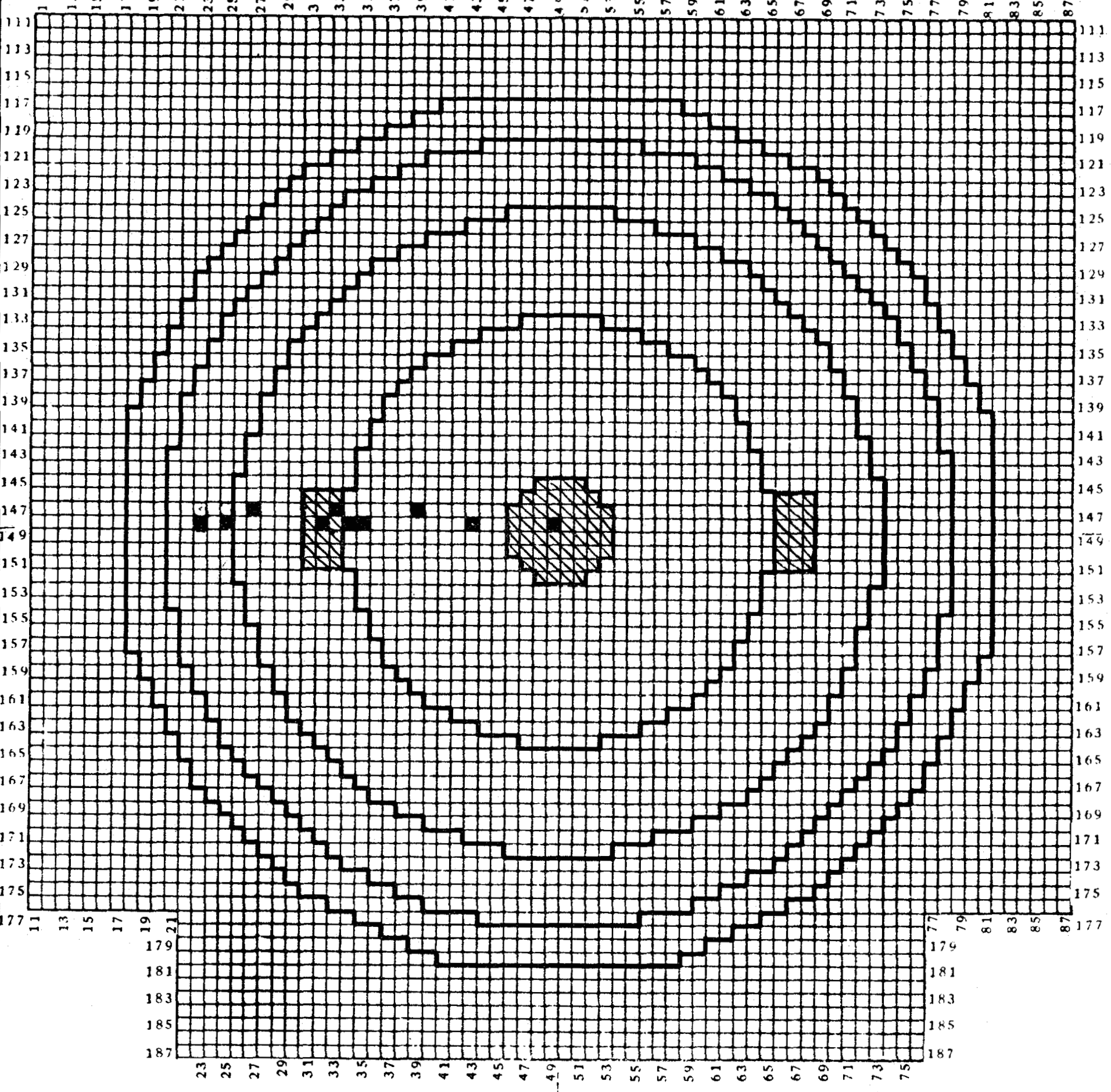

$\checkmark$ Void Zones

- Oscillator Measurement

Fig. 9.1 Locations of Sodium Void Worth Measurements in ZPPR-17A. 


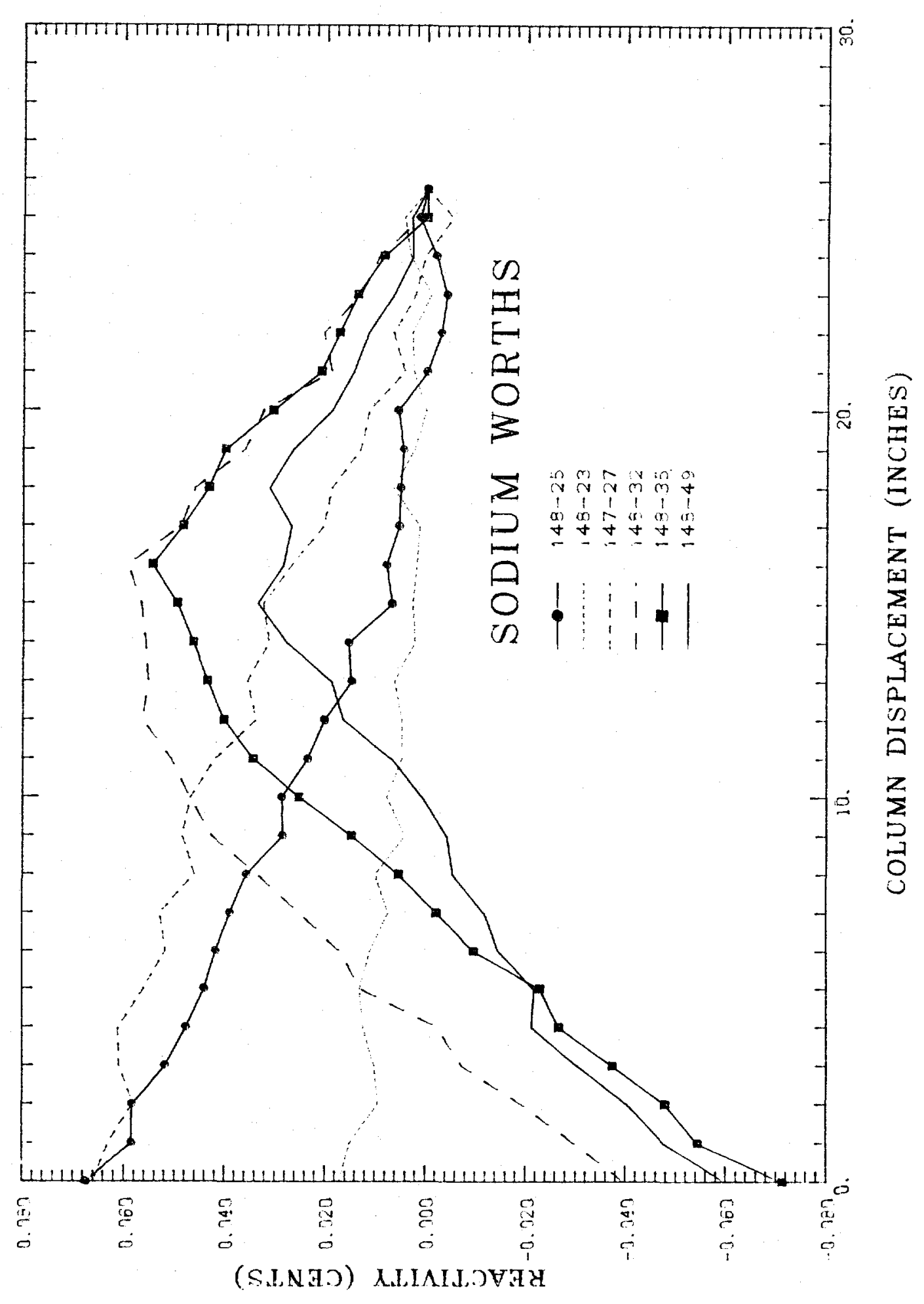

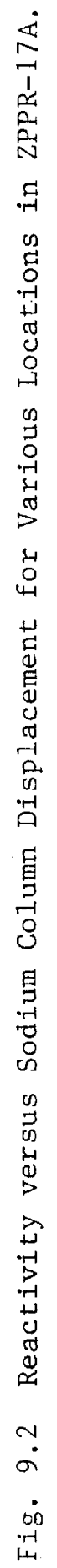




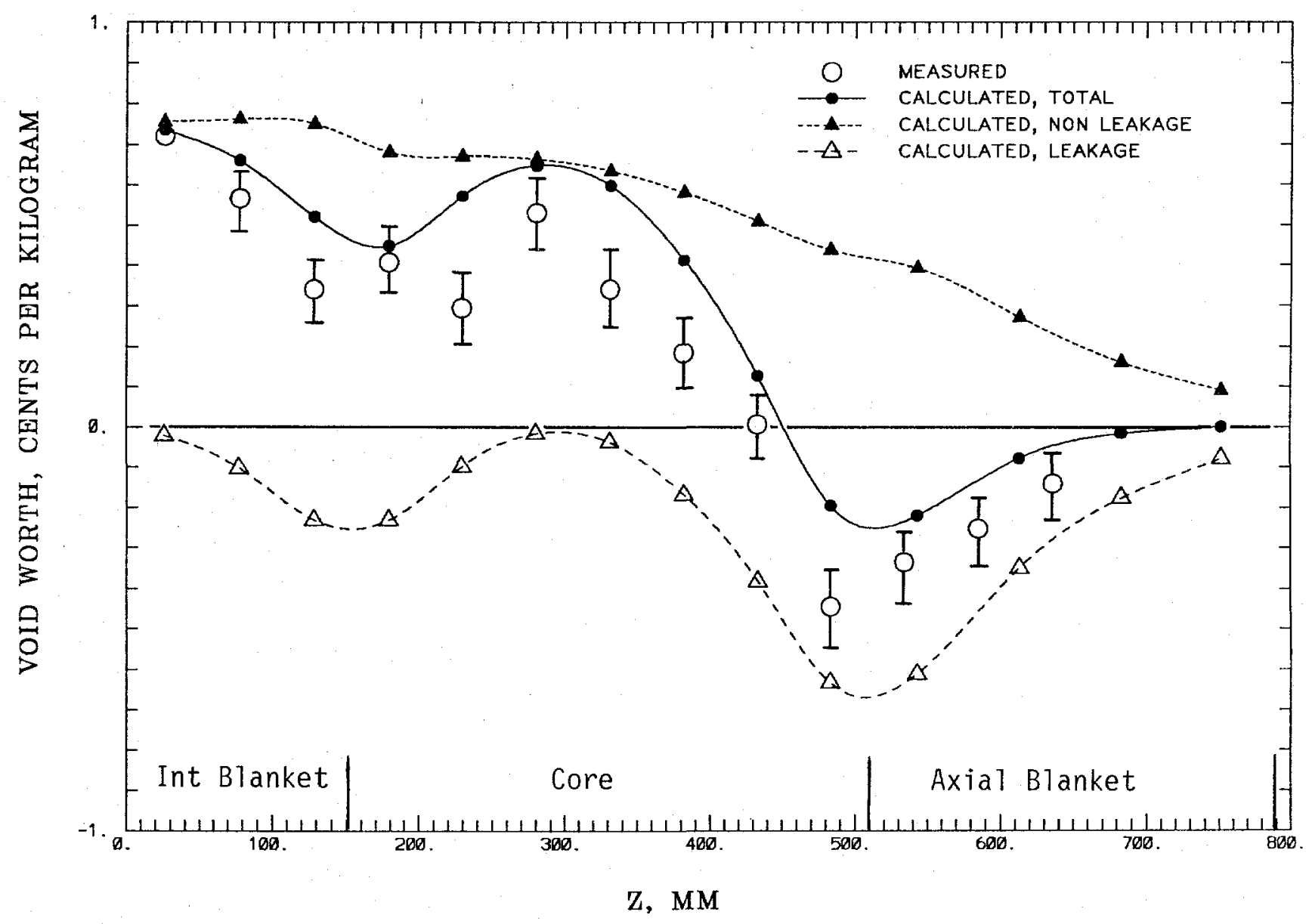

Fig. 9.3 Calculated and Measured Sodium Void Worths as a Function of Axial Position in ZPPR-17A. 
10. CALCULATIONS OF BOWING, EXPANSION AND SMALL-SAMPLE WORTH EXPERIMENTS IN

10.1. Introduction

The purpose of these experiments was to test the accuracy of reactivity feedback calculations in reactor safety analyses. The inherent safety phenomena addressed by the experiments are bowing, radial expansion, axial expansion and sodium density change. Reactivity feedbacks related to core disruption were addressed by measuring the worth of fuel, structure, control and coolant samples at various locations.

The measurements are described thoroughly in section 10 of Ref. 3. There was one static experiment (plate shifting) and a large number of material oscillation experiments. Table 10.1 and Figure 10.1 give an overview of the oscillator experiments that were done.

The methods used to calculate these experiments generally are consistent with methods used by reactor designers. In some cases, however, extra calculational effort was required to account for phenomena that are more important in the critical experiments.

XYZ geometry and nodal diffusion theory were used in calculating each of the experiments. Models of the exact reactor loadings were used to analyze the plate shifting experiment. For all other experiments a model of the critical reference configuration with all operational control rods fully withdrawn was used. Except for the radial tube oscillator (RTO) experiments, the true reactor configurations for the oscillator experiments were essentially the same as the critical reference and the control rods were withdrawn into the axial blanket. Use of the reference configuration for the RTO calculations is justified by the fact that the configuration differences affected the fission rate near the oscillator by less than $1 \%$, acoording to the 64 fission chamber data.

The 21 group cross sections based on ENDF/B Version 5.2 data and produced by the buckling recycle method were always used (see section 4 ). 
Calculated reactivities were converted from $\Delta k / k$ to cents using a beta-effective value of 0.003409 . This value is based on ENDF/B Version 5 delayed neutron data. It includes an adjoint correction factor of 1.0087 that accounts for the error in the standard adjoint flux caused by using forward-flux weighting in the cross section homogenization ${ }^{17}$.

In each of the remaining subsections a class of experiments is discussed. The analysis techniques are described, representative $\mathrm{C} / \mathrm{E}$ results are presented and some observations about the results are made. In all cases the quoted uncertainties for the C/Es include only experimental sources, not calculated ones. C/Es are not displayed when the uncertainty exceeds $20 \%$. A complete description of the calculations and C/Es is given in Ref. 18 and many of them are included in Ref. 19. The C/Es quoted in Ref. 19 for the ZPPR-17 axial expansion oscillator and sodium plate column oscillator experiments are $1.7 \%$ larger than those appearing here because an error was found after Ref. 19 was submitted.

\subsection{RTO and ATO Small-Sample Worth Experiments}

Worths of 4 or 5 sample materials were measured using both the radial tube oscillator (RTO) and axial tube oscillator (ATO) techniques. The locations of the radial traverse and the 3 axial traverses - ATO-1, ATO-2 and ATO-3 - are indicated in Fig. 10.1.

The basic method for computing the sample worths was first-order perturbation theory (FOP) within a nodal diffusion theory framework. These nodal perturbation calculations used the fission emission spectrum of the sample material when it was fissionable. They yielded the node-average worths of infinitesimal, cell-spanning samples. A computer program interpolated the node-average worths and applied a number of correction factors, yielding the worths of the true finite samples at their exact reactor locations.

In most cases "detector" cross sections were used to compute the worths. These cross sections are homogeneously self-shielded using the cell-average composition. For PU-30 outside the core and for DU-6 
everywhere detector cross sections provide an inadequate resonance shielding representation. For DU 6 the ${ }^{238} \mathrm{U}$ cross sections of a ZPPR fuel plate (in a double-fuel-column drawer) was used everywhere because the mean chord length of ${ }^{238} \mathrm{U}$ in the fuel plate is only $5 \%$ different (smaller) than in DU 6 . For PU-30 in locations outside the core we generated cross sections that have the resonance shielding and broad group effects of the finite sample size built into them.

Correction factors to account for self multiplication and broad group-type self shielding in the samples were computed with the SARCASM code $^{20}$.

Adjoint heterogeneity corrections were computed for the $\Delta k / k$ values of sample worths in addition to the adjoint correction of beta-effective. The prescription of Ref. 17 was followed.

For the ATO traverses, corrections were computed to get from cell-spanning worth to worth at the sample location within the cell. These correction factors were obtained using broad group, transport perturbation theory FOP worth calculations. No in-cell position corrections were computed for the RTO experiments because there the samples always spanned the unit cells.

Global transport corrections were computed for all the worth traverses. These correction factors were obtained by comparing sample worths from fine mesh, $S_{4}$ transport theory and fine mesh finite-difference diffusion theory calculations.

Global axial position corrections were computed for the RTO traverses. These corrections are essentially factors that interpolate to. the tube location within the first axial node of the XYZ ZPPR-17A model. This is necessary because the axial node spacing is large (spacings in $X$ and $Y$ are not) and the traverse processing code interpolated only along the direction of traverse.

The experimental, calculated and C/E results for some of the RTO 
and ATO worth traverses that included the internal blanket are shown in Tables 10.2 through 10.6 and Figures 10.2 through 10.6. For the radial traverses the position 非 is the matrix column, and for the axial traverses it is simply the stop-point number starting from the innermost position. The position $X$ or $Z$ is the distance from the center of the assembly to the center of the sample. The initial $\mathrm{C} / \mathrm{E}$ is the result without correction factors; it uses the interpolated nodal FOP calculated value and includes only special cross section effects (for PU-30 outside the core, and for DU-6). The size factor is from the SARCASM code. The position factor is the global axial factor for the radial traverses and is the in-cell factor for the axial traverses.

Self shielding and other sample size effects for PU-30 outside the core have not been delt with very successfully. A symptom of these size effect problems is the C/E discontinuity that occurs at every core/blanket interface in all the PU-30 traverses. Two alternative treatments for PU-30 outside the core were tried but results from them were also poor.

The radial traverses all have C/Es that are less than unity in the internal blanket, and for all of these traverses the C/Es increase substantially with increasing radius through the internal blanket and core. This trend with radius has been observed in $2 P P R-17 \mathrm{~A}$ radial reaction rate traverses and in similar experiments in other large LMR critical assemblies. However, the $\mathrm{C} / \mathrm{E}$ increase for the $Z \mathrm{PPR}-17 \mathrm{~A}$ radial worth traverses is larger than seen previously. The C/Es from the axial traverses generally look better than those from the radial traverses. The $\mathrm{C} / \mathrm{Es}$ are within a few percent of unity and nearly constant axially in most cases.

There seems to be some inconsistency between the radial and axial traverses. The two kinds of traverses never sampled the same reactor location but, assuming radial symmetry, they did sample similar locations. The difference between RTO and ATO C/Es from the similar locations are shown In Table 10.7. In some cases there is no discrepancy but C/E differences as large as $8 \%$ can be seen. The cause of this has not been identified. 
and axial traverse results. They are shown in Table 10.8. Only values with uncertainties of $10 \%$ or less have been used to obtain the averages. In the core region, most of the C/Es are within $5 \%$ of unity and all are within about 10\%. The $\mathrm{C} / \mathrm{E}$ discrepancies generally are larger in the blankets, but most $\mathrm{C} /$ Es are within $12 \%$ of unity. The problem of increasing C/E with increasing core radius is evident from comparing the core region ATO C/ES for $\mathrm{B}-1$ and for $\mathrm{U}-6$, but the trend is not clear from the PU-30 ATO traverses.

\subsection{Neutron Importance Traverse}

The radial profile of the neutron importance was inferred from ${ }^{252} \mathrm{Cf}$ traverses with the RTO. The ${ }^{252} \mathrm{Cf}$ neutron source importance was calculated consistently with the RTO small-sample worth calculations.

The experimental and calculated results are shown in Table 10.9 and Fig. 10.7. The experimental importance at the full-out position (not shown) was taken to be identically zero, and the uncertainty at that position was added in quadrature to the uncertainties at each of the other positions. The experimental importance was normalized to have a peak value of unity. The transport correction was normalized such that its average value in the core is unity. Then the corrected calculated values were normalized such that the average $\mathrm{C} / \mathrm{E}$ in the core is unity.

It can be seen that the error in the calculated importance has the same trend as for small-sample worths from the RTO; the C/E increases substantially with increasing radius through the internal blanket and core. Both of the correction factors aggravate this problem, but it is qualitatively the same with or without the corrections. One might expect that the error in the adjoint flux profile would be the same as the error in the forward flux profile, but that does not seem to be the case. The radial reaction rate traverses, which depend on the forward flux but not the adjoint, have $\mathrm{C} / \mathrm{E}$ tilts that are much smaller than the importance C/E tilt. Furthermore the magnitudes of the tilts in the PU-30 and B-1 RTO C/Es, which depend on both the forward and adjoint fluxes, are about the same as the magnitude of the importance $\mathrm{C} / \mathrm{E}$ tilt. Thus it appears that the sample worth 
C/E tilts are due more to an adjoint profile error than to a forward flux profile error.

\subsection{Plate Column Oscillator Experiments}

Plate column oscillators were used to measure the worth of removing $\mathrm{Pu}-\mathrm{U}$ fuel, $\mathrm{U}_{3} \mathrm{O}_{8}, \mathrm{Fe}_{2} \mathrm{O}_{3}$, sodium and void can (stainless steel) columns. The experiments are referred to as PCO-1 through PCO-10. All the measurements except PCO-5 were made at SFC matrix positions.

The moving plate column sometimes consisted of a mix of materials. In PCO-1 and PCO-3 it was stainless steel-clad Pu-U (standard ZPPR fuel) for the first $50.80 \mathrm{~cm}$ and stainless steel plates from there to the end of the column $(66.04 \mathrm{~cm})$. According to calculations the clad and steel plate contributions are each about 2\% of the column worth and are of opposite sign, so the net steel contribution is less than $1 \%$. In PCO-5 and PCO-6 the entire moving column was clad Pu-U; the presence of the clad reduces the calculated worth by about $2 \%$. The experimental worth of the void can column ( $\mathrm{PCO}-9$ ) was subtracted from the experimental worth of the clad sodium column (PCO-8) to get the net worth of sodium (PCO-8-9), which is the quantity for which calculations are presented.

Calculations of these experiments were done using first order perturbation theory, even though the perturbations are not believed to be first order. The perturbations were too small to cause significant global flux changes (with the possible exceptions of $\mathrm{PCO}-1,3$ and 6 ), but the disruption of the intracell flux was probably significant. No corrections were applied other than the adjoint correction to beta-effective. Accordingly the calculated results presented here are considered preliminary.

Actually an attempt to compute accurate worths was made in the case of sodium (PCO-8-9). Special attention was given to this experiment because it is the only one in the ZPPR-17 program that attempted to measure a coolant density feedback coefficient. To account for the disruptions to the standard SFC cell, cell average cross sections were computed for the PCO-8 and PCO-9 drawer loadings (with the moving columns inserted). Both of 
these cross section calculations used group- and region-dependent bucklings from the reference eigenvalue solution to account for the fact that the experiment was surrounded by a normal core. Comparing the sodium worths based on the standard and special cross sections yielded cell correction factors, which range from 0.94 to 0.96 . In addition, an adjoint correction factor for the $\Delta k / k$ values was computed, as described in Section 10.2 ; this factor is 0.83 , an unusually large adjustment.

The basic FOP calculations were done in same way as those for the radial and axial tube experiments. Nodal perturbation theory and the integral interpolation scheme were used. Core cell average cross sections were used to compute the worths because, of the standard types available, they probably best represent the plate columns in the unit cell.

Worth traverse results for the two traverses that included the internal blanket are shown in Tables 10.10 and 10.11 . The cumulative worths are for withdrawing the plate column the distance indicated from the full-in position. The incremental worths are for the change from the previous withdrawal position to the current one. There is a definite pattern of increasing $\mathrm{C} / \mathrm{E}$ with increasing withdrawal from the internal blanket, and $\mathrm{C} / \mathrm{Es}$ are higher in the core than in the internal blanket. This is similar to the RTO C/E behavior, but not like the ATO-1 PU-30 C/E behavior.

The experimental uncertainties used here are larger than those tabulated in Section 10 of Ref. 3. The increase is due to incorporating the estimated uncertainty in the withdrawal position. It is estimated that the withdrawal from full in is uncertain by about $0.04 \mathrm{~cm}$, but the distance between other positions is known to about $0.004 \mathrm{~cm}$. This has a significant effect only on small. withdrawals; for example this uncertainty is $11 \%$ for $0.38 \mathrm{~cm}$ withdrawal but less than $1 \%$ for $5 \mathrm{~cm}$ withdrawal.

A comparison between PCO experiment C/Es and radial and axial tube experiment $\mathrm{C} / \mathrm{ES}$ is shown in Table 10.12 . There is only a rough correspondence between the PCO and tube experiments; the sample compositions generally were quite different, the cell types where the measurements were done were often different, and the measurement positions were only roughly 
comparable. In addition, the preliminary nature of the PCO calculations should be born in mind. Overall the PCO C/Es are larger than those from the RTO and ATO experiments but the C/E differences seem reasonable.

To summarize the PCO results, representative core region C/Es from each experiment are given in Table 10.13. The sodium (PCO-8-9) results indicate that accounting for local flux distortions and the adjoint heterogeneity error can greatly improve at least the scattering worth $\mathrm{C} / \mathrm{Es}$. Considering that the calculations are preliminary, the C/Es, although large, are not disturbing.

\subsection{Axial Expansion Oscillator Experiments}

Three measurements were made with the axial expansion oscillator, which are referred to as AEO-1, AEO-2 and AEO-3. All of the locations (see Fig. 10.1) had DFC core cells.

The expansion worths were computed by FOP with nodal diffusion theory. Cell average cross sections were used since the worth of unit cell motion was being computed. For each material that moved - core, axial blanket and reflector - the material worth in each axial node at the oscillator matrix position was calculated. A utility program fit the cumulative integral of a material's worth along this traverse to a cubic spline and then interpolated to get the integrals over the axial intervals where that material changed. (This is the same interpolation scheme that was used for the RTO, ATO and PCO calculations.)

The results are shown in Table 10.14. The expansion worth is about half as large for AEO-1 even though the material motion was the same in all three experiments. This because the internal blanket at this location flattens the flux gradients. Another effect of the internal blanket is that motion of core material did not dominate the net calculated worth as much as in the other cases. The calculations appear to handle the internal blanket effects well, since all the C/Es are near unity and agree with each other to within the experimental uncertainty (3\%). 


\subsection{Bowing Oscillator Experiment}

The bowing oscillator (BO) shifted the core segment of a drawer radially a distance typical of power reactor bowing motion. The displacement was essentially uniform over the axial extent of the core segment; the effective average displacement, which was used here, was $0.133 \mathrm{~cm}$. The motion occurred in a steep radial flux gradient - one drawer from the radial blanket on the $Y$ axis (see Fig. 10.1). The experimental worth is $-0.01392 \phi$ and has a total uncertainty of $3.5 \%$.

C/Es were obtained by several methods. All of them used FOP, which should be valid for such a small perturbation. First a basic calculated worth was obtained by nodal diffusion theory with the XYZ geometry critical reference model. The resulting $C / E$ is 1.33 . Then $Y Z$ geometry models were used to calculate a number of improvements to the basic $\mathrm{C} / \mathrm{E}$. The $\mathrm{YZ}$ models represented matrix column 49 of the reactor explicitly and used group- and region-dependent $D^{2}$ values from the XYZ solutions to represent the $X$ dimension. Because the shifted material was quite similar to a normal DFC cell, standard DFC cell average microscopic cross sections were used in all the calculations.

The adjustments to the basic C/E are shown in Table 10.15. Since the bowing drawer composition matched the standard DFC cell composition reasonably well, there was little improvement from using the exact composition in the flux and adjoint computations. There is also little effect on the drawer-average flux and adjoint from halving the Y-direction node spacing. Replacing diffusion perturbation theory with $\mathrm{S}_{4}$ transport perturbation theory calculations made the $\mathrm{C} / \mathrm{E}$ larger by $3.2 \%$.

Since there was material motion within a $Y$ node interval and only node average (or node integrated) worths are known, the worth variation within a node must be inferred to get the bowing motion worth. Several schemes to do this were tried, and in each of them it was assumed that the known average worth equals the worth at the $\mathrm{Y}$-center of the node. The scheme used for the basic $\mathrm{C} / \mathrm{E}$, called full-cell 1 inear interpolation, was to linearly interpolate between mesh centers. The full cell designation refers 
to the $\mathrm{Y}$-interval from which the average is taken being one cell (drawer) wide. Half-cell linear interpolation is the same as the basic method except that there are 2 nodes per drawer in $Y$. Half-cell linear extrapolation is the new ANL design method due to Finck ${ }^{21}$. It differs by extrapolating the linear variation between mesh centers to the edges of the cell in which the bowing occurs. The last alternative shown in Table 10.15 assumes the worth is constant within the half-cell wide node interval. It is the old design method, and gives an unreasonably low C/E.

The final C/E for the bowing oscillator experiment uses the first three adjustments of Table 10.15 and the current design interpolation scheme (half-cell linear extrapolation). The resulting $\mathrm{C} / \mathrm{E}$ is 1.26 .

\subsection{Al1-Plate Shifting Experiment}

In this experiment plate columns of a cell were rearranged such that most of the cell moved radially a small distance. To achieve this it was necessary to move a narrow, low worth section of the cell a much larger distance in the opposite direction. To make the shift look as much as possible like bowing, the only material moving counter to the rest of the cell was a $0.64 \mathrm{~cm}$-wide clad sodium column. The shifting occurred in a zone of cells that spanned much of the radial extent of the outer core (see

Fig. 10.1). The zone had the normal mix of SFC and DFC cells, but the cells were modified to have $0.64 \mathrm{~cm}$-wide sodium columns at the cell edges. The experimental worth is -6.02 cents and is uncertain by $3.1 \%$. As in the bowing oscillator analysis a basic solution was obtained and then some refinements were made. For these calculations the exact reactor configuration used in the experiment was modeled to get the flux and adjoint. Anticipating the use of the various interpolation schemes described in Section 10.6, the XYZ geometry model had a half-drawer node spacing in the $X$ direction over the range of the shifting zone. The worths were computed by FOP in nodal diffusion theory. In the basic calculation the standard SFC and DFC microscopic cross sections were used to compute the material worths. The basic calculation used half-cell linear interpolation and yielded a $\mathrm{C} / \mathrm{E}$ of 1.17 . 
The main calculational refinement was to account for the change in the within-cell flux distribution that occurred when the plate columns were rearranged. This was done by computing cell average cross sections for each of the pre-shift and post-shift cell types. Group-dependent bucklings for that drawer type in the zone, obtained from the XYZ geometry nodal diffusion flux calculation, were used in this cross section processing. The reactivity effect due to changing from pre-shift to post-shift microscopic cross sections was calculated to be +1.01 cents. This reduces the $\mathrm{C} / \mathrm{E}$ from 1.17 to 1.00 .

The last adjustment was to use the new design method interpolation scheme, half-cell linear extrapolation. This changed the calculated worth by only $1 \%$, yielding a final $\mathrm{C} / \mathrm{E}$ of 1.01 .

This C/E is considerably lower than the final C/E from the bowing oscillator experiment, even though the bowing oscillator was at a radius encompassed by the plate shifting zone. This C/E variation is believed to be due partly to the difficulty of calculating gradients induced by the mix of SFC and DFC cells, which occurs in ZPPR assemblies but not power reactors. Another factor contributing to the $C / E$ difference is the difference in the combination of diluent and fuel motion that occurred in the two kinds of experiments. Also, $3 \%$ of the difference is attributable to using a transport correction for only the bowing oscillator calculation. 
TABLE 10.1 Description of ZPPR-17A Oscillator Measurements

\begin{tabular}{|c|c|c|c|}
\hline Name & Oscillator & Sample & $\begin{array}{c}\text { Matrix } \\
\text { Position } \\
\end{array}$ \\
\hline ATO-1 & Axial Tube & $\begin{array}{l}\text { PU-30 } \\
\text { DU-6 } \\
B-1 \\
D-14 \\
S S-1 \\
U-6\end{array}$ & $159-49 \quad(D)$ \\
\hline ATO-2 & Axial Tube & $\begin{array}{l}P U-30 \\
D U-6 \\
B-1 \\
D-14 \\
S S-1 \\
U-6\end{array}$ & $163-41$ (E) \\
\hline ATO -3 & Axial Tube & $\begin{array}{l}S S-1 \\
D U-6 \\
D-14 \\
P U-30 \\
B-1\end{array}$ & $171-49(G)$ \\
\hline RTO & Radial Tube & $\begin{array}{l}\text { PU-30 } \\
\text { DU-6 } \\
B-1 \\
D-14 \\
\text { SS-1 } \\
C f \text { @ } 3 \mathrm{~W} \\
\text { Cf e } 80 \mathrm{~W}\end{array}$ & $1,248()$. \\
\hline AEO-1 & Axial Expan. & I.C. DFC & $138-49$ (D) \\
\hline $\mathrm{AEO}-2$ & Axial Expan. & O.C. DFC & $163-41$ (E) \\
\hline $\mathrm{AEO}-3$ & Axial Expan. & O.C. DFC & $166-55$ (E) \\
\hline BO & Bowing & $\begin{array}{l}\text { O.C. DFC } \\
\text { repeat }\end{array}$ & $126-49(G)$ \\
\hline $\mathrm{PCO}-1$ & Plate Column & $\mathrm{Pu}-\mathrm{U}+\mathrm{SS}$ & $147-33$ (B) \\
\hline $\mathrm{PCO}-2$ & Plate Column & $\mathrm{UO}_{0}$ & $147-33$ (B) \\
\hline $\mathrm{PCO}-3$ & Plate Column & $\mathrm{Pu}-\mathrm{U}^{3} \stackrel{8}{+} \mathrm{SS}$ & $149-40$ (A) \\
\hline $\mathrm{PCO}-4$ & Plate Column & $\mathrm{U}_{3} \mathrm{O}_{4}$ & $149-40$ (A) \\
\hline $\mathrm{PCO}-5$ & Plate Column & $P u^{3}-0$ & $163-41$ (E) \\
\hline PCO-6 & Plate Column & $\mathrm{Pu}-\mathrm{U}$ & $148-31$ (C) \\
\hline $\mathrm{PCO}-7$ & Plate Column & $\mathrm{U}_{3} \mathrm{O}_{4}$ & $148-31$ (C) \\
\hline PCO-8-9 & Plate Column & $\mathrm{Na}$ & $148-68$ (C) \\
\hline PCO-9 & Plate Column & Void Cans & $148-68$ (C) \\
\hline PCO -10 & Plate Column & $\mathrm{Fe}_{2} \mathrm{O}_{3}$ & $148-31$ (C) \\
\hline
\end{tabular}

Location symbol for Figure 10.1 is in parentheses. 
TABLE 10.2 PU-30, RTO Worth Traverse

\begin{tabular}{|c|c|c|c|c|c|c|c|c|c|c|c|c|c|}
\hline \multicolumn{3}{|c|}{ Position } & \multirow[b]{2}{*}{ Experimental } & \multirow[b]{2}{*}{ Worth ${ }^{\mathrm{a}}($ cents $)$} & \multirow{2}{*}{$\begin{array}{c}\text { Initial } \\
\mathrm{C} / \mathrm{E}^{\mathrm{b}} \\
\end{array}$} & \multicolumn{4}{|c|}{ Calculation Correction Factors } & \multicolumn{4}{|c|}{ Final Results } \\
\hline \# & $\underline{\text { Regn }}$ & $\underline{x(\mathrm{~cm})}$ & & & & Size & Transp & Position & Adj Het & $\mathrm{C}($ cents $)$ & $C-E$ & & $C / E^{b}$ \\
\hline 49 & IBKT & 2.77 & $0.2195 \pm 0.0020$ & $0.98)$ & 0.99 & 1.000 & 0.942 & 1.005 & 0.997 & 0.2060 & -0.0135 & 0.938 & \pm 0.008 \\
\hline 48 & IBKT & 8.29 & $0.2210 \pm 0.0018$ & $0.8 \%)$ & 0.99 & 1.000 & 0.943 & 1.005 & 0.997 & 0.2074 & -0.0136 & 0.938 & \pm \pm 0.008 \\
\hline 47 & IBKT & 13.82 & $0.2259 \pm 0.0018$ & $0.8 \%)$ & 0.98 & 1.000 & 0.943 & 1.005 & 0.997 & 0.2098 & -0.0161 & 0.929 & \pm 0.007 \\
\hline 46 & IBKT & 19.34 & $0.2263 \pm 0.0022$ & $1.0 \%)$ & 1.00 & 1.000 & 0.943 & 1.005 & 0.997 & 0.2136 & -0.0127 & 0.944 & \pm 0.009 \\
\hline 45 & IBKT & 24.86 & $0.2330 \pm 0.0021$ & $0.9 \%)$ & 0.99 & 1.000 & 0.942 & 1.005 & 0.997 & 0.2189 & -0.0141 & 0.940 & \pm 0.008 \\
\hline 44 & IBKT & 30.39 & $0.2386 \pm 0.0021$ & $0.9 \%)$ & 1.00 & 1.000 & 0.941 & 1.005 & 0.997 & 0.2258 & -0.0128 & 0.946 & \pm 0.009 \\
\hline 43 & IBKT & 35.91 & $0.2512 \pm 0.0022$ & $0.98)$ & 0.99 & 1.000 & 0.940 & 1.006 & 0.997 & 0.2345 & -0.0167 & 0.934 & \pm 0.008 \\
\hline 42 & IBKT & 41.44 & $0.2541 \pm 0.0021$ & $0.8 \%)$ & 1.02 & 1.000 & 0.939 & 1.006 & 0.997 & 0.2454 & -0.0086 & 0.966 & \pm 0.008 \\
\hline 41 & IBKT & 46.96 & $0.2716 \pm 0.0019$ & $0.7 \%)$ & 1.01 & 1.000 & 0.938 & 1.007 & 0.997 & 0.2586 & -0.0129 & 0.952 & \pm 0.007 \\
\hline 40 & IBKT & 52.49 & $0.2871 \pm 0.0021$ & $0.7 \%)$ & 1.02 & 1.000 & 0.937 & 1.007 & 0.997 & 0.2748 & -0.0123 & 0.957 & \pm 0.007 \\
\hline 39 & IBKT & 58.01 & $0.3007 \pm 0.0017$ & $0.6 \%)$ & 1.04 & 1.000 & 0.937 & 1.008 & 0.997 & 0.2942 & -0.0065 & 0.978 & \pm 0.006 \\
\hline 38 & IBKT & 63.53 & $0.3186 \pm 0.0020$ & $( \pm 0.6 \%)$ & 1.06 & 1.000 & 0.936 & 1.009 & 0.997 & 0.3173 & -0.0013 & 0.996 & \pm 0.006 \\
\hline 37 & IBKT & 69.05 & $0.3319 \pm 0.0021$ & $( \pm 0.6 \%)$ & 1.10 & 1.000 & 0.935 & 1.008 & 0.997 & 0.3442 & 0.0122 & 1.037 & \pm 0.007 \\
\hline 36 & IBKT & 74.59 & $0.3527 \pm 0.0019$ & $( \pm 0.5 \%)$ & 1.13 & 1.000 & 0.934 & 1.008 & 0.997 & 0.3754 & 0.0227 & 1.064 & \pm 0.006 \\
\hline 35 & IBKT & 80.11 & $0.3728 \pm 0.0021$ & $( \pm 0.6 \%)$ & 1.18 & 1.000 & 0.933 & 1.006 & 0.997 & 0.4119 & 0.0391 & 1.105 & \pm 0.006 \\
\hline 34 & IBKT & 85.63 & $0.3914 \pm 0.0022$ & $( \pm 0.6 \%)$ & 1.25 & 1.000 & 0.946 & 1.003 & 0.997 & 0.4641 & 0.0727 & 1.186 & \pm 0.007 \\
\hline 33 & DFC & 91.15 & $0.4067 \pm 0.0017$ & $( \pm 0.4 \%)$ & 1.10 & 1.046 & 0.976 & 1.002 & 0.972 & 0.4444 & 0.0377 & 1.093 & \pm 0.005 \\
\hline 32 & SFC & 96.68 & $0.4090 \pm 0.0024$ & $( \pm \quad 0.6 \%)$ & 1.11 & 1.051 & 0.986 & 1.003 & 0.959 & 0.4522 & 0.0432 & 1.106 & \pm 0.006 \\
\hline 31 & SFC & 102.21 & $0.3974 \pm 0.0021$ & $( \pm 0.5 \%)$ & 1.11 & 1.055 & 0.987 & 1.005 & 0.959 & 0.4410 & 0.0436 & 1.110 & \pm 0.006 \\
\hline 30 & DFC & 107.73 & $0.3666 \pm 0.0020$ & $0.5 \%)$ & 1.09 & 1.059 & 0.988 & 1.006 & 0.972 & 0.4101 & 0.0436 & 1.119 & \pm 0.006 \\
\hline 29 & SFC & 113.26 & $0.3089 \pm 0.0024$ & $0.8 \%)$ & 1.11 & 1.056 & 0.989 & 1.007 & 0.959 & 0.3461 & 0.0372 & 1.120 & \pm 0.009 \\
\hline 28 & $\mathrm{SFC}$ & 118.78 & $0.2526 \pm 0.0026$ & $1.0 \%)$ & 1.11 & 1.055 & 0.989 & 1.008 & 0.959 & 0.2814 & 0.0288 & 1.114 & \pm 0.012 \\
\hline 27 & DFC & 124.30 & $0.1903 \pm 0.0022$ & $1.2 \%)$ & 1.09 & 1.055 & 0.986 & 1.009 & 0.972 & 0.2123 & 0.0221 & 1.116 & \pm 0.013 \\
\hline 26 & SFC & 129.83 & $0.1370 \pm 0.0020$ & $1.5 \%)$ & 1.07 & 1.041 & 0.969 & 1.009 & 0.959 & 0.1430 & 0.0060 & 1.044 & \pm 0.015 \\
\hline 25 & RBKT & 135.35 & $0.0875 \pm 0.0020$ & $( \pm 2.3 \%)$ & 1.24 & 1.000 & 0.940 & 1.009 & 1.000 & 0.1030 & 0.0155 & 1.177 & \pm 0.027 \\
\hline 24 & RBKT & 140.88 & $0.0559 \pm 0.0022$ & $( \pm 4.0 \%)$ & 1.03 & 1.000 & 0.929 & 1.010 & 1.000 & 0.0538 & -0.0021 & 0.962 & \pm 0.038 \\
\hline 23 & RBKT & 146.40 & $0.0312 \pm 0.0021$ & $( \pm 6.8 \%)$ & 0.94 & 1.000 & 0.930 & 1.010 & 1.000 & 0.0274 & -0.0038 & 0.878 & \pm 0.060 \\
\hline 22 & RBKT & 151.92 & $0.0178 \pm 0.0023$ & $( \pm 12.8 \%)$ & 0.82 & 1.000 & 0.935 & 1.010 & 1.000 & 0.0137 & -0.0041 & 0.771 & \pm 0.099 \\
\hline 21 & RBKT & 157.45 & $0.0072 \pm 0.0020$ & $( \pm 28.1 \%)$ & - & 1.000 & 0.942 & 1.010 & 1.000 & 0.0070 & -0.0001 & & $=$ \\
\hline 20 & RREF & 162.97 & $0.0071 \pm 0.0021$ & $( \pm 29.8 \%)$ & - & 1.000 & 0.937 & 1.010 & 1.000 & 0.0036 & -0.0036 & & - \\
\hline
\end{tabular}

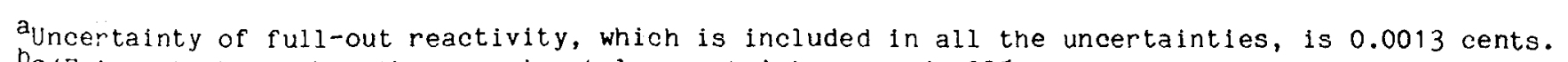

${ }^{\mathrm{O}} \mathrm{C} / \mathrm{E}$ is not shown when the experimental uncertainty exceeds $20 \%$. 
TABLE 10.3 DU-6, RTO worth Traverse

\begin{tabular}{|c|c|c|c|c|c|c|c|c|c|c|c|c|c|}
\hline \multicolumn{3}{|c|}{ Position } & \multirow[b]{2}{*}{ Experimental } & \multirow{2}{*}{ Worth ${ }^{a}$ (cents) } & \multirow{2}{*}{$\begin{array}{l}\text { Initial } \\
\mathrm{C} / \mathrm{E}^{\mathrm{b}} \\
\end{array}$} & \multicolumn{4}{|c|}{ Calculation Correction Factors } & \multicolumn{4}{|c|}{ Final Results } \\
\hline$\#$ & Regn & $X(\mathrm{~cm})$ & & & & Size & Transp & Position & Adj Het & $\mathrm{C}$ (cents) & $\mathrm{C}-\mathrm{E}$ & & $\mathrm{C} / \mathrm{E}^{\mathrm{b}}$ \\
\hline 49 & IBKT & 2.76 & $-0.0121 \pm 0.0009$ & $7.6 \%)$ & 0.84 & 0.992 & 0.981 & 0.966 & 0.999 & -0.0096 & 0.0025 & 0.793 & \pm 0.060 \\
\hline 48 & IBKT & 8.30 & $-0.0107 \pm 0.0010$ & $9.5 \%)$ & 0.96 & 0.992 & 0.982 & 0.966 & 0.999 & -0.0096 & 0.0010 & 0.902 & \pm 0.086 \\
\hline 47 & IBKT & 13.82 & $-0.0128 \pm 0.0012$ & $( \pm 9.2 \%)$ & 0.81 & 0.992 & 0.981 & 0.966 & 0.999 & -0.0098 & 0.0030 & 0.763 & \pm 0.070 \\
\hline 46 & IBKT & 19.34 & $-0.0122 \pm 0.0009$ & $( \pm 7.4 \%)$ & 0.87 & 0.992 & 0.981 & 0.966 & 0.999 & -0.0099 & 0.0023 & 0.812 & \pm 0.060 \\
\hline 45 & IBKT & 24.86 & $-0.0107 \pm 0.0011$ & $( \pm 9.9 \%)$ & 1.01 & 0.992 & 0.980 & 0.966 & 0.999 & -0.0101 & 0.0006 & 0.947 & \pm 0.094 \\
\hline 44 & IBKT & 30.39 & $-0.0114 \pm 0.0010$ & $8.9 \%)$ & 0.98 & 0.992 & 0.978 & 0.966 & 0.999 & -0.0105 & 0.0009 & 0.920 & \pm 0.082 \\
\hline 43 & IBKT & 35.91 & $-0.0128 \pm 0.0010$ & $8.1 \%)$ & 0.91 & 0.992 & 0.977 & 0.966 & 0.999 & -0.0108 & 0.0019 & 0.850 & \pm 0.068 \\
\hline 42 & IBKT & 41.44 & $-0.0140 \pm 0.0009$ & $( \pm 6.5 \%)$ & 0.86 & 0.992 & 0.975 & 0.966 & 0.999 & -0.0113 & 0.0027 & 0.807 & \pm 0.052 \\
\hline 41 & IBKT & 46.96 & $-0.0142 \pm 0.0011$ & $( \pm 7.5 \%)$ & 0.90 & 0.993 & 0.973 & 0.967 & 0.999 & -0.0119 & 0.0023 & 0.841 & \pm 0.063 \\
\hline 40 & IBKT & 52.48 & $-0.0120 \pm 0.0010$ & $( \pm 8.5 \%)$ & 1.13 & 0.993 & 0.971 & 0.967 & 0.999 & -0.0127 & -0.0007 & 1.055 & \pm 0.090 \\
\hline 39 & IBKT & 58.01 & $-0.0154 \pm 0.0010$ & $6.8 \%)$ & 0.95 & 0.993 & 0.968 & 0.967 & 0.999 & -0.0136 & 0.0018 & 0.884 & \pm 0.060 \\
\hline 38 & IBKT & 63.54 & $-0.0167 \pm 0.0010$ & $6.36)$ & 0.96 & 0.993 & 0.966 & 0.968 & 0.999 & -0.0148 & 0.0018 & 0.890 & \pm 0.056 \\
\hline 37 & IBKT & 69.06 & $-0.0174 \pm 0.0011$ & $6.4 \%)$ & 1.02 & 0.993 & 0.964 & 0.972 & 0.999 & -0.0164 & 0.0010 & 0.944 & \pm 0.060 \\
\hline 36 & IBK T & 74.59 & $-0.0202 \pm 0.0010$ & $4.9 \%)$ & 0.98 & 0.994 & 0.964 & 0.977 & 0.999 & -0.0184 & 0.0018 & 0.912 & \pm 0.045 \\
\hline 35 & IBKT & 80.11 & $-0.0203 \pm 0.0010$ & $5.0 \%)$ & 1.09 & 0.994 & 0.970 & 0.983 & 0.999 & -0.0210 & -0.0007 & 1.033 & \pm 0.052 \\
\hline 34 & IBKT & 85.63 & $-0.0229 \pm 0.0011$ & $4.9 \%)$ & 1.08 & 0.996 & 0.981 & 0.990 & 0.999 & -0.0240 & -0.0010 & 1.045 & \pm 0.051 \\
\hline 33 & DFC & 91.15 & $-0.0257 \pm 0.0010$ & $3.7 \%)$ & 1.06 & 0.999 & 0.996 & 0.998 & 0.947 & -0.0256 & 0.0001 & 0.997 & \pm 0.037 \\
\hline 32 & SFC & 96.68 & $-0.0270 \pm 0.0010$ & $3.8 \%)$ & 1.04 & 1.001 & 1.006 & 1.004 & 0.972 & -0.0276 & -0.0006 & 1.022 & \pm 0.038 \\
\hline 31 & $\mathrm{SFC}$ & 102.20 & $-0.0257 \pm 0.0011$ & $( \pm 4.4 \%)$ & 1.04 & 1.003 & 1.011 & 1.007 & 0.972 & -0.0265 & -0.0008 & 1.032 & \pm 0.046 \\
\hline 30 & DFC & 107.73 & $-0.0213 \pm 0.0010$ & $( \pm 4.6 \%)$ & 1.07 & 1.004 & 1.014 & 1.009 & 0.947 & -0.0223 & -0.0010 & 1.045 & \pm 0.048 \\
\hline 29 & SFC & 113.26 & $-0.0169 \pm 0.0011$ & $( \pm 6.5 \%)$ & 1.07 & 1.003 & 1.017 & 1.011 & 0.972 & -0.0181 & -0.0012 & 1.072 & \pm 0.069 \\
\hline 28 & $\mathrm{SFC}$ & 118.78 & $-0.0086 \pm 0.0011$ & $( \pm 12.3 \%)$ & 1.56 & 1.003 & 1.019 & 1.011 & 0.972 & -0.0135 & -0.0049 & 1.565 & \pm 0.192 \\
\hline 27 & DFC & 124.30 & $-0.0058 \pm 0.0010$ & $( \pm 17.2 \%)$ & 1.34 & 1.002 & 1.021 & 1.012 & 0.947 & -0.0076 & -0.0018 & 1.310 & \pm 0.225 \\
\hline 26 & SFC & 129.83 & $0.0015 \pm 0.0010$ & $( \pm 63.0 \%)$ & - & 0.999 & 1.041 & 1.014 & 0.972 & -0.0030 & -0.0045 & & - \\
\hline 25 & $\mathrm{RBKT}$ & 135.35 & $0.0026 \pm 0.0010$ & $( \pm 39.7 \%)$ & - & 0.996 & 1.229 & 1.016 & 1.000 & -0.0009 & -0.0035 & & - \\
\hline 24 & RBKT & 140.88 & $0.0027 \pm 0.0010$ & $( \pm 37.9 \%)$ & - & 0.994 & 1.332 & 1.017 & 1.000 & -0.0004 & -0.0031 & & - \\
\hline 23 & RBKT & 146.40 & $0.0006 \pm 0.0009$ & $( \pm 168.2 \%)$ & - & 0.993 & 1.356 & 1.017 & 1.000 & -0.0001 & -0.0007 & & - \\
\hline 22 & RBKT & 151.92 & $-0.0016 \pm 0.0011$ & $( \pm 67.8 \%)$ & - & 0.992 & 1.253 & 1.018 & 1.000 & -0.0001 & 0.0015 & & - \\
\hline 21 & RBKT & 157.45 & $-0.0019 \pm 0.0011$ & $( \pm 57.2 \%)$ & - & 0.991 & 1.074 & 1.014 & 1.000 & 0.0000 & 0.0019 & & - \\
\hline 20 & RREF & 162.98 & $0.0006 \pm 0.0011$ & $( \pm 167.3 \%)$ & - & 0.991 & 0.992 & 1.012 & 1.000 & 0.0000 & -0.0007 & & - \\
\hline
\end{tabular}

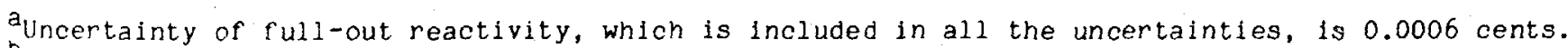

${ }^{\mathrm{b}} \mathrm{C} / \mathrm{E}$ is not shown when the experimental uncertainty exceeds $20 \%$. 
TABLE 10.4 PU-30, ATO-1 Worth Traverse

\begin{tabular}{|c|c|c|c|c|c|c|c|c|c|c|c|c|c|}
\hline \multirow[b]{2}{*}{$\#$} & \multicolumn{2}{|c|}{ Position } & \multirow{2}{*}{\multicolumn{2}{|c|}{ Experimental Worth ${ }^{a}$ (cents) }} & \multirow{2}{*}{$\begin{array}{c}\text { Initial } \\
\mathrm{C} / \mathrm{E} \\
\end{array}$} & \multicolumn{4}{|c|}{ Calculation Correction Factors } & \multicolumn{4}{|c|}{ Final Results } \\
\hline & Regn & $\mathrm{X}(\mathrm{cm})$ & & & & Size & Transp & Position & Adj Het & $\mathrm{C}$ (cents) & $\mathrm{C}-\mathrm{E}$ & & $C / E$ \\
\hline 1 & IBKT & 3.99 & $0.2864 \pm 0.0023$ & $0.8 \%)$ & 1.07 & 1.000 & 0.937 & 0.997 & 0.997 & 0.2866 & 0.0002 & 1.001 & \pm 0.008 \\
\hline 2 & IBKT & 7.62 & $0.2872 \pm 0.0024$ & $0.8 \%)$ & 1.12 & 0.996 & 0.939 & 0.997 & 1.000 & 0.2993 & 0.0122 & 1.042 & \pm 0.009 \\
\hline 3 & IBKT & 12.70 & $0.2966 \pm 0.0025$ & $0.9 \%)$ & 1.13 & 1.003 & 0.952 & 0.997 & 0.996 & 0.3171 & 0.0205 & 1.069 & \pm 0.009 \\
\hline 4 & DFC & 17.78 & $0.3146 \pm 0.0025$ & $0.8 \%)$ & 1.04 & 1.029 & 0.979 & 0.999 & 0.980 & 0.3241 & 0.0095 & 1.030 & \pm 0.008 \\
\hline 5 & DFC & 22.87 & $0.3246 \pm 0.0024$ & $0.8 \%)$ & 0.98 & 1.051 & 1.000 & 1.001 & 0.969 & 0.3242 & -0.0004 & 0.999 & \pm 0.008 \\
\hline 6 & DFC & 27.95 & $0.3277 \pm 0.0025$ & $0.8 \%)$ & 0.94 & 1.059 & 1.008 & 1.001 & 0.967 & 0.3187 & -0.0090 & 0.972 & \pm 0.007 \\
\hline 7 & DFC & 33.02 & $0.3088 \pm 0.0026$ & $0.9 \%)$ & 0.96 & 1.058 & 1.006 & 1.001 & 0.971 & 0.3053 & -0.0035 & 0.989 & \pm 0.008 \\
\hline 8 & $\mathrm{DFC}$ & 38.10 & $0.2726 \pm 0.0024$ & $0.9 \%)$ & 0.99 & 1.055 & 1.002 & 1.000 & 0.973 & 0.2763 & 0.0037 & 1.013 & \pm 0.009 \\
\hline 9 & DFC & 43.18 & $0.2295 \pm 0.0024$ & $1.1 \%)$ & 0.98 & 1.052 & 0.996 & 1.000 & 0.971 & 0.2297 & 0.0002 & 1.001 & \pm 0.011 \\
\hline 10 & DFC & 48.26 & $0.1782 \pm 0.0029$ & $1.6 \%)$ & 0.98 & 1.043 & 0.984 & 1.000 & 0.972 & 0.1745 & -0.0037 & 0.979 & \pm 0.016 \\
\hline 11 & $\mathrm{ABKT}$ & 53.34 & $0.1286 \pm 0.0022$ & $1.7 \%)$ & 1.07 & 1.014 & 0.958 & 1.000 & 0.990 & 0.1331 & 0.0045 & 1.035 & \pm 0.018 \\
\hline 12 & ABKT & 58.42 & $0.0977 \pm 0.0027$ & $( \pm 2.8 \%)$ & 1.08 & 0.994 & 0.940 & 1.000 & 1.004 & 0.0989 & 0.0012 & 1.012 & \pm 0.028 \\
\hline 13 & $\mathrm{ABKT}$ & 63.50 & $0.0664 \pm 0.0026$ & $4.0 \%)$ & 1.12 & 0.991 & 0.937 & 1.000 & 1.006 & 0.0688 & 0.0024 & 1.036 & \pm 0.041 \\
\hline 14 & ABKT & 68.58 & $0.0423 \pm 0.0025$ & $( \pm 5.9 \%)$ & 1.11 & 0.998 & 0.944 & 1.000 & 1.001 & 0.0441 & 0.0018 & 1.043 & \pm 0.061 \\
\hline 15 & ABKT & 73.66 & $0.0346 \pm 0.0023$ & $( \pm 6.8 \%)$ & 0.82 & 1.002 & 0.949 & 1.000 & 0.998 & 0.0272 & -0.0074 & 0.785 & \pm 0.053 \\
\hline 16 & ABKT & 78.75 & $0.0216 \pm 0.0027$ & $( \pm 12.7 \%)$ & 0.86 & 1.002 & 0.951 & 1.000 & 0.999 & 0.0181 & -0.0035 & 0.837 & \pm 0.106 \\
\hline 17 & AREF & 83.82 & $0.0149 \pm 0.0023$ & $( \pm 15.4 \%)$ & 0.94 & 1.000 & 0.953 & 1.000 & 1.000 & 0.0134 & -0.0015 & 0.900 & \pm 0.139 \\
\hline
\end{tabular}

ancertainty of full-out reactivity, which is included in all the uncertainties, is 0.0015 cents. 
TABLE 10.5 B-1, ATO-1 Worth Traverse

\begin{tabular}{|c|c|c|c|c|c|c|c|c|c|c|c|c|}
\hline \multicolumn{3}{|c|}{ Position } & & \multirow{2}{*}{$\begin{array}{l}\text { Intial } \\
\mathrm{C} / \mathrm{E}^{\mathrm{b}} \\
\end{array}$} & \multicolumn{4}{|c|}{ Calculation Correction Factors } & \multicolumn{4}{|c|}{ Final Results } \\
\hline \# & $\underline{R e g n}$ & $\underline{X(\mathrm{~cm})}$ & Worth ${ }^{\mathrm{a}}$ (cents) & & Size & Transp & Position & Adj Het & $\mathrm{C}$ (cents) & $C-E$ & & $/ E^{b}$ \\
\hline 1 & IBKT & 3.99 & $-0.2486 \pm 0.0024( \pm 1.0 \%)$ & 1.42 & 0.703 & 0.969 & 1.003 & 1.000 & -0.2407 & 0.0079 & $0.968 \pm$ & \pm 0.009 \\
\hline 2 & IBKT & 7.62 & $-0.2718 \pm 0.0026( \pm 1.04)$ & 1.43 & 0.696 & 0.972 & 1.004 & 1.000 & -0.2631 & 0.0087 & $0.968 \pm$ & \pm 0.009 \\
\hline 3 & IBKT & 12.70 & $-0.3213 \pm 0.0028( \pm 0.9 \%)$ & 1.39 & 0.705 & 0.980 & 1.003 & 1.000 & -0.3095 & 0.0118 & $0.963 \pm$ & \pm 0.008 \\
\hline 4 & DFC & 17.78 & $-0.3810 \pm 0.0026( \pm 0.7 \%)$ & 1.28 & 0.745 & 0.990 & 0.997 & 0.998 & -0.3596 & 0.0215 & $0.944 \pm$ & \pm 0.007 \\
\hline 5 & DFC & 22.86 & $-0.3971 \pm 0.0027$ & 1.27 & 0.780 & 0.998 & 0.992 & 0.997 & -0.3880 & 0.0091 & $0.977 \pm$ & \pm 0.007 \\
\hline 6 & DFC & 27.94 & $-0.3954 \pm 0.0027( \pm 0.7 \%)$ & 1.24 & 0.798 & 1.003 & 0.991 & 0.997 & -0.3878 & 0.0076 & $0.981 \pm$ & \pm 0.007 \\
\hline$?$ & DFC & 33.02 & $-0.3720 \pm 0.0028( \pm 0.8 \%)$ & 1.22 & 0.802 & 1.005 & 0.993 & 0.997 & -0.3618 & 0.0103 & $0.972 \pm$ & \pm 0.007 \\
\hline 8 & DFC & 38.10 & $-0.3301 \pm 0.0027( \pm 0.8 \%)$ & 1.21 & 0.800 & 1.004 & 0.994 & 0.997 & -0.3191 & 0.0110 & $0.967 \pm$ & \pm 0.008 \\
\hline 9 & DFC & 43.18 & $-0.2653 \pm 0.0028( \pm 1.1 \%)$ & 1.25 & 0.793 & 1.001 & 0.994 & 0.997 & -0.2618 & 0.0035 & $0.987 \pm$ & \pm 0.010 \\
\hline 10 & DFC & 48.26 & $-0.2023 \pm 0.0029( \pm 1.4 \%)$ & 1.25 & 0.775 & 0.995 & 0.994 & 0.997 & -0.1935 & 0.0088 & $0.957 \pm$ & \pm 0.014 \\
\hline 11 & ABKT & 53.34 & $-0.1281 \pm 0.0024( \pm 1.9 \%)$ & 1.36 & 0.739 & 0.987 & 0.998 & 0.999 & -0.1268 & 0.0013 & $0.990 \pm$ & \pm 0.019 \\
\hline 12 & $A B K T$ & 58.42 & $-0.0762 \pm 0.0029( \pm 3.8 \%)$ & 1.44 & 0.712 & 0.980 & 1.001 & 1.000 & -0.0757 & 0.0005 & $0.993 \pm$ & \pm 0.037 \\
\hline 13 & ABKT & 63.50 & $-0.0407 \pm 0.0027( \pm 6.5 \%)$ & 1.62 & 0.703 & 0.973 & 1.001 & 1.001 & -0.0434 & -0.0027 & $1.065 \pm$ & \pm 0.070 \\
\hline 14 & ABKT & 68.58 & $-0.0272 \pm 0.0027( \pm 10.1 \%)$ & 1.44 & 0.698 & 0.969 & 1.000 & 1.000 & -0.0258 & 0.0014 & $0.948 \pm$ & \pm 0.095 \\
\hline 15 & ABKT & 73.67 & $-0.0123 \pm 0.0027( \pm 21.8 \%)$ & - & 0.672 & 0.966 & 1.000 & 1.000 & -0.0145 & -0.0022 & & - \\
\hline 15 & $\mathrm{ABKT}$ & 78.74 & $-0.0079 \pm 0.0030( \pm 37.7 \%)$ & - & 0.623 & 0.967 & 1.000 & 1.000 & -0.0084 & -0.0005 & & - \\
\hline 17 & AREF & 83.82 & $-0.0031 \pm 0.0025( \pm 81.8 \%)$ & - & 0.578 & 0.968 & 1.000 & 1.000 & -0.0053 & -0.0022 & & - \\
\hline
\end{tabular}

ancertainty of full-out reactivity, which is included in all the uncertainties, is 0.0017 cents.

$\mathrm{b} / \mathrm{f}$ is not shown when the experimental uncertainty exceeds $20 \%$. 
TABLE 10.6 U-6, ATO-1 Worth Traverse

\begin{tabular}{|c|c|c|c|c|c|c|c|c|c|c|c|c|c|}
\hline \multicolumn{3}{|c|}{ Position } & \multirow{2}{*}{\multicolumn{2}{|c|}{ Experimental worth ${ }^{2}$ (cents) }} & \multirow{2}{*}{$\begin{array}{c}\text { Initial } \\
\mathrm{C} / \mathrm{E} \\
\end{array}$} & \multicolumn{4}{|c|}{ Calculation Correction Factors } & \multicolumn{4}{|c|}{ Final Results } \\
\hline$\#$ & Regn & $x(\mathrm{~cm})$ & & & & Size & Transp & Position & Adj Het & c (cents) & $C-E$ & & $\mathrm{C} / \mathrm{E}$ \\
\hline 1 & IBKT & 3.99 & $0.3138 \pm 0.0024$ & $( \pm 0.8 \%)$ & 1.30 & 0.849 & 0.947 & 0.999 & 0.997 & 0.3255 & 0.0117 & 1.037 & \pm 0.008 \\
\hline 2 & IBKT & 7.62 & $0.3108 \pm 0.0023$ & $( \pm 0.7 \%)$ & 1.27 & 0.860 & 0.949 & 0.999 & 1.000 & 0.3226 & 0.0119 & 1.038 & \pm 0.008 \\
\hline 3 & IBKT & 12.71 & $0.3025 \pm 0.0024$ & $( \pm 0.8 \%)$ & 1.22 & 0.892 & 0.960 & 0.998 & 0.996 & 0.3153 & 0.0128 & 1.042 & \pm 0.008 \\
\hline 4 & DFC & 17.78 & $0.2920 \pm 0.0026$ & $( \pm 0.9 \%)$ & 1.16 & 0.940 & 0.984 & 0.996 & 0.978 & 0.3045 & 0.0125 & 1.043 & \pm 0.009 \\
\hline 5 & DFC & 22.86 & $0.2860 \pm 0.0026$ & $( \pm 0.9 \%)$ & 1.09 & 0.975 & 1.003 & 0.994 & 0.966 & 0.2928 & 0.0067 & 1.024 & \pm 0.009 \\
\hline 6 & DFC & 27.94 & $0.2813 \pm 0.0027$ & $( \pm 0.9 \%)$ & 1.04 & 0.991 & 1.011 & 0.994 & 0.963 & 0.2808 & -0.0005 & 0.998 & \pm 0.009 \\
\hline 7 & DEC & 33.02 & $0.2670 \pm 0.0027$ & $( \pm 1.0 \%)$ & 1.04 & 0.990 & 1.011 & 0.994 & 0.968 & 0.2662 & -0.0008 & 0.997 & \pm 0.010 \\
\hline 8 & $\mathrm{DFC}$ & 38.10 & $0.2335 \pm 0.0026$ & $( \pm 1.1 \%)$ & 1.08 & 0.985 & 1.007 & 0.995 & 0.970 & 0.2411 & 0.0077 & 1.033 & \pm 0.011 \\
\hline 9 & $\mathrm{DFC}$ & 43.18 & $0.2031 \pm 0.0027$ & $( \pm 1.3 \%)$ & 1.06 & 0.979 & 1.002 & 0.994 & 0.968 & 0.2038 & 0.0006 & 1.003 & \pm 0.013 \\
\hline 10 & DEC & 48.26 & $0.1617 \pm 0.0028$ & $( \pm 1.7 \%)$ & 1.08 & 0.962 & 0.991 & 0.994 & 0.969 & 0.1603 & -0.0015 & 0.991 & \pm 0.017 \\
\hline 11 & ABKT & 53.34 & $0.1298 \pm 0.0023$ & $( \pm 1.8 \%)$ & 1.11 & 0.917 & 0.967 & 0.998 & 0.989 & 0.1261 & -0.0036 & 0.972 & \pm 0.017 \\
\hline 12 & ABKT & 58.42 & $0.1010 \pm 0.0026$ & $( \pm 2.6 \%)$ & 1.16 & 0.873 & 0.951 & 1.001 & 1.004 & 0.0974 & -0.0035 & 0.965 & \pm 0.025 \\
\hline 13 & $\mathrm{ABKT}$ & 63.50 & $0.0739 \pm 0.0026$ & $( \pm 3.5 \%)$ & 1.23 & 0.840 & 0.948 & 1.001 & 1.007 & 0.0722 & -0.0017 & 0.977 & \pm 0.034 \\
\hline 14 & ABKT & 68.58 & $0.0491 \pm 0.0027$ & $( \pm 5.4 \%)$ & 1.37 & 0.807 & 0.953 & 1.000 & 1.002 & 0.0511 & 0.0020 & 1.041 & \pm 0.056 \\
\hline 15 & $\mathrm{ABKT}$ & 73.66 & $0.0366 \pm 0.0023$ & $( \pm 6.2 \%)$ & 1.37 & 0.752 & 0.957 & 1.000 & 0.998 & 0.0358 & -0.0008 & 0.977 & \pm 0.061 \\
\hline 16 & ABKT & 78.75 & $0.0258 \pm 0.0027$ & $( \pm 10.6 \%)$ & 1.59 & 0.672 & 0.958 & 1.000 & 0.998 & 0.0262 & 0.0004 & 1.016 & \pm 0.108 \\
\hline 17 & AREF & 83.82 & $0.0149 \pm 0.0026$ & $( \pm 17.4 \%)$ & 2.35 & 0.594 & 0.959 & 1.000 & 1.000 & 0.0200 & 0.0051 & 1.342 & \pm 0.234 \\
\hline
\end{tabular}

ancertainty of full-out reactivity, which is included in all the uncertainties, is 0.0015 cents. 
TABLE 10.7 Difference Between RTO and

ATO C/Es at Similar Positions

\begin{tabular}{|c|c|c|c|}
\hline $\begin{array}{l}\text { RTO POsition \# } \\
\text { ATO Traverse }\end{array}$ & $\begin{array}{r}39 \\
\text { ATO-1 } \\
\end{array}$ & $\begin{array}{r}33 \\
\text { ATO-2 } \\
\end{array}$ & $\begin{array}{c}27 \\
\text { ATO }-3 \\
\end{array}$ \\
\hline Sample & & C/E Difference & \\
\hline $\begin{array}{l}P U-30 \\
D U-6 \\
B-1\end{array}$ & $\begin{aligned}-0.023 & \pm 0.010 \\
& \pm 0.076 \pm 0.012\end{aligned}$ & $\begin{aligned} 0.043 & \pm 0.007 \\
-0.009 & \pm 0.082 \\
0.006 & \pm 0.006\end{aligned}$ & $\begin{aligned} 0.068 & \pm 0.015 \\
& - \\
0.000 & \pm 0.012\end{aligned}$ \\
\hline
\end{tabular}

${ }^{a}$ At axial position $\|^{1}$. 
TABLE 10.8 Region-Average C/Es From ZPPR-17A RTO and ATO Traverses

\begin{tabular}{|c|c|c|c|c|}
\hline \multirow[b]{2}{*}{ Sample } & \multirow[b]{2}{*}{ Traverse } & \multicolumn{3}{|c|}{ Region-Average $\mathrm{C} / \mathrm{E}$} \\
\hline & & $\begin{array}{r}\text { Internal } \\
\text { Blanket }\end{array}$ & Core & $\begin{array}{c}\text { Outer } \\
\text { Blanket }\end{array}$ \\
\hline$P U-30$ & RTO & 0.988 & 1.103 & 1.006 \\
\hline$P U-30$ & ATO-1 & 1.037 & 0.998 & 0.982 \\
\hline$P U-30$ & ATO -2 & - & 1.049 & 1.188 \\
\hline$P U-30$ & ATO -3 & - & 1.043 & 1.109 \\
\hline $\mathrm{DU}-6$ & RTO & 0.900 & 1.033 & - \\
\hline $\mathrm{DU}-6$ & ATO-2 & - & 1.023 & - \\
\hline$B-1$ & RTO & 0.890 & 1.026 & 1.085 \\
\hline$B-1$ & ATO-1 & 0.967 & 0.969 & 1.016 \\
\hline $\mathrm{B}-1$ & ATO-2 & - & 1.004 & 0.902 \\
\hline$B-1$ & ATO -3 & - & 1.037 & 1.099 \\
\hline $\mathrm{U}-6$ & ATO -1 & 1.039 & 1.013 & 0.986 \\
\hline$U-6$ & ATO-2 & - & 1.065 & 1.117 \\
\hline
\end{tabular}

C/Es with uncertainties exceeding $10 \%$ did not contribute to the averages. 
TABLE 10.9 Neutron Importance Radial Traverse ${ }^{a}$

\begin{tabular}{|c|c|c|c|c|c|c|c|c|}
\hline \multirow[b]{2}{*}{ \# } & \multicolumn{2}{|c|}{ Position } & \multirow[b]{2}{*}{ Experimental } & \multirow{2}{*}{$\begin{array}{c}\text { Transp. } \\
\text { Corr. } \\
\end{array}$} & \multirow{2}{*}{$\begin{array}{c}\text { Position } \\
\text { Corr. }\end{array}$} & \multirow{2}{*}{$\begin{array}{c}\text { Final } \\
\mathrm{C} \\
\end{array}$} & \multirow{2}{*}{\multicolumn{2}{|c|}{$C / E$}} \\
\hline & Regn & $\mathrm{X}(\mathrm{cm})$ & & & & & & \\
\hline 49 & IBKT & 2.77 & $0.631 \pm 0.030( \pm 4.8 \%)$ & 0.978 & 0.985 & 0.528 & 0.838 & \pm 0.040 \\
\hline 48 & IBKT & 8.29 & $0.652 \pm 0.033( \pm 5.1 \%)$ & 0.978 & 0.985 & 0.530 & 0.814 & \pm 0.042 \\
\hline 47 & IBKT & 13.82 & $0.610 \pm 0.033( \pm 5.5 \%)$ & 0.978 & 0.985 & 0.533 & 0.874 & \pm 0.048 \\
\hline 46 & IBKT & 19.34 & $0.611 \pm 0.033( \pm 5.5 \%)$ & 0.978 & 0.985 & 0.538 & 0.880 & \pm 0.048 \\
\hline 45 & IBKT & 24.86 & $0.633 \pm 0.033( \pm 5.3 \%)$ & 0.978 & 0.985 & 0.545 & 0.861 & \pm 0.045 \\
\hline 44 & IBKT & 30.39 & $0.623 \pm 0.033( \pm 5.4 \%)$ & 0.977 & 0.985 & 0.553 & 0.888 & \pm 0.048 \\
\hline 43 & IBKT & 35.91 & $0.645 \pm 0.033( \pm 5.2 \%)$ & 0.977 & 0.985 & 0.564 & 0.874 & \pm 0.045 \\
\hline 42 & IBKT & 41.44 & $0.653 \pm 0.033( \pm 5.1 \%)$ & 0.976 & 0.986 & 0.577 & 0.884 & \pm 0.045 \\
\hline 41 & IBKT & 46.96 & $0.688 \pm 0.033( \pm 4.8 \%)$ & 0.975 & 0.986 & 0.593 & 0.862 & \pm 0.042 \\
\hline 40 & IBKT & 52.48 & $0.675 \pm 0.033( \pm 4.9 \%)$ & 0.975 & 0.987 & 0.612 & 0.907 & \pm 0.045 \\
\hline 39 & IBKT & 58.01 & $0.759 \pm 0.033( \pm 4.4 \%)$ & 0.974 & 0.988 & 0.636 & 0.837 & \pm 0.037 \\
\hline 38 & IBKT & 63.54 & $0.761 \pm 0.033( \pm 4.4 \%)$ & 0.974 & 0.989 & 0.664 & 0.871 & \pm 0.038 \\
\hline 37 & IBKT & 69.06 & $0.772 \pm 0.033( \pm 4.3 \%)$ & 0.974 & 0.990 & 0.697 & 0.902 & \pm 0.039 \\
\hline 36 & IBKT & 74.59 & $0.824 \pm 0.033( \pm 4.0 \%)$ & 0.973 & 0.992 & 0.737 & 0.894 & \pm 0.036 \\
\hline 35 & IBKT & 80.11 & $0.866 \pm 0.033( \pm 3.8 \%)$ & 0.974 & 0.994 & 0.785 & 0.906 & \pm 0.035 \\
\hline 34 & IBKT & 85.63 & $0.892 \pm 0.035( \pm 3.9 \%)$ & 0.983 & 0.997 & 0.851 & 0.954 & \pm 0.037 \\
\hline 33 & DFC & 91.15 & $0.969 \pm 0.033( \pm 3.4 \%)$ & 0.996 & 0.999 & 0.931 & 0.961 & \pm 0.033 \\
\hline 32 & SFC & 96.68 & $0.997 \pm 0.033( \pm 3.3 \%)$ & 1.001 & 1.001 & 0.963 & 0.965 & \pm 0.032 \\
\hline 31 & SFC & 102.21 & $1.000 \pm 0.033( \pm 3.3 \%)$ & 1.002 & 1.002 & 0.959 & 0.959 & \pm 0.032 \\
\hline 30 & DFC & 107.73 & $0.963 \pm 0.033( \pm 3.5 \%)$ & 1.002 & 1.003 & 0.924 & 0.960 & \pm 0.033 \\
\hline 29 & $\mathrm{SFC}$ & 113.26 & $0.839 \pm 0.033( \pm 4.0 \%)$ & 1.002 & 1.004 & 0.850 & 1.012 & \pm 0.040 \\
\hline 28 & SFC & 118.78 & $0.742 \pm 0.033( \pm 4.5 \%)$ & 1.002 & 1.004 & 0.763 & 1.029 & \pm 0.046 \\
\hline 27 & DFC & 124.30 & $0.656 \pm 0.033( \pm 5.1 \%)$ & 1.001 & 1.004 & 0.653 & 0.995 & \pm 0.051 \\
\hline 26 & $\mathrm{SFC}$ & 129.83 & $0.469 \pm 0.033( \pm 7.1 \%)$ & 0.994 & 1.005 & 0.524 & 1.117 & \pm 0.080 \\
\hline 25 & RBKT & 135.35 & $0.424 \pm 0.033( \pm 7.9 \%)$ & 0.980 & 1.005 & 0.394 & 0.930 & \pm 0.073 \\
\hline 24 & RBKT & 140.88 & $0.263 \pm 0.033( \pm 12.7 \%)$ & 0.973 & 1.005 & 0.280 & 1.066 & \pm 0.135 \\
\hline 23 & RBKT & 146.40 & $0.203 \pm 0.033( \pm 16.4 \%)$ & 0.972 & 1.005 & 0.196 & 0.965 & \pm 0.159 \\
\hline 22 & RBKT & 151.92 & $0.081 \pm 0.033( \pm 41.1 \%)$ & 0.973 & 1.005 & 0.134 & & - \\
\hline 21 & RBKT & 157.45 & $0.117 \pm 0.033( \pm 28.5 \%)$ & 0.975 & 1.005 & 0.090 & & - \\
\hline 20 & RREF & 162.98 & $0.085 \pm 0.033( \pm 39.0 \%)$ & 0.984 & 1.005 & 0.056 & & - \\
\hline
\end{tabular}

$a_{E}$ normalized to a maximum value of 1.0 and then $C$ normalized such that the average $C / E$ in the core is 1.0.

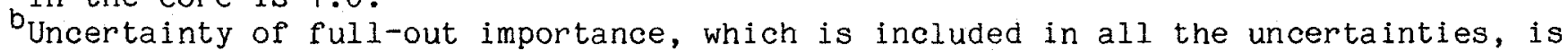
0.019 . 
TABLE 10.10 Clad PU-U + SS Column Worth Traverse, PCO-3

\begin{tabular}{|c|c|c|c|c|}
\hline \multirow{2}{*}{$\begin{array}{c}\text { Withdrawal } \\
(\mathrm{cm}) \\
\end{array}$} & \multicolumn{2}{|c|}{ Incremental Worth } & \multicolumn{2}{|c|}{ Cumulative Worth } \\
\hline & $E(\phi)$ & $\mathrm{C} / \mathrm{E}$ & $E(\phi)$ & $\mathrm{C} / \mathrm{E}$ \\
\hline 2.540 & $-0.10088 \pm 3.3 \%$ & $0.917 \pm 0.030$ & $-0.10088 \pm 3.3 \%$ & $0.917 \pm 0.030$ \\
\hline 12.699 & $-0.49258 \pm 0.6 \%$ & $0.996 \pm 0.006$ & $-0.59346 \pm 0.6 \%$ & $0.983 \pm 0.006$ \\
\hline 15.240 & $-0.14388 \pm 2.4 \%$ & $1.028 \pm 0.025$ & $-0.73734 \pm 0.5 \%$ & $0.992 \pm 0.005$ \\
\hline 17.780 & $-0.14565 \pm 2.4 \%$ & $1.078 \pm 0.026$ & $-0.88299 \pm 0.4 \%$ & $1.006 \pm 0.004$ \\
\hline 48.260 & $-1.83794 \pm 0.2 \%$ & $1.064 \pm 0.002$ & $-2.72093 \pm 0.1 \%$ & $1.045 \pm 0.001$ \\
\hline 65.412 & $-0.51668 \pm 0.6 \%$ & $1.013 \pm 0.006$ & $-3.23761 \pm 0.1 \%$ & $1.040 \pm 0.001$ \\
\hline
\end{tabular}


TABLE 10.11 $\mathrm{U}_{3} \mathrm{O}_{8}$ Column Worth Traverse, PCO-4

\begin{tabular}{|c|c|c|c|c|c|}
\hline \multirow{2}{*}{$\begin{array}{l}\text { Withdrawal } \\
(\mathrm{cm}) \\
\end{array}$} & \multicolumn{3}{|c|}{ Incremental Worth } & \multicolumn{2}{|c|}{ Cumulative Worth } \\
\hline & $E(\phi$ & & $\mathrm{C} / \mathrm{E}$ & $E(\phi)$ & $\mathrm{C} / \mathrm{E}$ \\
\hline 2.550 & $0.02710 \pm$ & $2.3 \%$ & $0.859 \pm 0.020$ & $0.02710 \pm 2.3 \%$ & $0.859 \pm 0.020$ \\
\hline 12.710 & $0.10003 \pm$ & $0.5 \%$ & $0.934 \pm 0.004$ & $0.12713 \pm 0.5 \%$ & $0.918 \pm 0.005$ \\
\hline 15.250 & $0.02474 \pm$ & $2.1 \%$ & $0.991 \pm 0.021$ & $0.15187 \pm 0.4 \%$ & $0.930 \pm 0.004$ \\
\hline 17.790 & $0.02408 \pm$ & $2.3 \%$ & $1.064 \pm 0.025$ & $0.17595 \pm 0.4 \%$ & $0.948 \pm 0.004$ \\
\hline 30.490 & $0.13263 \pm$ & $0.4 \%$ & $1.005 \pm 0.004$ & $0.30859 \pm 0.2 \%$ & $0.973 \pm 0.002$ \\
\hline
\end{tabular}


TABLE 10.12 Comparison of C/Es From PCO vs. RTO and ATO Experiments

\begin{tabular}{|c|c|c|c|}
\hline \multicolumn{2}{|c|}{ Plutonium Sample } & \multicolumn{2}{|c|}{ Depleted Uranium Sample } \\
\hline & $\mathrm{C} / \mathrm{E}$ & & $\mathrm{C} / \mathrm{E}$ \\
\hline \multicolumn{2}{|l|}{ PCO-1 } & \multicolumn{2}{|l|}{$\mathrm{PCO}-2$} \\
\hline $\mathrm{PCO}-1,2.54 \mathrm{~cm}$ & 1.20 & $\mathrm{PCO}-2,2.54 \mathrm{~cm}$ & 1.05 \\
\hline RTO, pos. \# 33 & 1.09 & RTO, pos. 非 33 & 1.00 \\
\hline PCO-1, core & 1.14 & PCO-2, core & 1.09 \\
\hline ATO-2, core & 1.05 & ATO-2, core & 1.02 \\
\hline \multirow{2}{*}{$\begin{array}{l}\text { PCO-1, ax. bkt. } \\
\text { ATO-2, ax. bkt. }\end{array}$} & 1.08 & \multirow{2}{*}{\multicolumn{2}{|c|}{$\mathrm{PCO}-4$}} \\
\hline & 1.19 & & \\
\hline \multicolumn{2}{|l|}{$\mathrm{PCO}-3$} & $\begin{array}{l}\mathrm{PCO}-4,2.55 \mathrm{~cm} \\
\mathrm{RTO}, \text { pos. \# } 39,41\end{array}$ & $\begin{array}{l}0.86 \\
0.86\end{array}$ \\
\hline $\mathrm{PCO}-3,2.54 \mathrm{~cm}$ & 0.92 & \multirow{2}{*}{\multicolumn{2}{|c|}{$\mathrm{PCO}-7$}} \\
\hline RTO, pos. \# 40 & 0.96 & & \\
\hline \multirow{2}{*}{$\begin{array}{l}\text { PCO-3, in. bkt. } \\
\text { ATO-1, in. bkt. }\end{array}$} & 0.99 & \multirow{2}{*}{$\begin{array}{l}\mathrm{PCO}-7,1.28 \mathrm{~cm} \\
\mathrm{RTO}, \text { pos. 非 } 31\end{array}$} & 1.09 \\
\hline & 1.04 & & 1.03 \\
\hline \multirow{2}{*}{$\begin{array}{l}\text { PCO-3, core } \\
\text { ATO-1, core }\end{array}$} & 1.07 & \multirow{2}{*}{\multicolumn{2}{|c|}{ Stainless Steel Sample }} \\
\hline & 1.00 & & \\
\hline \multirow{3}{*}{$\begin{array}{l}\text { PCO-3, ax. bkt. } \\
\text { ATO-1, ax. bkt. }\end{array}$} & 1.01 & \multirow[b]{3}{*}{$\begin{array}{l}\text { PCO-9, } 5.08 \mathrm{~cm} \\
\text { RTO, pos. \# } 31\end{array}$} & $\mathrm{C} / \mathrm{E}$ \\
\hline & 0.98 & & \\
\hline & & & $\begin{array}{l}1.50 \\
1.21\end{array}$ \\
\hline \multicolumn{4}{|l|}{$\mathrm{PCO}-5$} \\
\hline \multirow{2}{*}{$\begin{array}{l}\text { PCO-5, core } \\
\text { ATO-2, core }\end{array}$} & 1.07 & & \\
\hline & 1.05 & & \\
\hline \multicolumn{4}{|l|}{$\mathrm{PCO}-6$} \\
\hline $\mathrm{PCO}-6,1.02 \mathrm{~cm}$ & 1.15 & & \\
\hline RTO, pos. \# 31 & 1.11 & & \\
\hline
\end{tabular}


TABLE 10.13 Representative Core Region C/Es From PCO Experiments

\begin{tabular}{|c|c|c|}
\hline Traverse & Sample & $\underline{\mathrm{C} / \mathrm{E}}$ \\
\hline $\mathrm{PCO}-1$ & $50.8 \mathrm{~cm}-1$ ong $\mathrm{Clad} \mathrm{Pu-U}+15.2 \mathrm{~cm}$-long SS & 1.15 \\
\hline PCO-2 & $66.0 \mathrm{~cm}-1$ ong $\mathrm{U}_{3} \mathrm{O}_{8}$ & 1.08 \\
\hline $\mathrm{PCO}-3$ & $50.8 \mathrm{~cm}-10 n g \mathrm{Clad} \mathrm{Pu}-\mathrm{U}+15.2 \mathrm{~cm}-10 \mathrm{ng} \mathrm{SS}$ & 1.06 \\
\hline $\mathrm{PCO}-4$ & $101.6 \mathrm{~cm}-1$ ong $U_{3} 0$ & 1.01 \\
\hline PCO-5 & Last $66.0 \mathrm{~cm}$ of $101.6 \mathrm{~cm}-1$ ong Clad $\mathrm{Pu}-\mathrm{U}$ & 1.07 \\
\hline $\mathrm{PCO}-6$ & $101.6 \mathrm{~cm}-1$ ong $\mathrm{Clad} \mathrm{Pu}-\mathrm{U}$ & 1.16 \\
\hline PCO -7 & $101.6 \mathrm{~cm}-1$ ong $\mathrm{U} 0$ & 1.09 \\
\hline $\mathrm{PCO}-8-9$ & $101.6 \mathrm{~cm}^{-10 n g} \mathrm{Na}^{3}$ & $1.08^{\circ}$ \\
\hline PCO-9 & $101.6 \mathrm{~cm}$-long Void Cans (SS) & 1.36 \\
\hline PCO-10 & $101.6 \mathrm{~cm}$-1ong $\mathrm{Fe}_{2} \mathrm{O}_{3}$ & 1.35 \\
\hline
\end{tabular}

${ }^{a} \mathrm{C} / \mathrm{E}$ is 1.37 without corrections 
TABLE 10.14. Axial Expansion Oscillator Results

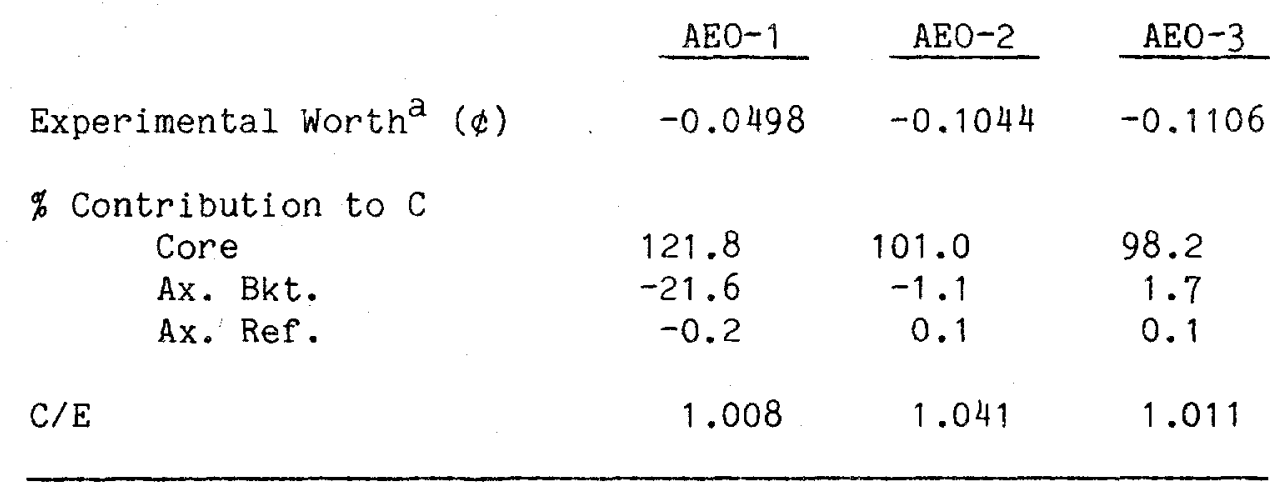

${ }^{a}$ Experimental uncertainty is about $3 \%$. 
TABLE 10.15 Bowing Oscillator C/E Adjustments

From YZ Model Calculations

Adjustment

Exact composition of $\mathrm{BO}$ in $\phi, \phi^{*}$ calculations Half-drawer $Y$-node spacing in $\phi, \phi^{*}$ calculations $S$ transport theory Interpolation Schemes ${ }^{\mathrm{a}}$ half-cell linear interpolation half-cell linear extrapolation half-cell piecewise constant

$-0.7 \%$

$-0.9 \%$

$+3.2 \%$

$-2.6 \%$

$-6.6 \%$

$-49.5 \%$

$a_{\text {Relative to }}$ full-cell linear interpolation 


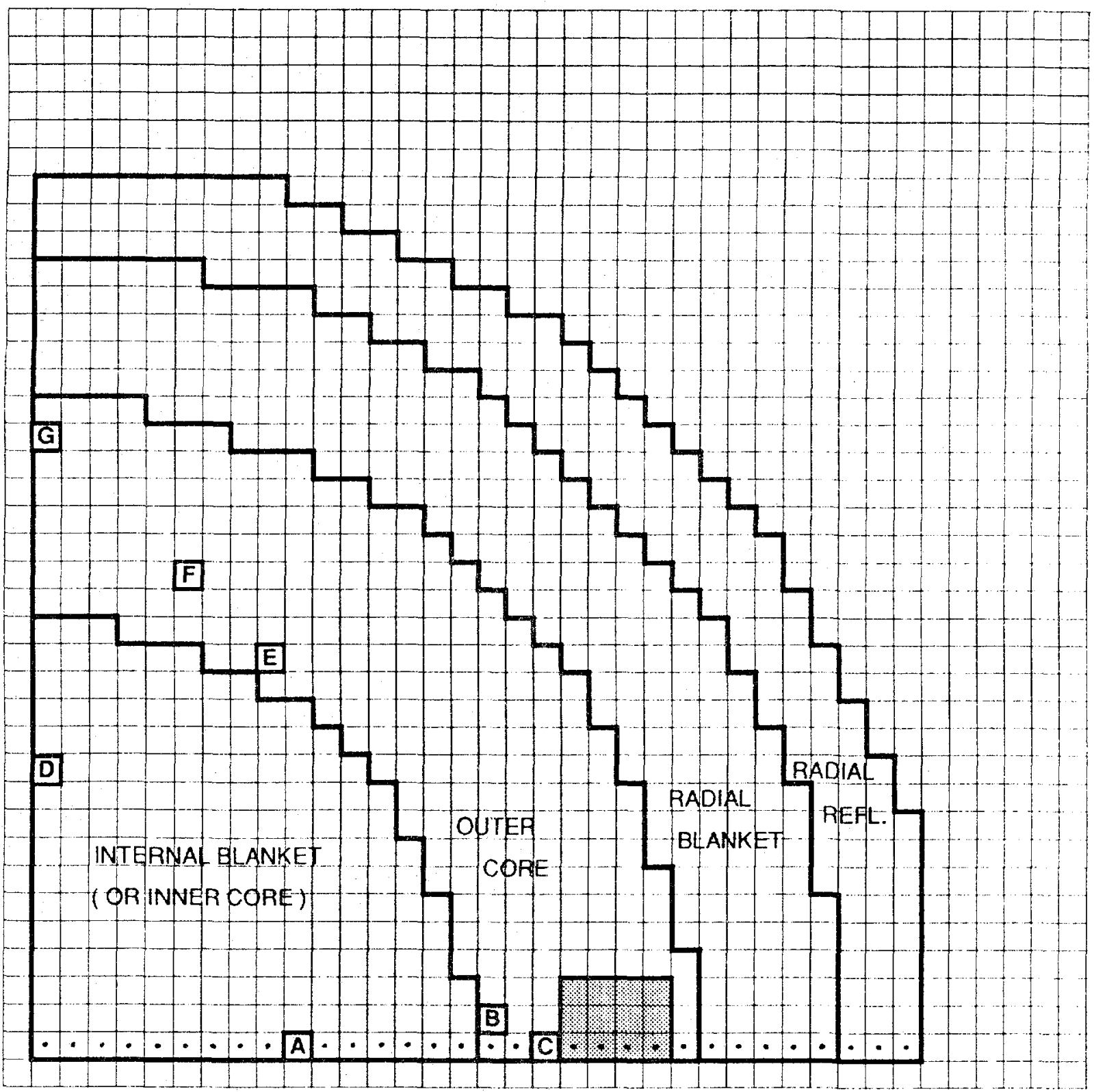
A $\quad \mathrm{PCO}-3, \mathrm{PCO}-4$
[E] ATO-2, AEO-2, PCO-5
B $\mathrm{PCO}-1, \mathrm{PCO}-2$
E AEO-3
C] PCO-6 -- PCO-10
G ATO-3,BO
[ ATO-1, AEO-1
PLATE SHIFTING ZONE
. RTO-1, RTO-2

Fig. 10.1 Locations of Plate Shifting Zone and Oscillators in ZPPR-17A 
PU-30, Radial Traverse

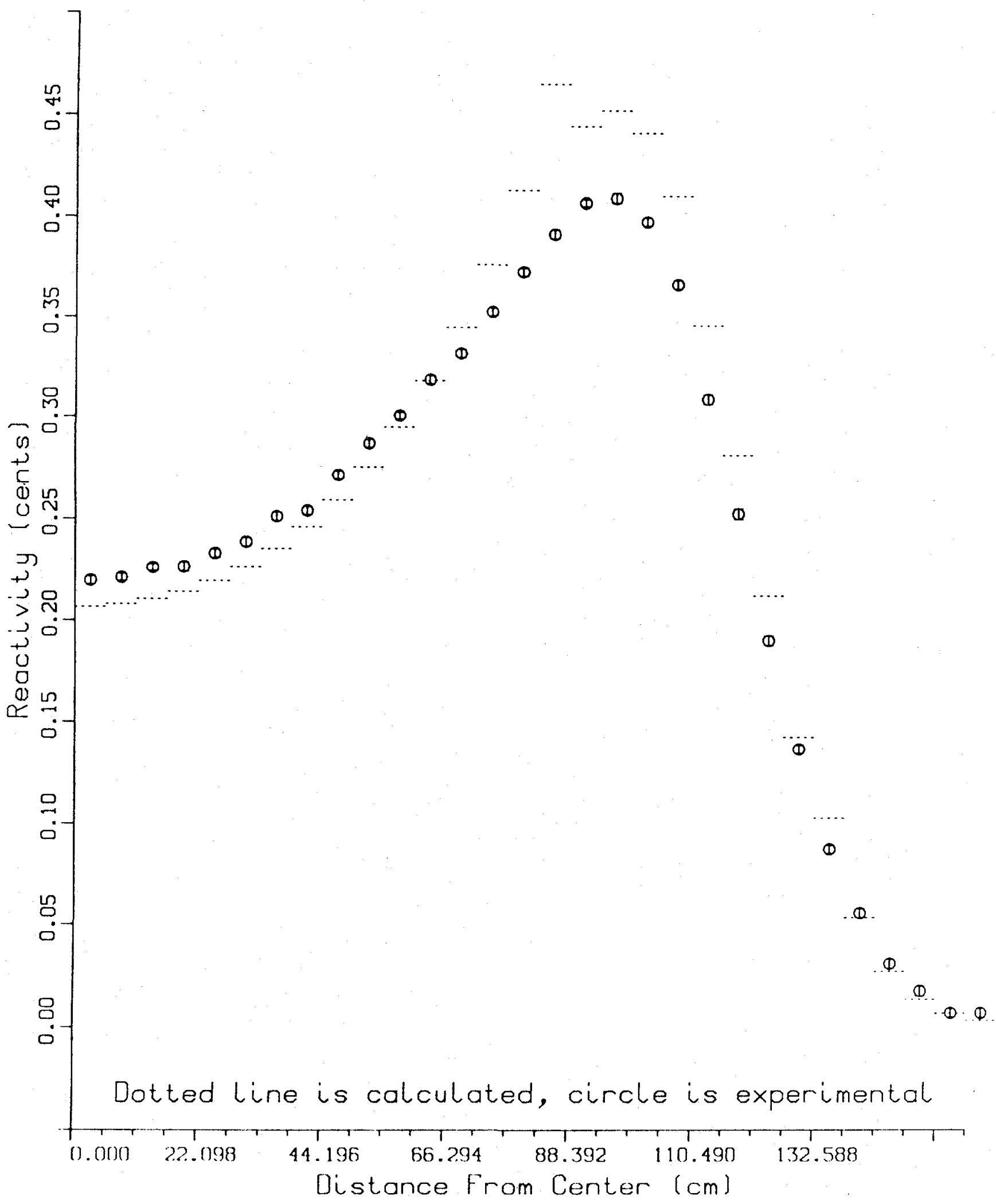

Fig. 10.2 Radial Traverse for PU-30 Sample in ZPPR-17A. 
DU-6, Radial Iraverse

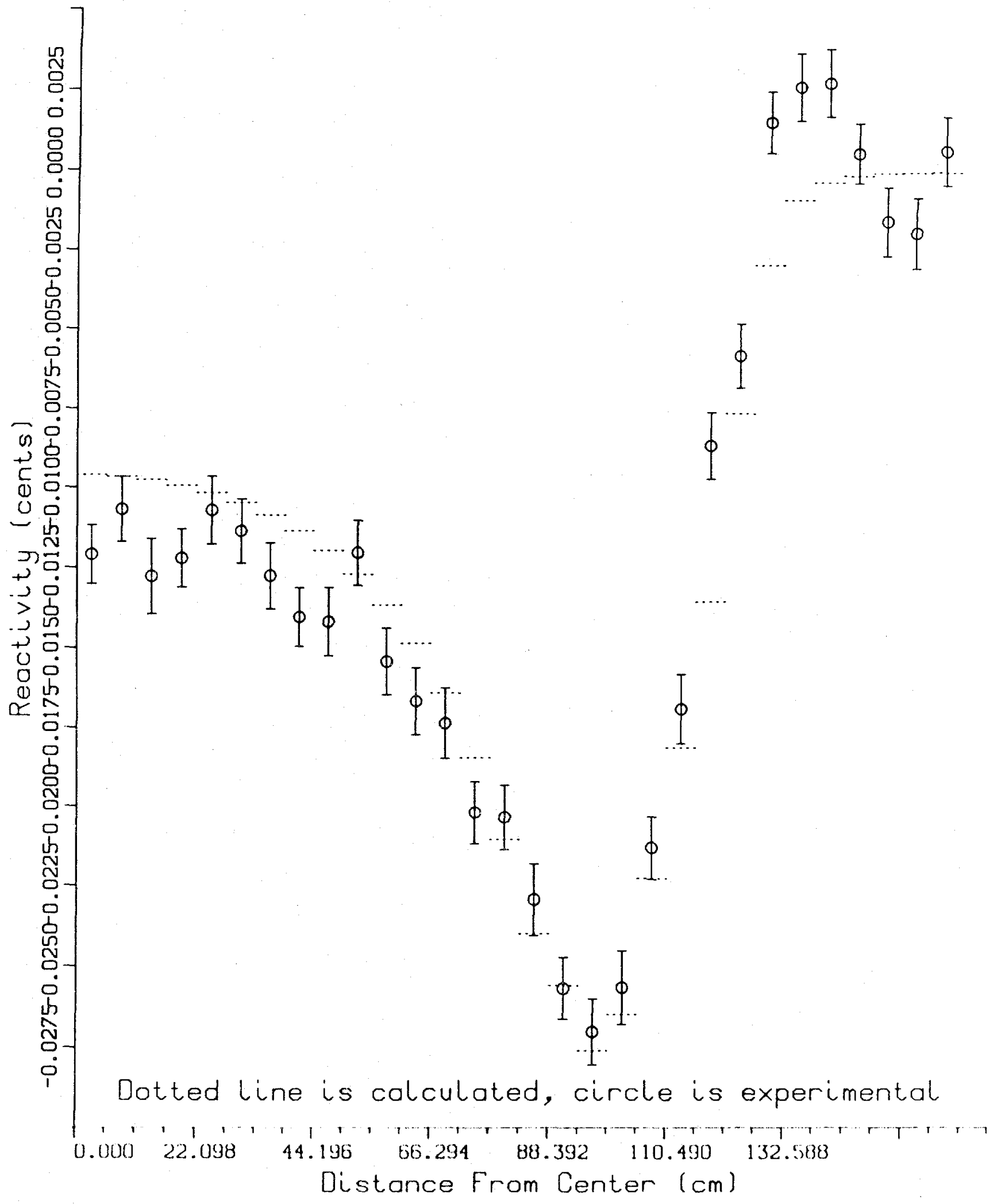

Fig. 10.3 Radial Traverse for DU-6 Sample in ZPPR-17A. 
PU-30, First Rxial Traverse (159-49)

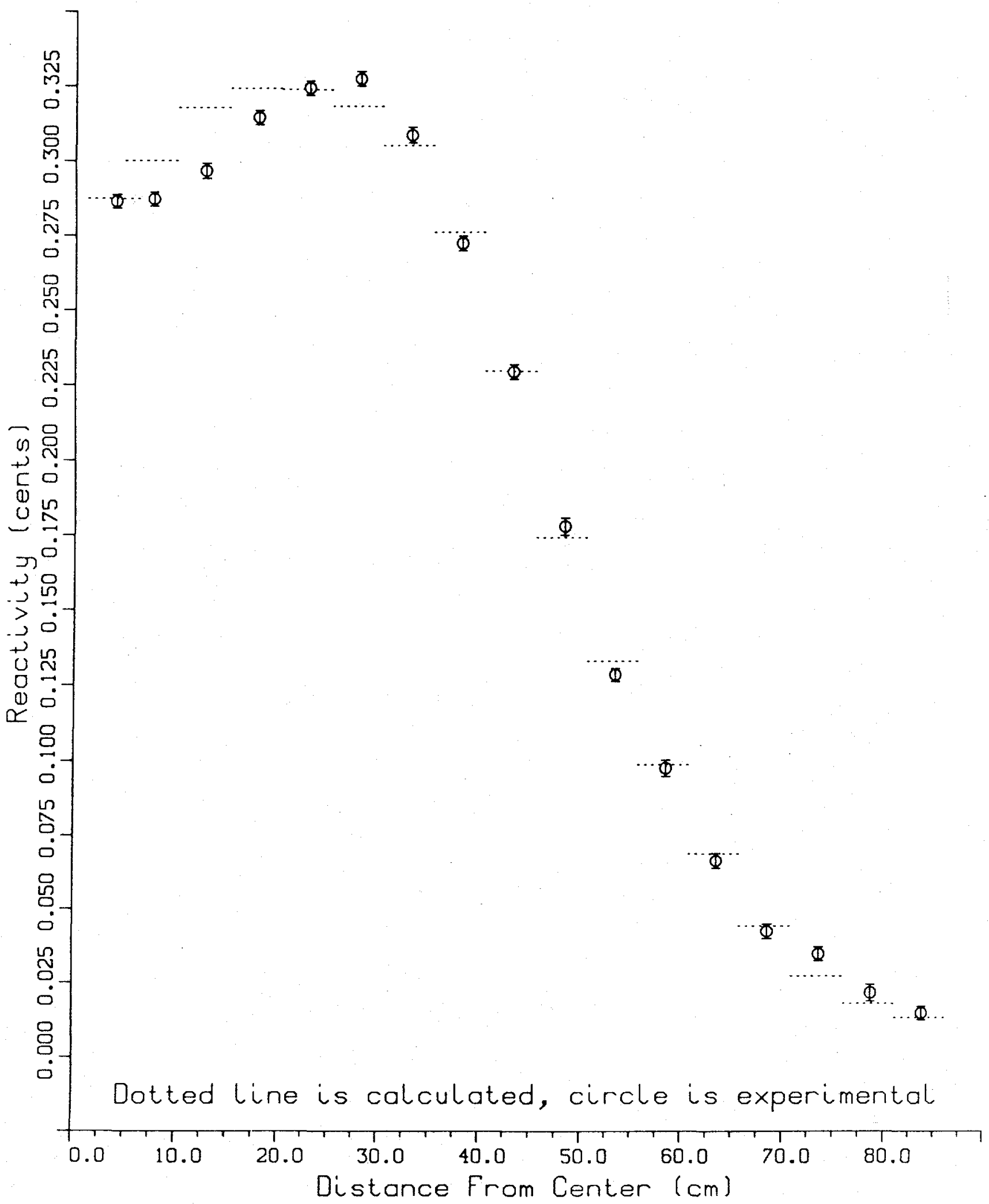

Fig. 10.4 First Axial Traverse (ATO-1) for PU-30 Sample in ZPPR-17A. 


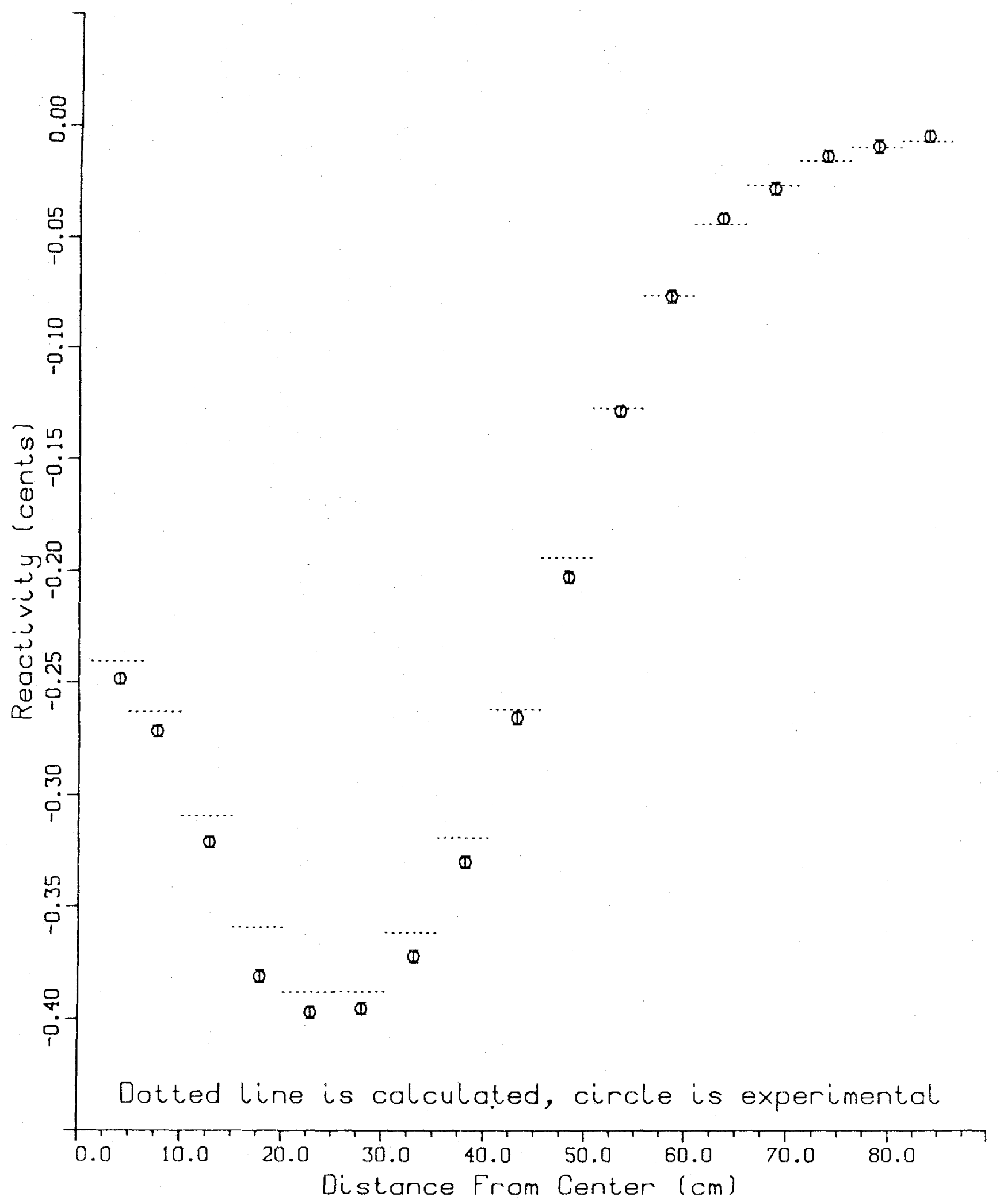

Fig. 10.5 First Axial Traverse (ATO-1) for B-1 Sample in ZPPR-17A. 
U-6, First Axial Traverse (159-49)

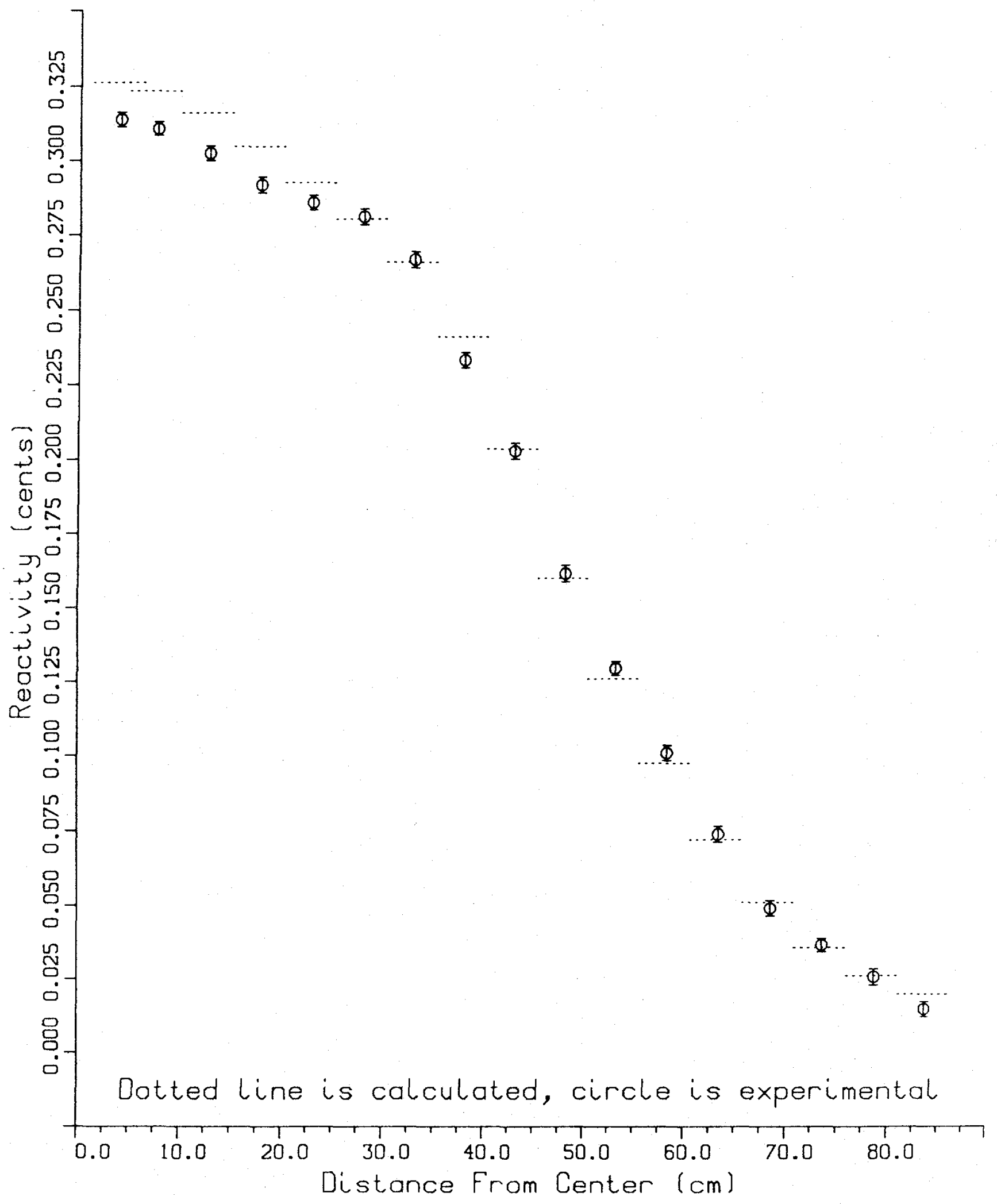

Fig. 10.6 First Axial Traverse (ATO-1) for U-6 Sample in ZPPR-17A. 
Neutron Importance Radial Traverse

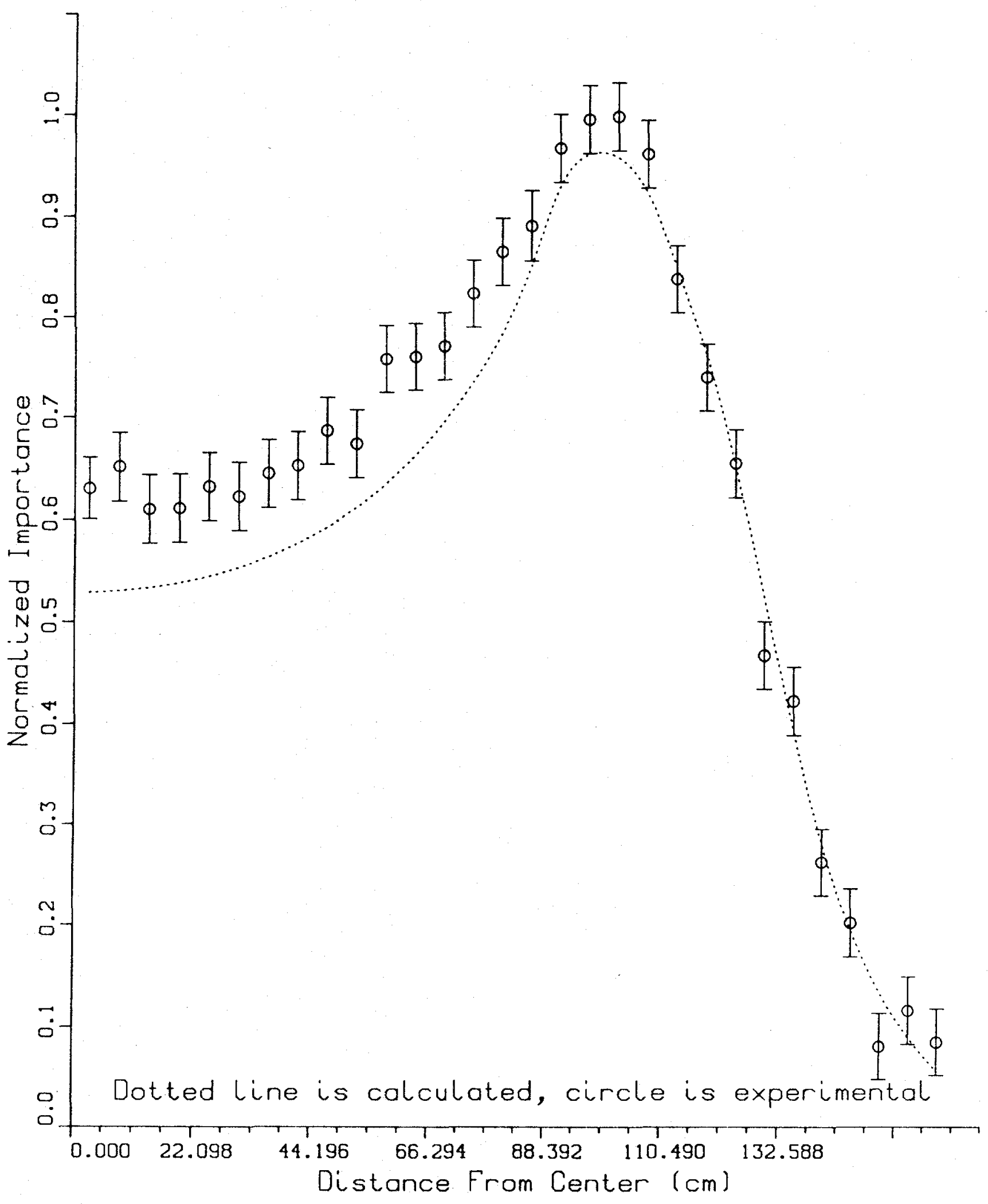

Fig. 10.7 Neutron Importance Radial Traverse in ZPPR-17A. 
REFERENCES

1. P. J. Collins and S. B. Brumbach, eds., "ZPPR Progress Report: April 1987 through July 1987, "ANL-ZPR-476, (1987).

2. S. B. Brumbach and P. J. Collins, eds., "ZPPR Progress Report: August 1987 through October 1987," ANL-ZPR-480 (1987).

3. P. J. Collins and S. B. Brumbach, eds., "ZPPR Progress Report: November 1987 through January 1988," ANL-ZPR-481 (1988).

4. S. B. Brumbach and P. J. Collins, eds., "ZPPR Progress Report: February 1988 through April 1988," ANL-ZPR-482 (1988).

5. P. J. Collins and S. B. Brumbach, eds., "ZPPR Progress Report: May 1988 through August 1988," ANL-ZPR-485 (1988).

6. "The JUPITER Program: ANL Analys is of ZPPR-9," Report prepared for the First Analysis Meeting on JUPITER Program, September 11 and 12, 1980.

7. "The JUPITER Program: Analysis of ZPPR-10," Report prepared for the Second Analysis Meeting on JUPITER Program, October 19-26, 1981.

8. "The JUPITER-II Program: ANL Analysis of ZPPR-13A and ZPPR-13B," Report prepared for the JUPITER Analysis Meeting, Sept. 11-14, 1984.

9. S. B. Brumbach and P. J. Collins, eds., "ZPPR Progress Report: January 1987 through March 1987," ANL-ZPR-473 (1987).

10. H. Unesaki, "Analysis of ZPPR-17 Gamma Heating Experiments," ANL-ZPR-483 (1988).

11. R. E. Alcouffe, F. W. Brinkley, D. R. Marr and R. D. O'Dell, "User's Guide for TWODANT: A Two-Dimensional Diffusion Accelerated Neutral Particle Discrete Ordinates Transport Code," LA-10049-M, Los Alamos National Laboratory, Los Alamos, NM (1984).

12. M. Bando et a.,, "Three-Dimensional Transport Calculation Method for Eigenvalue Problems Using Diffusion Synthetic Acceleration,". J. Nucl. Sci. Tech., Vol. 22, No. 10, pp 814-850, October 1985.

13. R. D. Lawrence, "Three-Dimensional Nodal Diffusion and Nodal Transport Methods for the Analysis of Fast-Reactor Critical Experiments," Proc. Topical Meeting on Reactor Physics and Shielding, Chicago, IL, $17-19$ September 1984, p. 814.

14. C. A. Atkinson and P. J. Colliins, "The Performance of ENDF/B-V.2 Nuclear Data for Fast Reactor Calculations," Proc. of the International Conference on Reactor Physics and Mathematics and Computation, Paris, France, 27-30 April 1987. 
15. S. B. Brumbach and D. W. Maddison, "Reaction Rate Calibration Techniques at ZPPR for ${ }^{239} \mathrm{Pu}$ Fission, ${ }^{235} \mathrm{U}$ Fission, ${ }^{238} \mathrm{U}$ Fission, and ${ }^{238} \mathrm{U}$ Capture," ANL-82-38 (1982).

16. W. P. Poenitz, D. W. Maddison, J. M. Gasidlo, S. G. Carpenter and R. J. Armani, $"{ }^{235} U(n, f),{ }^{238} U(n, \gamma),{ }^{238} U(n, f)$ and ${ }^{239} P u(n, f)$ Reaction Rate Measurement Calibrations at ZPPR," ANL-87-5 (1987).

17. K. S. Smith, "The Effects of Intracell Adjoint Flux Heterogeneity on First-Order Perturbation Reactivity Calculations," Nucl. Sci. Eng., 81, p. 451 (1982).

18. R. W. Schaefer, "Calculations of Bowing, Expansion and Small-Sample Worth Experiments in ZPPR-17A," ANL-ZPR-488 (1988).

19. R. W. Schaefer, "Critical Experiment Tests of Bowing and Expansion Reactivity Calculations for LMRs," Proc. of the 1988 International Reactor Physics Conference, September 18-22, 1988, Jackson Hole, WY, to be published.

20. P. J. Collins and R. G. Palmer, "Calculated Size Effects for Reactivity Perturbation Samples in ZPPR," ANL-7910, p. 247, Argonne National Laboratory (1971).

21. P. J.Finck, "A Technique for Computing Bowing Reactivity Feedback in LMFBRs," Trans. Am. Nucl. Soc. 55, p. 581 (1987). 